NISTIR 90-3935

\title{
DEVELOPMENT OF STANDARDS FOR SUPERCONDUCTORS
}

\section{Final Report \\ January 1988-December 1989}

L.F. Goodrich, R.B. Goldfarb, S.L. Bray

Electromagnetic Technology Division

Center for Electronics and Electrical Engineering

National Engineering Laboratory

National Institute of Standards and Technology

Boulder, Colorado 80303-3328

\section{JANUARY 1990}

$Q C$

100

.456

N0.90-3935

1990 



\section{DEVELOPMENT OF STANDARDS FOR SUPERCONDUCTORS}

\section{Final Report \\ January 1988-December 1989}

L.F. Goodrich, R.B. Goldfarb, S.L. Bray

Electromagnetic Technology Division

Center for Electronics and Electrical Engineering

National Engineering Laboratory

National Institute of Standards and Technology

Boulder, Colorado 80303-3328

\section{JANUARY 1990}
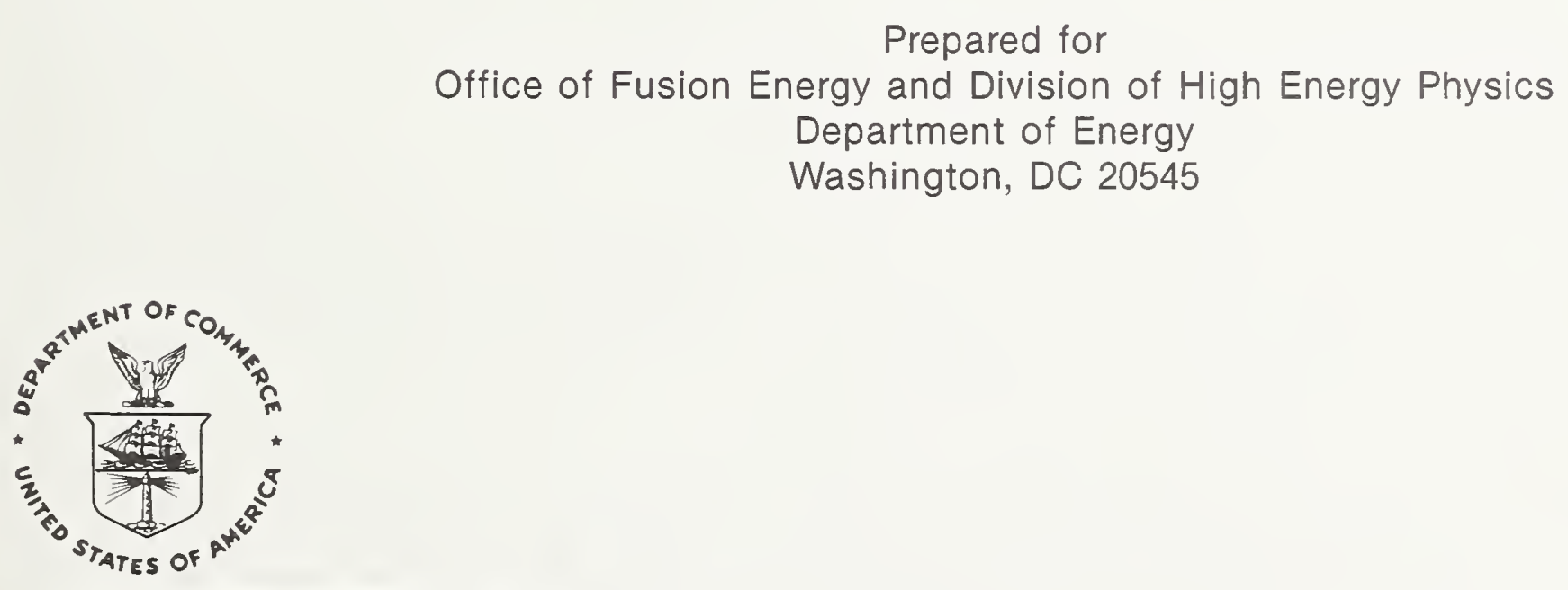

DEPARTMENT OF COMMERCE, Robert A. Mosbacher, Secretary NATIONAL INSTITUTE OF STANDARDS

AND TECHNOLOGY, Raymond G. Kammer, Acting Director 

The National Institute of Standards and Technology (formerly the National Bureau of Standards) program to develop standards for conventional superconductors has been one of our most successful and visible projects in support of DoE superconductor applications. Appendix A is a list of publications from this research program. We have been successful in standardizing measurement techniques in practical superconductivity. This has resulted in savings of time and money as well as better communication among researchers, producers, and users of superconductors. The work described here is a further effort in this area. We are shifting our emphasis to large conductor standards and topics such as ac losses which have become of increasing concern with the development of fine filament conductors. Small conductor work continues as well with emphasis on round robin methods to measure the degree to which our suggested techniques have increased measurement uniformity and accuracy. 

FOREWORD

ABSTRACT

I. CRITICAL CURRENT

II. AC LOSSES

III. ADDITIONAL ACTIVITIES

IV. ACKNOWLEDGEMENTS

V. REFERENCES

Appendix A - PUbliCATIONS OF RESEARCH PROGRAM . . . . . . . . . . . 25

Appendix B - INTEGRITY TESTS FOR HIGH-T $\mathrm{C}$ AND CONVENTIONAL CRITICALCURRENT MEASUREMENTS SYSTEMS

L. F. Goodrich and S. L. Bray . . . . . . . 31

Appendix C - BATTERY-POWERED CURRENT SUPPLY FOR SUPERCONDUCTOR MEASUREMENTS

S. L. Bray, L. F. Goodrich, and W. P. Dube

Appendix D - NB 3 SN CRITICAL-CURRENT MEASUREMENTS USING TUBULAR FIBERGLASS - EPOXY MANDRELS

L. F. Goodrich, S. L. Bray, and T. C. Stauffer.

Appendix E - CRITICAL CURRENT MEASUREMENTS OF NB 3 SN SUPERCONDUCTORS: NBS CONTRIBUTION TO THE VAMAS INTERLABORATORY COMPARISON

L. F. Goodrich and S. L. Bray . . . . . . . 53

Appendix F - THERMAL CONTRACTION OF FIBERGLASS-EPOXY SAMPLE MANDRELS AND ITS EFFECT ON CRITICAL-CURRENT MEASUREMENTS

L. F. Goodrich, S. L. Bray, and T. C. Stauffer. 65 
Appendix G - THERMAL CONTRACTION OF FIBERGLASS-EPOXY SAMPLE

HOLDERS USED FOR NB 3 SN CRITICAL-CURRENT MEASUREMENTS

L. F. Goodrich, S. L. Bray, and T. C. Stauffer . 69

Appendix $\mathrm{H}$ - VAMAS INTERCOMPARISON OF CRITICAL CURRENT MEASUREMENT IN $\mathrm{NB}_{3} \mathrm{SN}$ WIRES

K. Tachikawa, K. Itoh, H. Wada, D. Gould, H. Jones

C. R. Walters, L. F. Goodrich, J. W. Ekin, and

S. L. Bray . . . . . . . . . . . . 79

Appendix I - PROPOSED STUDY ON THE EFFECT OF SAMPLE BONDING TECHNIQUES ON THE MEASURED CRITICAL CURRENT OF NB 3 SN SUPERCONDUCTORS

L. F. Goodrich and S. L. Bray . . . . . . . 87

Appendix J - CURRENT RIPPLE EFFECT ON SUPERCONDUCTIVE D.C. CRITICAL CURRENT MEASUREMENTS

L. F. Goodrich and S. L. Bray . . . . . . . . 99

Appendix $\mathrm{K}$ - CALORIMETRIC MEASUREMENTS OF AC LOSS IN NB 3 SN SUPERCONDUCTORS

M. Takayasu, C. Y. Gung, M. M. Steeves, B. Oliver,

D. Reisner, and M. O. Hoenig . . . . . . 107

Appendix L - GUIDELINE FOR NB 3 SN CRITICAL CURRENT MEASUREMENTS USING FIBERGLASS-EPOXY COMPOSITE SAMPLE MANDRELS (SUMMARY)

L. F. Goodrich ........... 115

Appendix M - REPORT ON SAMPLE MOUNTING

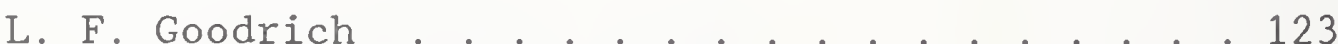

Appendix $\mathrm{N}$ - GUIDELINE FOR $\mathrm{NB}_{3} \mathrm{SN}$ CRITICAL CURRENT MEASUREMENTS USING FIBERGLASS - EPOXY COMPOSITE SAMPLE MANDRELS

L. F. Goodrich . . . . . . . . . . . . . 129 


\section{F. Goodrich, R. B. Goldfarb, and S. L. Bray Electromagnetic Technology Division Center for Electronics and Electrical Engineering National Engineering Laboratory National Institute of Standards and Technology Boulder, Colorado 80303}

A cooperative program with the Department of Energy, the National Institute of Standards and Technology, other national laboratories, and private industry is in progress to develop standard measurement practices for use in large-scale applications of superconductivity. This report describes research for the period January 1988 through December 1989. It contains the results of critical current studies on a large conductor Reference Material (RM), the effect of power-supply current ripple, the measurements of large conductors, and an interlaboratory comparison (round robin) of $\mathrm{Nb}_{3} \mathrm{Sn}$ wires. Short-range variations in magnetic hysteresis loss in multifilamentary $\mathrm{Nb}_{3} \mathrm{Sn}$ were studied. The results of participation in several interlaboratory comparisons are described.

Key words: ac losses; circuit diagrams; critical current; current ripple; hysteresis; magnetization; niobium-tin; niobium-titanium; power supply; round robin; standards; superconductors; susceptibility. 



\section{CRITICAL CURRENT}

\section{A. Large Conductor Reference Material}

Critical current measurement requires complex techniques and apparatus. The quality of the measurement is strongly dependent on the integrity of the overall system. There are numerous measurement variables that, if not properly addressed, can cause erroneous results. The NbTi superconducting Wire Standard Reference Material (SRM 1457) [1] was developed to provide a holistic method for testing $I_{c}$ measurement systems. The SRM has been productively utilized from both a scientific and commercial standpoint; however, its usefulness is limited by its maximum current capacity. At a magnetic field of $6 \mathrm{~T}$, the $\mathrm{I}_{\mathrm{C}}$ of the SRM is only $125 \mathrm{~A}$, but it has been used to test 25,000 A $I_{c}$ measurement systems [2]. This situation suggested the need for a well characterized superconductor having a significantly higher $I_{c}$.

It was decided that a RM (reference material) rather than an SRM was the best solution to the need for higher current capacity in a well-characterized conductor. The characterization of a RM is not as stringent as a SRM and, thus, its properties are not certified at the level of a SRM. There are two basic reasons, one practical and one technical, why this is appropriate for the large conductor. Technically, there are measurement problems unique to large conductors that, at present, limit our confidence in measurement accuracy. A matter of practicality is the limited distribution anticipated for the large conductor RM as compared to the SRM. This limited distribution diminishes the benefit of the additional time and difficulty required to develop a SRM.

A preliminary screening of candidate conductors for the RM resulted in the selection of a NbTi rectangular monolith. A U.S. wire manufacturer has agreed to supply NIST with the required length of this conductor $(\sim 500 \mathrm{~m})$ at no charge. The cross-sectional dimensions of the conductor are $1.8 \mathrm{~mm} \times 3.6$ $\mathrm{mm}$ and its $I_{c}$ at $6 \mathrm{~T}$ is approximately $2700 \mathrm{~A}$, or about 22 times that of the SRM. A $12 \mathrm{~T}, 12.7 \mathrm{~cm}$-diameter (5") bore magnet, that is well suited to large conductor $I_{c}$ measurements, has been acquired, tested, and calibrated. A sample test fixture that incorporates 3000 A vapor cooled current leads and a magnet-to-test-fixture coupling that is intended to support large Lorentz forces has been designed, constructed, and tested. The test fixture will accommodate multiturn coiled samples having diameters up to $11.4 \mathrm{~cm}$. Calibrations have been made on current, voltage, magnetic field, and temperature.

The wire was received as a continuous length from which 130 sample units were made. A systematic sampling pattern was used to select 30 units from these 130. The remaining 100 units will be available for sale. The 30 sample units are used to characterize all units. It was decided that about 10 critical current specimens would be chosen from these 30 units. Each sample unit, or spool, has approximately 3 meters of wire, which is more than enough length for an $I_{c}$ specimen. The extra length on these units and the remainder of the 30 units are to be used for photographs, other measurements, and contingencies. The filament twist pitch, copper-to-superconductor ratio, 
average filament diameter, and superconductor area are examples of other measurements now being made. An anticipated contingency is the possibility of testing a suspect measurement with an adjacent specimen.

The critical current or the electric field (or resistivity) versus current is being characterized over a range of variables. The approximate range of electric fields is from 0.02 to $0.5 \mu \mathrm{V} / \mathrm{cm}$. The approximate range of resistivity is from $10^{-12}$ to $10^{-11} \Omega \cdot \mathrm{cm}$ (except at the higher magnetic fields). The range of applied magnetic fields is 5 to $9 \mathrm{~T}$. The sample temperature ranges from 4.02 to $4.22 \mathrm{~K}$. The highest current is about $3850 \mathrm{~A}$ and the lowest is about $670 \mathrm{~A}$. Measurements are made with both current directions with the applied magnetic field parallel to the wide face of the conductor.

At present data acquisition on the test samples is nearly completed and statistical modeling is being developed. The target date for the RM to be available is in 1990. Several additional related publications are planned. paper on a simple test (integrity test) of the critical-current measurement system is included as Appendix B to this report. A final paper on our high current supply is included as Appendix $C$.

\section{B. Self-Field Effect}

A variable that can significantly affect short sample $I_{c}$ measurements in high current conductors is the magnetic field introduced by the current carried in the test sample and its current leads. This "self field" is superimposed on the applied background field and can either increase or decrease the magnitude of the total magnetic field, depending on its orientation with respect to the background field. The effect of this variable on the measured $I_{C}$ depends on the magnitude and the spatial geometry of the sample current including the current return path. For aspected conductors (those that have unequal cross-sectional dimensions, a rectangular conductor for instance), the $I_{c}$ depends on the orientation of the magnetic field in the plane of its cross-sectional axes. Consequently, the measured $I_{c}$ will fall in a range of possible values depending on the field orientation. The purpose of this study is to measure that range and to determine techniques that can reduce the measurement uncertainty introduced by this effect.

The NbTi monolith that was selected for the large conductor reference material was also used in this study. The knowledge obtained in this study regarding its behavior with respect to the self-field effect should be beneficial in characterizing it for use as a RM. A sample holder was designed and constructed that allowed measurement of the self-field effect for several different sample current geometries. The initial self-field measurements have been completed and the resulting data have been analyzed. This has proven to be a formidable task because of the complexity of accurately determining the nature of the magnetic field perturbations caused by the self-field of the various sample and current-lead geometries. The agreement between the measured values and the calculated values was very good; for example, a $9.1 \%$ change in the measured $I_{C}$ was predicted to be 10.6\%. A publication of these 
results and the results of other self-field experiments is planned in the next year.

There is also concern about variation in magnetization among wires that are processed in different ways. This problem presents another possible application for our self-field calculations; to determine the actual shape of $\mathrm{I}_{\mathrm{c}}(\mathrm{H})$ from 0 to $8 \mathrm{~T}$ without the self-field effect. These data are relevant to the magnetization of a conductor. At this time it is not known how changes in $I_{c}$ in the region from 5 to $8 \mathrm{~T}$ will reflect changes in $\mathrm{I}_{c}$ at low magnetic fields. Thus, variations in $I_{C}(H)$ need to be understood and controlled in order to control variation in magnetization. We have demonstrated our capability of measuring $\mathrm{I}_{c}(\mathrm{H})$ from 0 to $8 \mathrm{~T}$ on an Superconducting Super Collider prototype conductor (see Section $\mathrm{I}-\mathrm{E}$ ). The $\mathrm{I}_{\mathrm{C}}$ was about $1700 \mathrm{~A}$ in zero applied magnetic field. A systematic study of the correlation between variation at low fields and variation in the region of 5 to $8 \mathrm{~T}$ may allow estimates of low field variation using the variation measured in the region of 5 to $8 \mathrm{~T}$, which can be determined during more routine measurements.

\section{C. $\quad \mathrm{Nb}_{3} \mathrm{Sn}$ Round Robins}

A significant research activity has been the continuing participation in the VAMAS (Versailles Agreement on Advanced Materials and Standards) and the US-Japan interlaboratory comparative measurements, or round robins, on the critical current $\left(\mathrm{I}_{\mathrm{C}}\right.$ ) of $\mathrm{Nb}_{3} \mathrm{Sn}$. Twenty-four laboratories from Europe, Japan, and the United States participated in the round robin. The purpose of this international collaborative effort is to determine the sources of variation in the measured $\mathrm{I}_{c}$ of $\mathrm{Nb}_{3} \mathrm{Sn}$ superconductors and, ultimately, to arrive at an accepted measurement technique that is general enough to be practical and yet specific enough to yield consistent and accurate results at different laboratories. In response to discrepancies in the results of these comparative measurements, the NIST study was expanded to address specific measurement variables (thickness of G-10 sample holder and thermal contraction) that were thought to be partially responsible for these discrepancies. Four papers relating to the expanded NIST study are included as Appendices D, E, F, and G to this report. The first paper on the joint results of the VAMAS round robin is included as Appendix $H$. The second joint results paper was published in the last year [3]. The NIST work has identified and suggested a solution to a problem that was a significant source of the measurement discrepancies.

Preliminary comparisons of the data showed variations about the average measured $I_{C}$ of $\pm 50 \%$ at a magnetic field of $12 \mathrm{~T}$. The largest variations in the measured $I_{C}$ were thought to be the result of sample damage incurred during handling. However, even with this outlying data omitted, the variation for some of the samples is still $\pm 25 \%$. The NIST systematic study of the effect of measurement holder (tubular sample holder made from G-10 fiberglass-epoxy composite) geometry revealed that a seemingly small change in geometry can result in a $40 \%$ change in the measured $I_{c}$ at a magnetic field of $12 \mathrm{~T}$. Specifically, the radial thermal contraction of the measurement mandrel depends on its wall thickness and, thus, so do the conductor prestrain 
(at $4 \mathrm{~K}$ ) and, ultimately, the measured critical current. This study revealed that axial sample strain was a principal variable in the measurement.

Three different conductors were used in these measurements; one was manufactured in Europe, one in Japan, and one in the United States. These conductors varied in overall diameter, filament diameter, manufacturing process (internal tin or bronze process), and copper to superconductor ratio. Owing to these variations, some samples were more sensitive to certain measurement variables than others. Although such sensitivities probably resulted in a greater overall data scatter, they were beneficial in the sense that they emphasized the importance of the specific measurement variables.

The large discrepancies in the measurements suggest that there is more to be learned about short-sample $\mathrm{Nb}_{3} \mathrm{Sn} \mathrm{I}_{c}$ measurements. Several topics that require further investigation are sample holder materials, reaction variables, bonding techniques, and sample homogeneity. In the category of sample holder materials, two measurement variables are of particular concern: One is the reaction holder and the other is the measurement holder; both can cause large variations in the measured $I_{c}$. In the case of the reaction holder, usually an oxidized stainless steel tube, the sample can be damaged when it is removed from the holder following the reaction process, if diffusion bonding between the sample and holder has occurred. This problem might be alleviated by either an improved oxidization process for the stainless steel holders or by the use of a different reaction holder material that is less susceptible to bonding. In the case of the measurement holder, differential thermal contraction between the sample and holder can result in various states of sample strain depending on the sample holder material and its geometry and the bonding method. To address this problem, measurement holder materials and geometries that do not result in adverse mechanical interaction between the sample and holder need to be studied further.

The existing American Society for Testing and Materials (ASTM) "Standard Test Method for D-C Critical Current of Composite Superconductors" (B714-82) addresses the problem of differential thermal contraction between the sample and holder. The ASTM standard states that "The tensile strain, induced by the differential thermal contraction of the specimen and holder, shall not exceed 0.05 for $\mathrm{Nb}_{3} \mathrm{Sn} . "$ It was assumed that the sample holder materials supplied to a subset of the round robin participants, National Electrical Manufacturers' Association (NEMA) G-10 and G-11 tubes, met this specification. However, the effect of the holders wall thickness on its circumferential thermal contraction was not considered and, consequently, the ASTM maximum strain specification was exceeded in some measurements. In retrospect, the measurement discrepancies introduced by the use of sample holders having different wall thicknesses and, thus, different thermal contractions might have been anticipated and avoided.

The purpose of the round robin measurements is to document and better understand the sources of inconsistencies in the measured critical current of $\mathrm{Nb}_{3} \mathrm{Sn}$ conductors. This understanding is ultimately necessary for determining a consistent and practical measurement method. In theory, a method that would give consistent results is easily achieved by imposing extensive constraints on all aspects of the measurement; however, this approach is impractical 
considering the variety of $I_{c}$ measurement systems that are presently in use. A practical measurement method should impose the smallest number of constraints but yield acceptable results.

More challenging than the issue of consistent measurements is that of accurate measurements. The basic problem that these round robin tests reveal is that the correct and/or best method for critical-current measurements of $\mathrm{Nb}_{3} \mathrm{Sn}$ is presently unclear. This difficulty is a result of the strain sensitivity of $\mathrm{Nb}_{3} \mathrm{Sn}$ conductors. The question is, at what strain level should the sample's $I_{C}$ be measured and how can this selected strain level be consistently reproduced (with practicality in mind) at different laboratories?

Theoretically, this problem could be solved by simply requiring that all laboratories measure the $\mathrm{I}_{\mathrm{C}}$ of $\mathrm{Nb}_{3} \mathrm{Sn}$ as a function of tensile strain; however, there are several reasons why this is not a practical solution. The measurement of $I_{C}$ as a function of strain requires specialized equipment that is only available in a few laboratories. Also, among the laboratories that do have this measurement capability, there are often considerable discrepancies. These may be partially because that $I_{C}$-strain measurements are usually made using straight, rather than coiled, samples. The straight samples are normally shorter than coiled samples; consequently, the increased current transfer voltage and decreased voltage tap separation associated with the shorter samples can reduce measurement accuracy significantly. In general, the $I_{c}$-strain measurement is a complex one that introduces a number of new measurement variables and, thus, it is unlikely that this approach would enhance measurement consistency.

NIST was asked to prepare a document on a "Proposed Study of the Effect of Sample Bonding Techniques on the Measured Critical Current of $\mathrm{Nb}_{3} \mathrm{Sn}$ Superconductors" (Appendix I). This is a detailed test plan to determine if grease can be used as a sample bonding material instead of solder. This document was published in the Proceedings of the $6^{\text {th }} \mathrm{Japan-US}$ Workshop on High-Field Superconducting Materials and Standards Procedures for High-Field Superconducting Materials Testing.

\section{Large Conductor $I_{c}$ Test Method}

The work that has already been completed in the area of large-conductor measurements and the planning for future studies has resulted in some special concerns regarding the measurement of large-conductor critical currents. These concerns fall into three related categories: increased electromagnetic forces (Lorentz force), power supply effects, and large-conductor characteristics.

The high current levels required for large-conductor measurements result in increased Lorentz forces on the sample that must be adequately supported by the test fixture. As an example of the magnitude of these forces, a $1 \mathrm{~m}$ coiled sample carrying $3000 \mathrm{~A}$ in a magnetic field of $6 \mathrm{~T}$ experiences a total Lorentz force of $18,000 \mathrm{~N}(4,037 \mathrm{lbs})$ or $180 \mathrm{~N} / \mathrm{cm}(103 \mathrm{lbs} / \mathrm{in})$ along its length. Failure to constrain the sample under the influence of these large forces can cause several different measurement problems, where the severity of 
the problem depends on the amount of sample motion. The circuit loop formed between an instrumented segment of the sample and its voltage tap leads in the presence of a strong magnetic field acts as a potent electrical generator. Under these conditions, even small sample motions can greatly increase the voltage signal noise. Moreover, if the sample motion is sufficient, heat generated by the motion can result in premature sample quenches. In the extreme case of inadequate sample constraint, the conductor can be irreversibly damaged by the Lorentz force. This situation is further complicated by the need to maintain good thermal contact between the sample and the liquid helium bath. In other words, overzealous attempts to provide adequate mechanical support (e.g. thick epoxy overlayments) can result in inadequate thermal contact and premature sample quenches.

Also, the generation of high currents requires the use of large power supplies that often introduce a new set of measurement problems. Many of these power supplies produce significant current ripple (periodic departure from dc output level), and this varying component of the sample current can cause errors in the $I_{c}$ measurement [4]. (Another paper that calculates the effect of current ripple is included as Appendix J.) This ac component of the sample current produces a corresponding ac component of the sample voltage that is then applied to the input of the dc voltmeter. The ac voltage is present even at low current levels, i.e. those below the flux flow portion of the voltage-current curve, because of the inductive loop formed between the sample and voltage tap leads. The reaction of the dc voltmeter depends on the amplitude of the ac voltage. At low amplitudes, for the two meters that have been tested, the output response to an ac input signal was approximately the time average of that signal. This characteristic of the voltmeter in conjunction with the presence of current ripple can cause a reduction in the measured $I_{c}$. At low ac amplitudes this behavior has been modeled and tested to allow a quantitative estimation of its effect. However, at some ac threshold level, which depends on the particular meter being used, the meter's response to the ac voltage becomes erratic. At this ac level, the current ripple has disabled the measurement system.

Ground loops are a problem that can exist in $I_{c}$ measurement systems regardless of the size of the system's current supply; however, the effect of ground loops is often amplified in a high current system $[5,6]$. The required differential voltage output from a current supply used for $I_{c}$ measurements is typically from 3-6 V. This low differential voltage is allowed by the extremely low load resistance of the superconductor and its current leads. Although the differential voltage at the current supply's output is low, the voltage of the output with respect to earth ground (system voltage) can be quite high (e.g. $40 \mathrm{~V}$ RMS). If there is only one point in the measurement system's electrical circuitry that is coupled to ground, this high voltage does not present a problem. On the other hand, if a second point in the system is coupled to ground (a ground loop), the high system voltage provides a strong driving force for currents to flow from the system to ground. In particular, if this ground current flows through the system's voltmeters, which are often capacitively coupled to ground, the resulting spurious voltages can lead to inaccurate or even meaningless measurement results. The high system voltage can be reduced by connecting one point of the current supply's output circuit to Earth ground, but this may also introduce a ground 
loop. This conflict seems inherent in many high current $I_{c}$ measurement systems.

Other complexities of large conductor $I_{C}$ measurements result from the conductor's increased cross-sectional dimensions and its electromechanical characteristics. The large cross-sectional area of these conductors has several ramifications for the $I_{c}$ measurement. First, the higher current capacity that is allowed by the larger conductors necessitates longer current contacts in order to avoid excessive resistive heating of the sample. Also, the current transfer length (the minimum sample length that allows uniform distribution [7] over the conductor's cross section, of the asymmetrically injected sample current) is greater for the larger conductors. This situation is complicated by the fact that the background magnetic field is an effective impetus for current transfer. Consequently, in order to insure uniform current distribution within a sample of a reasonable length, the majority of the sample should reside in the high field region of the background magnet, with the instrumented segment of the sample centered within a relatively small portion of this region. Unfortunately, the cost of the background magnet increases exponentially with the volume of its high field region. This presents a practical limit on the size of the high field volume and, thus, on the length of the instrumented segment of the sample. Moreover, this length determines the voltage signal strength and, consequently, affects the precision and accuracy of the $I_{C}$ measurement.

The mechanical construction of large conductors, whether monoliths or cables, can introduce spatial variations in their electromagnetic properties. Large monolithic conductors usually have a rectangular cross section to allow easier winding. The unequal cross-sectional deformation that is necessary to form a rectangular conductor can cause longitudinal variations in its electromagnetic properties. A rectangular conductor also exhibits electromagnetic asymmetries associated with the direction of the applied magnetic field about its longitudinal axes. Both of these phenomena are due to the aspect ratio effect [8]. In the case of cables, the degree and type of mechanical deformation varies periodically along the length of each strand. This mechanical variation results in a corresponding electromagnetic variation. Specifically, these variations are caused by localized damage, angular effects, and aspect ratio effects $[9,10,11,12]$. In both cases, monoliths and cables, these spatial variations complicate the $I_{c}$ measurement.

The measurement of large conductor critical currents involves all of the complexities associated with small conductors, many of which are amplified in the case of large conductors, as well as some problems that are unique to the large conductors. A large-conductor $I_{c}$ test method must be specific enough to address all of these problems while maintaining enough generality to be of practical benefit. A large conductor test method that is specific to SSC (Superconducting Super Collider) cables has been proposed by Brookhaven National Laboratory [13]. 


\section{E. Critical-Current Measurements on Sample \\ Used in AC Loss Round Robin}

The VAMAS Technical Working Party requested that we make critical-current measurements on the U.S. wire used in the ac loss round robin. These critical currents will be useful for analyzing and comparing the ac loss data. These $\mathrm{I}_{c}^{\prime}$ 's are reported at various applied magnetic fields, at $4.2 \mathrm{~K}$, and with a critical-current criterion of $0.1 \mu \mathrm{V} / \mathrm{cm}$ (see Table I-1 and Figure I-1). The measurements were made on a straight sample in a radial-access, split-pair magnet. The estimated uncertainty of the $I_{c}$ values is $\pm 2 \%$.

Table I-1. Critical Currents at Various Applied Magnetic Fields.

\begin{tabular}{|c|c|c|c|c|c|c|c|c|c|c|c|}
\hline$\mu_{0} \mathrm{H}, \mathrm{T}$ & 0.0 & 0.5 & 1 & 2 & 3 & 4 & 5 & 6 & 7 & 8 & 9 \\
\hline$\left[\begin{array}{ll}c & A\end{array}\right.$ & 1675 & 1344 & 1101 & 827 & 675 & 566 & 473 & 384 & 293 & 197 & 101 \\
\hline
\end{tabular}




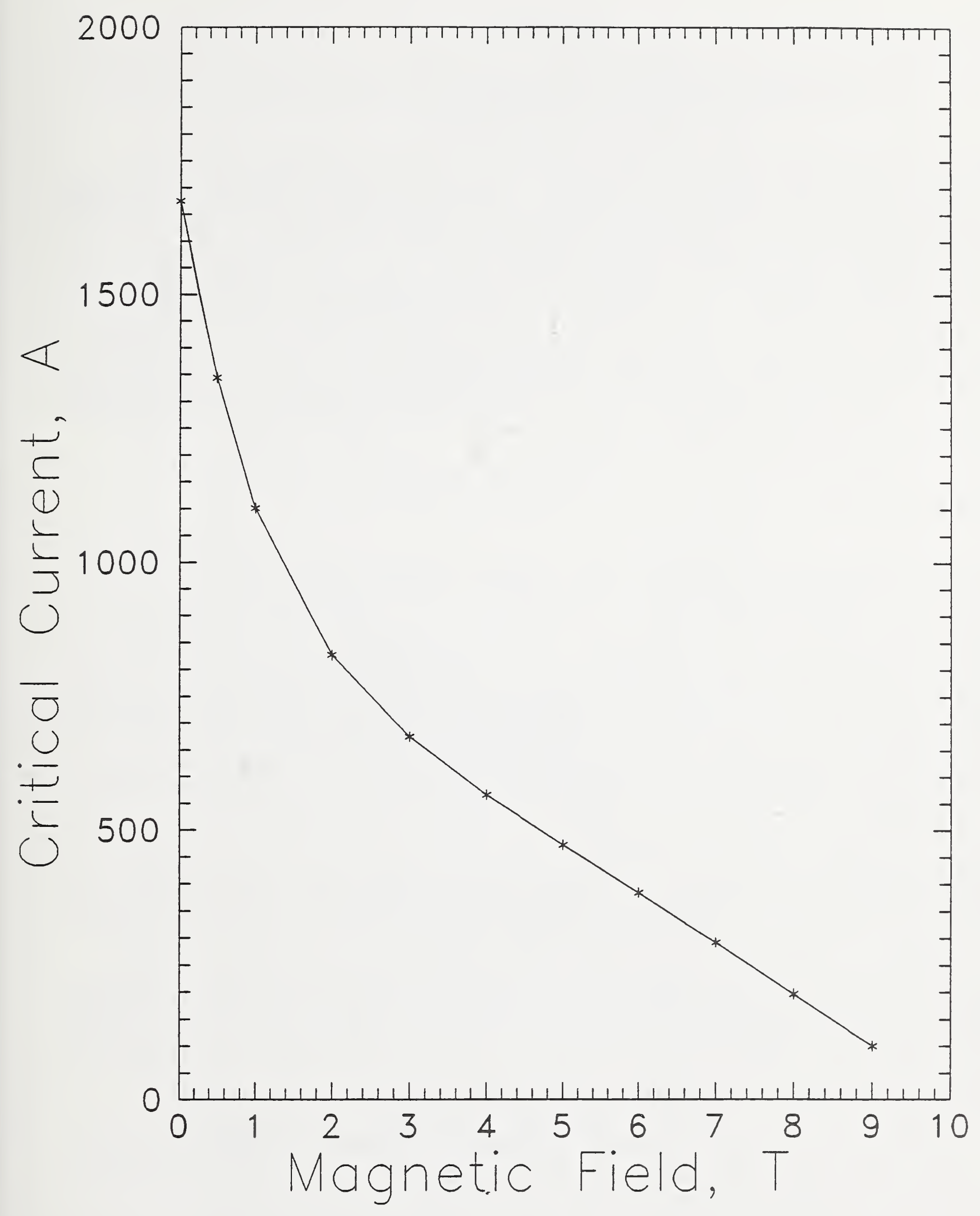

Figure I-1. Critical current versus applied magnetic field at $4.2 \mathrm{~K}$ and a critical-current criterion of $0.1 \mu \mathrm{V} / \mathrm{cm}$. 


\section{AC LOSSES}

\section{A. Short-Range Variations in Magnetic Hysteresis Loss of Multifilamentary $\mathrm{Nb}_{3} \mathrm{Sn}$}

We have experimentally investigated short-range variations in magnetic hysteresis loss of multifilamentary $\mathrm{Nb}_{3} \mathrm{Sn}$. The work was a collaboration among R. B. Goldfarb (National Institute of Standards and Technology), D. B. Smathers (Teledyne Wah Chang Albany), and M. M. Steeves (Massachusetts Institute of Technology). The study was undertaken for two reasons. The first is that measurements of hysteresis loss often use small, individually reacted samples. We want to be confident that a small sample of individually reacted wire is representative of a larger length of wire. By measuring the losses for adjacent samples, we were able to quantify variations attributed to metallurgy. The second reason is that we wished to determine the best way to react samples that are metallurgically uniform. We compared samples prepared with long "cold tails" and samples reacted with short "hot tails." In addition, we estimated precision as a function of several measurement variables.

Hysteresis is the major loss mechanism in superconductors when fields are cycled. It arises from irreversibility, owing to flux vortex pinning, of the penetration of supercurrents into the conductor. As the field is cycled, the penetrated supercurrents do not recede. Instead, oppositely circulating supercurrents enter from the surface as new flux vortices are created. In addition to energy dissipation, there is more information that can be extracted from these measurements of magnetization versus field. The width of the hysteresis loop is related to the critical current density for a given filament diameter. The remanent magnetization at zero field gives an indication of the residual field that could be expected in a superconducting magnet constructed with the wire. Time dependence of the magnetization, on a scale of seconds, gives information on flux creep.

Multifilamentary $\mathrm{Nb}_{3} \mathrm{Sn}$ is a metallurgically complicated system. The intermetallic compound is very brittle and must be formed in situ. The critical current of the reacted wire is very sensitive to mechanical strain. Tin-core $\mathrm{Nb}_{3} \mathrm{Sn}$ wire was reacted on $8 \mathrm{~mm}(5 / 16-18)$ and $5 \mathrm{~mm}(10-24)$ threaded stainless steel rods. Long "cold tails" extended out of the reaction furnace. The coiled wire was unscrewed from the rods after reaction. Four samples, each about $30 \mathrm{~cm}$ in length, were cut from each coil. The ends of each sample coil were cut with a slitting saw to avoid crushing the filaments together. The samples were soaked in phosphoric acid rust remover ("Naval Jelly") to clean the surfaces without etching away the copper matrix. The samples' density and volume were determined by Archimedes' principle. By etching some samples in dilute nitric acid, we determined that, even with the more tightly wound samples, the $\mathrm{Nb}_{3} \mathrm{Sn}$ filaments were not broken.

We used a vibrating-sample magnetometer to measure the hysteresis losses. A feature of this method is that it requires only small samples compared to magnetometers based on flux integration. For measurements in transverse field, the composite wire is threaded onto a plastic screw sample holder, and 
the field is applied along the axis of the sample coil. The temperature of measurement was $4 \mathrm{~K}$. We calibrated the magnetometer with $\mathrm{Ni}$ wire wound in the same geometry as the superconductor samples. Maximum applied fields were typically $2.4 \mathrm{MA} / \mathrm{m}$ ( $3 \mathrm{~T}$ in free space). The field was stepped.

Magnetization was measured after any matrix eddy currents were allowed to decay. Magnetization was calculated as magnetic moment per unit volume of wire (not per unit volume of $\mathrm{Nb}_{3} \mathrm{Sn}$ ). The area of the hysteresis loop corresponds to the loss. The signal from the empty sample holder and rod is diamagnetic, about one tenth the signal when the sample is measured. However, the sample holder is nonhysteretic. Therefore the hysteresis loss and width of the loop are not affected.

We measured the variations in hysteresis losses for contiguous samples of multifilamentary $\mathrm{Sn}$-core $\mathrm{Nb}_{3} \mathrm{Sn}$ reacted as a single coil. The characteristics of the wire are given in Table II-1.

Table II-1. Characteristics of $\mathrm{Nb}_{3} \mathrm{Sn}$ wire

\begin{tabular}{ll}
\hline \hline $\mathrm{Nb}$ alloy & $1 \mathrm{wt} .8 \mathrm{~T}$ \\
Diffusion barrier & vanadium \\
Tin diffusion method & Sn core, modified jelly roll \\
Wire diameter & $0.78 \mathrm{~mm}$ \\
Fraction Cu stabilizer & 0.54 \\
Local Cu/Nb ratio & $1.3 \mathrm{~cm}$ \\
Filament diameter & $3-3.5 \mu \mathrm{m}$ \\
Approximate number of filaments & 4000 \\
Number of filament subelements & 18 \\
\hline
\end{tabular}

The variation in hysteresis loss attributed to short-range metallurgical variations (over about one meter) in the wire is less than $2.5 \%$. In addition to metallurgical variations in the samples, the other sources of variation, listed in Table II-2, were examined and quantified.

One sample coil was stretched about $25 \%$ and then compressed. The winding radius was increased slightly too. The hysteresis loss increased by 0.28 . Unlike critical current, hysteresis loss appears to not be very sensitive to strain.

Table II-2. Limits to precision

\begin{tabular}{ll}
\hline \hline Metallurgical variations & $2.5 \%$ \\
Volume determination & $0.2 \%$ \\
Sample centering sensitivity & $0.6 \%$ \\
Day-to-day repeatability & $0.3 \%$ \\
Sample ends sawed vs. cut & $0.5 \%$ \\
Thread size and pitch & $4.0 \%$ \\
\hline \hline
\end{tabular}


Two wire samples were reacted with short welded ends instead of long cold tails. The purpose of welded tails or long tails is to prevent loss of $\mathrm{Sn}$ from the cut ends of the wires during reaction. The loss for one sample with apparently good welds, the "reference" sample, was as expected. The loss for the second sample was $30 \%$ less. The ends of this sample were trimmed and the loss was remeasured. This time the loss was 808 greater than that of the reference sample. It is possible that improperly welded ends allowed $\mathrm{Sn}$ to be lost from the sample. It is important to weld ends carefully when using this method to prepare samples.

This work was presented at the Applied Superconductivity Conference, 21-25 August 1988, San Francisco, paper MC-6.

\section{B. VAMAS AC Loss Interlaboratory Comparison}

R. B. Goldfarb was the NIST participant in the VAMAS round-robin on ac losses in multifilamentary $\mathrm{Nb}-\mathrm{Ti}$. Magnetic hysteresis losses were measured for samples of the "U.S. wire" and the "Japan A wire." The characteristics of the wires are given in Table II-3.

Samples of the wires were wound on the threads of a 5-mm diameter nylon screw. Because the Japan A wire had a small diameter, three strands were wound together. The magnetic field was applied along the axis of the sample coil, approximately transverse to the wire axis. Magnetization was measured at $4 \mathrm{~K}$ with a vibrating-sample magnetometer. The magnetometer was calibrated with pure nickel wire wound in the same geometry as the samples. The fields were stepped rather than ramped. The area of the loops of magnetization versus field corresponds to the magnetic hysteresis loss. The results are exhibited in Table II-4. The applied field for each loop ranged from $+B_{m}$ to $-B_{m}$, where $B_{m}$ is the maximum value indicated in the table. The estimated precision of each measurement is given.

Table II-4 gives the hysteresis loss per total sample volume. The loss per volume of $\mathrm{Nb}-\mathrm{Ti}$ is higher. For maximum fields of $3 \mathrm{~T}$, the superconductor loss is $107.4 \mathrm{~kJ} / \mathrm{m}^{3}$ for the U.S. wire, and $95.8 \mathrm{~kJ} / \mathrm{m}^{3}$ for the Japan A wire.

Table II-3. Characteristics of VAMAS wires

\begin{tabular}{lll}
\hline & U.S. Wire & Japan A Wire \\
\hline Outer diameter $(\mathrm{mm})$ & 0.742 & 0.35 \\
Filament diameter $(\mu \mathrm{m})$ & 4.6 & 6.3 \\
Number of filaments & 10,980 & 760 \\
NbTi/Matrix & $1 / 1.38$ & $21.5 / 78.5$ \\
Twist pitch (mm) & 13 & 6 \\
$\mathrm{I}_{c}$ at 3 T (A) & 653 & 50 \\
J $_{c}$ per NbTi area $\left(\mathrm{A} / \mathrm{m}^{2}\right)$ & $3.6 \times 10^{9}$ & $2.1 \times 10^{9}$ \\
Vol. NbTi (8) & 42.0 & 21.5 \\
\hline \hline
\end{tabular}


Table II-4. Hysteresis loss measurements

\begin{tabular}{|c|c|c|c|c|c|}
\hline $\begin{array}{l}\text { Wire } \\
\text { Name }\end{array}$ & $\begin{array}{l}\text { Spool } \\
\text { Number }\end{array}$ & $\begin{array}{l}\text { Sample } \\
\text { Vol. }\left(\mathrm{cm}^{3}\right)\end{array}$ & $\begin{array}{l}\text { Maximum } \\
\text { Field (T) }\end{array}$ & $\begin{array}{l}\text { Hysteresis } \\
\text { Loss }\left(\mathrm{kJ} / \mathrm{m}^{3}\right)\end{array}$ & $\begin{array}{c}\text { Precision } \\
\left(\frac{8}{8}\right)\end{array}$ \\
\hline U.S. & $\begin{array}{r}2 \\
8 \\
14\end{array}$ & $\begin{array}{l}0.1709 \\
0.1733 \\
0.0758\end{array}$ & $\begin{array}{l}3 \\
3 \\
3 \\
3 \\
1 \\
0.5\end{array}$ & $\begin{array}{l}46.9 \\
45.1 \\
45.1 \\
45.3 \\
26.9 \\
18.9\end{array}$ & $\begin{array}{l}3 \\
1 \\
1 \\
1 \\
1 \\
1\end{array}$ \\
\hline Japan & A & $\begin{array}{l}0.1924 \\
0.0962\end{array}$ & $\begin{array}{l}3 \\
3 \\
1 \\
0.5\end{array}$ & $\begin{array}{r}20.4 \\
20.6 \\
12.4 \\
7.4\end{array}$ & $\begin{array}{l}5 \\
3 \\
3 \\
1\end{array}$ \\
\hline
\end{tabular}

The results of the VAMAS interlaboratory comparison on ac losses in $\mathrm{Nb}-\mathrm{Ti}$ were discussed at two meetings in 1989: the Japan-U.S. Workshop on High-Field Superconducting Materials in February, and the International Cryogenic Materials Conference in July. The sample wires, denoted U.S. (or "D") and Japan-A (or "E"), were measured for positive field cycles of 1 to 0 to 1 tesla with no transport current. The participants were instructed to use their standard technique; there were not many restrictions placed on the measurements. The laboratories measured magnetic hysteresis using flux integration, vibrating-sample magnetometry, or calorimetry.

We analyzed the results reported at the February meeting. The mean loss for the U.S. sample was 5.08 in units of millijoules per meter length of wire. (Ordinarily, we prefer to express loss as energy per unit volume.) The standard deviation was $0.59 \mathrm{~mJ} / \mathrm{m}$. The mean loss for the Japan-A sample was $0.505 \mathrm{~mJ} / \mathrm{m}$. The standard deviation was $0.059 \mathrm{~mJ} / \mathrm{m}$. The variations were small, despite the variety of measurement methods. We compare in Figure II-1 the loss measurements for those laboratories reporting on both samples; each point represents data from one laboratory. The linear fit reveals that the variations are a consequence of systematic differences at the participating laboratories. We believe that the primary cause of variation in reported losses is inaccurate magnetic field calibration. Magnetization calibration is usually easier to achieve and is done routinely.

As part of the VAMAS program, we measured ac losses on a coil of $\mathrm{Nb}-\mathrm{Ti}$ wire designated Japan-B (or "F2") using a calorimeter on loan from Laboratoires de Marcoussis, France. Calorimetry is difficult and requires long lengths of wire. Its advantage is that all sources of dissipation are measured. An ac transport current was used, but there was no applied magnetic field. Our data (solid circles), scaled from $60 \mathrm{~Hz}$ to $50 \mathrm{~Hz}$, are shown in Figure II-2, together with the results of other participants reported at the International Cryogenic Materials Conference. 


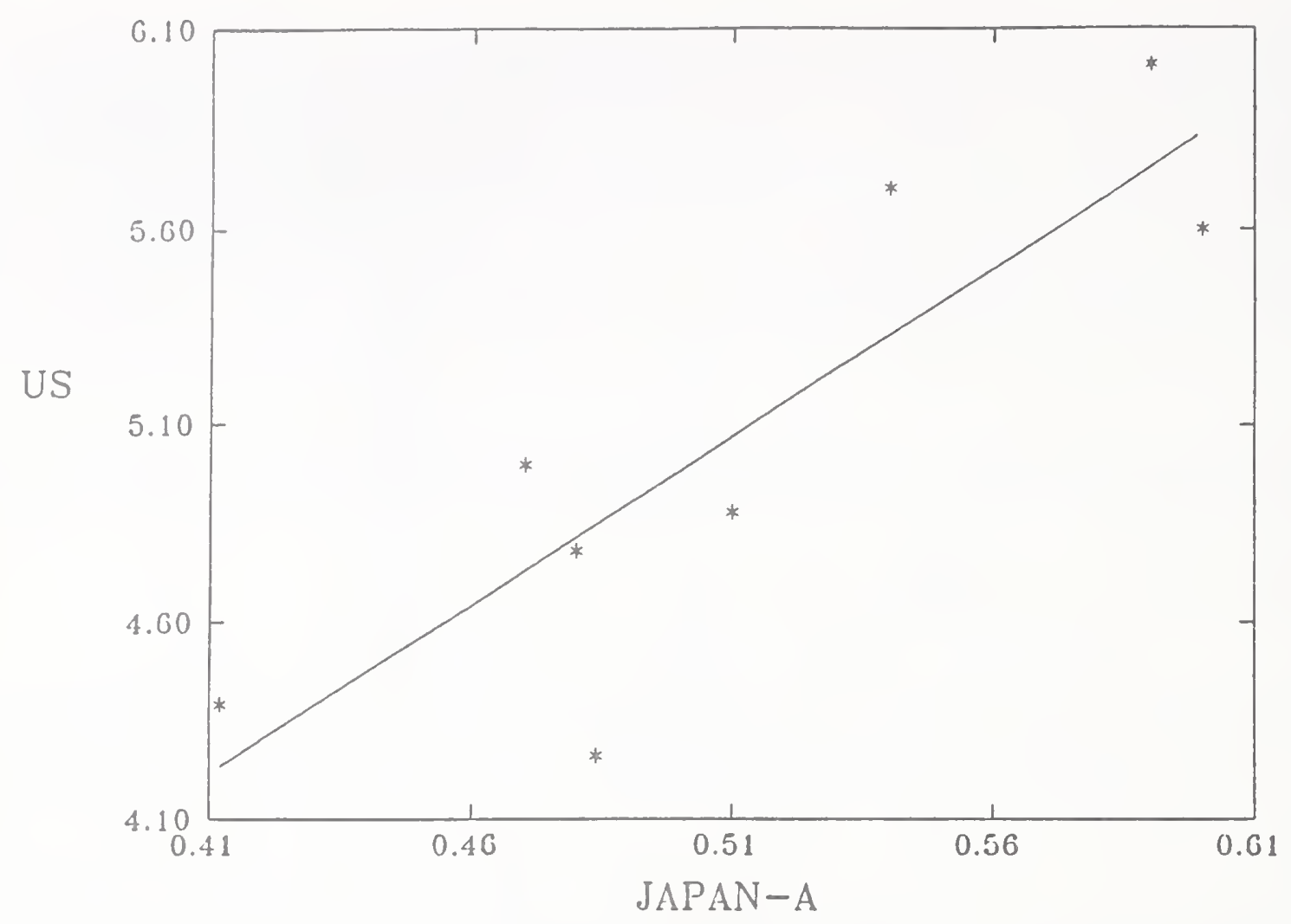

Figure II-1. Each point represents losses measured at one laboratory on two superconductor wires, "US" and "Japan-A."

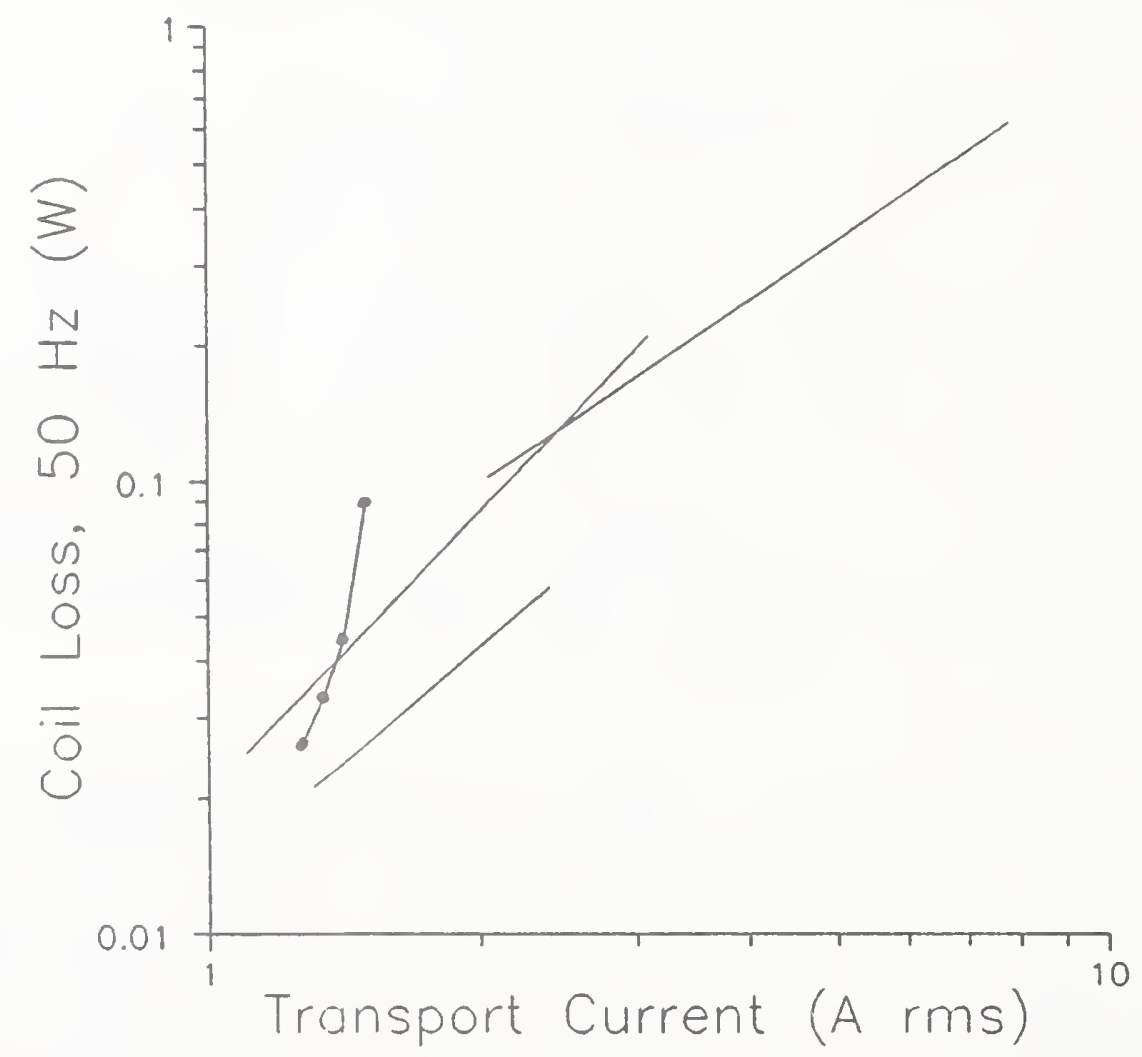

Figure II-2. Calorimetric measurement of ac power loss, scaled to $50 \mathrm{~Hz}$. The lines are fits to the data of three laboratories. The points are NIST data. 


\section{Hysteresis Loss Measurements in Support of the U.S. Demonstration Poloidal Coil Project}

We have provided measurement services to U.S. wire manufacturer Teledyne Wah Chang Albany (TWCA) to characterize their tin-core $\mathrm{Nb}_{3} \mathrm{Sn}$ conductors for ac losses. Figure II-3, from the TWCA data base, shows the hysteresis loss (measured at NIST) and the critical current density for three series of wires.

Their conductor was used by the Massachusetts Institute of Technology (MIT) in the U.S. Demonstration Poloidal Coil Project. We have measured halfcycle hysteresis losses for MIT and found that half-cycle losses were $42.6 \%$ of full-cycle losses. Appendix $\mathrm{K}$ is a paper by the MIT group on calorimetric loss measurements which incorporates our data.

We have also measured hysteresis losses in several $\mathrm{Nb}_{3} \mathrm{Sn}$ wires made by IGC Advanced Superconductors. Some of the data appear in their paper published in IEEE Transactions on Magnetics $\underline{25}, 2204$ (1989).

\section{Participation in Workshop on AC Loss in Superconducting Magnets for ITER}

At the invitation of Lawrence Livermore Laboratory, we participated in a workshop on ac losses for the International Thermonuclear Experimental Reactor (ITER) in July 1989. AC losses are expected to contribute significantly to the refrigeration load of this machine. We reviewed the major sources of ac losses for ITER: (1) magnetic hysteresis loss in the superconductor, which is controlled by filament diameter; (2) self-field loss from the pulsed transport current, which is reduced by cabling; (3) eddy-current filament coupling loss, which is reduced by twisting the filament bundle; (4) eddy currents in the superconductor matrix and conduit; and (5) magnetic hysteresis loss in the conduit.

We discussed and gave example data for three methods for characterizing a conductor for losses: (1) calorimetry, (2) magnetization as a function of field, and (3) ac susceptibility vs. temperature. While most magnetization measurements are done with fields transverse to the conductor, data are needed on longitudinal magnetization for the ITER magnets.

\section{E. Disclaimer}

Certain commercial products are identified to specify the experimental study adequately. In no case does such identification imply recommendation or endorsement, nor does it imply that the products are necessarily the best available for the purpose. 


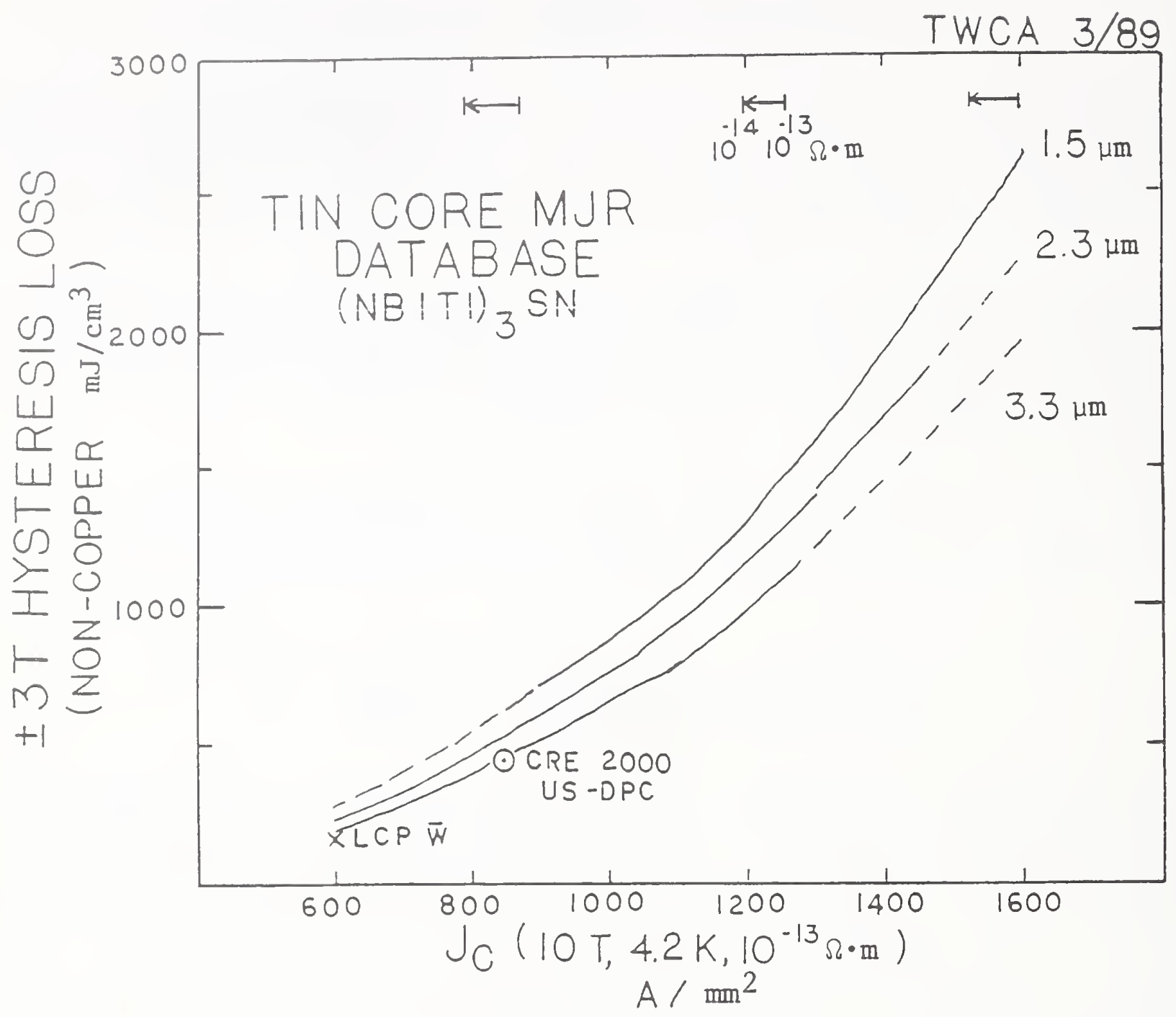

Figure II-3. Hysteresis loss and critical current density for $\mathrm{Nb}_{3} \mathrm{Sn}$ wires made by Teledyne Wah Chang Albany. Each curve is composed of many points representing different billet designs. 


\section{ADDITIONAL ACTIVITIES}

There were discussions of standards and round robin measurements at a number of meetings over the last year that are pertinent to the DoE standards program. A summary and comments from the floor are presented.

\section{A. VAMAS Technical Working Party Meeting July 8-16, 1988, Southampton, England}

There were 20 people that attended this meeting, 14 from European countries, 5 from Japan, and 1, Loren Goodrich, from the USA. Dr. David Gould (EEC) is the VAMAS coordinator. Prof. K. Tachikawa (JPN) is the chairman of both subgroups, Superconducting Materials and Cryogenic Materials. Dr. Hitoshi Wada (JPN-NRIM) is the secretary of the TWP Office.

The topics of discussion included the analysis of the $\mathrm{Nb}_{3} \mathrm{Sn}$ criticalcurrent round robin test, the $\mathrm{NbTi}$ ac loss round robin test that is now in progress, and future critical-current round robins. NIST participation in these discussions was very important since we were the sole representative for all the USA participants and our input at the meeting was fundamental in the analysis of the $\mathrm{Nb}_{3} \mathrm{Sn}$ round robin.

Prof. Tachikawa presented the first draft of the Japanese report on the analysis of all the USA, JPN, and EEC data on all three $\mathrm{Nb}_{3} \mathrm{Sn}$ wire samples. This draft had been circulated to all 24 participating laboratories well before the meeting. The results are presented in numerous plots of $I_{c} v s$. magnetic field and anonymity of each participant's data is insured by identifying the individual data sets solely by arbitrarily selected letters rather than laboratory names. Standard deviations (after excluding extraordinarily outlying results) of the measured $I_{c}$ at 10 and $15 \mathrm{~T}$ for each sample are given. NIST had sent several corrections to the USA data, suggested changes to the sections that related to our contribution, and a suggested re-write of the summary and recommendations section. Other than the wording, we at NIST felt that the report was a reasonable description of the results. The statistical analysis was somewhat misleading because the errors were not random, but our new summary emphasized the systematic error evident in these data. The NIST study of sample holder effects provided further evidence that strong systematic effects can be present in these critical current measurements.

Dr. Colin Walters prepared another analysis of the round robin data. This analysis was intended to supplement rather than to be an alternative to Prof. Tachikawa's report. The difference in the two approaches was that Dr. Walters fitted $I_{c}$ vs. magnetic field for each specimen of each participant using the approximate Kramer flux pinning law. This has some difficulties over wide field ranges, but does smooth the data and allow for interpolation or extrapolation. The results are compared in this report at a magnetic field of $11 \mathrm{~T}$. At the time of this meeting, the results of this analysis were far less complete than the Japan draft report and copies of this report had not been received by laboratories outside Europe before this meeting. 
VAMAS has its own publication medium and it is intended that both reports, Prof. Tachikawa's and Dr. Walter's, will be published therein. Dr. Gould will attempt to create a somewhat shorter summary report that will use elements from both of the larger reports. This report will be 6 to 10 pages long and is intended to be published in Cryogenics. Dr. Gould thought it would be best if we held the paper on NIST results until the first joint report is made. It may be possible to publish Dr. Gould's report and the NIST report in the same issue of Cryogenics.

Four NbTi conductors have been selected for the ac-loss round robin test, two from JPN (sample A: $6.3 \mu \mathrm{m}$ filaments and CuNi matrix, sample B: $0.5 \mu \mathrm{m}$ filaments and CuNi matrix), one from USA ( $4.6 \mu \mathrm{m}$ filaments and Cu matrix), and one from EEC (0.17 $\mu \mathrm{m}$ filaments and CuNi matrix). The two larger filament samples (JPN A and USA) have been distributed and the deadline for results was set for the end of October (subsequently changed to the end of December 1988). We were asked to collect these results from the USA participants and send them to Dr. Hitoshi Wada (secretary of the VAMAS TWP office) and to

Prof. L. van de Klundert (one of the European participants).

Both of the small filament samples were to be supplied as $20 \mathrm{~cm}$ long coils; however, few laboratories can measure a sample this large. Prof. L. van de Klundert suggested that everyone measure the sample using his method, a resonance technique using a transformer. However, it was stated that this would undermine the purpose of these tests, to compare the individual methods normally used by each participant. We need to contact the U.S. specialist to find out if a free wire sample or another coil shape could be measured.

The four selected samples will provide a range of ac losses, but the two smaller filament samples will be very difficult to measure. A $500 \mathrm{~m}$ length of the $0.5 \mu \mathrm{m}$ filament diameter sample is expected to have a loss of $1.5 \mathrm{~W}$ at 50 $\mathrm{Hz}$ and $1200 \mathrm{~V}$. The $0.17 \mu \mathrm{m}$ filament diameter sample will have a much lower loss and it is not insulated; consequently, this sample will require insulation prior to sample winding. We were asked to measure the $I_{c}$ of the USA wire over a wide range of magnetic fields, including $0 \mathrm{~T}$. The zero field $I_{c}$ will be well over $1000 \mathrm{~A}$ and will have self-field effects.

\section{B. American Society for Testing and Materials (ASTM) Meeting August 22, 1988 at ASC in San Francisco, California}

An ASTM B1.08 meeting was held in conjunction with the Applied Superconductivity Conference (ASC) in San Francisco on August 22, 1988. Although there were over 900 registrants for the conference, a notice of the meeting was posted, and an announcement of the meeting was made at the initial plenary session, only ten people attended the ASTM meeting. The meeting was held late in the evening. Unavoidably, the meeting conflicted with one poster session and a social hour; however, this was the most open time period during the conference. The decision to hold the meeting in conjunction with the ASC conference was based on the already crowded conference schedule for superconductivity. A large attendance was anticipated based on the superconductivity community's expressed realization that there are new problems with measurements and definitions, especially for the high- $\mathrm{T}_{\mathrm{c}}$ superconductors. 
In retrospect, the meeting announcement should have been specifically directed at a wider audience than the present ASTM members. In the future, it might be more productive to hold an ASTM meeting of this type at a smaller conference or appropriate workshop as a scheduled part of the agenda.

The meeting closely followed the detailed agenda with open discussion on each item. Measurement concerns relating to both conventional (NbTi and $\mathrm{Nb}_{3} \mathrm{Sn}$ ) and to high- $\mathrm{T}_{\mathrm{c}}$ superconductors were discussed. There was an overall balance in the discussions relating to conventional materials, to high- $\mathrm{T}_{\mathrm{C}}$ materials, and items common to both material types.

One topic of discussion relating to conventional materials was the extent to which existing standards (B 713-82 and B 714-82) are being used. There was a consensus among the group that these standards are completely relevant in regard to the new high- $\mathrm{T}_{\mathrm{C}}$ materials, but additional guidelines are needed. It was generally agreed upon that there are some measurement considerations unique to high- $\mathrm{T}_{\mathrm{C}}$ conductors at their present level of development. The existing ASTM standards could be appended to address these considerations without sacrificing their present integrity and commercial adequacy, but this may be premature.

There were no objections to reapproving the existing standards. Some minor changes and additions to these standards, prior to initiating the reapproval process, are being considered. Other specific comments are listed below.

- Representatives from France indicated that the ASTM standard test method was used for the basis for the development of a similar French standard. Their test method is used beyond the 600 A limit of the ASTM standard.

- There is a problem with normalizing the results of ac loss measurements: expressing the loss in terms of $\mathrm{W} / \mathrm{m}^{3}$ does not take into account the $I_{C}$ of the conductor.

- A controversy exists over the minimum copper-to-superconductor ratio needed for magnet stability.

- It should be kept in mind that, regardless of their unique properties, the high- $\mathrm{T}_{\mathrm{C}}$ materials are still superconductors and the existing standards are valid for them.

- Given the present critical-current densities for the high-T materials, it is difficult to test these conductors at effective resistivities below that of copper.

- The measured properties of the high- $\mathrm{T}_{C}$ conductors are strongly dependent upon the criteria used.

- Standards for high-T $\mathrm{C}$ conductors are premature at this time based on the rapidly changing technology and the limited commercial application at their present level of development. Should an ASTM standard be developed for high$\mathrm{T}_{\mathrm{c}}$ prior to the existence of a significant commercial market? 
- Standards for high-T materials would need to accommodate unpredictable changes in their properties; thus, this type of standard would have limited utility.

- The superconductor industry could be impaired by overspecification of their products.

- Accommodation of high- $\mathrm{T}_{C}$ conductors by the measurement standard may be premature at this time, but a Guide or a Guideline may be appropriate.

- It should not be the role of ASTM to set safety standards, but general safety concerns should be mentioned and appropriate references given. There are concerns over the toxicity and combustibility of the high- $\mathrm{T}_{\mathrm{c}}$ materials. Combustibility of fine filament conventional materials is also of concern.

- Are the present standards being used? If not, how can they be changed to be more useful in the commerce of superconductors?

- The ASTM Standard Test Method was used.in specifying the conductor for the Superconducting Super Collider, but extensive additional specifications were also necessary. Could the Standard be made more complete or would this result in an overspecification for most conductors?

- The ASTM Standard should be general so as to accommodate all applications.

- Items suggested as candidates for addition to the Standard Definitions: filament sausaging, n-value, Meissner effect, and ac losses.

- The n-value (shape of the voltage-current relationship) is an important parameter but the error in its measurement can be large.

- The level of ac loss for different conductors varies greatly depending on the application for which they were designed.

\section{C. $9^{\text {th }}$ Workshop on Niobium Titanium Superconductors January 22-25, 1989, Pacific Grove, California}

This workshop was held in Pacific Grove, California from January 22-25, 1989. L. F. Goodrich gave an audience participation talk on ASTM (American Society for Testing and Materials) Standards for superconductors with a focus on MbTi conductors. There was an overview of the existing standards and possible additions were described. One addition that was considered was a test method for large conductors, more than $600 \mathrm{~A}$. A general question that was presented was: how can the ASTM Standards be made more useful? The general consensus was that the definitions could be expanded, and we are still feeling our way in large conductor testing. Other specific comments are listed below.

- We need to wait and see if the present SSC specification is effective before considering it as an appendix to the present ASTM standard. The spring back test and other tests are new and substantially untested. Given the 
public trust in ASTM, we shouldn't base any additions on the present specifications since we are still feeling our way along.

- There used to be concern about $I_{c}$ measurements and that's why there was a lot of interest in ASTM standards in 1980 to 1982. Now it isn't clear that there is a problem.

- A wire manufacturer said his customers need self-field information.

\section{6th Japan-US Workshop on High Field Superconductors February 22-24, 1989, Boulder, Colorado}

This workshop was held in Boulder, Colorado from February 22-24, 1989. L. F. Goodrich gave an audience participation talk on ASTM Standards for superconductors with a focus on $\mathrm{Nb}_{3} \mathrm{Sn}$ conductors. There was an overview of the existing standards and possible additions were described. A general question that was presented was: how can the ASTM Standards be improved? There was no consensus on a number of key topics, such as reaction mandrel material, measurement mandrel material, and bonding method. The implication of this is that a future round robin will have to prove which method gives the best or most consistent results. Other specific comments are listed below.

- The sample holder material should not be restricted at this time; several different materials should be tested.

- Choose sample holder material and mounting technique that is easily reproducible.

- As is, the standard is not specific enough with respect to sample holder material, geometry, etc.

- The answer you get does not matter because it is a comparative measure give customer an absolute comparison.

- Suggested holder-bonding: thin walled G-10 tube, varnish, measure with force in.

- Is all varnish the same; is there variation in the G-10 material; are there ASTM standards for these materials?

- There is ambiguity in the ASTM test method with regard to the allowed differential contraction between the sample and holder.

- Practicality is a consideration, holder should be reusable.

- The problem with soldering to stainless steel is an unknown shunt current and an artificially lower n-value.

- Stainless steel holders can result in excessive precompression. 
- Reaction holders made from different materials (graphite, niobium, stainless steel, and copper) result in different $I_{c}{ }^{\prime} s$ even when measurements are made on the same measurement holder.

- There will be a problem in conductor procurement unless a consensus is reached on a measurement mandrel material to be used for acceptance testing. At present both G-10 and stainless steel are being used.

\section{E. Versailles Project on Advanced Materials and Standards (VAMAS) Technical Working Party (TWP) Meeting July 24, 1989, Los Angeles, California}

There was a VAMAS TWP meeting on superconductivity at the International Cryogenic Materials Conference in Los Angeles, California, on July 24, 1989. The topics of discussion included the analysis of the $\mathrm{Nb}_{3} \mathrm{Sn}$ critical-current round robin test, the $\mathrm{NbTi}$ ac loss round robin test, and future round robins. H. Wada, National Research Institute for Metals (Japan), is Head of the Office for the TWP, and he has printed minutes of this meeting (TWP Office Report$A(7))$. NIST made two presentations to aid in the next VAMAS round robin measurement on $\mathrm{Nb}_{3} \mathrm{Sn}$ critical current. These are included as Appendices $\mathrm{L}$ and $M$ of this report.

A detailed description of a test method for $\mathrm{Nb}_{3} \mathrm{Sn}$ critical current was prepared for the VAMAS TWP Office and is included as Appendix $N$. This measurement guideline is intended to be one of a few different measurement techniques in a second $\mathrm{Nb}_{3} \mathrm{Sn} \mathrm{I}_{\mathrm{c}}$ round robin that is being planned to determine the preferred measurement method for $\mathrm{Nb}_{3} \mathrm{Sn}$.

K. Tachikawa, the chairman of the VAMAS TWP, has set the schedule of future round robins in the TWP Office Report-A(7). A $\mathrm{Nb}_{3} \mathrm{Sn} \mathrm{I} \mathrm{I}_{\mathrm{C}}$ round robin is scheduled to start in October 1989 and end in July 1990. Also a large conductor $I_{c}$ round robin will start in February 1990. Our NbTi Reference Material might be a good candidate for these measurements. A $I_{c}$ round robin on new conductors is scheduled to start in February 1990. The first intercomparison of ac loss measurements (NbTi conductors) will be completed by December 1989. A comprehensive ac loss measurement round robin will start in April or May of 1990.

\section{F. American Society for Testing and Materials (ASTM) Meeting October 25-26, 1989, Denver, Colorado}

There was an ASTM meeting in Denver, Colorado, on October 25-26, 1989. L. F. Goodrich, now the chairman of Subcommittee B01.08 on superconductors, presented a status report on superconductivity to Committee B01 on electric conductors. The existing ASTM Standards, B713 and B714, are to be submitted for reapproval. 
D. F. Vecchia and J. D. Splett are developing the statistical model and analysis for the large-conductor, critical-current Reference Material. T. C. Stauffer and R. M. Folsom prepared samples for critical current measurements. A. N. Srivastava and L. L. Dulcie assisted with computer programming. R. L. Spomer, R. J. Loughran, and R. R. Buell assisted with the magnetization measurements. R. J. Loughran made the calorimeter measurements with assistance from R. W. Cross. J. R. Cave graciously arranged for the loan of the calorimeter. J. W. Ekin participated in discussions.

\section{REFERENCES}

[1] SRM 1457 can be obtained from: Office of Standard Reference Materials, Room B311, Chemistry Building, National Institute of Standards and Technology, Washington, DC 20234, phone (301) 975-6776.

[2] E. M. W. Leung, H. G. Arrendale, R. E. Bailey, and P. H. Michels, "Short Sample Critical Current Measurements Using a Superconducting Transformer," Adv. Cry. Eng., Vol. 33, Plenum Press, New York (1988), pp. $219-226$.

[3] K. Tachikawa, "The VAMAS Intercomparison of Critical Current Measurements in Superconducting $\mathrm{Nb}_{3} \mathrm{Sn}$ Wires," Cryogenics 29, pp. $710-715$ (1989).

[4] L. F. Goodrich and S. L. Bray, "Current-Ripple Effect on Superconductive d.c. Critical Current Measurements," Cryogenics 28, pp. 737-743 (1988).

[5] L. F. Goodrich, S. L. Bray, W. P. Dube, E. S. Pittman, and A. F. Clark, "Development of Standards for Superconductors, Interim Report Jan.-Dec. 1985," NBSIR 87-3066, National Bureau of Standards, Buulder, Colorado (April 1987).

[6] Ralph Morrison, Grounding and Shielding Techniques in Instrumentation, John Wiley \& Sons, New York (1977).

[7] J. W. Ekin, "Current Transfer in Multifilamentary Superconductors I. Theory," J. Appl. Phys., 49, 3406-3409 (1978).

[8] L. F. Goodrich, W. P. Dube, E. S. Pittman, and A. F. Clark, "The Effect of Aspect Ratio on Critical Current in Multifilamentary Superconductors," Adv. Cryo. Eng. - Materials, Vol. 32, Plenum Press, New York (1986).

[9] J. W. Ekin, L. F. Goodrich, J. Moreland, E. S. Pittman, and A. F. Clark, "Electromechanical Properties of Superconductors for High-Energy Physics Applications," NBSIR 86-3061, National Bureau of Standards, Boulder, Colorado (December 1986).

[10] L. F. Goodrich, E. S. Pittman, J. W. Ekin, and R. M. Scanlan, "Studies of NbTi Strands Extracted from Coreless Rutherford Cables," IEEE Trans. on Magnetics, MAG 23, 1642, 1987. 
[11] L. F. Goodrich and S. L. Bray, "Current Capacity Degradation in Superconducting Cable Strands," IEEE Trans. on Magn. 25(2) 1949-1952 (March 1989).

[12] J. W. Ekin, L. F. Goodrich, S. L. Bray, N. F. Bergren, and R. B. Goldfarb, "Electromechanical Properties of Superconductors for HighEnergy Physics Applications," NISTIR 89-3912, National Institute of Standards and Technology, Boulder, Colorado (November 1989).

[13] M. Garber and W. B. Sampson, "Test Methods for Cable Critical Currents and Normal State Resistance," SSC-N-488, Brookhaven National Laboratory, Upton, New York (March 1988). 


\section{APPENDIX A}

\section{PUBLICATIONS OF RESEARCH PROGRAM}

1. A. F. Clark and J. W. Ekin, "Defining Critical Current," IEEE Trans. Mag. $\underline{M A G-13}, 38-40$ (1977).

2. R. L. Powell and A. F. Clark, "Definitions of Terms for Practical Superconductors, 1. Fundamental States and Flux Phenomena," Cryogenics 17, 697-701 (1977).

3. R. L. Powell and A. F. Clark, "Definitions of Terms for Practical Superconductors, 2. Critical Parameters," Cryogenics 18, 137-141 (1978).

4. J. W. Ekin, "Current Transfer in Multifilamentary Superconductors. I. Theory," J. Appl. Phys. 49, 3406-3409 (1978).

5. J. W. Ekin, A. F. Clark, and J. C. Ho, "Current Transfer in Multifilamentary Superconductors. II. Experimental Results," J. Appl. Phys. 49 , 3410-3412 (1978).

6. A. F. Clark, J. W. Ekin, R. Radebaugh, and D. T. Read, "The Development of Standards for Practical Superconductors," IEEE Trans. Mag. MAG-15, $224-227$ (1979).

7. F. R. Fickett and A. F. Clark, "Development of Standards for Superconductors, Annual Report FY 79," NBSIR 80-1629, National Bureau of Standards, Boulder, Colorado (Dec. 1979).

8. F. R. Fickett and A. F. Clark, "Standards for Superconductors," Prof. 1979 Mech. and Mag. Energy Storage Contractors' Review Meeting, Conf790954, U.S. Dept. of Energy, Washington, DC (Dec. 1979).

9. D. T. Read, J. W. Ekin, R. L. Powell, and A. F. Clark, "Definitions of Terms for Practical Superconductors: 3. Fabrication, Stabilization, and Transient Losses," Cryogenics 19, 327-332 (1979).

10. F. R. Fickett and A. F. Clark, "Development of Standards for Superconductors," Prof. 8th Int'l. Cryo. Eng. Conf. (ICEC8), IPC Science and Technology Press (1980) pp. 494-498.

11. F. R. Fickett, S. B. Kaplan, R. L. Powell, R. Radebaugh, and A. F. Clark, "Definitions of Terms for Practical Superconductors: 4. Josephson Phenomena," Cryogenics 20, 319-325 (1980).

12. G. Fujii, J. W. Ekin, R. Radebaugh, and A. F. Clark, "Effect of Thermal Contraction of Sample Holder Material on Critical Current," Adv. Cryo. Eng. Vol. 26, Plenum Press, New York (1980) p. 589-598. 
13. G. Fujii, "Present Practices in Japan for the Measurement and Definition of Various Superconducting Parameters," Tech. Report ISSP Univ. of Tokyo, Ser. A, No. 1063 (July 1980).

14. G. Fujii, J.W. Ekin, R. Radebaugh, and A. F. Clark, "Effect of Thermal Contraction of Sample-Holder Material on Critical Current," Tech. Report ISSP Univ. of Tokyo, Ser. A, No. 1074 (Aug. 1980).

15. A. F. Clark, "Development of Standards for Practical Superconductors," Proc. Superconductivity Technical Exchange Meeting, IAPG, PIC-ELE-SC 209/1 (1980), p. 103 (presentation only).

16. R. Radebaugh, G. Fujii, D. T. Read, and A. F. Clark, "A Standards Program for ac Losses in Superconductors," Proc. IVth Int'l. Congress on Refrigeration (1980), Comm. A $\frac{1}{2}-10$ (1980), pp. 1-4.

17. F. R. Fickett, L. F. Goodrich, and A. F. Clark, "Development of Standards for Superconductors, Annual Report FY80," NBSIR 80-1642, National Bureau of Standards, Boulder, Colorado (December 1980).

18. G. Fujii, "Present Practices in Japan for the Measurement and Definition of Various Superconducting Parameters," Cryogenics 21, 21-38 (1981).

19. H. R. Segal, Z. J. J. Stekley, and T. A. DeWinter, "Development of Critical Current Measurement Standards," Proc. 1980 Applied Superconductivity Conference, IEEE Trans. Mag. MAG-17, 73-76 (1981).

20. G. Fujii, M. A. Ranney, and A. F. Clark, "Therıal Expansion of $\mathrm{Nb}_{3} \mathrm{Sn}$ and $\mathrm{V}_{3} \mathrm{Ga}$ Multifilamentary Superconducting Cables, Fiberglass-Epoxy and Cotton-Phenolic Composite Materials," Jap. J. Appl. Phys. 20, 1267-1270 (1981).

21. A. F. Clark, G. Fujii, and M. A. Ranney, "The Thermal Expa'sion of Several Materials for Superconducting Magnets," IEEE Trans. Mag. MAG-17, 2316-2319 (1981).

22. L. F. Goodrich and J. W. Ekin, "Lap Joint Resistance and Intrinsic Critical Current Measurements on a NbTi Superconducting Wire," IEEE Trans. Mag. MAG-17 69-72 (1981).

23. F. R. Fickett and L. F. Goodrich, "NBS Superconductor Standardization Program," Proc. 7th Int. Conf. on MHD Electrical Power Generation, 87-89 (October 1981).

24. L. F. Goodrich, J. W. Ekin, and F. R. Fickett, "Effect of Twist Pitch on Short-Sample V-I Characteristics of Multifilamentary Superconductors," Adv. Cryo. Eng. 28, 571-580 (1982).

25. L. F. Goodrich and F. R. Fickett, "Critical Current Measurements: A Compendium of Experimental Results," Cryogenics 22, 225-241 (1982). 
26. A. F. Clark, L. F. Goodrich, F. R. Fickett, and J. V. Minervini, "Development of Standards for Superconductors, Interim Report October 1980-January 1982," NBSIR 82-1678, National Bureau of Standards, Boulder, Colorado (July 1982).

27. Standard Definitions of Terms Relating to Superconductors, Annual Book of ASTM Standards, ASTM B713-82, Part 2.03, pp. 591-594, American Society for Testing and Materials, Philadelphia, PA (1983).

28. Standard Test Method for D-C Critical Current of Composite Superconductors, Annual Book of ASTM Standards, ASTM B714-82, Part 2.03, pp. 595-598, American Society for Testing and Materials, Philadelphia, PA (1983).

29. L. F. Goodrich, "The Effect of Field Orientation on Current Transfer in Multifilamentary Superconductors," IEEE Trans. Magn. MAG-19, 244-247 (1983).

30. L. F. Goodrich, D. F. Vecchia, E. S. Pittman, and A. F. Clark, "Critical Current Measurements on a NbTi Superconducting Wire Standard Reference Material," Adv. Cryo. Eng. 30, 953-960 (1984).

31. A. F. Clark, L. F. Goodrich, and F. R. Fickett, "Experience in Standardizing Superconductor Measurements," Journal de Physique, Colloque C1, supplement $n^{\circ} 1$, pp. C1-379-C1-382 (Tome 45, janvier 1984).

32. L. F. Goodrich, D. F. Vecchia, E. S. Pittman, J. W. Ekin, and A. F. Clark, "Critical Current Measurements on an NbTi Superconducting Wire Standard Reference Material," NBS Special Publication 260-91, National Bureau of Standards, Boulder, Colorado (September 1984).

33. A. F. Clark and L. F. Goodrich, "Characterization of a Standard Reference Superconductor for Critical Current and a Summary of Other Standard Research at NBS," Proc. Tenth International Cryogenic Engineering Conference (1984), Butterworth \& Co., Ltd., pp. 433-437 (1984).

34. L. F. Goodrich, J. V. Minervini, A. F. Clark, F. R. Fickett, J. W. Ekin, and E. S. Pittman, "Development of Standards for Superconductors, Interim Report January 1982-December 1983," NBSIR 85-3027, National Bureau of Standards, Boulder, Colorado (January 1985).

35. F. R. Fickett, "Standards for Measurement of the Critical Fields of Superconductors," NBS J. Res., 90(2), 95-113 (March-April 1985).

36. L. F. Goodrich, W. P. Dube, E. S. Pittman, and A. F. Clark, "The Effect of Aspect Ratio on Critical Current in Multifilamentary Superconductors," Adv. Cryo. Eng. - Materials 32, Plenum Press, New York (1986).

37. W. P. Dube and L. F. Goodrich, "Quench Detector Circuit for Superconducting Testing," Rev. Sci. Instrum. 57, 680-682 (1986). 
38. L. F. Goodrich and A. F. Clark, "Joint Resistivity in Superconductors," Proc. Tenth International Cryogenic Materials Conference, Published in the U.S.S.R., pp. 256-258 (1986).

39. R. B. Goldfarb and J. W. Ekin, "Hysteresis Losses in Fine Filament Internal-Tin Superconductors," Cryogenics 26, 478-481 (August 1986).

40. L. F. Goodrich, S. L. Bray, W. P. Dube, E. S. Pittman, and A. F. Clark, "Development of Standards for Superconductors, Interim Report JanuaryDecember 1985," NBSIR 87-3066, National Bureau of Standards, Boulder, Colorado (April 1987).

41. L. F. Goodrich, "Development of Standards for Superconductors, Interim Report January 1986-December 1987," NBSIR 88-3088, National Bureau of Standards, Boulder, Colorado (February 1988).

42. L. F. Goodrich and S. L. Bray, "Current Ripple Effect on Superconductive d.c. Critical Current Measurements," Cryogenics 28, 737-743 (November 1988).

43. L. F. Goodrich, S. L. Bray, and A. F. Clark, "Current-Ripple Effect on Superconductive dc Critical Current Measurements," Adv. Cryo. Eng. Materials 34, 1019-1026, Edited by A. F. Clark and R. P. Reed (Plenum Publishing Corp., 1988).

44. L. F. Goodrich, S. L. Bray, and T. C. Stauffer, "Nb $3 \mathrm{Sn}$ Critical-Current Measurements Using Tubular Fiberglass-Epoxy Mandrels," IEEE Trans. on Magn. 25(2) 2375-2378 (March 1989).

45. K. Tachikawa, K. Itoh, H. Wada, D. Gould, H. Jones, C. R. Walters, L. F. Goodrich, J. W. Ekin, and S. L. Bray, "VAMAS Intercomparison of Critical Current Measurement in $\mathrm{Nb}_{3} \mathrm{Sn}$ Wires," IEEE Trans. on Magn. 25(2) 2368-2374 (March 1989).

46. R. B. Goldfarb, D. L. Reid, T. S. Kreilick, and E. Gregory, "Magnetic Evaluation of $\mathrm{Cu}-\mathrm{Mn}$ Matrix Materials for Fine-Filament $\mathrm{Nb}-\mathrm{Ti}$ Superconductors," IEEE Trans. on Magn. 25(2) 1953-1955 (March 1989).

47. S. L. Bray, L. F. Goodrich, and W. P. Dube, "Battery Powered Current Supply for Superconductor Measurements," Rev. Sci. Instrum. 60, 261 (1989).

48. L. F. Goodrich and S. L. Bray, "Critical-Current Measurements of $\mathrm{Nb}_{3} \mathrm{Sn}$ Superconductors: NBS Contribution to the VAMAS Interlaboratory Comparison," Cryogenics 29, 699-709 (July 1989).

49. L. F. Goodrich, S. L. Bray, and T. C. Stauffer, "Thermal Contraction of Fiberglass-Epoxy Sample Mandrels and Its Effect on Critical-Current Measurements," Proceedings of the 6th Japan-U.S. Workshop on High-Field Superconducting Materials and Standard Procedures for High-Field Superconducting Materials Testing, 91-93, Edited by K. Tachikawa, K. Yamafuji, H. Wada, J. W. Ekin, and M. Suenaga (Japan 1989). 
50. L. F. Goodrich and S. L. Bray, "Proposed Study on the Effect of Sample Bonding Techniques on the measured Critical Current of $\mathrm{Nb}_{3} \mathrm{Sn}$

Superconductors," Proceedings of the 6th Japan-U.S. Workshop on HighField Superconducting Materials and Standard Procedures for High-Field Superconducting Materials Testing, 99-108, Edited by K. Tachikawa, K. Yamafuji, H. Wada, J. W. Ekin, and M. Suenaga (Japan 1989).

51. L. F. Goodrich, S. L. Bray, and T. C. Stauffer, "Thermal Contraction of Fiberglass-Epoxy Sample Holders Used for $\mathrm{Nb}_{3} \mathrm{Sn}$ Critical-Current Measurements," to be published in: Adv. Cryo. Eng.-Materials 36.

52. L. F. Goodrich and S. L. Bray, "Integrity Tests for High- $\mathrm{T}_{\mathrm{C}}$ and Conventional Critical-Current Measurement Systems," to be published in: Adv. Cryo. Eng.-Materials 36 .

53. L. F. Goodrich, R. B. Goldfarb, and S. L. Bray, "Development of Standards for Superconductors, Final Report January 1988-December 1989," to be published as NISTIR $90-\mathrm{xxxx}$, National Institute of Standards and Technology, Boulder, Colorado (1990). 



\section{APPENDIX B}

INTEGRITY TESTS FOR HIGH-T $\mathrm{T}_{C}$ AND CONVENTIONAL CRITICAL-CURRENT MEASUREMENTS SYSTEMS

L. F. Goodrich and S. L. Bray

Presented at the International Cryogenic Materials Conference Los Angeles, California

24-28 July 1989

to be published in: Advances in Cryogenic Engineering Materials, Volume 36 


\title{
INTEGRITY TESTS FOR HIGH-T $C$ AND CONVENTIONAL
}

\section{CRITICAL-CURRENT MEASUREMENT SYSTEMS*}

\author{
L. F. Goodrich and S. L. Bray
}

Electromagnetic Technology Division

National Institute of Standards and Technology

Boulder, Colorado 80303

\section{ABSTRACT}

Critical-current measurement systems must be extremely sensitive to the small differential voltage that is present across the test specimen as it changes from the zero resistance state to the flux-flow resistance state. Consequently, these measurement systems are also sensitive to interfering voltages. Such voltages can be caused by ground loops and by common mode voltages. Specific methods for testing the sensitivity of critical-current measurement systems and for detecting the presence of interfering voltages are discussed. These include a simple procedure that simulates the zero resistance state and the use of an electronic circuit that simulates the flux-flow resistance state.

\section{INTRODUCTION}

The determination of a superconductor's critical current ( $I_{c}$ ) requires the measurement of extremely low voltages, 1 on the order of $1 \mu \mathrm{V}$. Consequently, the $I_{C}$ measurement system must be quite sensitive to the resistive voltage that appears as the test specimen changes from the superconducting to the normal state, and be insensitive to other sources of voltage that might otherwise corrupt the measurement. The $I_{c}$ measurement system is susceptible to sources of interfering voltage that might be negligible for many other measurements. Ground loop and common mode voltages ${ }^{2}$ are prime examples of these sources of interference.

Because of the nebulous character of these voltages, it is often difficult to predict, based simply on the design of the measurement system, whether or not the system is prone to these problems. However, some practical test methods that are useful for checking the sensitivity and accuracy of the measurement system and for detecting the presence of interfering voltages have been developed. The test methods do not directly indicate the sources of problems; they simply indicate their presence. Consequently, the solution of these measurement problems depends on knowledge of their likely sources. As a diagnostic tool, these tests are best used for evaluating the success of specific changes that are intended to alleviate these problems.

\footnotetext{
"Contribution of NIST, not subject to copyright.
} 
There are two general test methods, the zero resistance test and the finite resistance test. The zero resistance test is used to detect the presence of interfering voltages, and the finite resistance test is used to evaluate the sensitivity and accuracy of the measurement system. Both tests can be conducted at either room or cryogenic temperature. The room temperature tests are usually more convenient, but less definitive because they do not depict the actual measurement conditions as precisely as the cryogenic tests.

Finally, the development of an $I_{c}$ measurement system is complicated by the expensive and volatile nature of the liquid cryogen. This is particularly true when the cryogen is helium and the measurement system is computer controlled. During the first $I_{c}$ measurements for a new or modified measurement system, large quantities of liquid helium are often expended during the inevitable debugging process. To address this problem, a simple electronic circuit that simulates the voltage-current (V-I) characteristic of a superconductor has been developed and tested. The simulator is an effective tool for developing the measurement system to a high level, before expending liquid helium. Also, for complex $I_{c}$ measurement systems, the simulator has proven useful as part of a pre-operation check. In this way, problems can be detected and corrected prior to transferring liquid cryogen from the storage Dewar to the measurement system cryostat.

\section{TEST MEIHODS}

\section{Finite Resistance Test}

The finite resistance test is simply a four-wire resistance measurement where the superconductor specimen is replaced in the measurement system by an appropriate copper conductor. The idea is to measure a specimen that has a known resistance to assess the accuracy and sensitivity of the measurement system. In order to closely approximate the actual measurement conditions, it is important for the copper specimen's resistance, over the length that is spanned by the voltage taps, to be similar to that of the superconductor at its critical current. This allows testing of the measurement system at an appropriate voltage and current.

\section{Zero Resistance Test}

Interfering voltages are often difficult to detect because they are not always easily distinguished from actual specimen voltages. For example, the interfering voltages can have the character of a current-transfer voltage 3 or even a flux-flow voltage. This is particularly true of the hightransition-temperature (high- $T_{C}$ ) superconductors because their V-I characteristics are not as well understood as those of the conventional, or low- $T_{C}$, materials. The zero resistance test effectively simulates an ideal superconductor where the $V$-I characteristic is $V(I)=0$.

Ideally, the test is carried out with the measurement system configured exactly as it would be for an $I_{c}$ measurement, with one exception: the voltage tap leads are not both connected to the superconductor specimen. Rather, one of the leads is connected to the specimen and the second lead is connected to the first lead close to, but not in direct contact with, the specimen. This forms a null voltage tap pair (see Fig. 1). There are situations where the null voltage tap pair should have enhanced inductance to simulate the inductance of the differential voltage tap pair. 4 In this configuration the measured voltage should be equal to zero regardess of the current carried by the specimen. Any voltage that is detected, as the current is cycled, is an interfering voltage. The important point is that all of the electrical current paths that are present for an $I_{c}$ measurement 


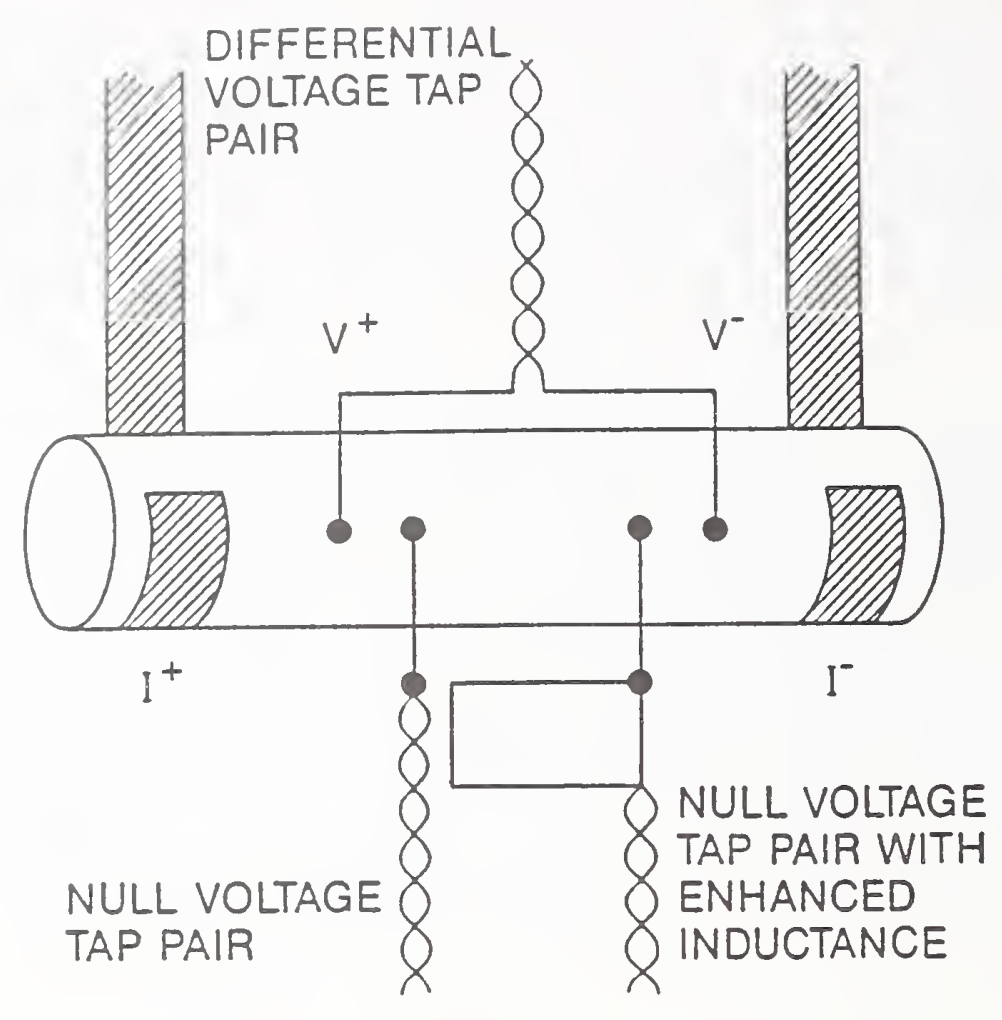

Fig. 1. Illustration of sample and lead configuration for zero and finite resistance tests.

are preserved in this configuration and the common mode voltage applied to the input terminals of the voltmeter is also the same. The only difference is that the differential voltage has been eliminated to allow easy recognition of any interfering voltages.

If the current is increased from zero to some maximum value and then decreased back to zero with a linear current ramp, equal and opposite inductive voltages will be generated for increasing and decreasing current. These voltages are easily recognized because they are of constant magnitude. In the case of a continuous data acquisition system, where the specimen voltage is recorded while the current is being continuously increased, the inductive voltages can be reduced by tightly twisting the voltage leads, but they cannot be eliminated. However, the inductive voltage is just a dc offset and, thus, it does not affect the determination of the $I_{c}$. For discrete measurement systems, where the voltage data are acquired with the current held constant at selected set points, inductive voltages are not a factor. Like inductive voltages, thermal electric voltages are always present and, if they are held constant during the data acquisition cycle, do not affect the $I_{c}$ measurement.

The interfering voltages that present a problem for $I_{c}$ measurements are those that change with changing current. These are the ground loop and common mode voltages. The zero resistance test is effective for detecting the presence of these voltages.

A more convenient, but less definitive, zero resistance test can be made at room temperature using a copper conductor, as in the finite resistance test. Once again, the voltage tap leads must be connected together and then connected to a single tap to eliminate the differential voltage. It is still important to retain as much of the actual $I_{c}$ measurement system as possible. For example, all instruments that are used 
during $I_{C}$ measurements (chart recorders, computers, and so on) should be connected for the zero resistance test. These peripheral devices often have ground connections and are sometimes the source of interfering voltages. For some measurement systems, the test fixture and specimen are not electrically isolated from ground. When this is the case, the room temperature test should be made with the copper conductor connected to ground through an appropriate resistance.

\section{Superconductor Simulator}

A simple electronic circuit was designed to simulate the intrinsic $V$-I characteristic of a superconductor. This circuit was used to characterize the response of a nanovoltmeter when subjected to a highly asymmetric periodic voltage that results from passing a dc-biased ac current through a superconductor. ${ }^{4}$ A more general application of this circuit has been to aid in the development and testing of critical-current data acquisition systems.

Another important parameter in the determination of $I_{c}$ is the measurement of the $n$ value. The parameter $n$ is defined by the approximate intrinsic voltage-current (V-I) relationship,

$$
V=V_{0}\left(I / I_{0}\right)^{n}
$$

where $I_{0}$ is a reference $I_{c}$ at a voltage criterion $V_{0}, V$ is the sample voltage, I is the sample current, and $n$ reflects the shape of the curve with typical values from 20 to 60 . A higher number means a sharper transition. In the measurement of the V-I characteristic, the sensitivity of the voltmeter is the key factor. Voltage accuracy is less significant in the determination of the $I_{c}$ for a sample with a high $n$ value. For example, with $n=30$, a voltage error of 108 translates into a 0.38 current error.

The details of the circuit design are given in Ref. 4. The input to the circuit comes from a shunt resistor connected in series with the current supply. The output current from the simulator passes through two shunt resistors, a "high output" and "low output." Typically, the nanovoltmeter being tested is connected to the low output resistor, which generates a signal in the microvolt range, and a recording instrument is connected to the high output resistor, which generates a signal that is $10^{4}$ times as large as the low output signal. Another channel of the recording instrument is connected to the analog output of the nanovoltmeter. These two channels are then compared and the measurement system may be thus characterized under conditions similar to an. $I_{c}$ measurement. The simulated values of the $I_{c}$ and n can be adjusted.

The simulator does not reproduce all of the possible elements of an actual superconductor's V-I characteristic. Current-transfer voltages and the complex voltage patterns associated with flux dynamics, for example, are not produced by the circuit. However, the simulator does produce the predominant element of a superconductor's V-I characteristic, its abrupt increase in voltage with increasing current. It also produces a very low and well defined output voltage. These two capabilities make the simulator very useful for the development and trouble shooting of $I_{c}$ measuremert systems.

\section{EXAMPLES OF TEST RESULTS}

\section{Measurement System Details}

The copper specimen used for this study was cylindrical and measured $14.6 \mathrm{~cm}$ in length and $8.9 \mathrm{~cm}$ in diameter, and it had a voltage tap 
separation of about $0.1 \mathrm{~cm}$. All of the tests made using this specimen were conducted at room temperature. Two different $I_{c}$ measurement systems were used for these tests. The details of these measurement systems are given elsewhere. 5 For both the finite resistance test and the zero resistance test, the current was steadily increased from zero to a maximum level and then steadily reduced to zero while the voltage was recorded with a digital processing oscilloscope.

\section{Finite Resistance Measurements}

Figure 2 shows the results of a finite resistance test. The hysteresis in the data is due to inductive voltage. The lower portion of the hysteresis loop is for increasing current and the upper portion is for decreasing current. The measured resistance is approximately $1.4 \mathrm{n} \Omega$. Based on the diameter of the copper conductor $(8.9 \mathrm{~cm})$ and the voltage tap separation $(0.1 \mathrm{~cm})$, this implies a copper resistivity of $0.9 \mu \Omega-\mathrm{cm}$.

The actual resistivity of the copper is probably closer to $1.7 \mu \Omega-\mathrm{cm}$. The discrepancy is probably due, primarily, to the lack of precision in the measurement of the voltage tap separation. To allow space for soldering, the voltage taps are staggered around the circumference of the conductor. Given the relatively small tap separation and the finite size of the taps, an accurate measurement of the longitudinal separation is difficult. Another source of the discrepancy is nonuniform current distribution within

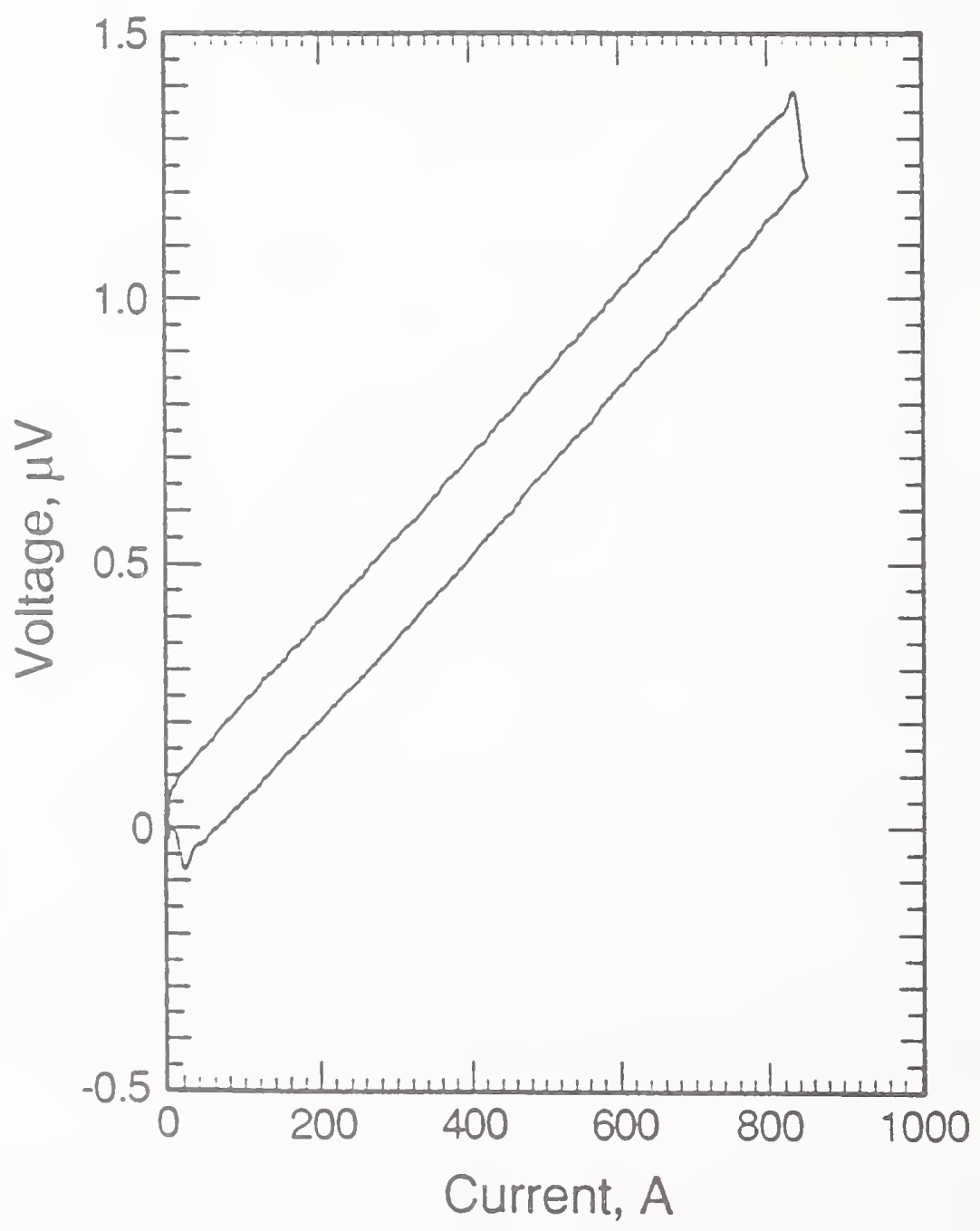

Fig. 2. Finite resistance test, voltage versus current for a copper sample. 
the conductor. For an accurate resistivity measurement, the separation between either current contact and its adjacent voltage tap should be at least five times the diameter of the conductor. This allows uniform current distribution in the region of the voltage taps. For the conductor used in these tests, the separation between the current leads and voltage tap leads is less than the diameter of the conductor. Ideally, a longer conductor with a greater tap separation would be used for this test. Nonetheless, this test demonstrates the sensitivity of the measurement and, to the extent that the measured and actual values of the copper's resistivity are in the same range, the accuracy of the measurement is demonstrated.

\section{Zero Resistance Measurements}

The results of a zero resistance measurement are shown in Fig. 3. This test was made using the same measurement system that was used for the finite resistance test. The voltage scale for this plot is nanovolts. Again the hysteresis is caused by inductive voltage. The continuous curve (upper portion of the loop) is for increasing current and the discrete data are for decreasing current. The important point is that, if the inductive voltage is subtracted from the data, the measured dc voltage is essentially equal to zero. This measurement system has a voltage noise of about $\pm 5 \mathrm{nV}$ and a voltage measurement uncertainty of about $\pm 2 \mathrm{nV} \pm 2 z$ of the signal.

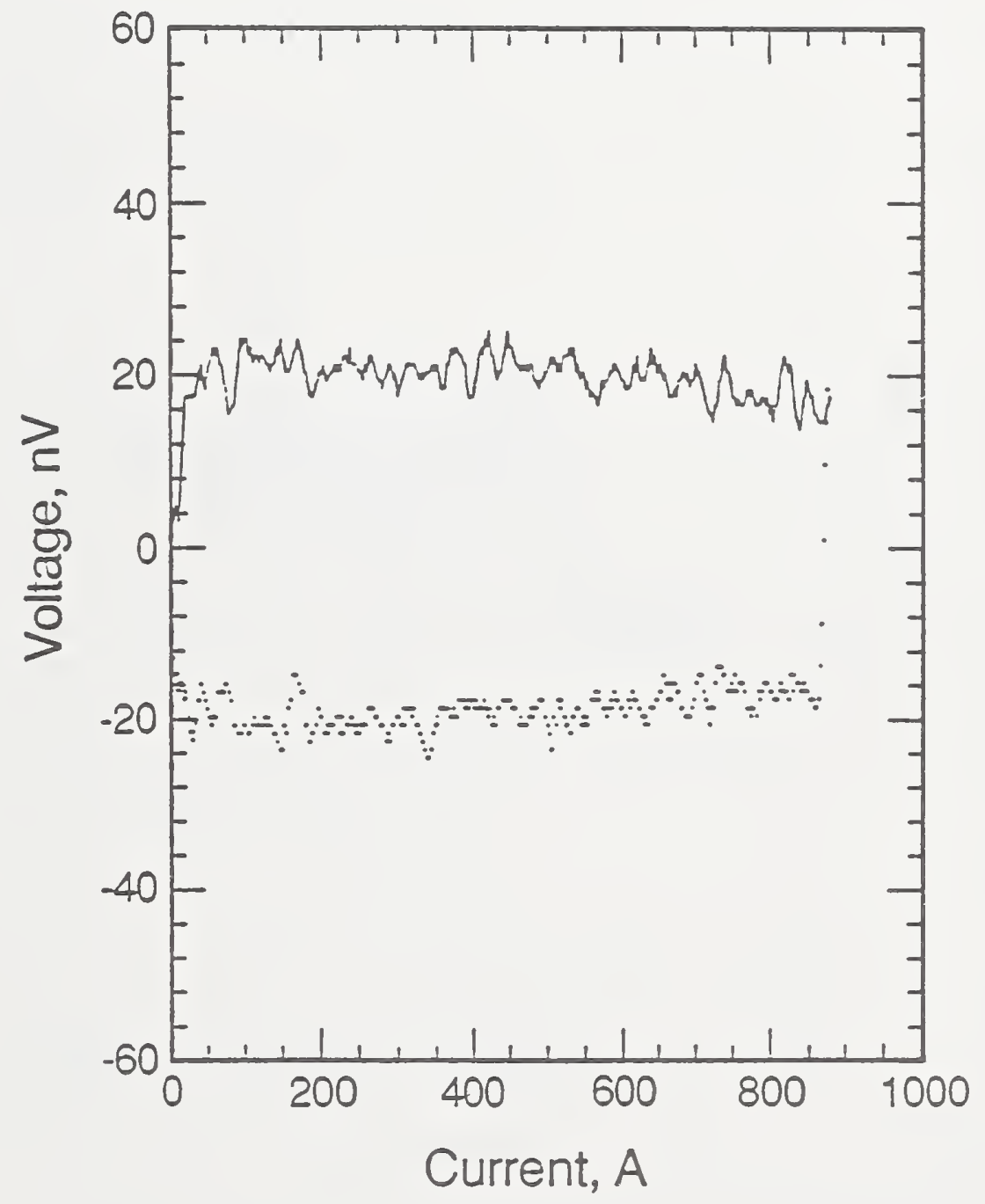

Fig. 3. Successful zero resistance test, voltage versus current for a copper sample. 
In contrast. Fig. 4 shows the results of a zero resistance test for another measurement system. In this case, the voltage scale is in microvolts and, even if the inductive voltage is subtracted from the data, the measured voltage is not equal to zero. This is an example of an interfering voltage. In an actual $I_{c}$ measurement, the interfering voltage would be detected along with any differential voltage, thus altering the $I_{C}$ measurement. In fact, the abrupt increase in voltage that occurs at approximately 900 A might be mistaken for a superconducting transition. The features of the increasing current (continuous curve) are reproduced for the decreasing current (discrete points).

\section{DISCUSSION}

Other examples of integrity tests can be found in Ref. 5, where various combinations of voltmeters, power supplies, and load grounding conditions are given. These combinations can change the results significantly; a voltmeter that works well with one current supply may not work with another. In general, if the load can be grounded near the test sample, the level of interfering voltages can be reduced. The resistance of the voltage tap leads can also be a factor; the higher the lead resistance is, the larger the interfering voltage.

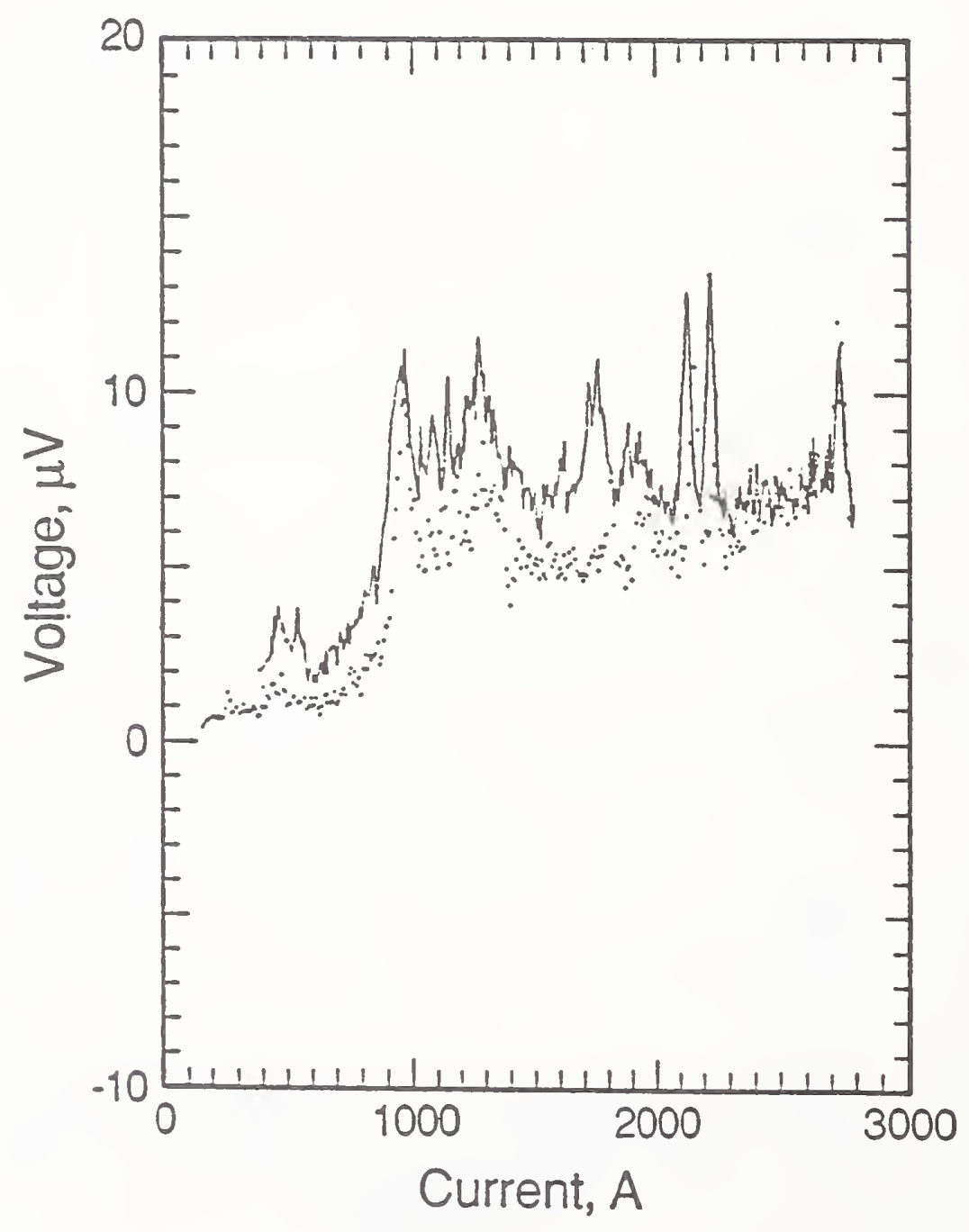

Fig. 4. Unsuccessful zero resistance test, voltage versus current for a copper sample. 
The required cross sectional area of the copper test specimen used in the finite resistance test depends on the $I_{C}$ of the superconductor, the selected $I_{C}$ criterion, and the test temperature (room or cryogenic). For high-current systems the required size of the copper specimen can become impractical. However, low-current systems may require only a copper test specimen that is comparable in cross sectional area to that of the superconductor. The length of the copper specimen is also important to ensure an accurate measurement of its resistivity. It should be long enough, in comparison with its cross sectional area, to ensure uniform current distribution in the area of the voltage taps.

\section{CONCLUSIONS}

A set of simple procedures that will test the integrity of measurement systems used for critical-current determinations on high- $I_{c}$ and conventional superconductors has been developed. These tests include a finite resistance, a zero resistance, and a superconductor voltage-current simulator. In the measurement of the critical current, voltage sensitivity is a key factor. The zero resistance test is the most effective test to detect the presence of interfering voltages such as ground loop or commonmode voltages and will determines the voltage sensitivity limit of a measurement system.

\section{ACKNOWLEDGMENTS}

The authors extend their thanks to W. P. Dube' for the idea of a zero resistance test, and to R. L. Spomer for drafting.

This work was supported by the Department of Energy, Office of Fusion Energy and Division of High Energy Physics.

\section{REFERENCES}

1. I. F. Goodrich and F. R. Fickett, Critical current measurements: a compendium of experimental results Cryogenics 22:225 (1982).

2. Ralph Morrison, "Grounding and Shielding Techniques in Instrumentation," John Wiley \& Sons, New York (1977).

3. J. W. Ekin, Current transfer in multifilamentary superconductors. I. theory J. Appl. Phys. 49:3406 (1978).

4. I. F. Goodrich and S. I. Bray, Curzent ripple effect on superconductive d.c. critical current measurements Cryogenics 28:737 (1988).

5. L. F. Goodrich, S. I. Bray, W. P. Dube', E. S. Pittman, and A. F. Clark, "Development of Standards for Superconductors, Interim Report January-December 1985," NBSIR 87-3066, National Bureau of Standards, Boulder, Colorado (1987). 



\section{APPENDIX C}

BATTERY-POWERED CURRENT SUPPLY FOR SUPERCONDUCTOR MEASUREMENTS

S. L. Bray, L. F. Goodrich, and W. P. Dube

published in: Review of Scientific Instruments

Volume 60(2), pp. 261-264, February 1989 


\title{
Battery-powered current supply for superconductor measurements
}

\author{
S. L. Bray, L. F. Goodrich, and W.P. Dube \\ Center for Electronics and Electrical Engineering, National Bureau of Standards, Boulder, Colorado 80303 \\ (Received 16 May 1988; accepted for publication 20 October 1988)
}

\begin{abstract}
To measure the critical current of superconductors, a high output current supply is required. In addition to high current capability, the supply should be designed to reduce ground loop problems, respond linearly to an input control signal, and minimize output noise. A current supply with these qualifications has been constructed and tested. Although the supply was originally designed for testing conventional superconductors at high current levels, it has also been successfully used in measurements on the high-critical-temperature ceramic superconductors where the maximum current output was less than $1 \mathrm{~A}$. The supply can produce 1000 A output current with a noise level of approximately 0.05 A peak-to-peak. The specifics of the current supply's design and performance are given.
\end{abstract}

\section{INTRODUCTION}

This current supply is an experimental design that has been gradually developed through continuous modification and improvement for an explicit application, critical-current measurements. These are extremely low-voltage measurements and, as such, they present special measurement problems. Specifically, the critical-current measurement requires a determination of the current level where the resistance of a superconducting sample becomes nonzero. In other words, a transition from zero voltage to finite voltage must be measured. ' Consequently, small spurious voltages (such as those resulting from ground loops $\mathrm{s}^{2.3}$ ), that might in some measurements be negligible, can become dominant in criticalcurrent measurements. Also, current noise can result in a systematic error in the measured critical current or in completely random measurement results, depending on the noise level. ${ }^{4}$ Finally, the inherent thermoelectric instability of a superconductor necessitates precise and stable current control. This instability also necessitates a sample protection device when operating at high current densities. ${ }^{5}$ These concerns are reflected in the design of this current supply; however, specifics of the design should be considered quite flexible depending on the particular application. Although the only use for which this supply has been tested is superconductor measurement, the general elements of its design may be useful in many other applications.

\section{CIRCUIT DESIGN AND PERFORMANCE}

\section{A. Input, control, and power stages}

The basic circuit is a voltage-controlled current source (see Fig. 1) consisting of three main stages: input, control, and power. The principal power source for the supply is a 4 $\mathrm{V}$ battery consisting of two $1500-\mathrm{A}-\mathrm{h}, 2-\mathrm{V}$ wet cells. This $4-\mathrm{V}$ battery supplies the output current through the power stage of the supply. Also, two 6-V wet cell batteries are used to power the control stage, and a $115-\mathrm{V}$ ac-powered/ $\pm 15-\mathrm{V}$ dc-output supply is used at the input stage of the supply circuit. The power stage of the circuit uses six Darlington transistor pairs in parallel, with each pair consisting of a 25 A (T3) and a 300-A (T4) power transistor. Each of the 300 A transistor emitters is connected to the positive bus bar through a separate $1-\mathrm{m} \Omega$ resistor. The resistors provide the required current-proportional feedback signals for the control stage. The collectors of these transistors are all connected to the 4 -V battery through a single $1-\mathrm{m} \Omega$ current limiting resistor. The collectors of the 25-A transistors are all connected directly to the 4-V battery and the bases are each connected to a series combination of a $0.3-\mathrm{mH}$ inductor and a $1-\Omega$ resistor. These inductors and resistors are beneficial in stabilizing the circuit with respect to parasitic oscillation. The opposite ends of the inductors are connected together, forming a branch point. The emitter of another 25-A (T2) transistor is connected to this branch point and provides the current to drive the Darlington pairs. This transistor amplifies the output signal from the control circuit. A precision $0.1-\mathrm{m} \Omega$ resistor connected between the negative battery terminal and the negative bus bar provides a current-proportional voltage signal for measuring the total current output of the supply.

The control stage of the circuit is based on an operational amplifier (OA2) that compares the signal from the input stage with the feedback signal from the Darlington emitter resistors and applies the resulting signal to the power stage. In the feedback path, another operational amplifier (OA3) is used as a summing amplifier in order to combine and amplify the six signals from the feedback resistors. A trim potentiometer is used on this operational amplifier to allow adjustment of the control circuits bias point. Without this capability, one of two undesirable conditions may exist, depending on the offset voltages of the operational amplifiers in the control loop. These offset voltages may result in an offset current at zero input voltage, or they may result in excessive deadband (where a significant threshold input voltage is required to produce an output current ), depending on the size and polarity of the offset voltages. The trim potentiometer can be used to correct either of these conditions. The potentiometer is adjusted so that a small deadband region exists at turn on. This adjustment ensures that there will be no offset current and that the deadband region is not excessive. In addition to providing a small margin of safety with respect to offset current, the residual deadband also masks any initial turn-on nonlinearity from the optically coupled input stage. In other words, the deadband prevents the sup- 


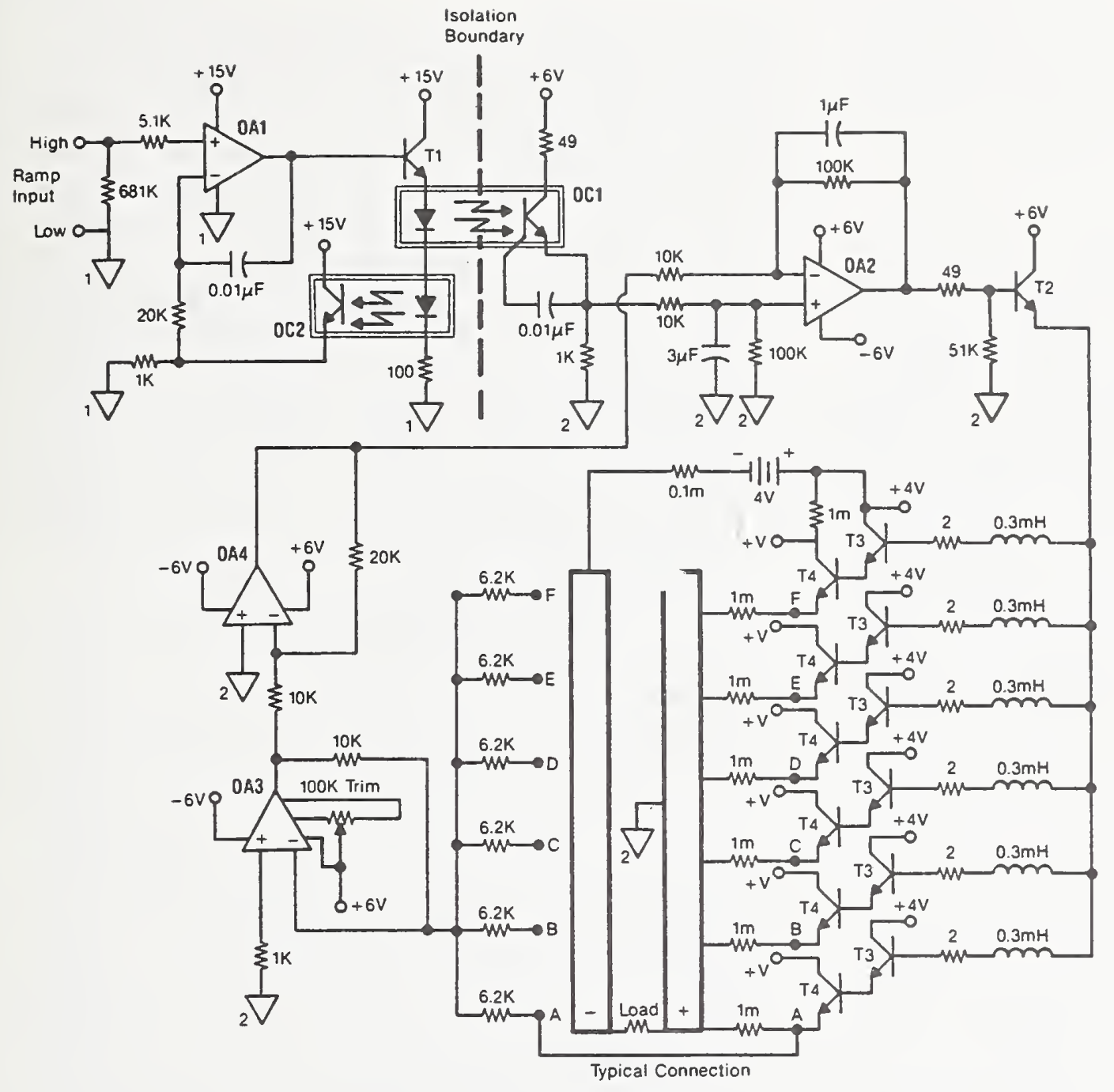

FIG. 1. Circuit diagram of the battery. powered current supply.

ply from responding to the input signal until the optical isolator is in its linear region. Since the summing amplifier inverts the feedback signal, a nother inverting amplifier (OA4) is required in the feedback path to restore the polarity of the signal. The resulting signal is then sent to the inverting input of the comparator. The comparator uses a feedback capacitor to reduce the ac gain and, thus, stabilize the circuit.

The main function of the input stage is to provide electrical isolation between the input signal generator and the supply circuit in order to a void ground loop problems. ${ }^{3}$ This isolation is achieved through the use of an optical isolator, or optocoupler $(\mathrm{OC} 1$ ). The input stage is linearized in a manner similar to that of the control stage. Again, a comparator (OA 1 ) is used to compare the reference and feedback signals. The output signal from the comparator is amplified by a single transistor ( $\mathrm{T} 1$ ). The feedback signal is supplied by a second optocoupler (OC2), which is in series with the first. This is necessary to provide electrical isolation. Moreover, the second optocoupler imitates the first and, thus, generates an appropriate feedback signal without electrically coupling the input and control stages. It is important to note that separate power supplies are used on each side of the isolation boundary (vertical dashed line on circuit diagram). Again, this avoids electrical coupling of the stages. The feed-forward optocoupler ( $\mathrm{OC} 1$ ) was found to be a significant noise source because of an unused base terminal which was picking up $60-\mathrm{Hz}$ noise. This problem was greatly reduced by connecting the base terminal to the emitter terminal with a $0.1-\mu \mathrm{F}$ capacitor. This treatment of the feedback optocoupler (OC2) resulted in oscillation; consequently, the capacitor was omitted on this optocoupler.

\section{B. Supply protection}

The battery powered current supply was not designed for continuous operation at its maximum current level. Its duty cycle is limited by the maximum power dissipation of the $300-A$ output transistors and the $1-\mathrm{m} \Omega$ collector resistor of Fig. 1. Consequently, a method of overcurrent protection that uses thermal cutoff switches on both the collector resistor and the output transistors has been designed. For redundancy, two of the output transistors are monitored with cutoff switches, and two cutoff switches are used on the collector resistor. The four cutoff switches are placed in series with the coil of a $12-\mathrm{V}$ relay and with the two $6-\mathrm{V}$ batteries. Under normal operating conditions, the thermal cutoff switches are in the closed position. This, in turn, holds the $12-\mathrm{V}$ relay in a position that connects the Darlington output stage to the control signal amplifier. $T 2$. If the temperature of any of the four thermal cutoffs exceeds its set point, the relay current is interrupted and the relay switches the Darlington input point from $T 2$ to common, which results in an interruption of the output current. After sufficient cooling. the thermal cutoff switches may be manually reset. The maximum current output of the supply depends on many factors 
including load resistance, duty cycle, and the available cooling for the output transistors. Consequently, the output capability of the supply and the specifics of the protection system will vary from one application to another.

\section{Performance}

The observed performance of the circuit with respect to output capability, linearity, current drift, and noise are described. The maximum current output of the supply in this particular application is approximately $1000 \mathrm{~A}$. The linearity of the circuits' response was measured by comparing the current supply output with the control signal input. In this case, the input signal was a linear ramp. The results of this measurement are shown in Fig. 2 where the difference between the actual output current and the ideal linear current (DELTA I) is plotted versus the dc output current (CURRENT). This plot was constructed by generating a linear fit equation from the curve of input-voltage versus output-current in its most linear region. The ideal linear current was calculated from this equation and compared with the measured current over the entire input-voltage range. This plot shows that the difference between the ideal and actual currents is less than $1 \mathrm{~A}$ for current output levels between 50 $400 \mathrm{~A}$. At the maximum output of $1000 \mathrm{~A}$ the difference is approximately $30 \mathrm{~A}$. Linearity measurements were made under a number of different conditions and these data are typical. Although this plot has been scaled to accentuate the deviation from linearity, the maximum nonlinearity represents only $3 \%$ of the measured output current.

For this application of the current supply, this level of nonlinearity is acceptable. However, for an application where a higher level of linearity is required, several elements of the present design should be considered. First, linearity of the input stage depends on a constant ratio of the large-signal current gains of the two optocouplers (OC1 and $\mathrm{OC} 2$ ). Careful matching of these two optocouplers can enhance the

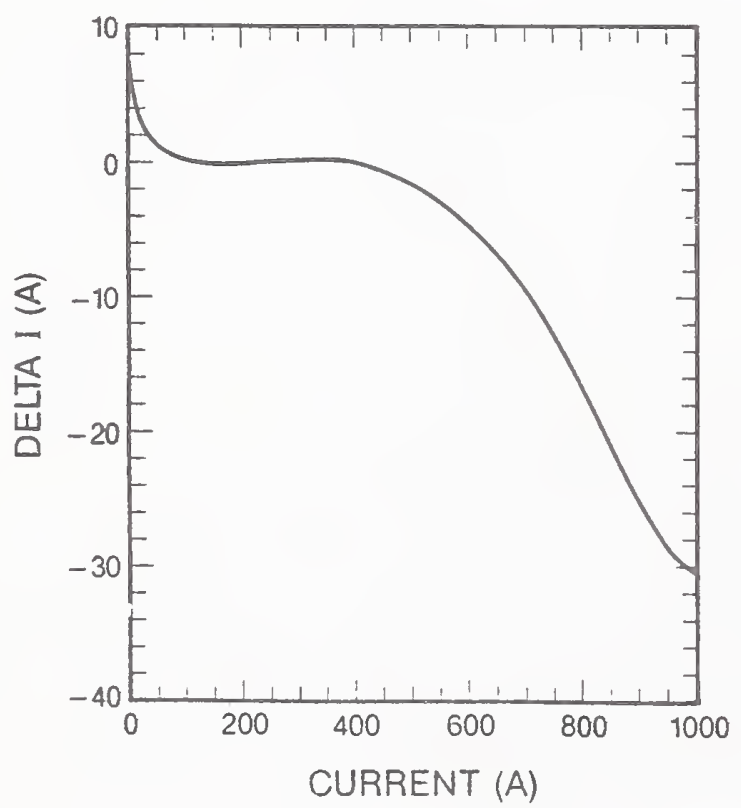

Fici. 2. l'lot of the difference between the actual outpus current and the ideal lincar current wh the de output current. linearity of the supply's response. Also, the comparator of the control circuit (OA2) results in a nonlinearity due to its closed-loop configuration. Moreover, the comparator is configured as an inverting amplifier in order to limit the gain of the offset-trim signal; however, this results in an impedance reduction at the inverting input. As a result, loading of the feedback signal by the $10-\mathrm{k} \Omega$ input resistor can reduce the supply's linearity. A reduction of this resistance can reduce this nonlinearity but an increase in the offset-trim level will also result. Finally, because the feedback signal is a sum of the signals generated by six different resistors and Darlington pairs, linearity depends on perfect matching of the resistors or consistent current sharing among the transistors. The former is more easily achieved; thus, both the $1-\mathrm{m} \Omega$ emitter resistors and the $6.2-\mathrm{k} \Omega$ summing resistors should be closely matched to enhance linearity.

The current drift of the supply was measured at two nominal current levels, 50 and $200 \mathrm{~A}$. In both cases, the input signal was increased until the nominal current output was reached and then held constant until the end of the test. The input signal and the output current were continuously recorded during the test, which had a total duration of 30 min. For the 50-A test the current output increased by $0.30 \%$ after $1 \mathrm{~min}$ and by $2.1 \%$ after $30 \mathrm{~min}$. At $200 \mathrm{~A}$, the current increased by $0.18 \%$ after $1 \mathrm{~min}$ and by $0.78 \%$ after $30 \mathrm{~min}$. Several tests were conducted with different load resistances, and these results are typical of all the tests. Also, drift measurements were made under varying initial temperature conditions. These tests suggest that the current drift is thermally induced. For example, when the supply is at ambient temperature at the beginning of the test, the current drifts upward with time regardless of the nominal current output. However, if a 200-A test is conducted immediately before a 50-A test, the current drifts downward during the 50-A test as the supply temperature decreases. As evidenced by these data, a disproportionate amount of the total current drift occurs in the first minute of the test. Drift measurements that isolated the input stage's contribution to the total current drift were made. These tests showed that the initial current drift was due primarily to the input stage. This condition might be improved by closer matching of the optocouplers, and it could certainly be improved by omitting the optically coupled input stage from the design in applications where ground loops are not a problem.

The supply's performance, with respect to output noise, was tested with two load resistances. In the first case, a lowinductance, $50-\mathrm{m} \Omega$ load (twisted wire) was used. This relatively large load resistance limited the output current to 20 $\mathrm{A}$, but it also resulted in a sufficiently large current-proportional voltage signal for accurate measurement of the current ripple. The peak-to-peak current ripple was $0.01 \mathrm{~A}$ at 20-A output current. In the second case, a load resistance on the order of $1 \mathrm{~m} \Omega$ was used to allow the supply to be operated at high currents. The voltage signal from a $0.1-\mathrm{m} \Omega$ resistor was used to measure the current ripple. For this case, the measured peak-to-peak current ripple was about $0.05 \mathrm{~A}$ for output currents from 10 to $900 \mathrm{~A}$. The actual current ripple may be less than this, but, because of residual voltage amplifier noise, this was the limit of this measurement. 


\section{DISCUSSION}

Although this design has proven very successful in this particular application, many of its elements can be changed or even deleted in order to better suit a different application. For example, in some critical-current measurement systems ground loops are not a problem and, consequently, the optically coupled input stage might be omitted in order to increase the circuits linearity while decreasing its complexity. Reference 2 gives specific test methods for evaluating critical-current measurement systems. For critical-current measurements the resistance of the current supply's load (a superconductor and connecting cables) is extremely low, thus allowing the relatively low voltage of the main battery. This low battery voltage would limit the current output for a resistive load. In this case, a higher voltage battery would be required in order to maintain the supply's output capability.

A disadvantage of a battery-powered current supply is that the batteries require periodic recharging. Furthermore, if the system is used on a daily basis at high output levels, regular overnight recharging is required. These conditions lead to the danger of overcharging. Consequently, the power supply that is used for battery charging is outfitted with two adjustable 1-to-10-h timers. Because the supply is usually unattended during recharging, two timers are used to provide redundancy. In this system both timers must malfunction in order for the batteries to continue charging beyond the selected time. Originally, lead-antimony batteries were used in this supply, but they have since been replaced with lead-calcium batteries. The calcium batteries do not generate as much hydrogen and oxygen gas when charging and, as a consequence, they do not require the maintenance or present the fire hazard that conventional batteries do. However, when overcharged, the lead-calcium batteries exhibit electrolyte sivelling, which presents the danger of acid overflow.

Generally speaking, low-level measurement systems require low-noise power supplies. This is the main benefit of a battery-powered supply as compared to conventional high current supplies. Also, a battery-powered supply offers very high ground isolation, which is crucial in some systems. For any application, the supply's relatively simple design and versatility are important benefits. In many cases', the benefits of a battery-powered supply outweigh its minor disadvantages.

\section{ACKNOWLEDGMENTS}

The authors extend their thanks to E. S. Pittman for assistance with the linearized optocouplers, to R. M. Folsom for creating circuit diagrams, and to H. E. Spilman for circuit testing. This research was supported in part by the U.S. Department of Energy through the Office of Fusion Energy and the Division of High Energy Physics.

\section{APPENDIX: COMPONENT DESCRIPTIONS}

An effort was made to avoid the identification of commercial products by the manufacturer's name or number, but in some cases these products might be indirectly identified. In no instance does this identification imply endorsement by the National Bureau of Standards, nor does it imply that the particular products are necessarily the best available for that purpose.

All of the resistors used in this circuit are $1 / 8 \mathrm{~W}, 1 \%$, metal-film resistors, except the 1 and $0.1 \mathrm{~m} \Omega$ resistors, which are high current and low-temperature-coefficient resistors. All the capacitors are ceramic. The inductors are made from No. 18 insulated wire, wound on ferrite cores. The $4 \mathrm{~V}$ battery is two $2 \mathrm{~V}, 1500 \mathrm{~A}$-h, lead-calcium wet cells wired in series. The $6 \mathrm{~V}$ batteries are $8 \mathrm{~A}$-h rechargeable wet cells. The $15 \mathrm{~V}$ supply is a $115 \mathrm{~V}$ ac input, $25 \mathrm{~mA}$ output, commercial supply. OAl is a number 3140 operational amplifier; OA2 and OA4 are number 741 operational amplifiers; and $\mathrm{OA} 3$ is a number OP5 operational amplifier. The optical isolators, $\mathrm{OCl}$ and $\mathrm{OC} 2$, are both number $4 \mathrm{~N} 25$. The transistor T1 is a 2 N3904; T2 and T3 are TIP35 transistors. The output transistors, $\mathrm{T} 4$, are $500 \mathrm{~A} / 300 \mathrm{~A}$ (peak collector current/dc collector current), 625 W/400 W $\left(25^{\circ} \mathrm{C} / 100^{\circ} \mathrm{C}\right)$ power transistors.

'L. F. Goodrich and F. R. Fickett, Cryogenics 22, 225 (1982).

${ }^{2}$ L. F. Goodrich et al., National Bureau of Standards Internal Report 873066, 1987.

${ }^{3} \mathrm{Ralph}$ Morrison, Grounding and Shielding Techniques in Instrumentation (Wiley, New York, 1977).

${ }^{4}$ L. F. Goodrich, S. L. Bray, and A. F. Clark, Adv. Cryog. Eng. 34, 1019 (1988).

sW. P. Dube and L. F. Goodrich, Rev. Sci. Instrum. 57, 680 (1986). 

APPENDIX D

$\mathrm{Nb}_{3} \mathrm{Sn}$ CRITICAL-CURRENT MEASUREMENTS USING TUBULAR FIBERGLASS-EPOXY MANDRELS L. F. Goodrich, S. L. Bray, and T. C. Stauffer

IEEE Trans. on Magnetics 25(2) 2375-2378 (March 1989) 
$\mathrm{Nb}_{3} \mathrm{~S} \mathrm{n}$ CRITICAL-CURRENT MEASUREMENTS USING TUBULAR FIBERGLASS-EPOXY MANDRELS

I. F. Goodrich, S. L. Bray, and T. C. Stauffer

National Bureau of Standards

Boulder, Colorado 80303

\section{Abstract}

A systematic study of the effect of sample mounting techniques on the superconducting critical-current measurement was made in conjunction with the VAMAS (Versailles Agreement on Advanced Materials and Standards) interlaboratory comparison (round robin) measurements. A seemingly small change in mandrel geometry can result in a $40 \%$ change in the measured critical current of a $\mathrm{Nb}_{3} \mathrm{Sn}$ sample at $12 \mathrm{I}$. This is a result of a change in the conductor pre-strain at $4 \mathrm{~K}$ caused by variation in thermal contraction between thick- and thin-walled fiberglass-epoxy composite $(G-10)$ tubes. An approximate measure of the variations in thermal contraction (from room to liquid nitrogen cemperature) indicate a 0.28 greater contraction for the thick-walled tube. This difference, combined with strain sensitivity measurements, is consistent with the observed decrease in critical current. Previous publications on the thermal contraction of G-10 have addressed the plate geometry, but not the tube geometry. The contraction of a G-10 plate is highly anisotropic. The radial contraction of a tube is different than the contraction of a plate, however. because the circumferential fiberglass is put into hoop compression by the epoxy, and the resulting contraction is a competition between the two structural components. This appears to be the source of the variation in thermal contraction with tube wall thickness.

\section{Introduction}

The measurements reported here are part of a collaborative effort involving twenty-four laboratories from the European Economic Community, Japan, and the USA under the Versailles Agreement on Advanced Materials and Standards (VAMAS). The purpose is to develop critical-current $\left(I_{c}\right)$ measurement techniques for $\mathrm{Nb}_{3} \mathrm{Sn}$ superconductors that will yield consistent results at different laboratories. Comparisons of preliminary results showed a large variation in the measured $I_{C}$. In response, the NBS study was expanded to address specific measurement variables that might have caused these discrepancies.

The predominant variables that affect $I_{c}$ measure. ments of $\mathrm{Nb}_{3} \mathrm{Sn}$ wires 1,2 fall into four categories: sample reaction conditions, measurement mandrel (sample holder) material and geometry, the method used for bonding the sample to its mandrel, and damage incurred during shipping or in transferring the sample from the reaction mandrel (tubular stainless steel sample holder) to the measurement mandrel. In the last three categories, the sensitivity of $\mathrm{Nb}_{3} \mathrm{Sn}$ to mechanical

strain results in variations in the measured $I_{C} \cdot{ }^{3}$ For these measurements, similar samples were reacted at a central location under (presumably) the same conditions; consequently, the reaction variables were not suspected of being a significant factor. The measurement mandrel material and geometry can work in conjunction with the sample bonding technique to create a dominant measurement variable: the axial strain of the conductor. Under a condition where (1) the circumferential thermal contraction of the measurement mandrel is greater than the axial thermal contraction of the conductor and (2) the bonding method creates a strong coupling between the sample and the measurement mandrel, significant axial strain of the conductor at $4 \mathrm{~K}$ may result. This study discovered that the circumferential thermal contraction of the G-10 fiberglass-epoxy tubes used for the measurement mandrels depends on the tube's geometry, owing to its composite structure. In particular, the ratio of the tube wall thickness to its radius strongly affects its thermal contraction. Based on these observations, a systematic study was made of the effect of G-10 measurement mandrel geometry on the measured $I_{C}$.

For the measurement mandrel study, a glass-filled epoxy adhesive was used for bonding the sample to the mandrel. This technique insured the strong bond and rigid coupling between the sample and mandrel that is necessary for positive stress transmission.

\section{Experimental Details}

The $\mathrm{Nb}_{3} \mathrm{Sn}$ wire measured in this study had a diameter of $0.68 \mathrm{~mm}$ with 37 sub-bundles, each contained $150 \mathrm{Nb}$ filaments. The conductor fabrication method was an internal-tin diffusion process. A single diffusion barrier of $\mathrm{Ta}$ separated the filament region from the outer layer of $\mathrm{Cu}$. The sample reaction temperature was $700^{\circ} \mathrm{C}$ for $48 \mathrm{~h}$.

The measurement mandrels were constructed from G-10 tubes that had a cylindrical copper current contact rigidly attached to each end. Each contact had a superconductive bus bar ( $\mathrm{Cu}$ and $\mathrm{Nb}_{3} \mathrm{Sn}$ tape) connected to it. The sample was transferred from the reaction mandrel onto the measurement mandrel by carefully unthreading the sample from the reaction mandrel and slipping it onto the ungrooved measurement mandrel. The one current contact that was removed for this transfer was then reattached to the measurement mandrel and the sample was tightened onto the mandrel. Each end of the sample was then soldered onto its current contact. To avoid persistent currents, an effort was made to not have a complete superconductive loop formed by the sample and bus bar around the current contact. Three pairs of adjacent voltage taps were placed along the center of the sample. Each pair had a separation of about $10 \mathrm{~cm}$ and there was a 1 to $2 \mathrm{~cm}$ gap between adjacent pairs. The sample was bonded to the mandrel with glass-filled epoxy and allowed to cure. The epoxy coat was kept thin to enhance the heat transfer from the sample to the helium bath. This unit was then mounted in the test fixture where the electrical connections were completed.

The $I_{c}$ measurements ${ }^{4}$ were made with forward (antiparallel magnetic fields, Lorentz force in) and reverse (parallel magnetic fields, Lorentz force out) current directions to determine the effect of selffield and sample-to-mandrel bonding (strain effect). Unless otherwise stated, the $I_{c}$ values given are averages over all taps, observations, and current directions at an electric field strength criterion of $10 \mu \mathrm{V} / \mathrm{m}$. The precision of the $I_{c}$ measurement was about \pm 0.28 . A statement regarding the accuracy of these measurements is presently inappropriate because of the strong systematic effects of sample mandrels.

Another parameter that was determined during the $I_{c}$ measurement is $n$. The parameter $n$ is defined by the approximate voltage-current relationship, 
$V=V_{0}\left(I / I_{0}\right)^{n}$,

where $I_{0}$ is a reference $I_{c}$ at a voltage criterion $V_{0}, V$ is the sample voltage. I is the sample current, and $n$ reflects the shape of the curve with typical values from 20 to 60 . A higher number means a sharper transition. A lower value of $n$ can be an indication of sample damage or strain.

\section{Results}

\section{Critical Current}

A summary of the $I_{c}$ data is given in Table $I$ and in Fig. 1. The information contained in Table $I$ is the $I_{c}$ data, sample mandrel dimensions, and intercomparisons of $I_{c}$ data. The $I_{c}$ data are given at magnetic fields from 6 to $12 \mathrm{~T}$. The effects of sample mandrel geometry and thermal cycling were studied. The column headings of Table I contain key words that relate to these variables. Each column, which is labeled with a letter from $A$ to $D$, is a data set taken for a single thermal cycle. Specimens are numbered 1 and 2. Different data columns that are for the same specimens (columns $A$ and $B$ for example) indicate a thermal cycle where the specimen was measured at $4 \mathrm{~K}$, warmed to room

cemperature, again cooled to $4 \mathrm{k}$, and remeasured. In some cases, the mounting configuration of the specimen was changed between these measurements (columns $B$ and $C$ for example). The sequence of the thermal cycles is from left to right in the table.

The key words heading the columns of Table I (thick. thin, and bored) are used to indicate the measurement variables. Thick and thin refer to the ratio of wall thickness to outer radius of the sample mandrel. Under the Sample Mandrel Dimensions section of the table, these specific values are listed for each data set. Bored refers to a specimen that was originally mounted and measured on a thick walled mandrel. The specimen was then brought to room temperature (thermally cycled) and the mandrel inner diameter was bored to a larger dimension, resulting in a reduced wall thickness. The specimen's $I_{c}$ was then remeasured.

A comparison of the $I_{c}$ data in columns $A$ and $B$ is $a$ measure of the $I_{c}$ variation due to thermal cycling. The maximum variation was 0.48 at $10 \mathrm{~T}$ (the highest magnetic field for column $A$ ) as shown in the intercom. parison portion of the table $([A-B] / B)$. This small variation upon thermal cycling was representative of all observations. Column $C$ was a special case of a thin walled mandrel in that it was bored (machined with the specimen mounted on the mandrel). Because this was the same specimen as $A$ and $B$, the comparison is a systematic one. The intent of this procedure was to determine the effect of mandrel wall thickness on the measured $I_{c}$ of a particular specimen. The intercom. parison data $([B-C] / C)$ shows a strongly field-dependent variation of $I_{c}$ (indicative of a strain effect) with a maximum difference of 408 at $12 \mathrm{~T}$. This is thought to result from a change in the conductor pre-strain (at $4 \mathrm{~K}$ ) due to variation in thermal contraction between thick- and thin-walled G-10 tubes. However, machining may have changed the mandrel's outer diameter at room temperature, due to stress rellef, and, thus, the prestrain state of the conductor. More details regarding thermal contraction of G-10 measurement mandrels and the resulting $I_{c}$ degradation due to axial strain are presented below. The comparison between $C$ and $D$ showed only a small difference, 3 to $4 \%$, with very little field dependence ([C-D]/D). This indicates that the effect of boring was about equivalent to that of simply mounting a specimen on a thin walled tube.

Figure 1 is a semilogarithmic plot of $I_{c}$ versus magnetic field. This type of plot was used because
Table I. Ic Data and Mandrel Dimensions

Critical current (amperes) at $10 \mu \mathrm{V} / \mathrm{m}$

\begin{tabular}{|c|c|c|c|c|}
\hline$\mu_{0} H, I$ & $\begin{array}{c}1 \\
\text { thick } \\
\text { (A) }\end{array}$ & $\begin{array}{c}1 \\
\text { thick } \\
\text { (B) }\end{array}$ & $\begin{array}{c}1 \\
\text { thin } \\
\text { bored } \\
\text { (C) }\end{array}$ & $\begin{array}{c}2 \\
\text { thin } \\
\text { (D) }\end{array}$ \\
\hline 6 & 343.3 & 343.2 & 397.3 & 386.3 \\
\hline 7 & 268.4 & 267.6 & 318.4 & 308.9 \\
\hline 8 & 207.0 & 207.4 & 253.8 & 246.0 \\
\hline 9 & 156.5 & 156.6 & 200.1 & 193.5 \\
\hline 10 & 113.6 & 114.0 & 154.7 & 149.3 \\
\hline 11 & & 78.5 & 116.0 & 111.9 \\
\hline 12 & & 50.2 & 83.5 & 80.4 \\
\hline
\end{tabular}

Sample Mandrel Dimensions

(A)

(B)

(C)

(D)

\begin{tabular}{lrrrr}
\hline Wall, mm & 9.3 & 9.3 & 2.5 & 2.0 \\
Rad. , mm & 15.6 & 15.6 & 15.6 & 15.6 \\
Wall/Rad & 608 & 608 & 168 & 138 \\
\hline
\end{tabular}

Intercomparison of Critical Current Data

\begin{tabular}{rrrc}
$\mu_{0} H, I$ & $(A-B) / B$ & $(B-C) / C$ & $(C-D) / D$ \\
\hline 6 & 0.08 & -13.68 & 2.88 \\
7 & 0.38 & -16.08 & 3.18 \\
8 & -0.28 & -18.38 & 3.28 \\
9 & -0.18 & -21.78 & 3.48 \\
10 & -0.48 & -26.38 & 3.68 \\
11 & & -32.38 & 3.78 \\
12 & & -39.98 & 3.98
\end{tabular}

it illustrates the percentage differences between the curves. The thick curve is significantly lower than the others. Figure 2 is a semilogarithmic plot of $n$ versus magnetic field. The significant element of this plot is the lower values of $n$ for the thick curve at the higher magnetic fields. This is consistent with a strain degradation.

\section{Thermal Contraction}

Thermal contraction measurements on NEMA (National Electrical Manufacturers' Association) G-10 glass-epoxy composites have been published for the plate

geometry 5,6 but not for the tube geometry. The contraction of a G-10 plate is highly anisotropic. In the nomenclature of composites, the two directions in the plane of the fiberglass fabric are warp and fill. The direction perpendicular to the plane of the fabric is referred to as the normal direction. The thermal contraction from $293 \mathrm{~K}$ to $4 \mathrm{~K}$ is about 0.248 for the warp direction and 0.718 for the normal direction. The contraction in the fill direction is expected to be a little more than that of the warp direction. The contraction in the warp direction is dominated by the fiberglass fabric and the normal direction is dominated by the epoxy. The G-10 tubes used for measurement mandrels were rolled spirals of fiberglass fabric embedded in an epoxy matrix, rather than tubes machined from.plate material. The radial direction for a rolled tube is normal to the fabric. The radial contraction of a tube is different than the contraction of a plate however, because the circumferential fiberglass is put into hoop compression by the epoxy, and the resulting contraction is a competition between the two structural components. The dependence of the radial contraction on wall thickness is expected to be caused by this competition. 


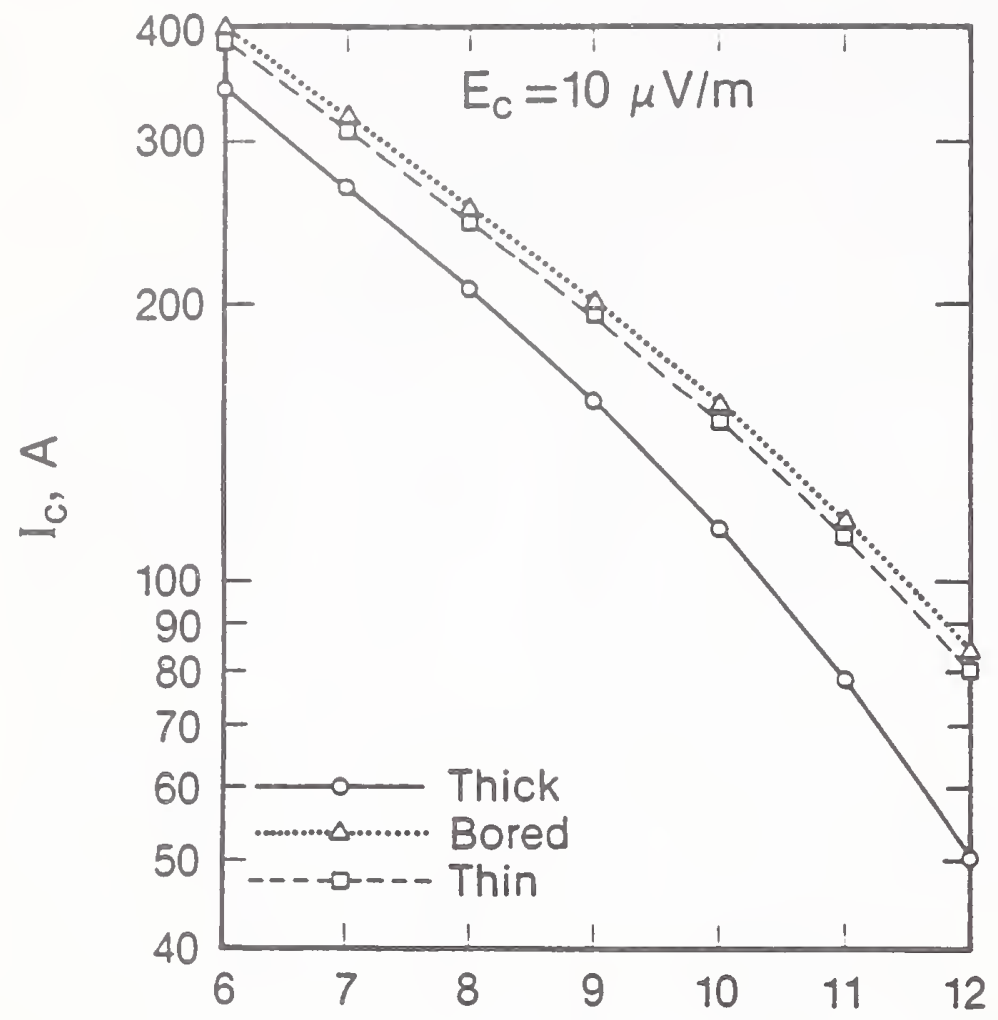

Magnetic Field, T

Figure 1. A semilogarithmic plot of $I_{c}$ at an electric field criterion of $10 \mu \mathrm{V} / \mathrm{m}$ versus magnetic field.

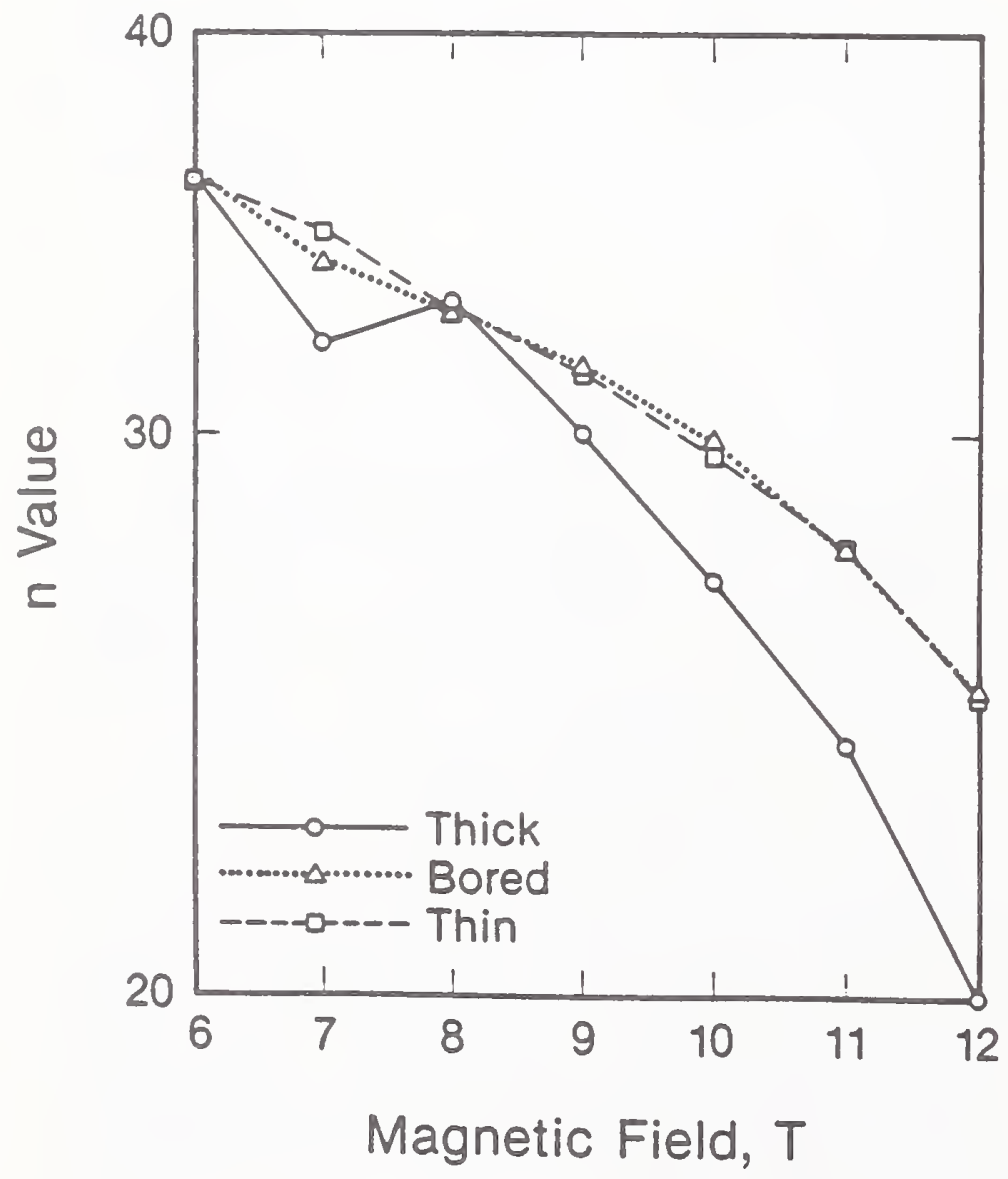

Figure 2. A semilogarithmic plot of $n$ value versus magnetic field.
A thick-walled G-10 tube was originally selected as a common sample mandrel material to allow adaptation of one size tube to the various test fixtures used by the different laboratories. However, a thick-walled tube is difficult to manufacture without delaminations that can cause irregular contraction and voids when they are machined. Also, the outer diameter can change when the inner diameter is bored. Another observation was that the radial contraction can be slightly asymmetric; this will result in an approximately elliptical rather than circular cross section at low temperatures. In order to make an approximate correction for this, the thermal contraction measurements are the average of two approximately orthogonal measurements of the tube's diameter.

As briefly mentioned above, thermal contraction measurements were made on several G-10 tubes in order to estimate the resulting conductor strain. In the interest of expediency, these measurements were limited both in accuracy and precision; nonetheless, the measurements give an indication of the variation in thermal contraction between the tubes. These tests consisted of measuring the outside diameter of the G.10 tube at room temperature, submerging the tube in liquid nitrogen, allowing it to reach thermal equilibrium, and then quickly removing it and remeasuring the diameter. These measurements were made with a precision micro. meter, but they were limited by the practical difficulties of the measurement.

The measurements showed a substantial difference between the radial thermal contraction of G.10 tubes with different wall thicknesses. The results of the thermal contraction measurements are shown in Table II. As indicated by the column headings in the right hand portion of the table, four different samples were measured. Of the four samples, the geometry of sample 1 was most like that of the actual measurement mandrels. This sample had a stepped cross-sectional geometry characterized by a longitudinal variation in its inner and outer diameters. The outside diameter of the sample was reduced at its ends and its inside diameter was enlarged at its center. This stepped geometry was necessitated by the cylindrical current contacts that were flush mounted over the ends of the mandrel. Reducing the outside diameter allowed the contacts to slip over the mandrel's ends; enlarging only the central portion of the mandrel's inside diameter allowed a reduction in the mandrel's wall thickness only in the area of the sample, while maintaining the required support in the area of the current contacts. The other three samples had constant inside and outside diameters along their lengths.

Table II. Thermal Contraction of G-10 Tubes

\begin{tabular}{lc|cc}
\hline \multicolumn{2}{l|}{ Mandrel Dimensions } & \multicolumn{2}{|c}{$\begin{array}{l}\text { Outer Diameter } \\
\text { Thermal Contraction }\end{array}$} \\
\hline $\begin{array}{l}\text { Ratio } \\
\text { Wall/Radius }\end{array}$ & $\begin{array}{c}\text { Wall } \\
\text { Thickness }\end{array}$ & $\begin{array}{c}\text { Sample } \\
\Delta d / d\end{array}$ & $\begin{array}{c}\text { Sample 2 } \\
\Delta d / d\end{array}$ \\
\hline 598 & $9.27 \mathrm{~mm}$ & 0.398 & 0.418 \\
498 & $7.70 \mathrm{~mm}$ & 0.388 & 0.368 \\
398 & $6.10 \mathrm{~mm}$ & 0.338 & 0.328 \\
288 & $4.32 \mathrm{~mm}$ & 0.308 & 0.288 \\
168 & $2.54 \mathrm{~mm}$ & 0.258 & 0.238 \\
138 & $2.03 \mathrm{~mm}$ & 0.258 & 0.248 \\
\hline Ratio & Wal1 & Sample & Sample 4 \\
Wall/Radius & Thickness & $\Delta d / \mathrm{d}$ & $\Delta d / \mathrm{d}$ \\
\hline 208 & $3.18 \mathrm{~mm}$ & 0.258 & \\
308 & $4.76 \mathrm{~mm}$ & & 0.288 \\
\hline
\end{tabular}


Samples 1 and 2 were progressively bored to larger inside diameters while samples 3 and 4 had factory diameters (not machined) and were measured to test the equivalence between factory tubes and bored tubes with respect to thermal contraction.

For each sample, the table shows the tube's wall thickness, the ratio of the tube's wall thickness to its radius in percent, and the thermal contraction of the tube between room and liquid nitrogen temperatures $(\Delta d / d)$. The data indicate that the thicker walled tubes contract more than the thinner ones. In addition, the good agreement between the data for samples 1 and 2 indicates that the contraction of the actual measurement mandrels is similar to that of simple tubes. The agreement of the data for samples 3 and 4 with those of 1 and 2 indicates that the bored tubes behave in a manner similar to that of factory tubes. Samples 1 and 2 with wall/radius ratios of 598 , 168 , and 138 have respectively similar dimensions to the thick, thin bored, and thin measurement mandrels of Iable I.

A bias in this study exists as a result of the thermally transient measurement conditions (with a systematic difference in thermal mass) and the fact that it was conducted at liquid nitrogen, rather than liquid helium, temperature. Both of these factors result in an underestimate of the actual thermal contraction between room and liquid helium tempera. cures. Based on the temperature dependence of the thermal contraction of G-10 plate, 5 the thermal contraction to $4 \mathrm{~K}$ was estimated as $-0.28 \%$ for the thin walled mandrel and -0.488 for the thick walled mandrel. The thermal contraction of the $\mathrm{Nb}_{3} \mathrm{Sn}$ conductor was only -0.218 . Consequently, the G.10 contraction may have introduced additional amounts of conductor pre-strain. The amount of compressive pre-strain might depend on several factors including the mandrel's wall thickness and the strength of the mandrel-to-conductor bond.

Considerable $I_{c}$ tensile-strain-sensitivity data were available for this sample; however, no explicit data were available for compressive strain sensitivity.

Consequently, the strain scaling $\mathrm{law}^{3}$ in conjunction with the available tensile strain data, was used to estimate the expected $I_{c}$ degradation for compressive strain. The results of these calculations predict a -338 reduction in the $I_{c}$ at $12 \mathrm{I}$ for the thick-walled specimens as compared to the thin-walled ones; the observed reduction was -408 . These measurements and calculations are only approximate, but the trend is apparent. In all cases, the thick walled samples had reduced $I_{c}$ 's and this reduction was in approximate agreement with that predicted by the strain scaling law.

\section{Conclustons}

The bonding method and the mandrel material and geometry have a significant effect on the critical current measurement of $\mathrm{Nb}_{3} \mathrm{Sn}$. Thick-walled G-10 tubes contract about 0.28 more than thin-walled G-10 from room to liquid helium temperature. Thus, the amount of differential thermal contraction between the mandrel and the sample depends on the wall thickness of the G-10. The effect of strain on the $I_{c}$ of a particular sample depends on its upper critical field. The sample tested here has an upper critical field of $-19 \mathrm{I}$ and the difference between critical current measurements on thick- and thin-walled G-10 was about 408 (predicted 338) at $12 \mathrm{~T}$. Another sample with an upper critical field of $-24 \mathrm{I}$ was also measured and, in this case, the difference between critical current measurements on thick- and thin-walled G-10 was about 178 (predicted 248) at $12 \mathrm{~T}$.
G-10 tubes that have diameters similar to that of those tested, and wall thicknesses about $20 \%$ or less of the tube's radius, are well matched in thermal contrac. tion to $\mathrm{Nb}_{3} \mathrm{Sn}$ wires and use of this cype of cube for measurement mandrels should result in a minimum of conductor strain. The scaling of this effect for tubes of various diameters has not been demonstrated, but scaling is probably accurate for a limited range of tube diameters.

\section{Acknowledgments}

The authors extend their thanks to J. W. Ekin for discussions on these results; to R. M. Folsom for sample preparation, data reduction, and plotting; to D. L. Rule for help with data plotting; to W. E. Look for assistance with the magnetic field calibration; and to the other VAMAS participants.

This work was supported by the Department of Energy, Office of Fusion Energy and Division of High Energy Physics.

An effort was made to avoid the identification of commercial products by the manufacturer's name or label, but in some cases these products might be indirectly identified by their properties. In no instance does this identification imply endorsement by the National Bureau of Standards, nor does it imply that the products are necessarily the best available for that purpose.

\section{References}

[1] L. F. Goodrich and F. R. Fickett, "Critical Current Measurements: A Compendium of Experimental Results," Cryogenics, vol. 22, pp. 225-241, May 1982 .

[2] Standard Test Method for D-C Critical Current of Composite Superconductors, Annual Book of ASTM Standards, ASTM B714-82, Part 02.03, PP. 595-98, American Society for Testing and Materials, Philadelphia, PA (1983).

[3] J. W. Ekin, "Strain Scaling Law for Flux Pinning in Practical Superconductors. Part 1: Basic Relationship and Application to $\mathrm{Nb}_{3} \mathrm{Sn}$ Conductors," Cryogenics, vol. 20, Pp. 611-624, Nov. 1980.

[4] L. F. Goodrich, "Development of Standards for Superconductors, Interim Report January 1986. December 1987," NBSIR 88-3088, National Bureau of Standards, Boulder, CO (February 1988).

[5] G. Fuj1i, J. W. Ekin, R. Radebaugh, and A. F. Clark, "Effect of Thermal Contraction of Sample Holder Material on Critical Current," Adv. Cryog. Eng. Materials, vol. 26. Pp. 589.598 , Plenum Press, New York (1980).

[6] A. F. Clark, G. Fujif, and M. A. Ranney, "The Thermal Expansion of Several Materfals for Superconducting Magnets," IEEE Trans. Mag., MAG$17,2316,1981$. 



\section{APPENDIX E}

CRITICAL CURRENT MEASUREMENTS OF $\mathrm{Nb}_{3} \mathrm{Sn}$ SUPERCONDUCTORS: NBS CONTRIBUTION TO THE VAMAS INTERLABORATORY COMPARISON

L. F. Goodrich and S. L. Bray

Published in Cryogenics

Volume 29, pp. 699-709, July 1989 


\title{
Critical current measurements of $\mathrm{Nb}_{3} \mathrm{Sn}$ superconductors: NBS contribution to the VAMAS Interlaboratory Comparison*
}

\author{
L.F. Goodrich and S.L. Bray \\ Center for Electronics and Electrical Engineering, National Bureau of Standards, Boulder, \\ Colorado 80303, USA
}

\author{
Received 7 July 1988
}

\begin{abstract}
Critical current measurements on several $\mathrm{Nb}_{3} \mathrm{Sn}$ superconductors were made as part of an interlaboratory comparison (round robin). These measurements were made in conjunction with twenty-four laboratories from the European Economic Community, Japan and the USA as part of the Versailles Agreement on Advanced Materials and Standards (VAMAS). The results of the NBS measurements, including the effect of sample mounting techniques on the measured critical current, are given. A systematic study of the effect of measurement mandrel (tubular sample-holder made from G10 fibreglass-epoxy composite) geometry revealed that a seemingly small change in that geometry can result in a $40 \%$ change in the measured critical current at a magnetic field of $12 \mathrm{~T}$. Specifically, the radial thermal contraction of the measurement mandrel depends on its wall thickness and, thus, so does the conductor prestrain (at $4 \mathrm{~K}$ ) and, ultimately, the measured critical current. Techniques for reducing variation in the measured critical current are suggested.
\end{abstract}

Keywords: critical currents; superconductors; $\mathrm{Nb}_{3} \mathrm{Sn}$; VAMAS; NBS

\begin{abstract}
The measurements reported here represent the NBS contribution to a collaborative effort involving twentyfour laboratories from the European Economic Community, Japan and the USA under the Versailles Agreement on Advanced Materials and Standards (VAMAS). The purpose of this effort is to develop critical current $\left(I_{c}\right)$ measurement techniques for $\mathrm{Nb}_{3} \mathrm{Sn}$ superconductors that yield consistent results at different laboratories. These were part of an interlaboratory comparison (round robin) where each of the participating laboratories was supplied with samples of three different conductors that had been reacted in various coil geometries to match the $I_{c}$ measuring instruments of the various laboratories. Each laboratory then measured the $I_{c}$ of the three samples at several applied magnetic field strengths and two orientations (parallel and antiparallel to the sample coils' self field). Preliminary comparisons of the laboratories' results showed a large variation in the measured critical current. In response, the NBS study was expanded to address specific measurement variables that might have resulted in these discrepancies. The analytical benefit of round robin collaborations is evidenced by these results where the interlaboratory comparison of data demonstrated that a subtle measurement variable can result in a large difference in the measured $I_{\mathrm{c}}$.
\end{abstract}

* Publication of the National Bureau of Standards, not subject to copyright
General considerations for accurate critical current measurements have been given ${ }^{1,2}$. The predominant variables that affect critical current measurements of $\mathrm{Nb}_{3} \mathrm{Sn}$ wires fall into four categories: sample reaction conditions, measurement mandrel (sample holder) material and geometry, the method used for bonding the sample to its mandrel and damage incurred during shipping or in transferring the sample from the reaction mandrel (tubular stainless steel sample holder) to the measurement mandrel. In the latter three categories, it is the sensitivity of $\mathrm{Nb}_{3} \mathrm{Sn}$ to mechanical strain that results in variations in the measured $I_{c}^{3}$. For these measurements, like samples were reacted at a central location under presumably the same conditions; consequently, the reaction variables were not suspected of being a significant factor. However, in order to address the question of shipping damage, specimens of each sample were reacted and measured at NBS; thus, some measure of the conductor's sensitivity to reaction conditions was obtained. The measurement mandrel material and geometry can work in conjunction with the sample bonding technique to create a dominant measurement variable, the axial strain of the conductor. Under a condition where 1 , the circumferential thermal contraction of the measurement mandrel is greater than the axial thermal contraction of the conductor, and 2 the bonding method creates a strong coupling between the sample and the measurement mandrel, significant axial strain of the conductor at $4 \mathrm{~K}$ may result. It was discovered in this study that the 
circumferential thermal contraction of the G10 fibreglass-epoxy tubes used for the measurement mandrels in this study depends on the tube's geometry, owing to its composite structure. In particular, the ratio of the tube's wall thickness to its radius strongly affects its thermal contraction. Based on these observations, a systematic study of the effect of G10 measurement mandrel geometry on the measured critical current was made.

For the measurement mandrel study, a filled epoxy adhesive was used for bonding the sample to the mandrel. This technique ensured the strong bond and rigid coupling between the sample and mandrel that is necessary for positive stress transmission. In one case, varnish was used as the sample mounting adhesive to study the effect of the Lorentz force on the critical current of a weakly constrained sample. Another variable that ciepends on the bonding technique is the thermal coupling between the sample and the liquid helium bath. Although a complete epoxy coating of the sample results in uniform mechanical constraint, it also presents a thermal impedance that in some cases may cause a lower measured critical current.

The purpose of the measurements presented here is to document and better understand the sources of inconsistencies in the measured critical current of $\mathrm{Nb}_{3} \mathrm{Sn}$ conductors. This understanding is ultimately necessary for determining a consistent and practical unified measurement method. In theory, a measurement method that would give consistent results is easily achieved by imposing extensive constraints on all aspects of the measurement; however, this approach is impractical considering the variety of $I_{\mathrm{c}}$ measurement systems that are presently in use. Consequently, a practical measurement method should impose the least number of constraints that yield acceptable results.

More challenging than the issue of consistent measurements is that of correct measurements. The basic problem that these round robin tests reveal is that the correct and/or best method for critical current measurements of $\mathrm{Nb}_{3} \mathrm{Sn}$ is unclear. Thus, a statement regarding the accuracy of these measurements is currently inappropriate. A future joint publication on the results from all participants is planned.

\section{Samples}

In order to avoid the identification of commercial products by the manufacturer's name, the samples measured in this round robin will be identified by letter only. Some physical data are provided to indicate the general type of conductor used in these tests.

Sample $X$ had a wire diameter of $0.68 \mathrm{~mm}$ and 37 sub-bundles each containing $150 \mathrm{Nb}$ filaments. The conductor fabrication method was an internal tin diffusion process. A single diffusion barrier of Ta separates the filament region from the outer layer of $\mathrm{Cu}$. The sample reaction temperature was $700^{\circ} \mathrm{C}$ for $48 \mathrm{~h}$.

Sample $Y$ had a wire diameter of $1.0 \mathrm{~mm}$ and 7 sub-bundles each containing $721 \mathrm{Nb}$ filaments. The conductor fabrication method was a bronze diffusion process. Each sub-bundle had a Nb diffusion barrier and the matrix was a CuSnTi alloy. The sample reaction temperature was $670^{\circ} \mathrm{C}$ for $200 \mathrm{~h}$.

Sample $\mathrm{Z}$ had a wire diameter of $0.80 \mathrm{~mm}$ and 114 sub-bundles each containing $54 \mathrm{NbTa}$ filaments in a bronze matrix. The conductor fabrication method was a bronze diffusion process. The $\mathrm{Cu}$ stabilizer was located at the centre of the wire separated by a Ta barrier. The sample reaction temperature was $700^{\circ} \mathrm{C}$ for $96 \mathrm{~h}$.

Specimens of each sample were heat treated (reacted) at their central laboratory (central reaction). Other specimens of each sample were to be reacted at each individual measurement laboratory (self reaction). A comparison of results from these two groups gives an indication of reaction and shipping variables. Each specimen was reacted on a stainless steel tube that had a spiral groove on it to define the coil pitch. The surface of the stainless steel had been oxidized to reduce the chance of diffusion bonding between the sample and the reaction mandrel. When bonding does occur, the sample can be damaged when it is removed for mounting onto the measurement mandrel. The self reactions performed at NBS were done in a three-zone, vacuum tube furnace. The temperature of the furnace was controlled during the reaction to a precision of $\pm 5^{\circ} \mathrm{C}$. The furnace temperature calibration was checked and was accurate to $\pm 10^{\circ} \mathrm{C}$.

\section{Experimental details}

The measurement mandrels were constructed from a G10 tube that had a cylindrical copper current contact rigidly attached to each end. Each contact had a superconductive bus bar ( $\mathrm{Cu}$ and $\mathrm{Nb}_{3} \mathrm{Sn}$ tape) connected to it. The sample was transferred from the reaction mandrel onto the measurement mandrel by carefully unthreading the sample from the reaction mandrel and slipping it on to the measurement mandrel (not grooved). The one current contact that was removed for this transfer was then reattached to the measurement mandrel and the sample was tightened onto the mandrel. Each end of the sample was then soldered onto its current contact. An effort was made to avoid a complete superconductive loop formed by the sample and bus bar around the current contact. This was done in order to a void persistent currents. Three pairs of adjacent voltage taps were placed along the centre of the sample. Each pair had a separation of about $10 \mathrm{~cm}$ and there was a $1-2 \mathrm{~cm}$ gap between adjacent pairs. The sample was bonded to the mandrel with filled epoxy (or varnish in one case) and allowed to cure. The epoxy coat was kept thin to enhance the thermal conductivity from the sample to the helium bath. This unit was then mounted onto the test fixture where the electrical connections were completed.

The measurements were made at about $4.2 \mathrm{~K}$ in a $12 \mathrm{~T}$, $12.7 \mathrm{~cm}$ diameter superconductive solenoidal magnet, which has a homogeneity of $\pm 0.2 \%$ over the region spanned by the voltage taps. Since the ambient atmospheric pressure at this test site is low, resulting in an equilibrium liquid helium temperature of $4.0 \mathrm{~K}$, the dewar pressure was elevated. The pressure in the helium dewar was controlled with a diaphragm-type manostat and the temperature was deduced from the measured pressure. A heater on the bottom of the helium dewar was used to remove the natural stratification and bring the bath into equilibrium at the elevated pressure.

The voltage-current curves for all three voltage tap pairs were recorded simultaneously with a digital processing uscilloscope. An analogue nanovoltmeter with an 
amplified output was used to measure the sample voitage of each tap pair. A battery powered current supply was used for the sample current ${ }^{4}$. The sample voltage was measured at numerous sample currents. These points along the voltage-current curve were analysed to determine $I_{\mathrm{c}^{\circ}}$ The measurements were made with forward (antiparallel magnetic fields, Lorentz force in) and reverse (parallel magnetic fields, Lorentz force out) current directions to determine the effect of self-field and sample-tomandrel bonding (strain effect). Unless otherwise stated, the $I_{\mathrm{c}}$ values given are averages over all taps, observations and current directions at an electric field strength criterion of $10 \mu \mathrm{V} \mathrm{m}{ }^{-1}$. The precision of the $I_{c}$ measurement was about $\pm 0.2 \%$.

Another parameter that was determined during the $I_{\mathrm{c}}$ measurement is $n$. The parameter $n$ is defined by the approximate voltage-current relationship,

$V=V_{\mathrm{o}}\left(I / I_{\mathrm{o}}\right)^{n}$

where $I_{0}$ is a reference critical current at a voltage criterion $V_{0}, V$ is the sample voltage, $I$ is the sample current, and $n$ reflects the shape of the curve with typical values from 20 to 60 (a higher number means a sharper transition). A lower value of $n$ can be an indication of sample damage or strain.

Some specimens were difficult to measure because they would quench (thermal runaway into the normal state) at relatively low sample voltages (electric field strength about $10 \mu \mathrm{V} \mathrm{m}^{-1}$ ). This resulted in some variation in the $I_{\mathrm{c}}$ values due to the small voltage range that could be analysed. In some cases this low quench level was traced to damaged segments (weak links) adjacent to a current contact. These weak links may have been due to sample damage introduced in removing the sample from the reaction mandrel, transferring the sample to the measurement mandrel, or by strain in the transition length near the current contact. The transition between the contact and the G10 has two intrinsic problems. One problem arises from the differential thermal contraction between the contact and the G10, which causes a stress concentration. The other problem is the differential contraction between the copper contact and the sample, which introduces additional sample pre-stain. Difficulties in measuring the $\mathrm{Z}$ sample may have been due to its high current density $\left(535 \mathrm{~A} \mathrm{~mm}^{-2}\right.$ at $\left.10 \mathrm{~T}\right)$ and bronze surface layer (larger contact resistance) causing sample heating.

\section{Results}

\section{Sample $X$}

A summary of the data on sample $\mathrm{X}$ is given in Table 1 and Figures 1-4. The information contained in Table 1 is the critical current data, sample mandrel dimensions and intercomparisons of critical current data. The critical current data are given at magnetic fields from 6 to $12 \mathrm{~T}$. The effect of sample mandrel geometry, bonding method, thermal cycle, and central or self reaction variables were studied. Table 1 contains key words that relate to these variables. Each column, which is labelled with a letter from $A$ to $G$, is a data set taken for a single thermal cycle. Specimens are numbered from 1 to 4 . Different data columns that are for the same specimens (columns A and $B$ for example) indicate a thermal cycle where the specimen was measured at $4 \mathrm{~K}$, warmed to room temperature, cooled again to $4 \mathrm{~K}$ and remeasured. In some

Table 1 Data on sample $X$; critical current for sample $X$

\begin{tabular}{|c|c|c|c|c|c|c|c|}
\hline Specimen & 1 & 1 & 1 & 2 & 3 & 3 & 4 \\
\hline$\mu_{0} H(T)$ & $\begin{array}{c}\text { Central } \\
\text { thick } \\
\text { (A) }\end{array}$ & $\begin{array}{c}\text { Central } \\
\text { thick } \\
\text { (B) }\end{array}$ & $\begin{array}{l}\text { Central } \\
\text { thin } \\
\text { bored } \\
\text { (C) }\end{array}$ & $\begin{array}{l}\text { Central } \\
\text { thin } \\
\text { (D) }\end{array}$ & $\begin{array}{l}\text { Self } \\
\text { thin } \\
\text { spiral } \\
\text { (E) }\end{array}$ & $\begin{array}{l}\text { Self } \\
\text { thin } \\
\text { (F) }\end{array}$ & $\begin{array}{l}\text { Self } \\
\text { thin } \\
\text { varnish } \\
\text { (G) }\end{array}$ \\
\hline 6 & 343.3 & 343.2 & 397.3 & 386.3 & 388.9 & 386.6 & 374.5 \\
\hline 7 & 268.4 & 267.6 & 318.4 & 308.9 & 312.4 & 310.3 & 297.9 \\
\hline 8 & 207.0 & 207.4 & 253.8 & 246.0 & 249.7 & 247.6 & 235.9 \\
\hline 9 & 156.5 & 156.6 & 200.1 & 193.5 & 197.4 & 195.6 & 184.8 \\
\hline 10 & 113.6 & 194.0 & 154.7 & 149.3 & 153.2 & 151.6 & 142.1 \\
\hline 11 & & 78.5 & 116.0 & 111.9 & 114.6 & 112.8 & 106.6 \\
\hline 12 & & 50.2 & 83.5 & 80.4 & 82.5 & 81.1 & 74.6 \\
\hline \multicolumn{8}{|c|}{ Sample mandrel dimensions } \\
\hline $\begin{array}{l}\text { Wall }(\mathrm{mm}) \\
\text { Rad. }(\mathrm{mm}) \\
w / r\end{array}$ & $\begin{array}{r}9.3 \\
15.6 \\
60 \%\end{array}$ & $\begin{array}{r}9.3 \\
15.6 \\
60 \%\end{array}$ & $\begin{array}{r}2.5 \\
15.6 \\
16 \%\end{array}$ & $\begin{array}{r}2.0 \\
15.6 \\
13 \%\end{array}$ & $\begin{array}{r}1.5 \\
15.6 \\
10 \%\end{array}$ & $\begin{array}{r}1.5 \\
15.6 \\
10 \%\end{array}$ & $\begin{array}{r}2.0 \\
15.6 \\
13 \%\end{array}$ \\
\hline \multicolumn{8}{|c|}{ Intercomparison of critical current data } \\
\hline$\mu_{0} H(T)$ & $\begin{array}{c}\text { Delta } \\
(A-B) / B\end{array}$ & $\begin{array}{c}\text { Delta } \\
(B-C) / C\end{array}$ & $\begin{array}{c}\text { Delta } \\
(C-D) / D\end{array}$ & & $\begin{array}{c}\text { Delta } \\
(E-D) / D\end{array}$ & $\begin{array}{c}\text { Delta } \\
(F-D) / D\end{array}$ & $\begin{array}{c}\text { Delta } \\
(G-D) / D\end{array}$ \\
\hline $\begin{array}{r}6 \\
7 \\
8 \\
9 \\
10 \\
11 \\
12\end{array}$ & $\begin{array}{r}0.0 \% \\
0.3 \% \\
-0.2 \% \\
-0.1 \% \\
-0.4 \%\end{array}$ & $\begin{array}{l}-13.6 \% \\
-16.0 \% \\
-18.3 \% \\
-21.7 \% \\
-26.3 \% \\
-32.3 \% \\
-39.9 \%\end{array}$ & $\begin{array}{l}2.8 \% \\
3.1 \% \\
3.2 \% \\
3.4 \% \\
3.6 \% \\
3.7 \% \\
3.9 \%\end{array}$ & & $\begin{array}{l}0.7 \% \\
1.1 \% \\
1.5 \% \\
2.0 \% \\
2.6 \% \\
2.4 \% \\
2.6 \%\end{array}$ & $\begin{array}{l}0.1 \% \\
0.5 \% \\
0.7 \% \\
1.1 \% \\
1.5 \% \\
0.8 \% \\
0.9 \%\end{array}$ & $\begin{array}{l}-3.1 \% \\
-3.6 \% \\
-4.1 \% \\
-4.5 \% \\
-4.8 \% \\
-4.7 \% \\
-7.2 \%\end{array}$ \\
\hline
\end{tabular}




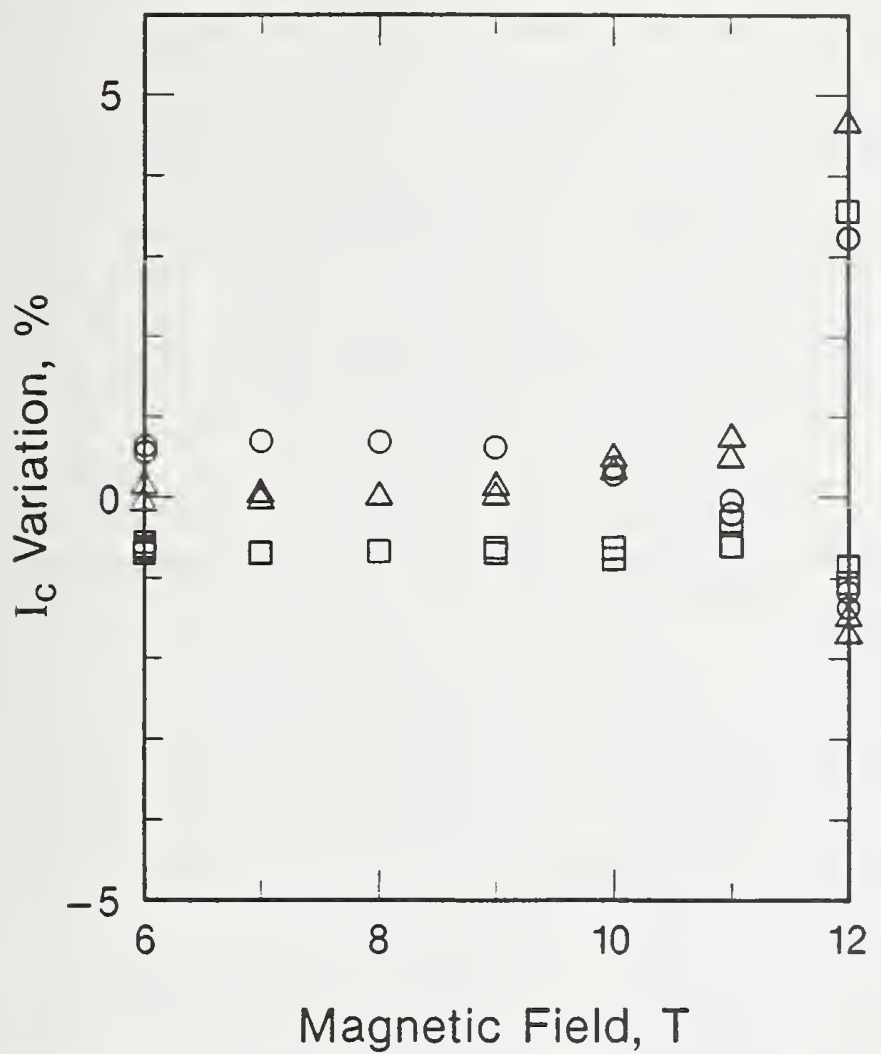

Figure 1 Percentage variation of $I_{\mathrm{c}}$ about the average $I_{\mathrm{c}}$ versus magnetic field for specimen $G$ (self, thin, varnish) of sample $X$. Tap symbols: 0,$1 ; \square, 2 ; \triangle, 3$

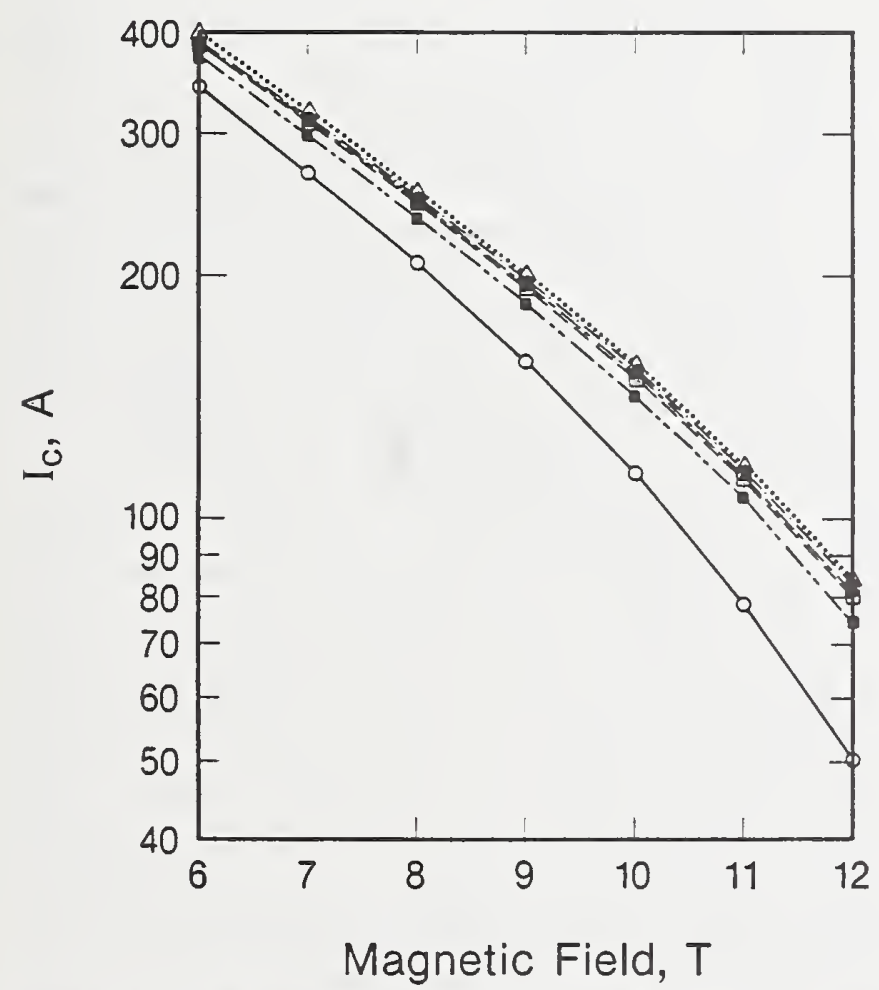

Figure 2 A semilogarithmic plot of $I_{c}$ at $10 \mu \mathrm{V} \mathrm{m} \mathrm{m}^{-1}$ versus magnetic field for specimens of sample X. $E_{c}=10 \mu \mathrm{Vm}^{-1}$. $O-O$. Central thick; $\triangle \cdots \triangle$, central bored; $\square---\square$, central thin: $-\cdot-$ self thin spiral; $\triangle \ldots-\ldots$, self thin; $\square-(-\square$, self thin varnish

cases, the mounting configuration of the specimen was changed between these measurements (columns B and $\mathrm{C}$ for example). The sequence of the thermal cycles was from left to right in the table.

The key words heading the columns of Table I (central, thick, thin, etc.) are used to indicate the measurement variables. Central indicates reaction of the specimen at

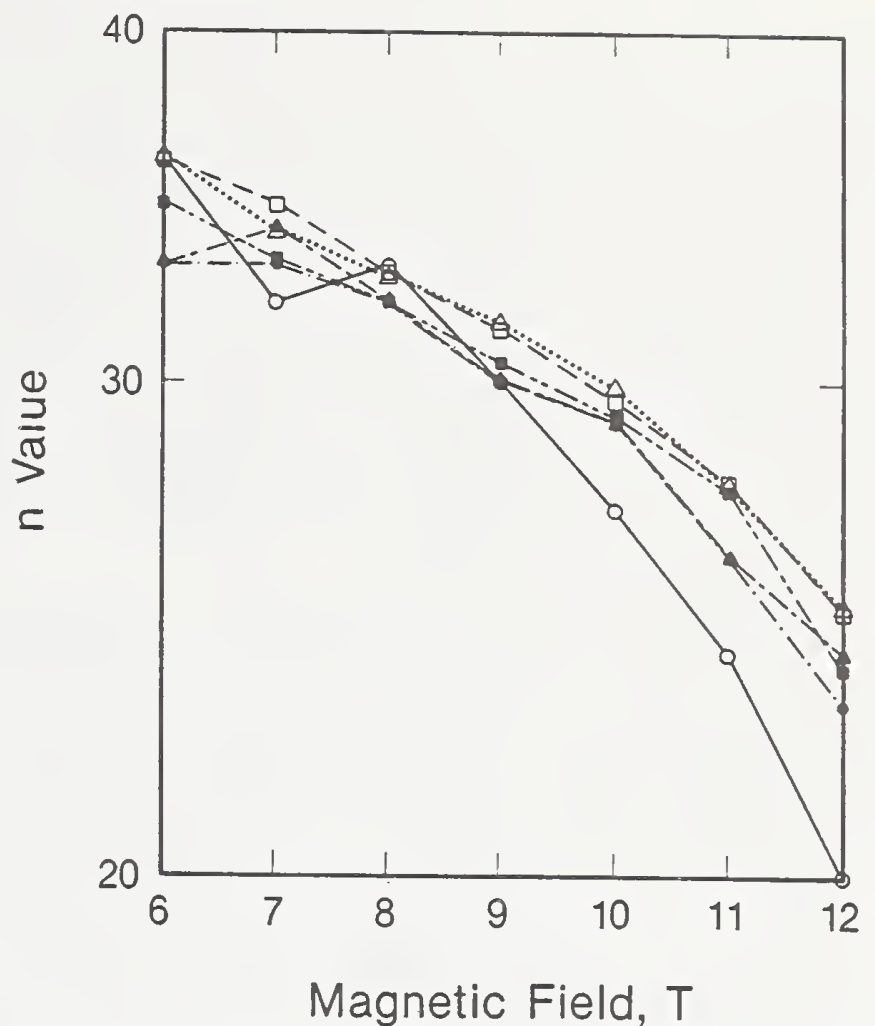

Figure 3 A semilogarithmic plot of $n$ value versus magnetic field for specimens of sample $X . \bigcirc-O$, Central thick: $\triangle \cdots \triangle$, central bored; $\square---\square$, central thin; $-\cdot-0$, self thin spiral; $\Delta---\Delta$. self thin; 1

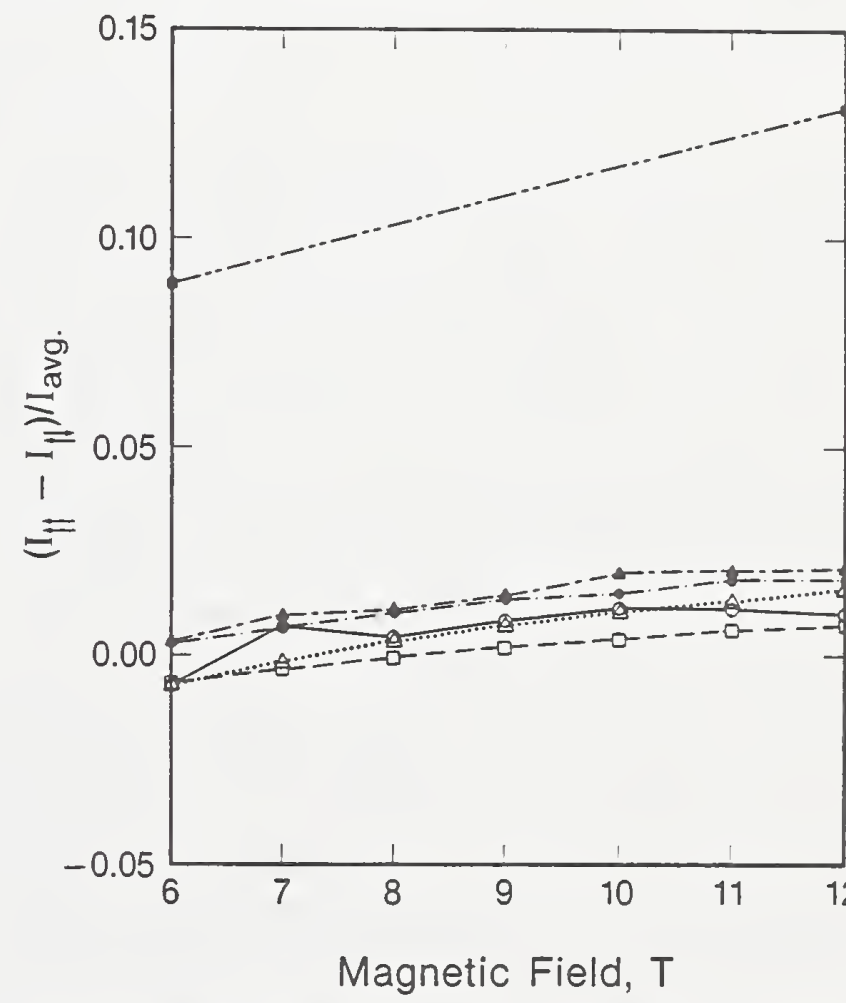

Figure 4 Percentage difference between $/ c$ measured with reverse and forward current directions versus magnetic field for specimens of sample $X . E_{\mathrm{c}}=10 \mu \mathrm{V} \mathrm{m}^{-1} .0-0$. Central thick; $\triangle \cdots \Delta$, central bored: $\square--\square$. central thin; $-\cdot-\bullet$. self thin spiral; $\Delta---\Delta$. self thin; $\square-(-\square$, self thin varnish

a central laboratory and self indicates reaction at the individual measurement laboratory. Thick and thin refer to the ratio of wall thickness to outer radius of the sample mandrel. Under the Sample Mandrel Dimensions section of the table, these specific values are listed for each data set. Bored refers to a specimen that was originally mounted and measured on a thick-walled mandrel. The 
specimen was then brought to room temperature (thermally cycled) and the mandrel inner diameter was bored to a larger dimension, resulting in a reduced wall thickness. The specimen's $I_{\mathrm{c}}$ was then remeasured. The typical method for bonding samples to measurement mandrels was to coat the sample and the entire surface of the mandrel with epoxy. Two variations of this technique are indicated by spiral and varnish. Spiral indicates that the sample was bonded to the mandrel with a thin stripe of epoxy along its length. Varnish indicates that the sample and the mandrel were completely coated with varnish rather than epoxy.

The centrally reacted specimens were numbers 1 and 2. A comparison of the $I_{c}$ data in columns $\mathrm{A}$ and $\mathrm{B}$ is a measure of the $I_{c}$ variation due to thermal cycling. The maximum variation was $0.4 \%$ at $10 \mathrm{~T}$ (the highest magnetic field for column $\mathrm{A}$ ) as shown in the intercomparison portion of the table $([\mathrm{A}-\mathrm{B}] / \mathrm{B})$. This small variation upon thermal cycling was representative of all observations. Column $C$ was a special case of a thin-walled mandrel in that it was bored (machined with the specimen mounted on the mandrel). Because this was the same specimen as $\mathrm{A}$ and $\mathrm{B}$, the comparison is a systematic one. The intent of this procedure was to determine the effect of mandrel wall thickness on the measured $I_{c}$ of a particular specimen. The intercomparison data ([BC]/C) shows a strongly field dependent variation of $I_{\mathrm{c}}$ (indicative of a strain effect) with a maximum difference of $39 \%$ at 12 T. This is believed to result from a change in the conductor pre-strain (at $4 \mathrm{~K}$ ) due to variation in thermal contraction between thick and thin walled G-10 tubes. An approximate measure of the thermal contraction (from room to liquid nitrogen temperature) variations indicated a $0.2 \%$ greater contraction for the thickwalled tube. Strain sensitivity measurements on this sample indicate that this additional pre-strain would result in an $I_{c}$ degradation in the order of $33 \%$. The composite nature of $\mathrm{G}-10$ plate $^{5-7}$ in conjunction with the compound geometry of a tube may result in a variation in radial thermal contraction between tubes of different wall thickness. Machining may have changed the mandrel's outer diameter at room temperature due to stress relief and, thus, the pre-strain state of the conductor. More details regarding thermal contraction of G-10 measurement mandrels and the resulting critical current degradation due to axial strain are presented below. The rest of the intercomparisons in Table 1 were made relative to $\mathrm{D}$ (central, thin) since it was thought to be a more ideal baseline for comparisons. The comparison between $C$ and $D$ showed only a small difference, $3-4 \%$, with very little field dependence $([\mathrm{C}-\mathrm{D}] / \mathrm{D})$. This indicates that the effect of boring was about equivalent to that of simply mounting a specimen on a thin-walled tube.

The self reacted specimens on thin wall tubes were numbers $3(E$ and $F$ ) and $4(G)$. For data set $E$ the specimen was bonded to the mandrel with a thin stripe of epoxy along its length (spiral). This spiral mounting technique was employed to examine the effect of a less rigid containment structure. The comparison between $\mathrm{D}$ and $\mathrm{E}$ was within $2.6 \%$. Furthermore, the comparison of $F$ ( $E$ with a continuous epoxy coat) with $D$ was indicative of the small difference, $1.5 \%$, between central and self reacted wires. The systematic difference between $\mathrm{E}$ and F was, thus, $1.7 \%$ or less. This demonstrates that a continuous epoxy coat was not very detrimental in this case. Specimen $4(\mathrm{G})$ was bonded to the mandrel with a coat of varnish in order to investigate the bonding properties of a weaker and less permanent adhesive. The $I_{\mathrm{c}}$ values in column $\mathrm{G}$ were averages of data taken with forward current only (Lorentz force into the mandrel). Some measurements were made with reverse current (Lorentz force away from the mandrel) at 6 and $12 \mathrm{~T}$ but these results were not included in this table. For this data set, the $12 \mathrm{~T}$ (magnetic field of lowest Lorentz force) measurements were made first to reduce the possibility of irreversible strain damage. However, even at this relatively low Lorentz force, there was an irreversible shift in the forward $I_{\mathrm{c}}$ after the reverse $I_{\mathrm{c}}$ measurements were made.

Figure $l$ is a plot of the percentage difference between the observed forward $I_{\mathrm{c}} \mathrm{s}$ and the $I_{\mathrm{c}}$ a veraged over all taps and observations at each magnetic field. For the data between 6 and $11 \mathrm{~T}$, the variation of measured $I_{\mathrm{c}} \mathrm{s}$ among the pairs of voltage taps was typical of specimen homogeneity. The indicated precision of the repeat determinations (indicated by multiple data points for the same tap at the same field) was also typical of these measurements. The lower group of data points at $12 \mathrm{~T}$ were the first measurements of this specimen and were made with forward current (Lorentz force in). The upper group of data points at $12 \mathrm{~T}$ were taken after reverse $I_{\mathrm{c}}$ (Lorentz force out) measurements were made (the data for reverse $I_{\mathrm{c}}$ is not shown on the plot). These $12 \mathrm{~T}$ data indicate an irreversible enhancement in the forward $I_{c}$ which can also be seen in the $(\mathrm{G}-\mathrm{D}) / \mathrm{D}$ column of Table 1 where a discontinuity exists between 11 and $12 \mathrm{~T}$. The limitations of varnish as a bonding agent are demonstrated by these results. First, the limited tensile strength of the varnish is incapable of constraining the wire on the mandrel even under moderate, outward Lorentz force. Second, even under compressive loads, the varnish seems incapable of reproducing the results obtained with epoxy. Sample inhomogeneity could also explain this difference.

Figure 2 is a semi-logarithmic plot of $I_{\mathrm{c}}$ versus magnetic field. A semi-logarithmic plot was selected because it illustrates the percentage differences between the curves. The central, thick curve is significantly lower than the others and the thin, varnish curve is slightly lower. Figure 3 is a semi-logarithmic plot of $n$ versus magnetic field. The significant element of this plot is the lower values of $n$ for the central, thick curve at the higher magnetic fields. This is consistent with a strain degradation. The magnitude of the differences among the other curves is insignificant for this measurement variable. Figure 4 is a plot of the percentage difference between reverse and forward $I_{c}$ versus magnetic field. The thin, varnish curve illustrates the large strain effect, $13 \%$ at $12 \mathrm{~T}$, when the sample is not adequately constrained to the mandrel. The smaller difference at $6 \mathrm{~T}$ was probably due to the decreased strain sensitivity at the lower magnetic field. The other curves illustrate the competition between the self-field effect, which is significant at the lower magnetic fields, and the strain effect, which is dominant at the higher magnetic fields. The maximum difference for the specimens bonded with epoxy was only $2 \%$ at $12 \mathrm{~T}$ which indicates that this bonding method is very positive.

\section{Sample Y}

A summary of the data on sample $\mathrm{Y}$ is given in Table 2 and Figures 5-9. The centrally reacted specimens were 
Critical current measurements of $\mathrm{Nb}_{3} \mathrm{Sn}$ superconductors: L.F. Goodrich and S.L. Bray

Table 2 Data on sample $Y$

\begin{tabular}{|c|c|c|c|c|c|c|}
\hline \multicolumn{7}{|c|}{ Critical current (amperes) at $10 \mu \mathrm{V} \mathrm{m}^{-1}$} \\
\hline \multirow[t]{2}{*}{ Specimen } & 1 & 2 & 3 & 3 & 4 & 4 \\
\hline & $\begin{array}{l}\text { Central } \\
\text { thick }\end{array}$ & $\begin{array}{l}\text { Central } \\
\text { thick }\end{array}$ & $\begin{array}{l}\text { Central } \\
\text { thick }\end{array}$ & $\begin{array}{c}\text { Central } \\
\text { thin } \\
\text { bored }\end{array}$ & $\begin{array}{l}\text { Self } \\
\text { thin }\end{array}$ & $\begin{array}{l}\text { Self } \\
\text { thin }\end{array}$ \\
\hline$\mu_{0} H(T)$ & (A) & (B) & (C) & (D) & $(E)$ & $(F)$ \\
\hline 6 & 398.1 & 372.5 & 397.1 & 447.8 & 429.1 & \\
\hline 7 & 332.7 & 311.0 & 333.1 & 377.6 & 359.2 & \\
\hline 8 & 279.4 & 261.2 & 280.0 & 320.5 & 302.4 & \\
\hline 9 & 235.0 & 220.0 & 235.3 & 272.9 & 255.7 & \\
\hline 10 & & 184.9 & 198.9 & 232.7 & 216.1 & \\
\hline 11 & & 154.8 & 166.9 & 198.3 & 182.1 & 182.9 \\
\hline 12 & & 128.6 & 139.1 & 168.1 & & 153.4 \\
\hline \multicolumn{7}{|c|}{ Sample mandrel dimensions } \\
\hline & (A) & (B) & (C) & (D) & $(E)$ & $(F)$ \\
\hline Wall $(\mathrm{mm})$ & 9.3 & 9.3 & 9.3 & 2.5 & 2.0 & 2.0 \\
\hline Rad. (mm) & 15.6 & 15.6 & 15.6 & 15.6 & 15.6 & 15.6 \\
\hline ratio & $60 \%$ & $60 \%$ & $60 \%$ & $16 \%$ & $13 \%$ & $13 \%$ \\
\hline \multicolumn{7}{|c|}{ Intercomparison of critical current data } \\
\hline$\mu_{0} H(T)$ & $(\mathrm{A}-\mathrm{C}) / \mathrm{C}$ & $(\mathrm{B}-\mathrm{C}) / \mathrm{C}$ & $(C-D) / D$ & & $(E-D) / D$ & $(F-D) / D$ \\
\hline 6 & $0.3 \%$ & $-6.2 \%$ & $-11.3 \%$ & & $-4.2 \%$ & \\
\hline 7 & $-0.1 \%$ & $-6.6 \%$ & $-11.8 \%$ & & $-4.9 \%$ & \\
\hline 8 & $-0.2 \%$ & $-6.7 \%$ & $-12.6 \%$ & & $-5.6 \%$ & \\
\hline 9 & $-0.1 \%$ & $-6.5 \%$ & $-13.8 \%$ & & $-6.3 \%$ & \\
\hline 10 & & $-7.0 \%$ & $-14.5 \%$ & & $-7.1 \%$ & \\
\hline 11 & & $-7.2 \%$ & $-15.8 \%$ & & $-8.2 \%$ & $-7.8 \%$ \\
\hline 12 & & $-7.5 \%$ & $-17.3 \%$ & & & $-8.7 \%$ \\
\hline
\end{tabular}

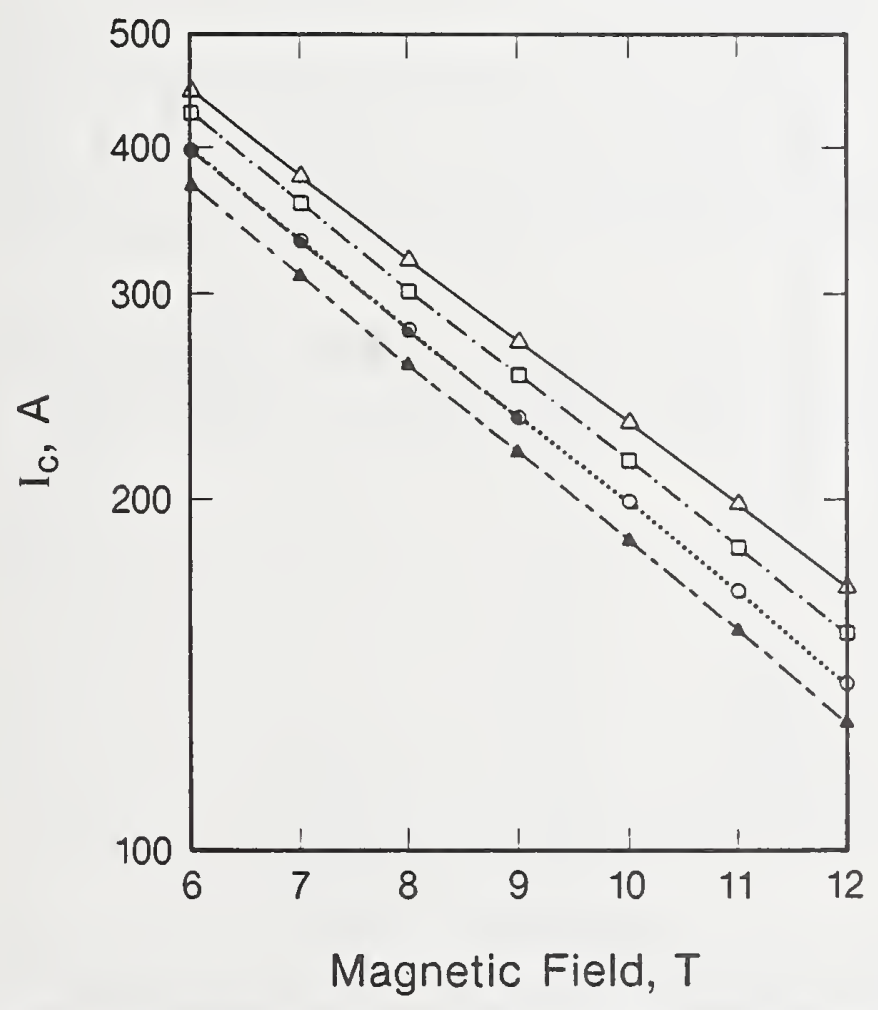

Figure 5 Semilogarithmic plot of $I_{c}$ at $10 \mu \mathrm{V} \mathrm{m}^{-1}$ versus magnetic field for specimens of sample $Y . E_{\mathrm{c}}=10 \mu \mathrm{V} \mathrm{m}^{-1} .0 \cdots O$. Central thick $C: \triangle-\triangle$, central bored: $\square-\cdot-\square$, self thin;.--0 . central thick $A ; \Delta-\cdots \Delta$, central thick $B$

numbers 1, 2 and 3. With respect to mandrel geometry, mounting technique and the reaction site, these specimens were the same. Data sets $\mathrm{A}$ and $\mathrm{C}$ are in good agreement $(0.3 \%$ maximum variation $)$; however, set $B$ is in contradiction with both $\mathrm{A}$ and $\mathrm{C}(7.5 \%$ maximum difference).
There are several possible explanations for this discrepancy including shipping damage, mounting damage, sample inhomogeneity and variation in mandrel thermal contraction. These data are insufficient to determine the most likely source of this effect. Data set $D$ was acquired on specimen 3 after boring and again is an indication of the pre-strain effect. The magnitude of this $I_{\mathrm{c}}$ enhancement was $17 \%$ at $12 \mathrm{~T}$ and is in approximate agreement with a $0.2 \%$ change in the pre-strain state of the wire as explained for sample X above. Sample Y's higher upper critical field would explain a lower strain sensitivity than that of sample $X(39 \%$ at $12 \mathrm{~T})$.

Data sets $E$ and $F$, self reacted specimen number 4 , are compared to data set $\mathrm{D}$ and again there is a similar $I_{\mathrm{c}}$ discrepancy $(8.7 \%$ at $12 \mathrm{~T})$ between two comparable specimens. The additional possible explanation for this is the fact that these specimens were reacted at different sites. A comparison of data sets $E$ and $F$ was another check on the effect of thermal cycling. These abbreviated data sets and resulting thermal cycles were the result of one of several magnet quenches and, as in the previous cases, the effect of thermal cycling on $I_{c}$ appears to be about $0.4 \%$.

The most significant result for this sample is, again, the dependence of $I_{c}$ on mandrel wall thickness.

Figure 5 is a semi-logarithmic plot of $I_{c}$ versus magnetic field. These curves show two data bands: an upper band, associated with the thin-walled specimens, and a lower band, associated with thick-walled specimens. While there is considerable variation in the curves, the dominant variation is between the thick and thin-walled mandrels. Figure 6 is a semi-logarithmic plot of $n$ versus magnetic field. The larger data scatter for the central thick $C$ curve may be due to the tap-to-tap $I_{c}$ variation observed on 


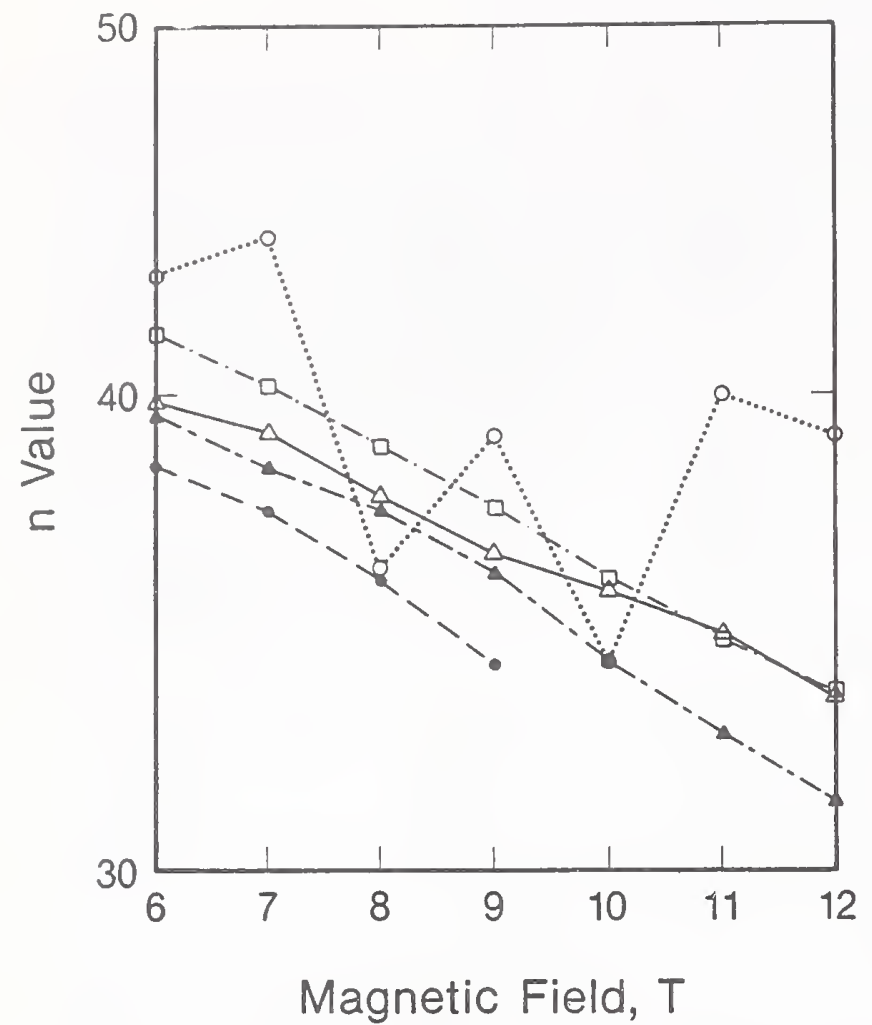

Figure 6 A semilogarithmic plot of $n$ value versus magnetic field for specimens of sample Y. $O \cdots O$. Central thick $C: \triangle-\triangle$. central bored; $\square-\cdot-\square$, self thin; ----0 , central thick $A$; $\Delta-\cdots \Delta$, central thick $B$

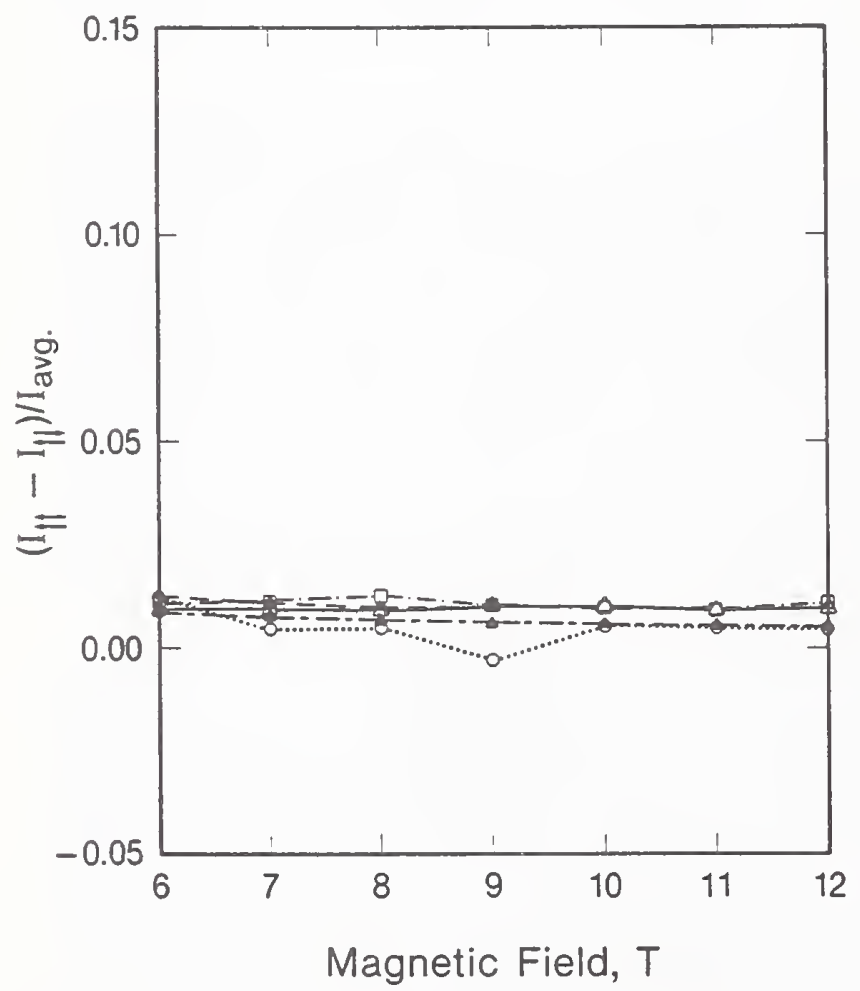

Figure 7 Percentage difference between $/ \mathrm{c}$ measured with reverse and forward current directions versus magnetic field for specimens of sample $Y, E_{c}=10 \mu \mathrm{Vm}^{-1} .0 \cdots O$. Central thick $\mathrm{C}: \triangle-\triangle$ central bored; $\square-\cdot-\square$; self thin; $-0 .-$, central thick $A$; $\Delta--\Delta$, central thick $B$

this specimen. The magnitude of the differences among the other curves is insignificant for this measurement variable. Figure 7 is a plot of the percentage difference between forward and reverse $I_{\mathrm{c}}$ versus magnetic field. In this case the maximum difference was only about $1 \%$. These curves are similar to those for sample X, Figure 4,

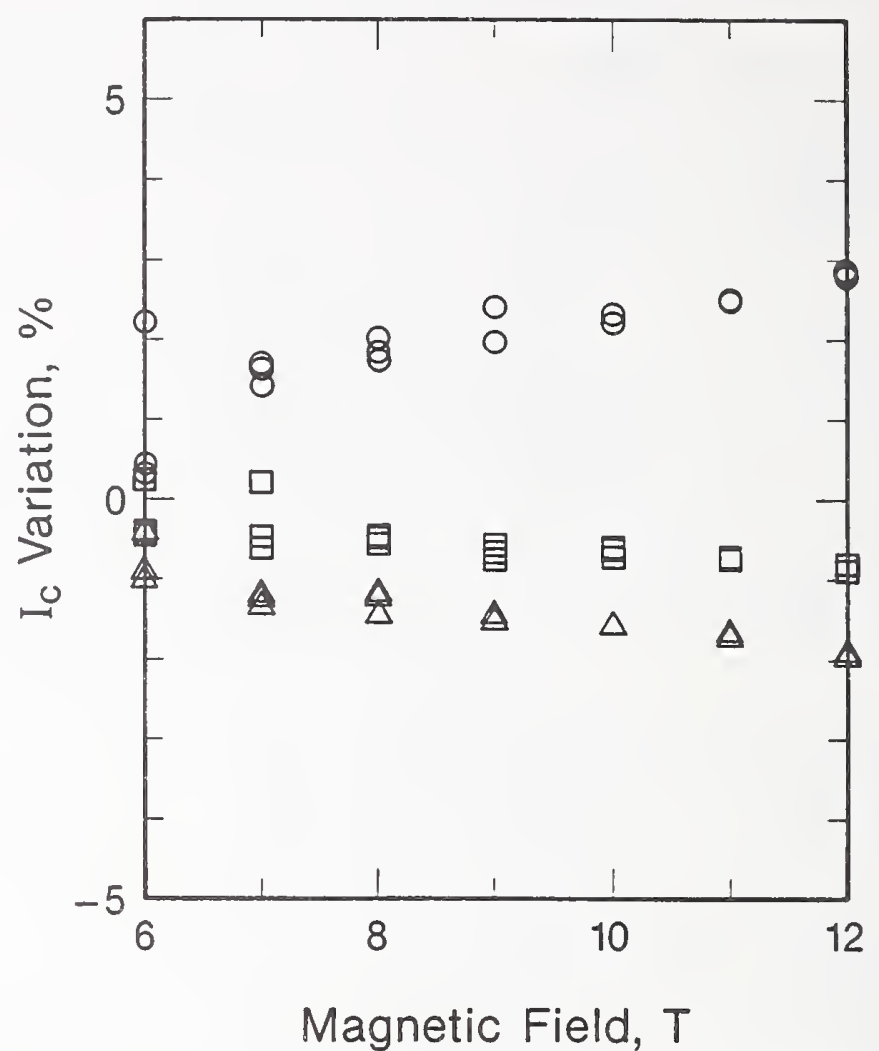

Figure 8 Percentage variation of $I_{\mathrm{c}}$ about the average $I_{\mathrm{c}}$ versus magnetic field for specimen $C$ (central, thick) of sample $Y$. Tap symbols: 0,$1 ; \square, 2 ; \triangle, 3$

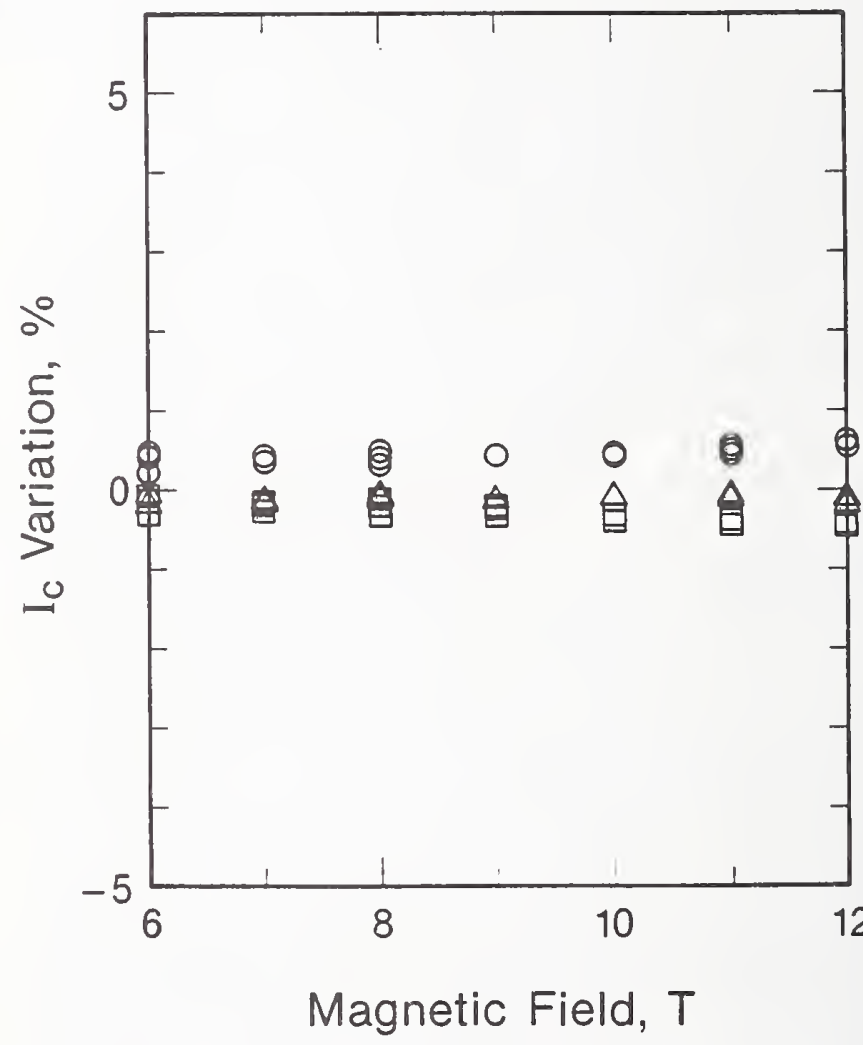

Figure 9 Percentage variation of $I_{c}$ about the average $I_{c}$ versus magnetic field for specimen $D$ (central, thick, bored) of sample $Y$. Tap symbols: 0,$1 ; \square, 2 ; \triangle, 3$

and indicate that the specimen was well bonded to the mandrel.

Figures 8 and 9 are plots of the percentage difference between the observed forward $I_{\mathrm{c}} \mathrm{s}$ and the $I_{\mathrm{c}}$ averaged over all taps and observations at each magnetic field. Both of these plots contain data for specimen 3 , the 
difference being that Figure 8 contains central thick $\mathrm{C}$ data and Figure 9 contains central thick-bored D data. The significant difference between the two plots is that the tap-to-tap $I_{\mathrm{c}}$ variation, observed in Figure 8, was not present after boring as shown in Figure 9. One possible explanation is a reduced longitudinal variation in G10 thermal contraction after boring. The variation of measured $I_{\mathrm{c}} \mathrm{s}$ for the other specimens was similar to that of Figure 9.

\section{Sample Z}

A summary of the data on sample $\mathrm{Z}$ is given in Table 3 and Figures 10-12. The centrally reacted specimens were numbers 1 and 2 with specimen 3 being self reacted. Data sets $A, B$, and $C$ are in good agreement, with a $1.6 \%$ variation in $I_{\mathrm{c}}$ at $12 \mathrm{~T}$. The centrally reacted specimens were measured on mandrels that had an outer diameter of about $19 \mathrm{~mm}$ rather than the typical $31 \mathrm{~mm}$ of all the other specimens. Prior to this study, this diameter was convenient for the anticipated measurements and not expected to be a significant variable. Given these limited data and this extra variable, no additional conclusion can be made regarding mandrel geometry and reaction site.

Figure 10 is a semi-logarithmic plot of $I_{c}$ versus magnetic field. Figure 11 is a semi-logarithmic plot of $n$ versus magnetic field. The significant information contained in this plot is the relatively high $n$ value for this conductor. Figure 12 is a plot of the percentage difference between forward and reverse $I_{\mathrm{c}}$ versus magnetic field. In this case the maximum difference was only about $4 \%$. With the possible exception of the self reacted specimen, these curves are similar to those for samples $\mathrm{X}$ and $\mathrm{Y}$, Figures 4 and 7, and indicate that the specimen was well bonded to the mandrel. Within the limits of the measure-

Table 3 Data on sample Z

\begin{tabular}{|c|c|c|c|}
\hline \multicolumn{4}{|c|}{ Critical current (amperes) at $10 \mu \mathrm{V} \mathrm{m}-1$} \\
\hline Specimen & 1 & 2 & 3 \\
\hline$\mu_{0} H(T)$ & $\begin{array}{l}\text { Central } \\
\text { (A) }\end{array}$ & $\begin{array}{c}\text { Central } \\
\text { (B) }\end{array}$ & $\begin{array}{l}\text { Self } \\
\text { (C) }\end{array}$ \\
\hline 6 & & 544.1 & \\
\hline 7 & & 454.5 & 443.1 \\
\hline 8 & & 381.4 & 372.2 \\
\hline 9 & & 320.6 & 311.5 \\
\hline 10 & 270.6 & 268.9 & 263.5 \\
\hline 11 & 226.4 & 224.3 & 221.1 \\
\hline 12 & 187.6 & 185.6 & 184.6 \\
\hline \multicolumn{4}{|c|}{ Sample mandrel dimensions } \\
\hline & (A) & (B) & (C) \\
\hline Wall $(\mathrm{mm})$ & 3.0 & 3.0 & 2.0 \\
\hline $\operatorname{Rad}(\mathrm{mm})$ & 9.3 & 9.3 & 15.6 \\
\hline ratio & $32 \%$ & $32 \%$ & $13 \%$ \\
\hline \multicolumn{4}{|c|}{ Intercomparison of critical current data } \\
\hline$\mu_{0} H(T)$ & $(A-B) / B$ & & $(C-B) / B$ \\
\hline 6 & & & \\
\hline 7 & & & $-2.5 \%$ \\
\hline 8 & & & $-2.4 \%$ \\
\hline 9 & & & $-2.8 \%$ \\
\hline 10 & $0.6 \%$ & & $-2.0 \%$ \\
\hline 11 & $0.9 \%$ & & $-1.4 \%$ \\
\hline 12 & $1.1 \%$ & & $-0.5 \%$ \\
\hline
\end{tabular}

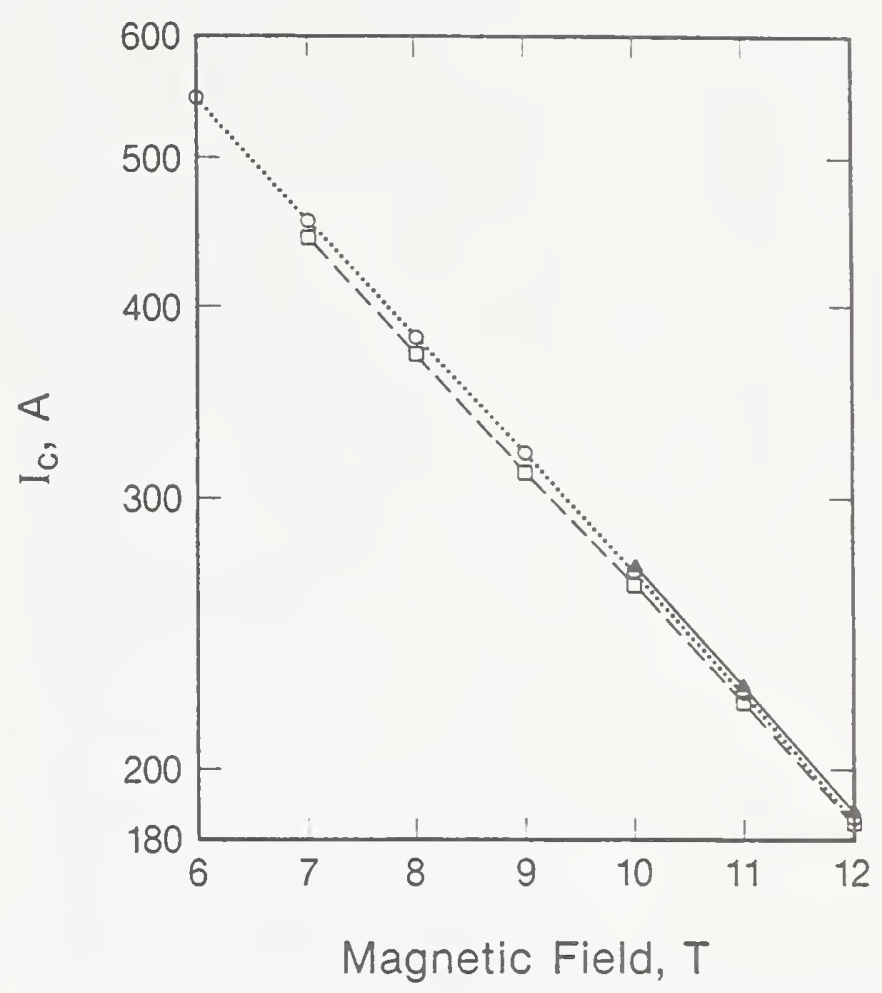

Figure 10 A semilogarithmic plot of $I_{c}$ at $10 \mu \mathrm{V} \mathrm{m}-1$ versus magnetic field for specimens of sample Z. $E_{\mathrm{c}}=10 \mu \mathrm{V} \mathrm{m}^{-1} . \Delta-\Delta$, Central $A ; \bigcirc \cdots O$, central $B ; \square---\square$, self

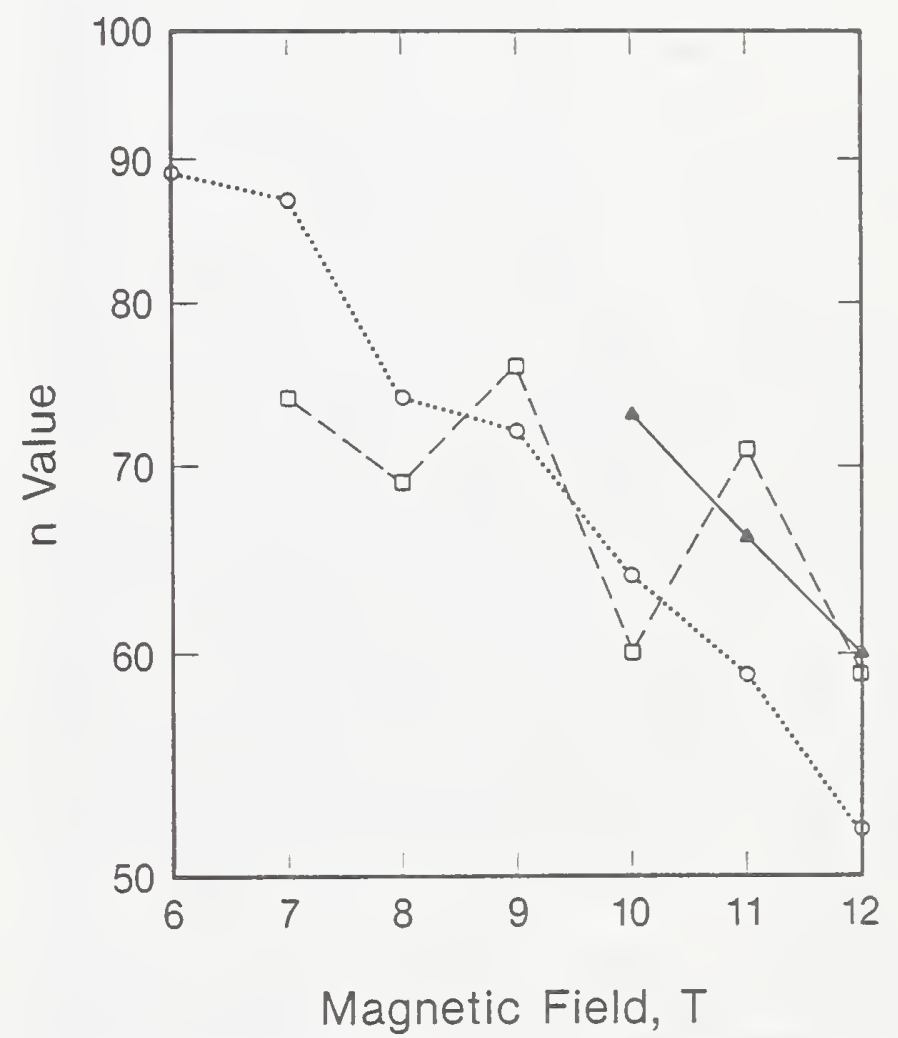

Figure 11 A semilogarithmic plot of $n$ value versus magnetic field for specimens of sample $Z$. $\Delta-\Delta$. Central A; $\bigcirc \cdots O$, central $B$; $\square---\square$, self

ment precision for this sample, homogeneity between taps was similar to the other samples.

\section{Thermal contraction measurements and strain calculations}

Thermal contraction measurements on NEMA-type (National Electrical Manufacturers' Association) G10 glass-epoxy composites have been published for the plate 


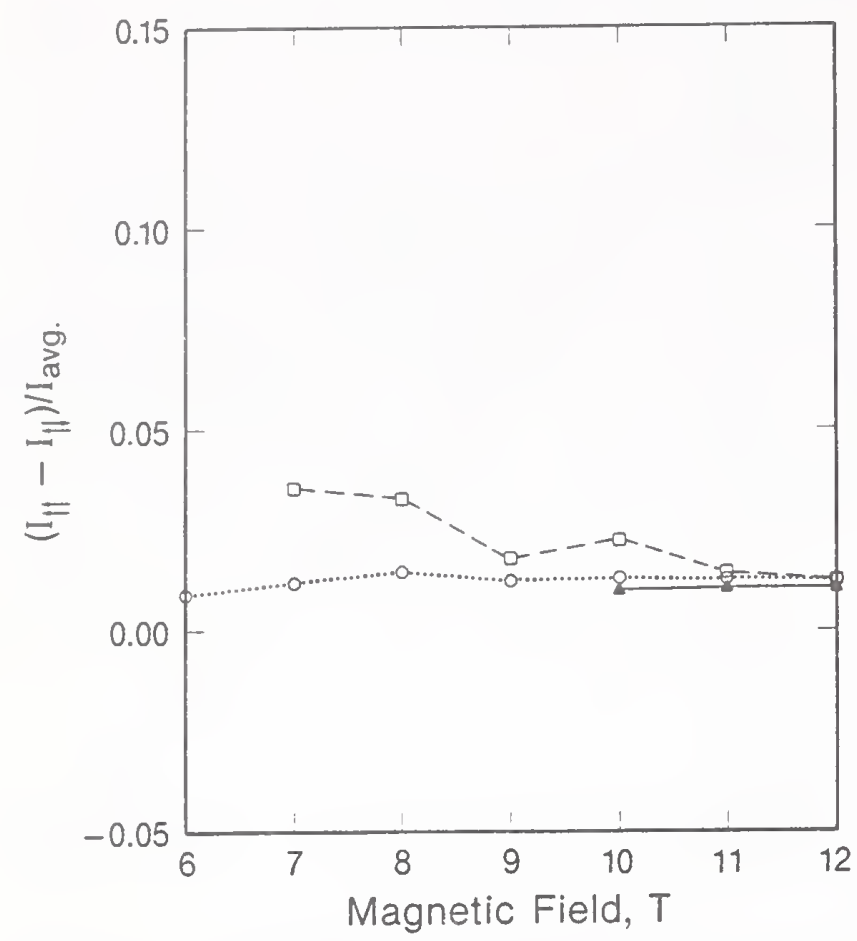

Figure 12 Percentage difference between $/ c$ measured with reverse and forward current directions versus magnetic field for specimens of sample Z. $E_{\mathrm{c}}=10 \mu \mathrm{V} \mathrm{m} \mathrm{m}^{-1}$. $\Delta-\Delta$. Central $\mathrm{A}$; $\bigcirc \cdots O$. central B: $\square--\square$, self

geometry ${ }^{5-7}$ but not for the tube geometry. The contraction of a G10 plate is highly anisotropic. In composite nomenclature, the two directions in the plane of the fibreglass fabric are warp and fill. The direction perpendicular to the plane of the fabric is referred to as the normal direction. The thermal contraction from $293 \mathrm{~K}$ to $4 \mathrm{~K}$ is about $0.24 \%$ for the warp direction and $0.71 \%$ for the normal direction. The contraction in the fill direction is expected to be a little more than that of the warp direction. The contraction in the warp direction is dominated by the fibreglass fabric and the normal direction is dominated by the epoxy. The G10 tubes used for measurement mandrels were rolled spirals of fibreglass fabric embedded in an epoxy matrix, rather than tubes machined from plate material. The radial direction for a rolled tube is normal to the fabric. The radial contraction of a tube is different than the contraction of a plate, however, because the circumferential fibreglass is put in hoop compression by the epoxy and the resulting contraction is a competition between the two structural components. The dependence of the radial contraction on wall thickness is expected to be caused by this competition.

A subset of the round robin participants used measurement mandrels from a single batch of G10 tubes to reduce the number of measurement variables among these laboratories. A thick-walled $\mathrm{G} 10$ tube was originally selected as a common sample mandrel material to allow adaptation of one size tube to the various test fixtures. However, a thick-walled tube is difficult to manufacture without delaminations that can cause irregular contraction and voids when they are machined. Also, the outer diameter can change when the inner diameter is bored. Another observation was that the radial contraction can be slightly asymmetric; this will result in an approximately elliptical rather than circular cross section at low temperatures. In order to make an approximate correction for this, the thermal contraction measurements are the average of two approximately orthogonal measurements of the tube's diameter.

As briefly mentioned above, thermal contraction measurements were made on several G10 tubes in order to estimate the resulting conductor strain. In the interest of expediency, these measurements were limited both in accuracy and precision; nonetheless, the measurements give an indication of the variation in thermal contraction between the tubes. These tests consisted of measuring the outside diameter of the G10 tube at room temperature, submerging the tube in liquid nitrogen, allowing it to reach thermal equilibrium and then quickly removing it and remeasuring the diameter. These measurements were made with a precision micrometer but they were limited by the practical difficulties of the measurement. Two tube geometries were measured; both had $31.2 \mathrm{~mm}$ nominal outside diameters, but one tube had a $12.7 \mathrm{~mm}$ inside diameter while the other had a $26.2 \mathrm{~mm}$ inside diameter. These two geometries had $9.3 \mathrm{~mm}$ and $2.5 \mathrm{~mm}$ walls, respectively, and are representative of the thick- and thin-walled mandrels. These measurements showed a substantial difference between the radial thermal contraction of G10 tubes having different wall thicknesses. Specifically, the thin-walled tube's radial thermal contraction was $\sim 0.23 \%$ while the thick-walled tube's contraction was $\sim 0.41 \%$. A bias in this study exists as a result of the thermally transient measurement conditions (with a systematic difference in thermal mass) and the fact that it was conducted at liquid nitrogen, rather than liquid helium, temperature. Both of these factors result in an underestimation of the actual thermal contraction between room and liquid helium temperatures. Based on the temperature dependence of the thermal contraction of G10 plate 5.6 , the thermal contraction to $4 \mathrm{~K}$ was estimated as $\sim 0.28 \%$ for the thin-walled tube and $\sim 0.48 \%$ for the thick-walled tube. The thermal contraction of the $\mathrm{Nb}_{3} \mathrm{Sn}$ conductor was only $\sim 0.21 \%$; consequently, the G10 contraction may have introduced additional amounts of conductor pre-strain. The amount of compressive pre-strain might depend on several factors including the mandrel's wall thickness and the strength of the mandrel-to-conductor bond.

For samples $\mathrm{X}$ and $\mathrm{Y}$, considerable $I_{\mathrm{c}}$ tensile-strainsensitivity data were available; however, no explicit data were available for compressive strain sensitivity. Consequently, the strain scaling law ${ }^{3}$ in conjunction with the available tensile strain data was used to estimate the expected $I_{c}$ degradation for compressive strain. For sample $X$ the results of these calculations predict a $\sim 33 \%$ reduction in the $I_{\mathrm{c}}$ at $12 \mathrm{~T}$ for the thick-walled specimens as compared to the thin-walled ones. As mentioned above, the observed reduction was $\sim 39 \%$. Sample $Y$ had a lower strain sensitivity with a predicted $I_{c}$ degradation of $\sim 24 \%$ at $12 \mathrm{~T}$. The actual reduction in $I_{\mathrm{c}}$ for the thick mandrel as compared to the thin was $\sim 18 \%$. As stated above, these measurements and calculations are only approximate but the trend seems to be obvious. In all cases, the thick-walled samples had reduced $I_{c} s$ and this reduction was in approximate agreement with that predicted by the strain scaling law.

\section{Discussion}

Sample damage is a major concern because it can go undetected and have a significant effect on the accuracy 
of the $I_{\mathrm{c}}$ measurement. Two potential sources of damage are shipping, when the packaging of reacted samples is inadequate, and sample transfer from the reaction mandrel to the measurement mandrel, particularly when diffusion bonding between the specimen and the stainless steel reaction mandrel has occurred.

Diffusion bonding has been observed even in cases where the surface of the stainless steel was oxidized prior to sample reaction. Consequently, a more complete and reliable technique for oxidizing the reaction mandrel needs to be developed. If specimen damage during transfer is to be avoided, dexterity and patience are required. Unthreading the specimen from a grooved mandrel seems to be better than attempting to flex the coil to a larger diameter in order to slip it over the grooves. Long coils tend to tighten as they are unthreaded unless the torque is evenly distributed among the coil turns during the process.

The other potential source of sample damage, shipping, is more easily avoided. Careful packaging of the samples can eliminate concerns over shipping damage. The reaction mandrel alone cannot protect a sample from shipping damage. The packaging needs to protect the sample from collisions with external objects as well as from inertial collisions with other specimens within the container or with the container itself.

Within this study it was not possible to separate the effect of reaction variables or sample inhomogeneity from that of shipping damage; however, considering the poor packaging that was used for some of the samples, it is suspected that sample damage may have played a role in these measurements. The damage or loss of samples during shipping and the delay of sample shipments by customs are characteristic problems of international round robin measurements and, hopefully, with experience these problems will be reduced.

The study concerning the effect of boring and thickversus thin-walled G-10 tubes was not originally intended but, rather, the results of initial measurements led naturaliy to it. Thus, the number of specimens and the schedule limited the completeness and organization of these results. A more selective data presentation was not used because it would imply that there were fewer difficulties and that less was learned in these studies than was actually the case.

A round robin is a unique measurement setting where the interlaboratory comparison of results is essential to the advancement of measurement accuracy. Although only the NBS results have been presented herein, it was the preliminary comparison between these results and results from the other laboratories that lead to the expansion of the NBS study and, ultimately, to the source of the measurement discrepancies.

\section{Conclusions}

Two major conclusions are that the bonding method and the mandrel material and geometry have a significant effect on the critical current measurement of $\mathrm{Nb}_{3} \mathrm{Sn}$. Thick wall G10 tubes contract about $0.2 \%$ more than thin wall G10 from room to liquid helium temperature. Thus, the amount of differential thermal contraction between the mandrel and the sample depends on the wall thickness of the G10. The method of bonding the sample to the mandrel also determines how much of the differential thermal contraction is transmitted to the sample. A positive bond will change the strain state of the sample more than a weak bond; however, a weak bond may allow the sample to be strained by the Lorentz force. The difference between critical current measurements on thick and thin wall G10 was about $20 \%$ at $12 \mathrm{~T}$ for a $\mathrm{Nb}_{3} \mathrm{Sn}$ conductor with an upper critical field of $24 \mathrm{~T}$ and about $35 \%$ at $12 \mathrm{~T}$ for one with an upper critical field of $19 \mathrm{~T}$.

The effect of self versus central sample reaction was not indicated as a major source of error in the resulting critical current measurements. The most direct comparison of this effect can be made on sample $\mathrm{X}$, where the effect was less than $3 \%$ at $12 \mathrm{~T}$. Furthermore, sample $\mathrm{X}$ should have been more sensitive to reaction conditions because of its relatively short reaction time, which may not have fully reacted the $\mathrm{Nb}$ filaments.

Some preliminary recommendations based on these results can be made. G10 tubes with wall thickness less than about $20 \%$ of the tube radius are well matched in thermal contraction to $\mathrm{Nb}_{3} \mathrm{Sn}$ wires and use of this type of tube for measurement mandrels should result in a minimum of conductor strain. The boring of thick-walled G10 tubes for use as measurement mandrels may, due to structural voids, result in irregular thermal contraction. A well oxidized stainless steel reaction mandrel should be used to reduce diffusion bonding of the sample to the reaction mandrel and the possibility of damage when the sample is transferred to the measurement mandrel. A thin, continuous coat of epoxy will provide a good bond between the sample and the mandrel; however, in cases where end cooling of the sample is inadequate, this method may result in a lower measured $I_{c}$. Also, from a practical standpoint other bonding materials, such as varnish or grease, that are easily removed from the measurement mandrel may be preferable to epoxy for routine measurements. These bonding materials may be adequate in cases where the measurements are conducted with the Lorentz force into the mandrel.

The results presented here are preliminary in the sense that they have yet to be correlated with measurements from all 24 participating laboratories. This correlation is a necessary element in a study of this type and may clarify the results presented here. The effect of thick- versus thin-walled measurement mandrels discovered in this study indicates that in order for various laboratories to arrive at the same $I_{\mathrm{c}}$ results, a more detailed unification of the measurement techniques would be required. However, this would not ensure the correctness of the results, only a consistency of results. A unified definition of the desired measurement, aimed at achieving the maximum usefulness and practicality of the resulting data, is required in order to make a correct measurement. Hopefully, a future joint publication will address some of these issues and result in a simple measurement method that has only the restrictions necessary to achieve this end.

\section{Acknowledgements}

The authors extend their thanks to J.W. Ekin for discussions on these results, to R.M. Folsom and T.C. Stauffer for sample preparation, data reduction and plotting, to D.L. Rule for help with data plotting, to W.E. Look for assistance with the magnetic field calibration and to the other VAMAS participants. 
This work was supported by the Department of Energy, Office of Fusion Energy and Division of High Energy Physics.

An effort was made to avoid the identification of commercial products by the manufacturer's name or label, but in some cases these products might be indirectly identified by their particular properties. In no instance does this identification imply endorsement by the National Bureau of Standards, nor does it imply that the particular products are necessarily the best available for that purpose.

\section{References}

1 Goodrich, L.F. and Fickett, F.R. Critical current measurements: a compendium of experimental results Cryogenics (1982) 22225
2 Standard Test Method for D-C Critical Current of Composite Superconductors Annual Book of ASTM Standards ASTM B714-82, Part 02.03, American Society for Testing and Materials, Philadelphia, PA, USA (1983) 595-598

3 Ekin, J.W. Strain scaling law for flux pinning in practical superconductors. Part 1: Basic relationship and application to $\mathrm{Nb}_{3} \mathrm{Sn}$ conductors Cryogenics (1980) 20611

4 Bray, S.L., Goodrich, L.F. and Dube, W.P. Battery powered current supply for superconductor measurements Rev Sci Inst (1989) 60261

5 Fujii, G., Ekin, J.W., Radebaugh, R. and Clark, A.F. Effect of thermal contraction of sample holder material on critical current Adv Cryog Eng Mater (1980) 26589

6 Fujii, G., Ranney, M.A. and Clark, A.F. Thermal expansion of $\mathrm{Nb}_{3} \mathrm{Sn}$ and $\mathrm{V}_{3} \mathrm{Ga}$ multifilamentary superconducting cables, fiberglass-epoxy and cotton-phenolic composite materials Jap J Appl Phys (1981) 20 L267

7 Clark, A.F., Fujii, G. and Ranney, M.A. The thermal expansion of several materials for superconducting magnets IEEE Trans Magn (1981) MAG-17 2316 


\section{APPENDIX F}

THERMAL CONTRACTION OF FIBERGLASS-EPOXY SAMPLE MANDRELS

AND ITS EFFECT ON CRITICAL-CURRENT MEASUREMENTS

L. F. Goodrich, S. L. Bray, and T. C. Stauffer

$$
\begin{gathered}
\text { published in: } \\
\text { Proceedings of the } 6^{\text {th }} \mathrm{Japan-US} \mathrm{Workshop} \\
\text { on }
\end{gathered}
$$

High-Field Superconducting Materials and

Standards Procedures for High-Field

Superconducting Materials Testing.

Boulder, Colorado

February 22-24, 1989

Editors: K. Tachikawa, K. Yamafuji and H. Wada and

J.W. Ekin and M. Suenaga

pp. 91-93, Japan (1989) 
Thermal Contraction of Fiberglass-Epoxy Sample Mandrels and Its Effect on Critical-Current Measurements*

L. F. Goodrich, S. L. Bray, and T. C. Stauffer National Institute of Standards and Technology Boulder, Colorado 80303

A systematic study was made of the effect of sample mandrel thermal contraction on the superconducting critical-current $\left(I_{C}\right)$ measurement. This study was conducted in conjunction with the VAMAS (Versailles Agreement on Advanced Materials and Standards) interlaboratory comparison (round robin) measurements. The $\mathrm{Nb}_{3} \mathrm{Sn}$ wire measured in this study had a diameter of $0.68 \mathrm{~mm}$. The sample coils were mounted using a filled-epoxy adhesive on mandrels that were made from fiberglass-epoxy (G-10) tubes of several different wall thicknesses.

Figure 1 is a semilogarithmic plot of $I_{c}$ versus magnetic field for three different sample mandrels, designated thick, bored, and thin. The variable among the three sample mandrels is the ratio of their radius to their wall thickness. The relative dimensions of the three mandrels are given in Table I. In all cases, the mandrels were constructed from G-10 tubes. The precision of the $I_{c}$ measurement was $\pm 0.2 \%$. The uncertainty of the $I_{C}$ measurements may be as large as $\pm 5 \%$ because of the strong systematic effects of sample mandrels.

The bored designation refers to a specimen that was first mounted and measured on the thick walled mandrel. The specimen was then brought to room temperature and the mandrel's inner diameter was bored to a larger size,

Table I. Mandrel Dimensions

\begin{tabular}{lccc}
\hline & $\frac{\text { thick }}{9.3}$ & $\frac{\text { bored }}{2.5}$ & $\frac{\text { thin }}{2.0}$ \\
Wall, mm & 15.6 & 15.6 & 15.6 \\
Rad. mm & 15.6 & 168 & 138 \\
Wall/Rad & $60 \%$ & 168 \\
\hline
\end{tabular}

* Contribution of NIST, not subject to copyright. resulting in a reduced wall thickness. The specimen's $I_{c}$ was then remeasured. On the other hand, the thin designation refers to a specimen that was simply mounted on a mandrel that had been already machined to a reduced wall thickness. The reason for the two different approaches was to demonstrate that neither sample variability nor changes in sample strain introduced during boring were principally responsible for variations in the measured $I_{C}$.

A comparison between the thin and bored specimens shows only a small difference, 3 to 48 , in $I_{c}$ with very little field dependence. This indicates that the effect of boring was about equivalent to that of simply mounting a

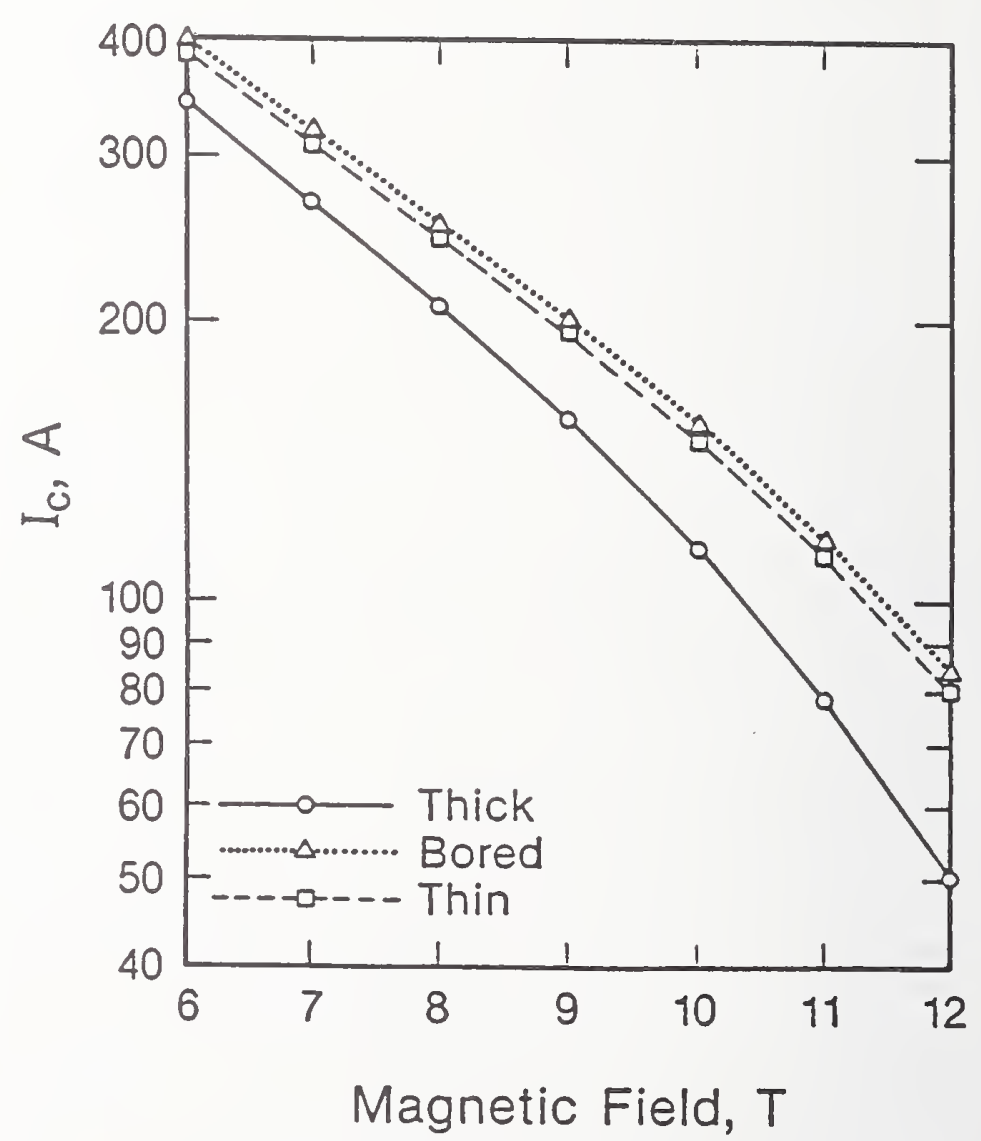

Figure 1. A semigarithmic plot of $I_{C}$ for an electric field criterion of 10 $\mu \mathrm{V} / \mathrm{m}$ versus magnetic field. 
specimen on a thin-walled tube. In comparison to the thin and bored specimens, the thick specimen shows a significant reduction in $I_{c}$; the difference between the curves increases with increasing magnetic field ( 40 \% at $12 \mathrm{~T}$ ), which is indicative of a strain effect.

The thick- and the thin-walled sample holders are made from the same materials and constructed using the same

techniques. The difference in their radial thermal contraction is a result of their highly anisotropic structure. In the case of plates that are made of this material, the thermal contraction normal (perpendicular) to the plate is considerably greater than in the plane of the plate. A competition between these two contractions occurs in the tube geometry where the direction of greater contraction is radial and the direction of lesser contraction is circumferential. Consequently, the wall thickness of the tube affects its overall radial thermal contraction.

Thermal contraction measurements were made on several G-10 tubes in order to estimate the resulting conductor strain. These measurements showed a substantial difference among the radial thermal contraction of G-10 tubes that have different wall thicknesses. The results of the thermal contraction measurements are shown in Table II. The thermal contraction was determined by measuring the outside diameter of the G-10 tube at room temperature, submerging the tube in liquid nitrogen, allowing it to reach thermal equilibrium, and then quickly removing it and remeasuring the diameter. These measurements were made with a precision micrometer, but their accuracy was limited by the practical difficulties of the measurement. The estimated uncertainty in the value of $\Delta d / d$ was \pm 10 \%. As indicated by the column headings in the right hand portion of the table, four different samples were measured.

For each sample, the table shows the tube's wall thickness, the ratio of the tube's wall thickness to its radius in percent, and the thermal contraction of the tube between room and liquid nitrogen temperatures $(\Delta d / d)$. Samples 1 and 2 were progressively bored to larger inside diameters while samples 3 and 4 had factory diameters (not machined) and were measured to test the equivalence between factory tubes and bored tubes with respect to thermal contraction. The data indicate that the thickerwalled tubes contract more than the thinner ones. The agreement of the data for samples 3 and 4 with those of 1 and 2 indicates that the bored tubes behave in a manner similar to that of factory tubes. Samples 1 and 2 with wall thickness/radius ratios of 59\%, $16 \%$, and $13 \%$ have respectively similar dimensions to the thick, bored, and thin measurement mandrels.

A bias in this thermal-contraction study exists as a result of the fact that it was conducted near liquid nitrogen, rather than liquid helium, temperature. This factor results in an underestimate of the actual thermal contraction between room and liquid helium temperatures. Based on the temperature dependence of the thermal contraction of G-10 plate, 1 the thermal contraction to $4 \mathrm{~K}$ was estimated as $\sim 0.28 \%$ for the thin-walled mandrel and

Table II. Thermal Contraction of G-10 Tubes Between Room and Liquid Nitrogen Temperatures

\begin{tabular}{|c|c|c|c|}
\hline \multicolumn{2}{|c|}{ Tube Dimensions } & \multicolumn{2}{|c|}{$\begin{array}{l}\text { Outer Diameter } \\
\text { Thermal Contraction }\end{array}$} \\
\hline $\begin{array}{l}\text { Ratio } \\
\text { Wall } \\
\text { to } \\
\text { Radius }\end{array}$ & $\begin{array}{l}\text { Wall } \\
\text { Thickness }\end{array}$ & $\begin{array}{l}\text { Sample } 1 \\
\Delta \mathrm{d} / \mathrm{d}\end{array}$ & $\begin{array}{l}\text { Sample } 2 \\
\Delta \mathrm{d} / \mathrm{d}\end{array}$ \\
\hline $\begin{array}{l}598 \\
498 \\
398 \\
28 z \\
16 z \\
13 z\end{array}$ & $\begin{array}{l}9.27 \mathrm{~mm} \\
7.70 \mathrm{~mm} \\
6.10 \mathrm{~mm} \\
4.32 \mathrm{~mm} \\
2.54 \mathrm{~mm} \\
2.03 \mathrm{~mm}\end{array}$ & $\begin{array}{l}0.398 \\
0.38 \% \\
0.33 z \\
0.30 z \\
0.25 \% \\
0.258\end{array}$ & $\begin{array}{l}0.418 \\
0.368 \\
0.328 \\
0.288 \\
0.238 \\
0.248\end{array}$ \\
\hline $\begin{array}{l}\text { Ratio } \\
\text { Wall } \\
\text { to } \\
\text { Radius }\end{array}$ & $\begin{array}{l}\text { Wall } \\
\text { Thickness }\end{array}$ & $\begin{array}{l}\text { Sample } 3 \\
\Delta d / d\end{array}$ & $\begin{array}{l}\text { Sample } 4 \\
\Delta d / d\end{array}$ \\
\hline $\begin{array}{l}20 \% \\
30 \%\end{array}$ & $\begin{array}{l}3.18 \mathrm{~mm} \\
4.76 \mathrm{~mm}\end{array}$ & $0.25 \%$ & 0.288 \\
\hline
\end{tabular}


- 0.488 for the thick-walled mandrel. The thermal contraction of the $\mathrm{Nb}_{3} \mathrm{Sn}$ conductor is only $-0.21 \%$. Furthermore, the $\mathrm{Nb}_{3} \mathrm{Sn}$ conductor was rigidly bonded to the mandrel with a filled-epoxy adhesive. Consequently, the G-10 contraction may have introduced compressive strain to the conductor.

Considerable data on the sensitivity of $I_{c}$ to tensile strain were available for this sample; however, no explicit data were available for compressive strain sensitivity. Consequently, the strain scaling law, ${ }^{2}$ in conjunction with the available tensile strain data, was used to estimate the expected $I_{C}$ degradation for compressive strain. The results of these calculations predict a -33 r reduction in $I_{c}$ at $12 \mathrm{~T}$ for the thick-walled specimens as compared to the thin-walled ones; the observed reduction was $-40 \%$. These measurements and calculations are only approximate, but the trend is apparent. In all cases, the thick-walled samples had reduced critical current and this reduction was in approximate agreement with that predicted by the strain scaling law.

G-10 tubes that have diameters similar to those tested and wall thicknesses about $20 \%$ or less of the tube's radius are well matched in thermal contraction to $\mathrm{Mb}_{3} \mathrm{Sn}$ wires. Use of this type of tube for measurement mandrels should result in a minimum of conductor strain. The scaling of this effect for tubes of various diameters has not been demonstrated, but scaling is probably accurate for a limited range of tube diameters.

This, work was supported by the Department of Energy, Office of Fusion Energy and Division of High Energy Physics.

An effort was made to avoid the identification of commercial products by the manufacturer's name or label, but in some cases these products might be indirectly identified by their properties. In no instance does this identification imply endorsement by the National Institute of Standards and Technology, nor does it imply that the products are necessarily the best available for that purpose.

\section{References}

1. G. Fujii, J. W. Ekin, R. Radebaugh, and A. F. Clark, "Effect of Thermal Contraction of Sample Holder Material on Critical Current," Adv. Cryog. Eng.-Materials, vol. 26, pp. 589-598, Plenum Press, New York (1980).

2. J. W. Ekin, "Strain Scaling Law for Flux Pinning in Practical Superconductors. Part 1: Basic Relationship and Application to $\mathrm{Nb}_{3} \mathrm{Sn}$ Conductors, " Cryogenics, vol. 20, pp. 611-624, Nov. 1980. 
THERMAL CONTRACTION OF FIBERGLASS-EPOXY SAMPLE HOLDERS USED FOR $\mathrm{Nb}_{3} \mathrm{Sn}$ CRITICAL-CURRENT MEASUREMENTS

L. F. Goodrich, S. L. Bray, and T. C. Stauffer

Presented at the International Cryogenic Materials Conference Los Angeles, California 24-28 July 1989

to be published in: Advances in Cryogenic Engineering Materials, Volume 36 


\title{
THERMAL CONTRACTION OF FIBERGLASS-EPOXY SAMPLE \\ HOLDERS USED FOR Nb ${ }_{3} S n$ CRITICAL-CURRENT MEASUREMENTS*
}

\author{
I. F. Goodrich, S. I. Bray, and T. C. Stauffer \\ Electromagnetic Technology Division \\ National Institute of Standards and Technology \\ Boulder, Colorado 80303
}

\section{ABSTRACT}

It is typical for $\mathrm{Nb}_{3} \mathrm{Sn}-\mathrm{Cu}$ superconductor specimens to be wound into coils on tubular specimen holders for critical-current measurements. If the thermal contraction of the holder is different than that of the specimen, axial strain may be applied to the specimen upon cooling from room to liquid-helium temperature. This strain can affect the measured critical current. The thermal contraction was measured for three different $\mathrm{Nb}_{3} \mathrm{Sn}-\mathrm{Cu}$ superconductors. Also, the thermal contraction was measured for several different specimen holders, all of which were made from fiberglass-epoxy composites. The specimen holder measurements were made using an electricalresistance strain-gage technique, and they were confirmed by direct mechanical measurements. The tubes varied in diameter, wall thickness, and fabrication technique. Some of the tubes were made directly from tube stock, and others were machined from plate stock. The results of these measurements show that the thermal contraction of tube stock is strongly dependent on the ratio of its wall thickness to its radius, while the contraction of tubes machined from plate stock is relatively independent of these dimensions. Critical-current measurements of $\mathrm{Nb}_{3} \mathrm{Sn}$-Cu specimens mounted on these various holders show that the presence of differential thermal contraction between the specimen and its holder can significantly affect the measured critical current.

\section{INTRODUCTION}

Critical-current $\left(I_{C}\right)$ measurements of $\mathrm{Nb}_{3} \mathrm{Sn}$-Cu superconductors can be affected by the relative thermal contraction of the material on which the test specimen is mounted. This measurement variable became apparent in the recent VAMAS (Versailles Project on Advanced Materials and Standards) interlaboratory comparative measurements (round robin) of the critical current of $\mathrm{Nb}_{3} \mathrm{Sn}_{1}{ }^{1}$ Differential thermal contraction between the specimen and its holder in cooling from room to liquid-helium temperature can cause either a tensile or compressive strain of the specimen, either of which can affect the measured $I_{C} .2,3$ For coil specimens that are mounted on the surface of cylindrical holders, the strain is predominantly along the axis of the specimen. The amount of strain depends on the magnitude of the

\footnotetext{
ॠContribution of NIST, not subject to copyright.
} 
differential contraction, the relative strengths of the specimen and holder, and the mechanical coupling between the specimen and its holder.

The literature contains considerable data on the compressive pre-strain of $\mathrm{Nb}_{3} \mathrm{Sn}$ filaments caused by differential thermal contraction between the filaments and the matrix material. However, very little data on the overall thermal contraction of $\mathrm{Nb}_{3} \mathrm{Sn}$-Cu wires are presently available. The thermal contraction of a $\mathrm{Nb}_{3} \mathrm{Sn}$-Cu cable is given in Ref. 4; however, this conductor has a tungsten core that reduces its thermal contraction. Consequently, the thermal contraction of three different $\mathrm{Nb}_{3} \mathrm{Sn}$ wires was measured as a part of this study.

Fiberglass-epoxy composites are commonly used sample holder materials. These materials are anisotropic in three mutually perpendicular directions. The three directions are associated with characteristics of the fiberglass fabric, and they are designated as the warp, fill, and normal directions. The fabric orientation for plate stock is shown in Fig. 1. The normal direction is perpendicular to the fabric planes, while the warp and fill directions are parallel to the fabric planes. The density of the fabric is not the same in both directions of the weave. The warp and fill directions are determined by the fabric's thread count. The number of threads per unit length of fabric is lower in the warp direction than in the fill direction. This structural anisotropy causes a three-dimensional variation in thermal contraction. The contraction in the fill direction is slightly greater than in the warp direction, but the contraction in the normal direction is considerably larger. ${ }^{4,5}$

Figure 1 also shows the typical fabric orientation for a rolled tube. The radial thermal contraction of the tube results from a competition between the larger contraction in the normal direction and the smaller contraction in the warp direction. Consequently, it depends on the ratio of the tube's wall thickness to its outside radius (wall-to-radius ratio). For thin-walled tubes the radial thermal contraction approaches that of a plate in the warp direction, and for thick walled tubes it approaches that of a plate in the normal direction.

A holder whose thermal contraction is relatively independent of its wall-to-radius ratio can be made by machining a cylindrical tube from thick fiberglass-epoxy plate stock with the axis of the tube perpendicular to the surface of the plate (plate tube). For this orientation (Fig. 1) the radial contraction is based on the contraction in the warp and fill directions,

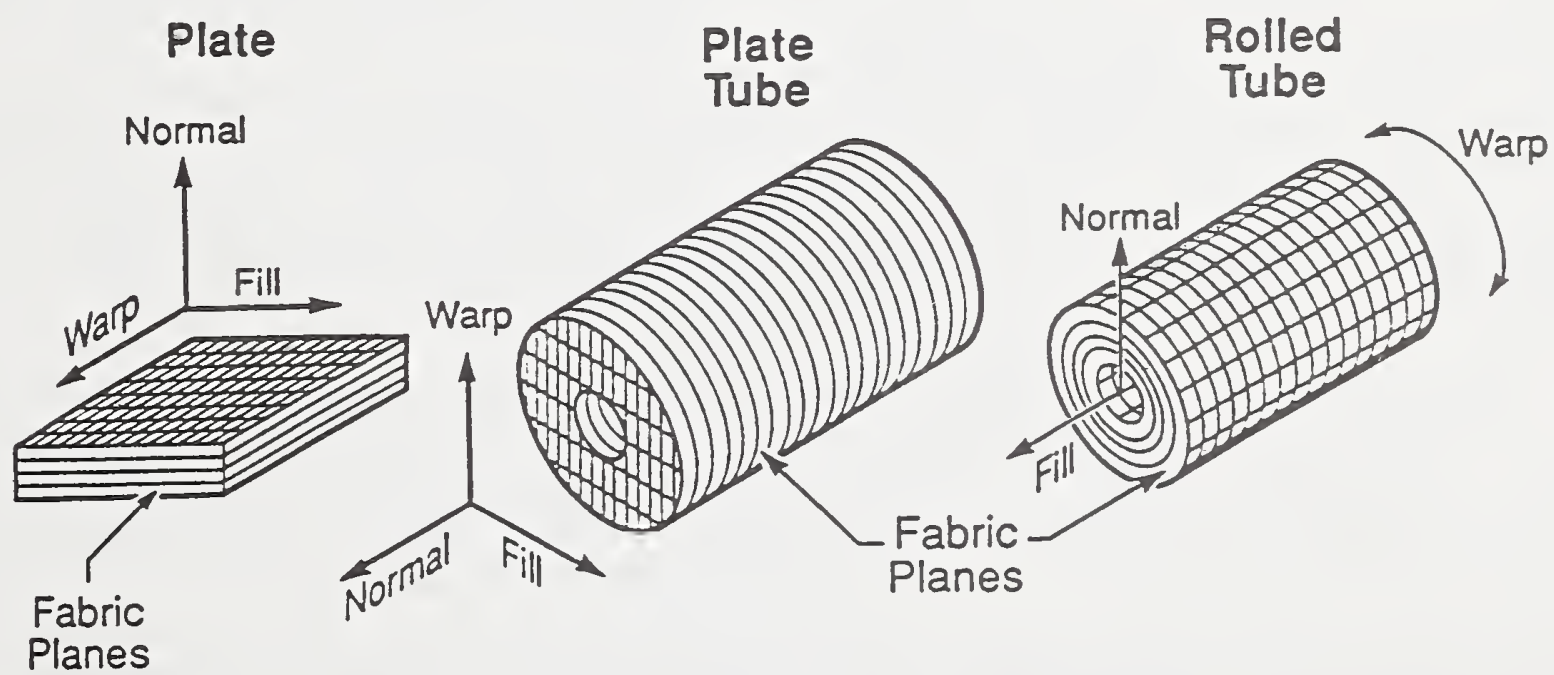

Fig. 1. Geometries of fiberglass-epoxy composites. 
which are both similar to that of a $\mathrm{Nb}_{3} \mathrm{Sn}$-Cu wire. In addition to thermal contraction measurements of both rolled tubes and plate tubes, I $c$ measurements were made using both types of specimen holder to confirm the relationship between thermal contraction and the $I_{c}$ measurement.

\section{EXPERIMENTAL DETAILS}

\section{Fiberglass-Epoxy Composites}

Two different types of fiberglass-epoxy composite were used in this study, NEMA (National Electrical Manufacturers' Association) G-10 and G-11. All of the rolled tubes were G-10 and the plate tubes were G-11. This was not a matter of choice but, rather, one of material availability. The thermal contraction of the G-11 is slightly less than that of G-10. Neither of these materials was cryogenic-radiation (CR) grade, which is designated as G-10CR or G-11CR. The manufacturing specifications for the $\mathrm{CR}$ grade materials are more stringent and their performance at cryogenic temperatures is more predictable. 5

\section{Thermal Contraction}

All of the thermal contraction measurements were conducted between room temperature and liquid-nitrogen ( $\mathrm{LN}_{2}$ ) temperature. We assume that, if a material's contraction is well matched to a $\mathrm{Nb}_{3} \mathrm{Sn}$-Cu wire from room to $\mathrm{LN}_{2}$ temperature, then the additional differential thermal contraction that occurs between $\mathrm{LN}_{2}$ and liquid-helium temperature will be insignificant.

The $\mathrm{Nb}_{3} \mathrm{Sn}-\mathrm{Cu}$ thermal contraction measurements were made using quartz reference tubes and reacted $\mathrm{Nb}_{3} \mathrm{Sn}$-Cu specimens that measure approximately $35 \mathrm{~cm}$ in length. The specimen is placed in a quartz tube and attached to one of the tube's ends. At room temperature, the quartz tubes are approximately $1 \mathrm{~mm}$ longer than the specimens. This differential length is measured with a micrometer, the tube and specimen are cooled to $\mathrm{LN}_{2}$ temperature, and the differential length is remeasured. The thermal contraction of the specimen is deduced from the measured change in the differential length and the known contraction of the quartz tube. The uncertainty of these measurements is estimated to be $\pm 5 \%$.

Two different methods were used to measure the thermal contraction of the fiberglass tubes. The first was a mechanical method, where the tube was submerged in liquid nitrogen, allowed to reach thermal equilibrium, removed from the nitrogen, and the diameter was then quickly measured with a precision micrometer. To address the thermally transient nature of these measurements, the tube's diameter was measured as a function of time while it was warming toward room temperature. These measurementa allowed an extrapolation of the data to liquid-nitrogen temperature. In the case of the plate tubes, two orthogonal measurements were made at each time, one in the warp direction and one in the fill direction. The uncertainty of the mechanical measurements of thermal contraction was estimated to be $\pm 10 \%$ of the measured value (for example, $0.20 \% \pm 0.02 \%$ ).

The second type of thermal contraction measurement used electricalresistance strain gages. ${ }^{6}$ For this measurement, two well matched $350 \Omega$ strain gages were used in a half-bridge configuration. One of the gages was bonded to the test specimen and the other was bonded to a reference material, a quartz tube, whose thermal contraction was known. All of the strain-gage measurements reported here were taken with the strain gages mounted on the circumference of the test specimen since this represents the contraction which is relevant for the $\mathrm{Mb}_{3} \mathrm{Sn}-\mathrm{Cu}$ coil. Both the test specimen 
and reference material were cooled to liquid nitrogen temperature and the resulting output from the strain bridge was measured.

Two factors will cause a change in the resistance of the strain gages when cooled from room to $\mathrm{LN}_{2}$ temperature. First, the resistivity of the strain gages' grid alloy will change with temperature. Since this change in resistance will be nearly equal for matched strain gages, there will be little effect on the output of the strain bridge. The second source of change in resistance is caused by thermally induced strain. This strain results from differential thermal contraction between the strain gage and the test specimen. If the thermal contraction of the test specimen material is different than that of the reference material, the strain bridge will be unbalanced and its output will be indicative of the difference in thermal contraction between these two materials. Since the thermal contraction of the reference material is known, the thermal contraction of the test material can be deduced. The uncertainty of the strain-gage measurements of thermal contraction was estimated to be $\pm 5 \%$ of the measured value (for example, $0.208 \pm 0.018$ ).

\section{Critical Current}

For the $I_{c}$ measurements, a $\mathrm{Nb}_{3} \mathrm{Sn}-\mathrm{Cu}$ wire with a diameter of 0.68 wn was used. This conductor was made by an internal-tin diffusion process, and it has 37 sub-bundles of $150 \mathrm{Nb}$ filaments. A single Ta diffusion barrier separates the filament region from the outer $C u$ layer. The specimen was wound onto a stainless steel tube and then vacuum heat treated at $700^{\circ} \mathrm{C}$ for $48 \mathrm{~h}$. The stainless steel tube has a helical groove machined into its surface to retain the specimen and define its geometry. Following heat treatment, the specimen was removed from the stainless steel reaction holder and transferred to the fiberglass measurement holder. The outside diameter of the fiberglass holder is $3.12 \mathrm{~cm}$ and its surface is not grooved. A thin continuous layer of filled epoxy adhesive was painted over the surface of the specimen and holder. The typical specimen length was approximately $80 \mathrm{~cm}$. Three pairs of adjacent voltage taps were placed along the center of the specimen. Each pair had a separation of about $10 \mathrm{~cm}$, and there was a 1 to $2 \mathrm{~cm}$ gap between adjacent pairs. An electric field criterion of $10 \mathrm{\mu V} / \mathrm{m}$ was used for determining the critical current. The uncertainty of the $I_{c}$ measurements may be as large as \pm 5 o because of the strong systematic effects of the specimen holders.

\section{RESULTS}

\section{Thermal Contraction Measurements}

The results of the thermal contraction measurements are shown in Fig. 2 where the thermal contraction of the tubes' diameters $(\Delta d / d)$ is plotted as a function of the tubes' wall-to-radius ratios. The thermal contraction of three different superconductors (the VAMAS $I_{C}$ round robin conductors ${ }^{1}$ ) was also measured. The dashed horizontal line labeled " $\mathrm{Nb}_{3} \mathrm{Sn}$-Cu" indicates the measured thermal contraction $\left(0.28 \%\right.$ ) of the conductor whose $I_{c}$ was measured in this study. The measured thermal contraction of the other two conductors is $0.28 \%$ and $0.26 \%$. The two types of data symbols that are labeled "Mechanical" and "Strain Gage" show the thermal contraction of the G-10 rolled tube specimens as measured by the two different techniques. With the exception of the "Mechanical" data, all of the data shown in this figure were obtained using the strain-gage technique.

The data show a strong dependence of the thermal contraction on the wall-to-radius ratio. The approximate thermal contraction of G-10CR plate stock in cooling from room to liquid-nitrogen temperature is $0.224 \%, 0.264 \%$, 
and $0.687 \%$ in the warp, fill, and normal directions respectively, and it is $0.202 \%, 0.227 \%$, and $0.585 \%$ for $\mathrm{G}-11 \mathrm{CR} .4,5$ As expected from the structural geometry of the rolled tubes, their thermal contraction fall between that of the warp and normal directions of plate stock. The measured thermal contraction of the G-11 plate tubes is shown for three wall-to-radius ratios and in two structural directions, warp and fill. In both directions, the thermal contraction is relatively independent of the wall-to-radius ratio and it is comparable to that of plate stock. The error bars associated with the high wall-to-radius ratio data points represent the range of values that were measured on three different specimens with three repeat determinations for each specimen.

Additional measurements were made using strain gages that were mounted on the circumference of the plate tube halfway between the warp and fill axes. The thermal contraction at these positions was within the experimental uncertainty of the value measured in the warp direction. This indicates that the effective circumferential thermal contraction of the plate tube is closer to the value measured for the warp direction. Mechanical thermal contraction measurements were also made on the plate tubes and were within the experimental uncertainty of the strain-gage measurements. Additional thermal contraction measurements were made on tubes with different outer diameters to test the scaling of thermal contraction with the wall-to-radius ratio. All of the data of Fig. 2 were taken on tubes having outer diameters of about $3.18 \mathrm{~cm}$ and various inner diameters. However, two rolled tubes with outer diameters of 11.4 and

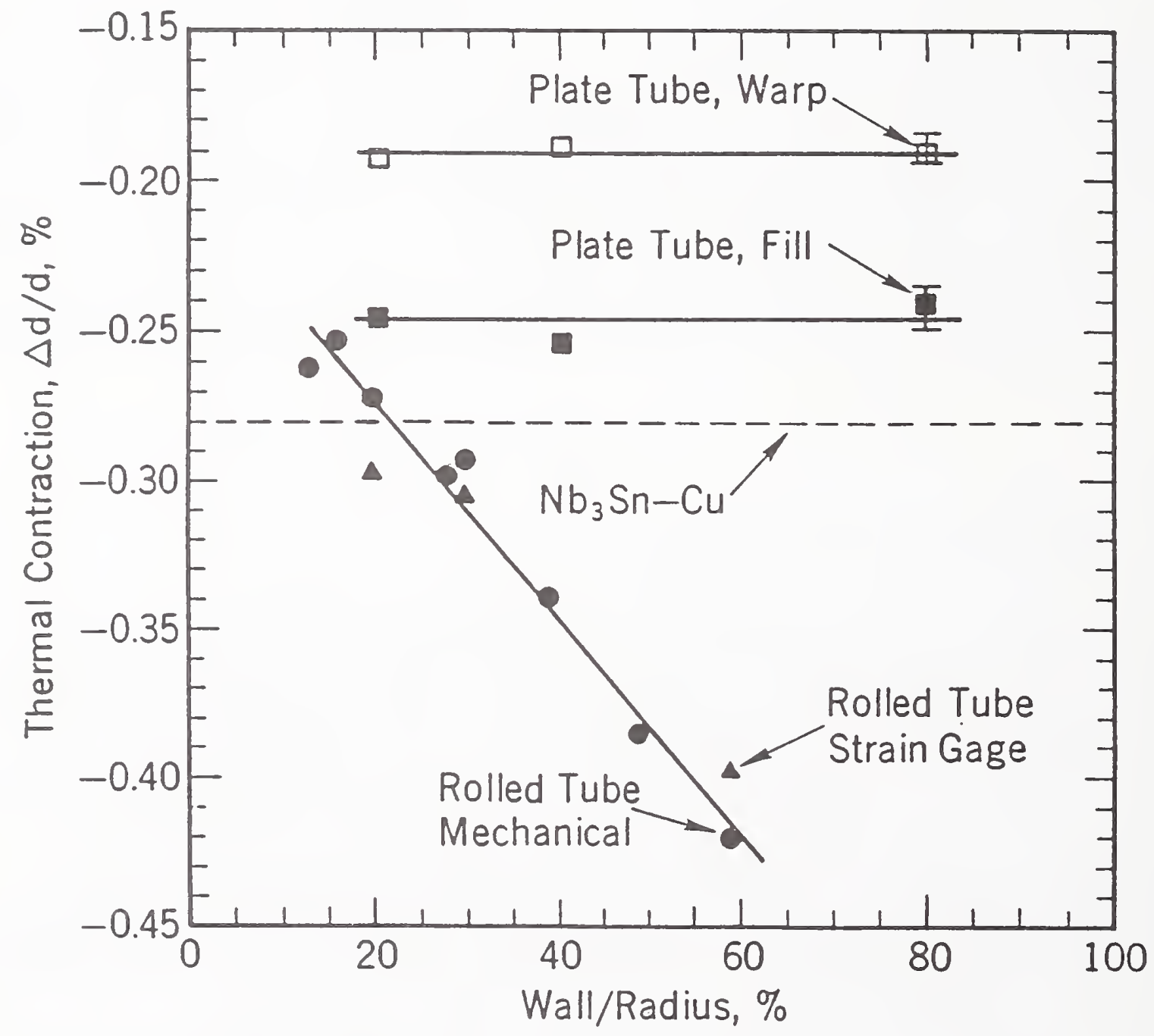

Fig. 2. Plot of room to liquid-nitrogen temperature thermal contraction of G-10 rolled tubes and G-11 plate tubes as a function of the tubes' wall-to-radius ratio. 
$12.4 \mathrm{~cm}$, and wall-to-radius ratios of 14 and $8 \%$, respectively, were also measured. The thermal contraction of these two tubes was within the experimental uncertainty of the rolled tube data of Fig. 2. Finally, an $11.4 \mathrm{~cm}$ outer diameter ( 948 wall-to-radius ratio) plate tube was measured and was within the experimental uncertainty of the plate tube data of Fig. 2 .

\section{Critical-Current Measurements}

The results of the $I_{c}$ measurements are shown in Fig. 3, where the $I_{c}$ is plotted as a function of applied magnetic field for several different conductor specimens and specimen holders. There are three variables, in addition to the applied magnetic field, for these $I_{c}$ measurements: the location where the specimen was reacted, "central" or "self"; the specimen holder's wall-to-radius ratio, "thick" or "thin"; and the type of tube used for the specimen holder, "rolled" or "plate". The self reacted samples were reacted at NIST, whereas the central reacted samples were reacted at another site and shipped to NIST for $I_{c}$ measurements. The thick and thin designations do not indicate specific wall-to-radius ratios; instead, they indicate two general categories of tube geometry. All of the thin tubes have wall-to-radius ratios that are no greater than $13 \%$

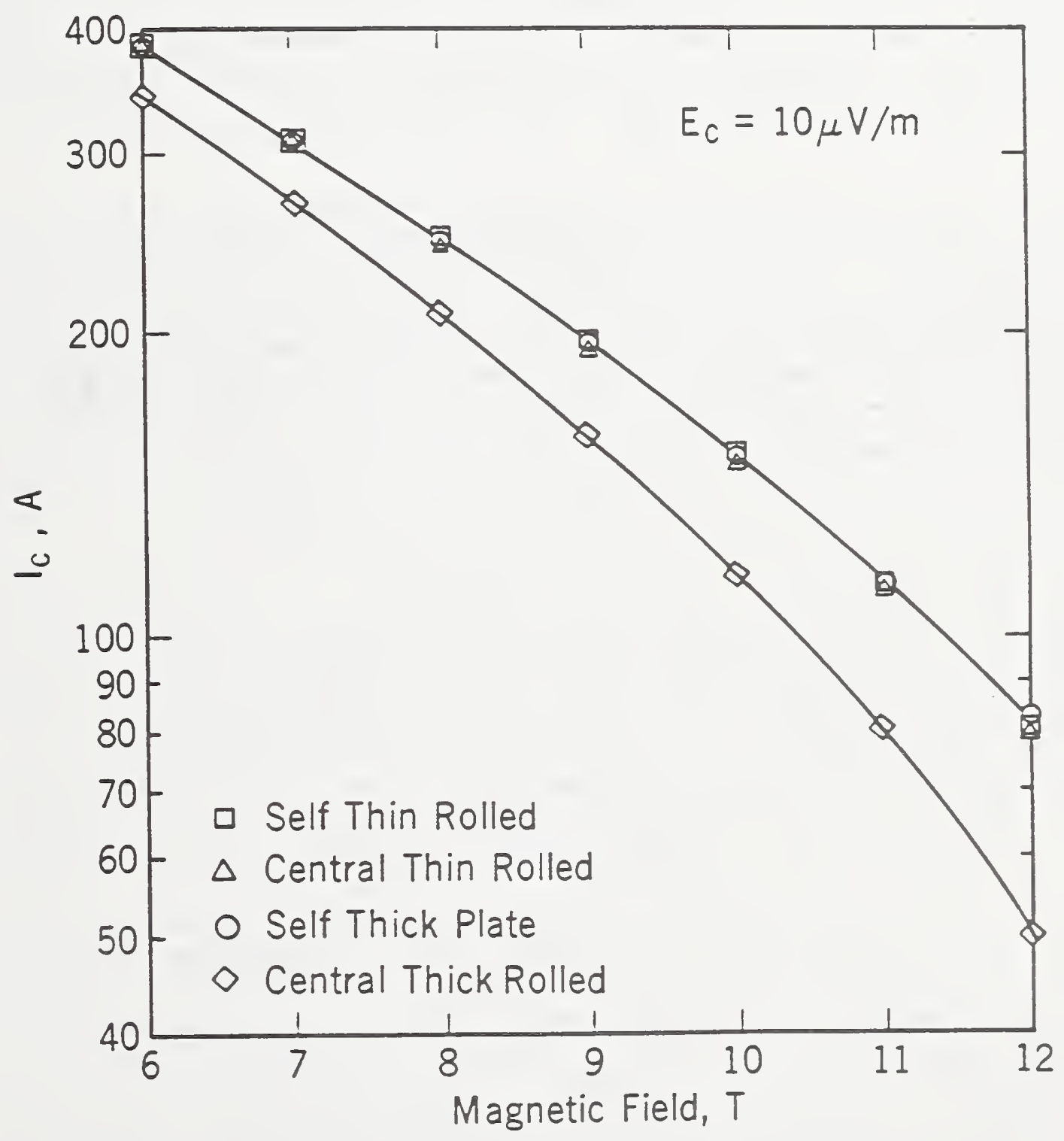

Fig. 3. A semilogarithmic plot of $I_{c}$ at an electric field criterion of $10 \mu \mathrm{V} / \mathrm{cm}$ as a function of magnetic field. 
(self 108 and central 138) and the thick tubes have wall-to-radius ratios that are at least 608 (plate $80 \%$ and rolled 60\%). The measured $I_{C}$ 's are nearly the same, within experimental uncertainty, for all of the specimens except the one that was mounted on the thick, rolled tube. The measured $I_{c}$ is significantly lower for this specimen at all magnetic fields. The difference is about $14 \%$ at $6 \mathrm{~T}$ and 408 at $12 \mathrm{~T}$. The fact that the $I_{c}$ degradation increases with increasing magnetic field is consistent with a strain effect. The magnitude of the change in $I_{c}$ and the measured thermal contraction are in good agreement with the strain effect, 3 At $12 \mathrm{~T}$, a $33 \%$ reduction was calculated from strain-effect measurements ${ }^{1}$ that were made at $14 \mathrm{~T}$, and the measured $I_{C}$ reduction was 408 .

\section{DISCUSSION}

A practical reality of round robin measurements is that the dimensions of the specimen holders vary between different laboratories. For consistent measurements, the specimen holders should be designed so that the $I_{c}$ measurement is insensitive to this variable. The thermal contraction of a tubular specimen holder that is made from an anisotropic material can vary with its geometry. This presents the potential for variations in the strain state of the specimen and, thus, variations in the measured $I_{c}$. An apparent solution to this problem is to use an isotropic material for the specimen holders and to use a bonding technique that rigidly couples the specimen to its holder. This will ensure that the strain transmitted to the specimen, due to thermal contraction, is independent of the holder's geometry and, thus, equivalent from laboratory to laboratory. This approach addresses the issue of measurement consistency, but it does not address accuracy.

The $I_{c}$ measurement should, arguably, be made with a minimum of externally applied strain on the superconductor. This requires a strong bond between the specimen and holder to avoid specimen strain under the influence of the Lorentz force, and it requires that the thermal contraction of the sample holder be well matched to that of the superconductor. Also, the holder should, ideally, be made from an electrically insulating material to prevent current sharing with the test specimen. Unfortunately, an isotropic and insulating material with a thermal contraction similar to that of $\mathrm{Nb}_{3} \mathrm{Sn}-\mathrm{Cu}$ is not readily available. Fiberglass-epoxy plate tubes are a practical alternative to the ideal isotropic specimen holder. The thermal contraction of a plate tube is slightly anisotropic; however, it is relatively independent of the tube's dimensions. Furthermore, the thermal contraction in the radial direction (the pertinent direction for a coil-type specimen) is similar to that of $\mathrm{Nb}_{3} \mathrm{Sn}-\mathrm{Cu}$. Based on the thermal contraction of G-1OCR and G-11CR plate, 4,5 the thermal contraction of G-10 plate tubes may be slightly closer to that of a $\mathrm{Nb}_{3} \mathrm{Sn}$ - Cu wire than the $\mathrm{G}-11$ plate tubes that were measured here. It is possible that the difference in the circumferential thermal contraction between the warp and fill directions will result in a spatial variation of the strain state of the $\mathrm{Nb}_{3} \mathrm{Sn}$-Cu coil sample, but the $I_{c}$ data indicate that this effect is not significant.

The plate tubes present some practical disadvantages. First of all, machining a specimen holder from plate stock is considerably more difficult than from tube stock. Also, the length of a plate tube is limited by the thickness of the available plate stock. This, in turn, limits the length of the superconductor specimen for a given coil diameter and pitch. Furthermore, short specimen holders are often incompatible with existing $I_{c}$ test fixtures. Satisfactory specimen holders of greater length could perhaps be constructed by bonding a series of short plate tubes together. This technique might require an alignment of the warp and fill fibers betyeen individual tube sections because of the anisotropic radial thermal contraction of plate tubes. 
For $I_{c}$ measurements, fiberglass-epoxy composites are suitable specimen holder materials. However, the design of the specimen holder should take into account the anisotropic nature of the material and the resulting variability in thermal contraction. These characteristics of the material can result in large variations in the measured $I_{c}$ for specimens mounted on holders of different designs. A cylinder or tube can be machined from a thick fiberglass-epoxy plate with the axis of the cylinder perpendicular to the surface of the plate. This type of tube (plate tube) has a thermal contraction that is relatively independent of its dimensions and that is similar to that of a $\mathrm{Nb}_{3} \mathrm{Sn}-\mathrm{Cu}$ specimen. Alternatively, the specimen holder can be made from tube stock and machined to a wall-to-radius ratio that results in a thermal contraction tha: closely matches that of the $\mathrm{Nb}_{3} \mathrm{Sn}$-Cu specimen.

\section{ACKNOWIEDGEMENTS}

The authors acknowledge the contribution of R. L. Spomer, S. Bird, and $T$. Harris who made some strain-gage measurements on the G-10 rolled tubes as part of a Colorado School of Mines class project. The authors extend their thanks to $M$. Thoner (Vacuumschelze) for a discussion of the plate tube geometry, to R. M. Folsom for sample preparation and assistance with the measurements, to J. D. McColskey for instructions on mounting strain gages, and to $R$. Gerrans for drafting.

This work was supported by the Department of Energy, Office of Fusion Energy and Division of High Energy Physics.

An effort was made to avoid the identification of commercial products by the manufacturer's name or label, but in some cases these products might be indirectly identified by their particular properties. In no instance does this identification imply endorsement by the National Institute of Standards and Technology, nor does it imply that the particular products are necessarily the best available for that purpose.

\section{REFERENCES}

1. K. Tachikawa, K. Itoh, H. Wada, D. Gould, H. Jones, C. R. Walters, L. F. Goodrich, J. W. Ekin, and S. L. Bray, VAMAS intercomparison of critical current measurement in $\mathrm{Nb}_{3} \mathrm{Sn}$ wires IEEE Trans. Magn. 25-2:2368 (1989).

2. G. Fujii, J. W. Ekin, R. Radebaugh, and A. F. Clark, Effect of thermal contraction of sample holder material on critical current Adv. Cryog. Eng. 26:589 (1980).

3. J. W. Ekin, Strain scaling law for flux pinning in practical superconductors. Part 1: basic relationship and application to $\mathrm{Nb}_{3} \mathrm{Sn}$ conductors Cryogenics 20:611 (1980).

4. A. F. Clark, G. Fujji, and M. A. Ranney, The thermal expansion of several materials for superconducting magnets IEEE Trans. Mag. MAG-17:2316 (1981).

5. M. B. Kasen, G. R. MacDonald, D. H. Beekman, Jr., and R. E. Schramm, Mechanical, electrical, and thermal characterization of G-10CR and G-11CR glass-cloth/epoxy laminates between room temperature and $4 \mathrm{~K}$ Adv. Cryog. Eng. 26:235 (1980).

6. Measurements Group, Inc., Tech Note-513, Measurement of thermal expansion coefficient using strain gages (1986). 

APPENDIX $\mathrm{H}$

VAMAS INTERCOMPARISON OF CRITICAL CURRENT MEASUREMENT IN $\mathrm{Nb}_{3} \mathrm{Sn}$ WIRES

K. Tachikawa, K. Itoh, H. Wada, D. Gould, H. Jones

C. R. Walters, L. F. Goodrich, J. W. Ekin, and S. L. Bray

IEEE Trans. on Magnetics 25(2) 2368-2374 (March 1989) 
VAMAS INIERCOMPARISON OF CRIIICAL CURRENI MEASUREMENT IN $\mathrm{Nb}_{3} \mathrm{Sn}$ WIRES

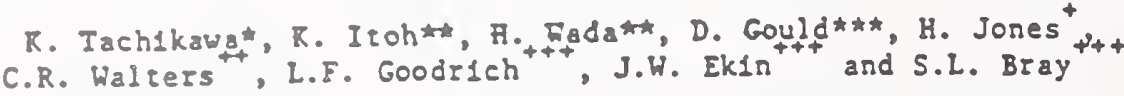

^ Ioka1 Univers1ty, H1ratsuka, Kanagawa 259-12, Japan

* Nat1onal research Inst1ture for Metals. Isukuba, Ibarak1 305, Japan

***Comission of the European Comunities, Brussels, B-1049, Belg1um +Clarendon Laboratory, Oxford, OXl 3PU, Unired K1ngdom

++ Rutherford Appleton Laboratory, DIdcot, OXII OQX, UnIted KIngdom ++ Nat1onal Bureau of Standards, Boulder, Co 80303, USA

\section{Abseract}

The VAYAS technical working party in the area of superconducting and eryogenle structural materlals has recently carrled out the flrst world-wlde intercomparison of critlcal current, I, measurement on multifllamentary $\mathrm{Nb}_{3} \mathrm{Sn}$ wres. Three sample wires vere supplied from each of EC (European Comunitles), Japan and USA. The rotal number of participant labs vere 24 (EC 11, Japan 8 and USA 5). There were few restrictions for the I measurenent at partelpant labs. The standard deviations of the I values reported from these labs varled among test samples, and were $6-21 \%$ of averaged If's at 12 Tesla.

\section{Introduction}

The superconductivity technology may have tremendous smpact on 1mportant areas of sclence and technology and should be developed under the concept of the long term project whereby international cooperation would play an essent1al role. Ihis is the underlying 1dea in the VAWAS wich stands for the Versa1lles Projects among sumlt partlelpant countrles on Advanced Materlals and Standards. The VAMAS Technical horking Parry (IhP) on superconducting and cryogenic siructural waterlals consists of representatives of particlpant countries. The InP has carried out an intercomparison on the critical current, I, measurement in $\mathrm{Nb}_{3} \mathrm{Sn}$ multifllamentary wres: I 'is the most Important superconducting parameter from the practical polnt of viet.

The purpose of the present intercomparison (round rob1n) test on $I_{\text {f }}$ is to ldent1fy parameters affect1ng the I by accumulating and evaluating the weasurement resulks. The eventual goal of the research is to provide recomendations for the performance of short sample crit1cal-current measurements of $\mathrm{Nb}_{3} \mathrm{Sn}$ superconductors.

\section{Partlclpants, Samples and Test Procedure}

11 EC, 8 Japanese and 5 US laboratorles lisied 10 Iable I have particlpated in the round robin test on the I. The distribution of test sarples and accumulation of resulting I dara in EC, Japan and USA were perforwed by the respective central labs;

BCIN(Belg1ux), NRIY, and NBS.

Hultifllamentary Nib $5 n$ wires with relatively saall eurrent carrylng capacity ( 500 A at 8 Iesla) were chosen as the test samples which could easily be tested at any partlclpant laboratory. Three sample wres were supplied, one from each of EC, Japan and USA; these samples are labeled disorderly just as sample A, B and $C$ in this paper.

Yanuscrift recelved August $22,1988$.
Sample A fabricated by a bronze method has a wre dlameter of 0.8 and 114 sub-bundles each containing $90 \mathrm{dib}-\mathrm{Ta}$ fllaments in a bronze matrix. The $\mathrm{Cu}$ siabilizer is located at the center of the wife and separated from the fllament region by a Ia barrier. The volume fraction of $\mathrm{Cu}$ stabll1zer in the wire is much saller compared to those in other 2 samples.

Sample $B$ has a vire diameter of 1.0 mm and 7 subbundles each separared by a Nib barrler from the Cu stab1l1zer ours1de. Each sub-bundle contairs 721 kb f1laments in a Cu-Sn-II alloy matrix. This sample was also fabrlcated by a bronze process.

Sample C prepared by an Internal-Sn diffusion method has a wire dlameter of 0.68 in and 37 sub-bundles each contalning $150 \mathrm{Nb}$ fllaments. The f1lament reglon $1 \mathrm{~s}$ separated from the outer layer of $\mathrm{Cu}$ by a single $\mathrm{Ia}$ barrier.

Spectflcarlons and the cross sectional views of these saxples are given in F1g l. Upper crit1cal flelds, Heq, for sample $A$ and sample $B$ are enhanced by

Table I. Partielpant laboratorles in I round robin test.
Europe (11)

Atoninst1tut der Oesterre1chischen Un1v. (Austria)

Inst. Exper1mental Phys1k, Oester. Un1v. (Austria)

S.C.K./C.E.N (Belglum)

S.N.C.I., C.N.R.S. (Erance)

Kernforschungszentrum Karlsruhe (ERG)

S1emens AG (FRG)

Vacuumschrelze GobH (FRG)

E.N.E.A, Centro d1 Erascat1 (Italy)

High Fleld Magnet Lab., U. N1jmegen (Netherlands)

Clarendon Laboratory (LK)

Rutherford Appleton Laboratory (UK)

Japan (8)

Electrotechnical Laboratory

Furukawa Electrle Co., Ltd.

Eltachl Ltd.

I.M.R., Tohoku UnIvers1ty

I.S.I.R., Osaka UnIversity

Japan Aromic Energy Research Inst1tute

Kobe Steel, Ltd.

National Research Institute for Metals

USA (5)

Brookhaven Nat1onal Laboratory

F.B.N.M.L., Massachusetrs Institute of Technology

Lawrence Livermore Nat1onal Laboratcry

National Bureau of Standards

University of hisconsin 
addilloos of Ia and I1, respecilvely.

Specimen Hres from these camples, each several weters long, were distributed to participant labs through relevant central labs. Such wres were cut into parts as specimens at each participant lab and one specimen, wound on a heat treatwent holder of drum shape, was collected from partlclpant labs and heat treated at the relevant central labs (central

reaction). The reacted specimens were then returned to the particlpant labs for weasurewents. In some cases, the part1c1pant labs also performed the1r oun heat treatments on additional speciaens of the samples (self reaction). The self reaction of samples was not possible at all labs.

Spec1f1cations of heat trearment holders were different frow $1 \mathrm{ab}$ to $\mathrm{lab}$. At most of the labs stalnless steel tubes with a splial groove on the outer surface were used to have a definite coll pltch. The surfaces of such tubes were usually coated with cerarics or oxid1zed prlor to speciuen mounting, in order to avold reaction of the specimen with the holder. At some lats the same holders were used for both hear treatwent and measurezent, thereby reducing the possibility to dacage the specimen by handing.

Central reaction wes carried out in the following wanner. For samples $A$ and $B, a l l$ speciwens were heat treated in one vacuum furnace at once. The temperature of the furnaces was well controlled within $\pm 5^{\circ} \mathrm{C}$ with cloe and in space. For sample $C$, specinens were individually heat treated in a dynanlc vacuua. Both encs of a specinen of internal-Sn processed sasple $C$ ve:e extended to a position where the temperature las kept below the melting polnt of $\mathrm{Sn}$ in order to avo1d the outflow of wolten $S \pi$ frow the wire. The heat treasent condlitons for samples $A, B$ and $C$ were $700^{\circ} \mathrm{C}$ for $96 \mathrm{hr}$, $670^{\circ} \mathrm{C}$ for $200 \mathrm{hr}$ and $700^{\circ} \mathrm{C}$ for $48 \mathrm{hr}$, respectively.

More than half of self reactions were done in vacuum. At other labs specluens were encapsulated and heat ireated $1 \pi$ an argon or a hydrogen atmosphere. The

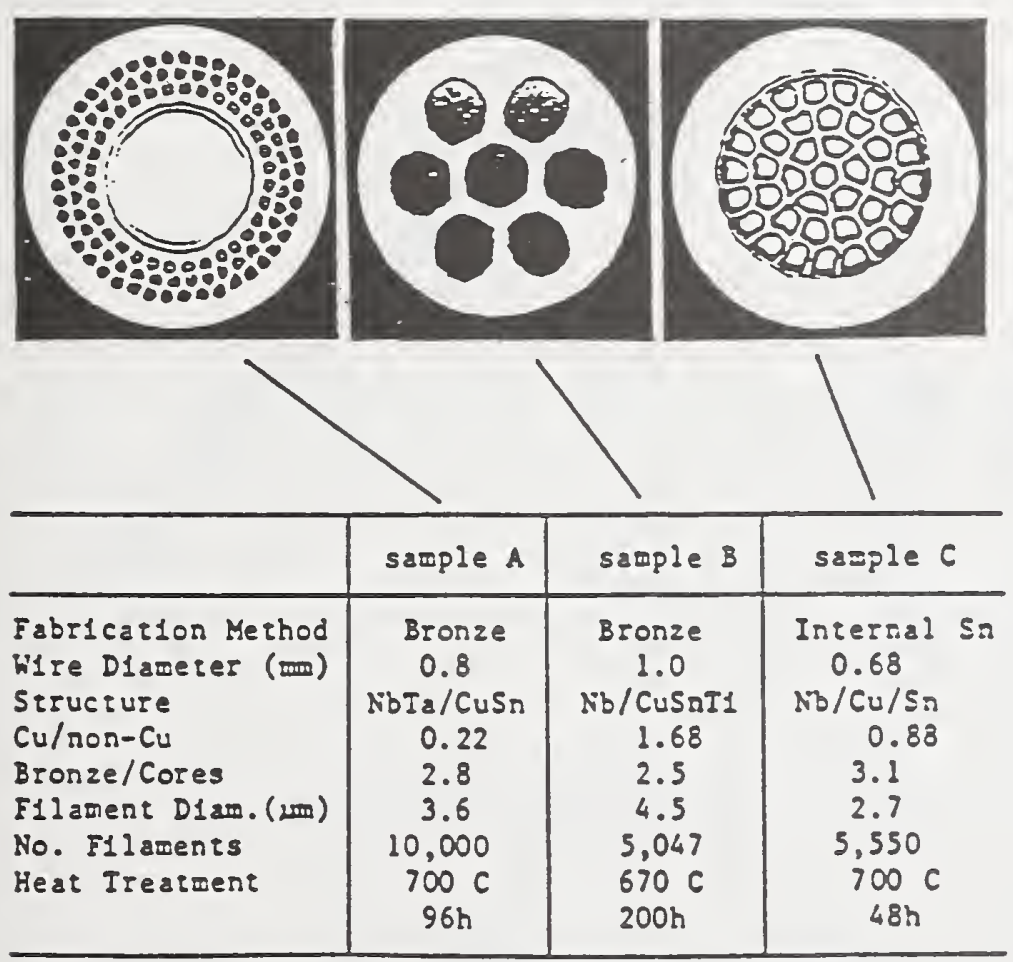

F18.1 Spectelcallo.ns and cross sectional views of round robin test samples. heat treatment temperature was also well regulared in the self reaction.

\section{Appazaruses and Measurement Conditions}

Informat1ons about weasurement apparatuses and experimental cond1tions at each laboratory were described in formatied sheets prepared and distribuied prior to the measurements.

At most of part1c1pant labs superconducting solenoid wagnets were used to generate flelds of $8-16.5$ Iesla. At sowe labs copper solenold magnets or hybrid type wagnets were used which can generate flelds over 20 Iesla. In any of these magnets mas ripple flelds which may cause heat generation in the specinen, were less than $0.1 \%$ of the fleld generated. Fleld cetertination was carried out by means of N.R, Hall probe or rotaring coll agnetometer, with estimated accuracies of 0.17 to $1 \%$.

At most of the labs transistor type poier sources were used to supply electric currents to the specinen. ro.s ripple nolses were less than $0.1 \%$ of the full pover. The accuracy of current determination was wihin $0.5 \%$ at most of the labs. In order to amplefy the voltage signals generated in the specimen, nanovoltweters were used at wost of the labs. At some labs the signals were directly led to high sensitive XY recorders. The rypical level of nolses observed varled frow $0.01 \mathrm{\mu V}$ to $2 \mathrm{LV}$, depending on the appazatuses used.

The total length of a specimen, the distance between voltage taps and the distance between a curtent tap and 1:s nearest voltage tap were much varled anong labs. The shortest specimen used in a lab had a toral length of about 215 an and 1.5 turns of winding on the weasurement holder. The longest speciaen used was 2000 long and had 30 turns on the holder.

The varlety of materlals, e.g., flbezglass relnforced plastlc (IRP), sialnless steel, alualna, hastelloy and brass were used as the measurement holder. The

Table II. Howogenelty study on sample A at SCK/CE.. Averages and standard deviations of I 's for flelds of $7-10$ Tesla.

\begin{tabular}{lr|rrrr}
\hline Fleld (Tesla) & \multicolumn{1}{c|}{7} & \multicolumn{1}{c}{8} & \multicolumn{1}{c}{9} & \multicolumn{1}{c}{10} \\
\hline Average (A) & 424.5 & 354.8 & 296.6 & 246.4 \\
Std. Dev. (A) & 6.0 & 5.8 & 5.0 & 5.3 \\
Std.Dev/Ave(Z) & 1.5 & 1.7 & 1.7 & 2.4 \\
\hline
\end{tabular}

Iable III. Howogenelty stucy on saep?e B at Clarendon lab. Averages and standard deviations of $I_{c}$ 's for flelds of $8-14$ Tesla.

\begin{tabular}{lr|rrrr}
\hline Fleld (Iesla) & \multicolumn{1}{|c}{8} & \multicolumn{1}{c}{10} & \multicolumn{1}{c}{12} & \multicolumn{1}{c}{14} \\
\hline Average (A) & 303.8 & 214.1 & 150.9 & 102.3 \\
Sid. Dev. (A) & 2.5 & 2.8 & 1.6 & 1.3 \\
Sid.Dev/Ave(Z) & 0.8 & 1.3 & 1.0 & 1.3 \\
\hline
\end{tabular}


holders had ring or bar shaped current terminals of copje:. Lost of holders had a splral groove on them. The spes:-en was mounted in a groove on the bolde: with bo:h ends solde:ed to current rerwinals, and fixed using a bond such as grease, varnish, epoxy, and solder. In some cases no bond was used.

The Lorentz force caused by the interaction of the transport current with the applled fleld has an essenilal effect on the $I_{f}$. This force acts as a tensile of compessive stress to specinen when the spiral specinen generates a central fleld parallel or antiparallel to the direction of the applied fleld. At most labs, the cirection of both flelds were antiparallel. The effect of the fleld drection on the $I_{c}$ was examined at several labs.

The I was defined at a current where a cerrain voltage gradient or a certaln resistivity appeared along the superconducting spectnen. Values of I at 5 , 10 , and $100 \mathrm{\mu V} / \mathrm{m}$ and at integer numbers of magnetic flelds were requested ro be reported $1 \mathrm{n}$ the $p$ present round robin rest; I values at $10^{-14}$ and $10^{-13} \Omega$ were opilonal.

The relationship between the voltage $V$, and the transport current, I Is emplically expressed as $v \propto I^{n}$

where the exponent $n$ is nearly constant in the small voltage reglon. A larger n corresponds to a sharper transition in the specimen. I values were also reques:ed to be reported. In the case there $n$ values vete not reported by the partielpant, they were esilmated by using the following relation, $n=1 / \log I_{c}(100 \nu \mathrm{V} / \pi) / I_{c}(10 \mu \mathrm{V} / \mathrm{m})$.

\section{Homogene1ty Study}

As the purpose of this test was the 1ntercomparison of results obtalned at different labs, it was absolutely Important that all the test specimens supplled should have 1dentical superconducting propertles. It Is difficult, however, to fabrlcate a sample with homogeneous propertles along the wtole length of a wre because of the complicated siructure of $\mathrm{Nb}_{3} \mathrm{Sn}$ mulitfllamentary composite. The homogenelty in superconducting properties was examined on sample $A$ at SCK/CEN and on sample $B$ at Clarendon Lab.

The homogenelty study at SCK/CEN was performed on 21 specimens of sample A. Specimens were raken from the vallous parts of a test wire, each wound on a holder and heat rreated at Rutherford Lab rogether with those of central reaction. The I measurement was carried out at $4.3 \mathrm{~K}$ and at 7-10 Iesia.

115 I data deflned at a voltage of $10 \mathrm{\mu V} / \mathrm{m}$ for 21 speclaens were analyzed at SCK/CEN. For voltages larger than 10uV/a spectmens showed a tendency to quenching. 15 spectmens quenched under a voltage of 10uv/m. The quench eurrents were usually lower compared to the rrue I's expected, and were not taken Into account in the statistical ireatment of the I. data. For each magnet1c fleld averages and standard deviations of I's were calculated according to the $10 u V / m$ voltage and listed in Iable II. Standard devlailons are $2.4 \%$ of average of Ic's at 10 Iesla and decrease to $1.5 \%$ at 7 Tesla.

The homogenelty in sample $B$ was examined at Clarendon Lab. 7 specluens were taken from the rest wire, each rounted onto atalniess steel holder, and heat treared at Rutherford Lab al together in a furnace. The I measurements vere carrled out at $4.2 \mathrm{~K}$ and at $7-1 \mathrm{~s}$ Iesla. Averaged If's at a voltage of $10 \mathrm{\mu V} / \mathrm{m}$ and standard deviatlons are sumarized in Table III.

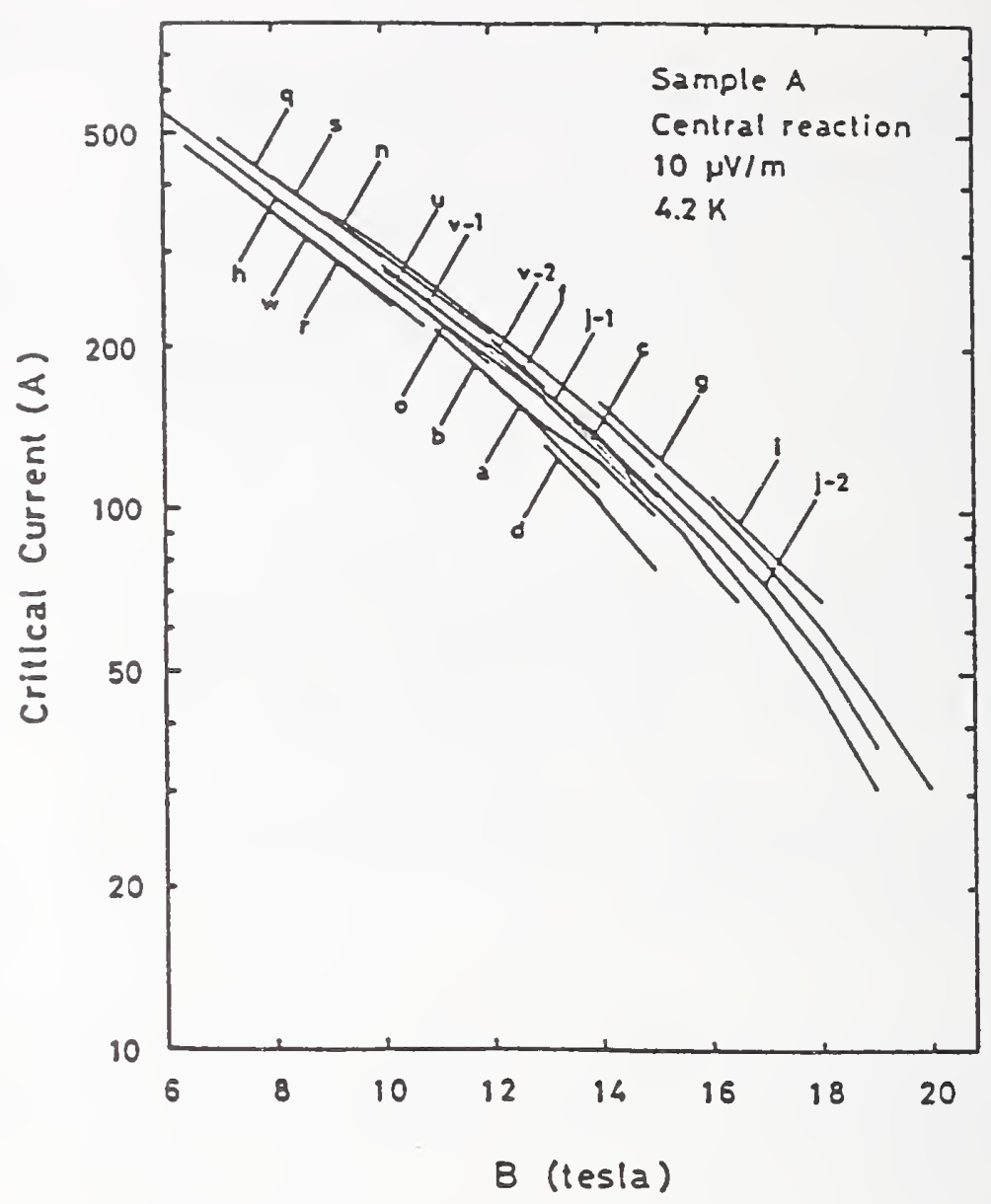

Fiz.2 I''s at $10 \mu \mathrm{V} / \mathrm{w}$ as a function of field for sáple $A$ of central reaction; results of partelpant labs.

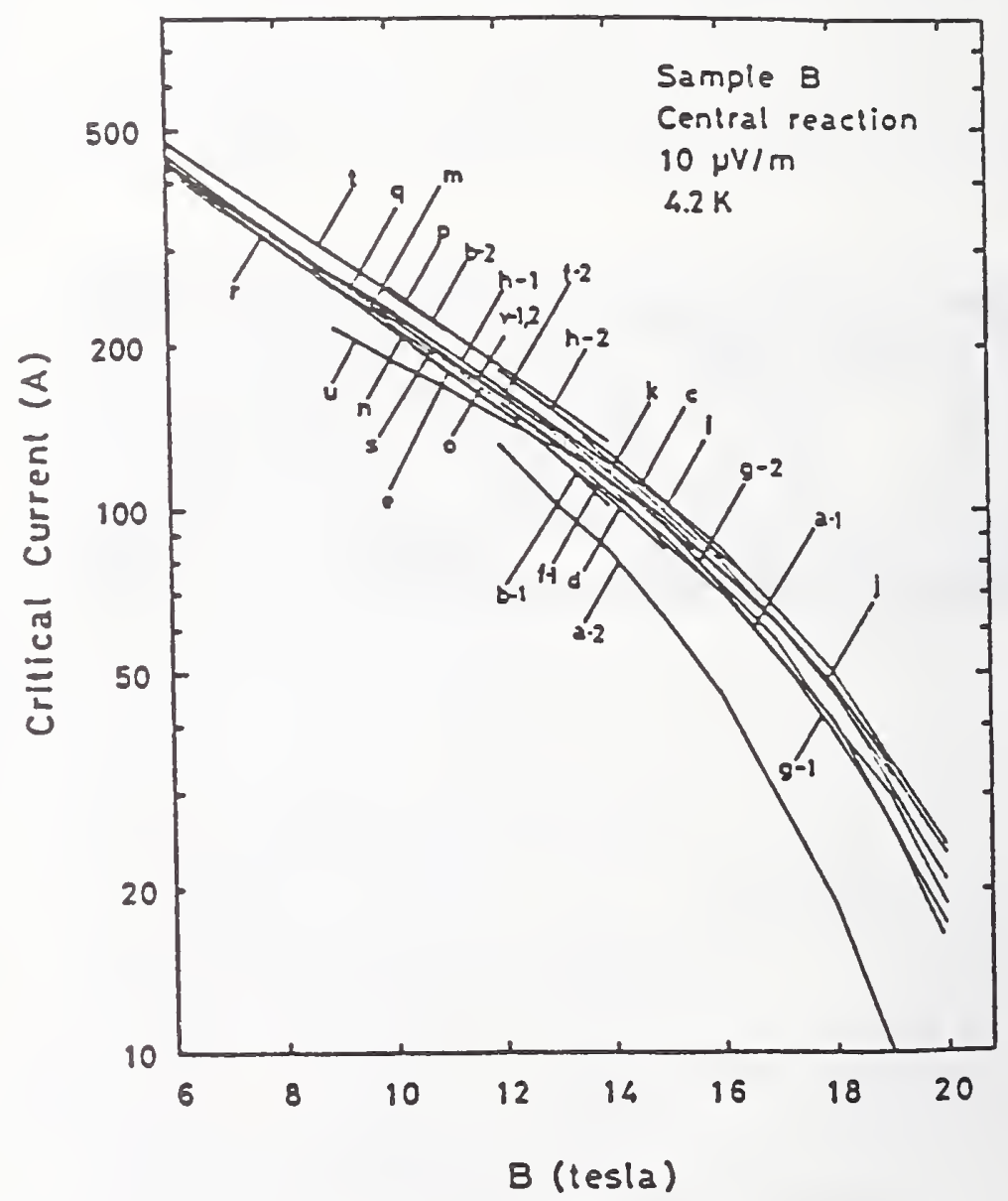

F1g.3 Ic's at $10 \mathrm{\mu V} / \mathrm{m}$ as a function of fleld for sample $B$ of central reaction; results of pariclpant labs. 


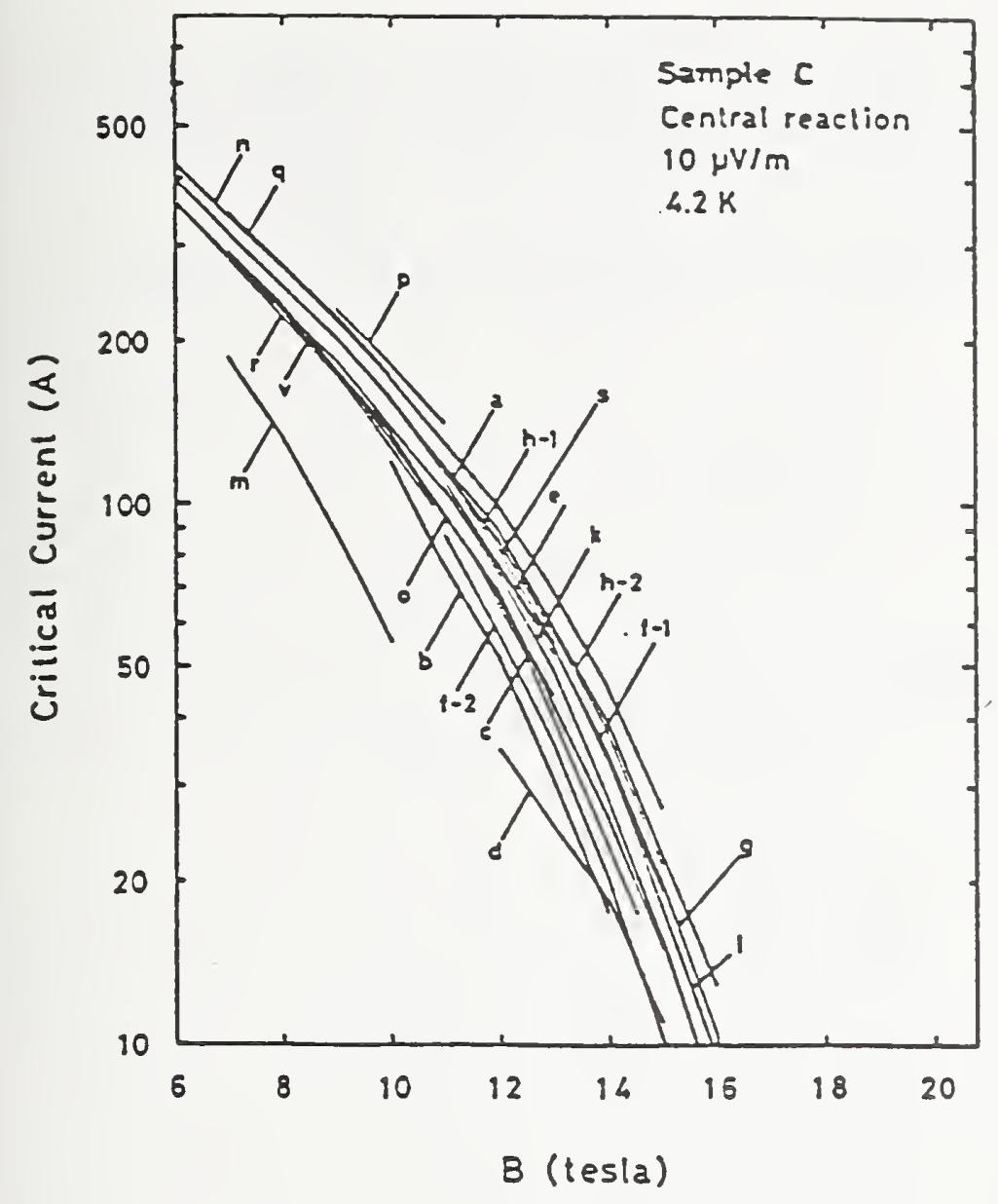

F1g.4 I 's at $10 \mathrm{rV} / \mathrm{m}$ as a function of fleld for sample $C$ of central reaction; resules of parilcipant labs.
Siandard deviations are within 1.3\% at all fields.

Based on these howogenelty studles, it may be concluded that the present test samples $A$ and $B$ are rather homogeneous in superconducting propertles over the whole length of wire and adequate for the round robin rest. The homogenelity study has not yet been carted out on sasple $C$.

\section{Results of the Round Roblin Iest}

In F1gs 2, 3 and $4 \mathrm{I}$ 's at $10 \mathrm{LV} / \mathrm{m}$ are shown as a function of applied fleld for samples $A$, $B$ and $C$, respectively. The name of labs is coded like $a, b, c$ in no special order. These resulis were obtained in the antiparallel self fleld except for those of labs and $s$.

The serllogarithaic plots show that for samples $A$ and $B$ the logs of the $I$ decreases almos: linearly biti Increasinz fleld up to about 15 Tesla, and then drops off more rapidly above this fleld. I's in sample C decrease in a similar way but with a steeper slope, and siart to drop off above abour 12 Iesla. The averaged n values generally decrease with increasing magnetic fleld and are wuch more scattered than the I 's, especially for sample A.

In Tables IV and $V$ are shown averages and standard deviations of $I$ data and a values obtafned at pazifeipant labs, Tespectively. Four extraordinarily outlying data seis vere excluded 1n the calcularions because these data sets were obralned in cases where the specimens sivek to the reaction holders and were likely camaged in rezoval or for other sialiar reasons. There was no single magnetic fleld where all labs reported data on any sample. A future publication compazing all data sers a: a single magnetic field, by means of a Kramer extrapolarton method, is planned. Coefficien:s of variation of I 's at $10 \mathrm{\mu V} / \mathrm{m}$ and 12 Iesla are abou: 7,6 and $21 \%$ of the averages for samples $A, B$ and $C$ of central reaction, respectively, and become large: a: higher magnetle fields. These values are apprestably larget than those obtalned in the homogeneity studies which are 2.4 and $1.3 \%$ fur samples $A$ and $B$, respecItvely.

Iable IV. Averages and standard deviatlons of erlitical currents at $10 \mathrm{wV} / \mathrm{m}$ for samples $A, B$ and $C$ of central reaction.

\begin{tabular}{|c|c|c|c|c|c|c|c|c|c|c|c|c|}
\hline \multirow[b]{2}{*}{$\begin{array}{l}\text { Field } \\
\text { (Iesla) }\end{array}$} & \multicolumn{4}{|c|}{ sample $A$} & \multicolumn{4}{|c|}{ sample B } & \multicolumn{4}{|c|}{ sample $C$} \\
\hline & No. & $\begin{array}{l}\text { Ave. } \\
\text { (A) }\end{array}$ & $\begin{array}{l}\sigma \\
(A)\end{array}$ & $\begin{array}{c}\sigma \text { /Ave } \\
(z)\end{array}$ & No. & $\begin{array}{l}\text { Ave. } \\
\text { (A) }\end{array}$ & $\begin{array}{l}\sigma \\
(A)\end{array}$ & $\begin{array}{r}\sigma \text { /Ave } \\
\text { (z) }\end{array}$ & No. & $\begin{array}{l}\text { Ave } \\
\text { (A) }\end{array}$ & $\begin{array}{l}\sigma \\
(A)\end{array}$ & $\begin{array}{c}\sigma \text { /Ave } \\
\text { (z) }\end{array}$ \\
\hline 6 & 1 & 544.1 & 0.0 & 0.0 & 4 & 447.4 & 18.7 & 4.2 & 3 & 393.8 & 25.7 & 6.5 \\
\hline 9 & 4 & 447.9 & 26.2 & 5.9 & 7 & 372.2 & 15.4 & 4.1 & 7 & 309.3 & 24.3 & 7.9 \\
\hline 8 & 5 & 388.7 & 21.8 & 5.6 & 8 & 315.2 & 13.0 & 4.1 & 6 & 248.6 & 20.8 & 8.4 \\
\hline 9 & 7 & 327.4 & 23.3 & 7.1 & 10 & 265.3 & 11.1 & 4.2 & .9 & 197.3 & 20.6 & ; 10.4 \\
\hline 10 & 9 & 277.1 & 18.0 & 6.5 & 15 & 229.1 & 13.1 & 5.7 & 11 & 150.0 & 18.5 & 12.3 \\
\hline 11 & 7 & 234.4 & 14.3 & 6.1 & 13 & 195.3 & 12.6 & 6.5 & 13 & 106.9 & 16.2 & 15.1 \\
\hline 12 & 11 & 195.7 & 13.6 & 6.9 & 17 & 163.6 & 10.4 & 6.3 & 16 & 72.5 & 15.2 & 20.9 \\
\hline 13 & 10 & 158.0 & 14.5 & 9.2 & 14 & 134.6 & 7.2 & 5.3 & 13 & 45.4 & 10.7 & 23.6 \\
\hline 14 & 10 & 130.5 & 15.5 & 11.9 & 16 & 114.3 & 7.8 & 6.8 & 13 & 30.0 & 8.4 & 28.0 \\
\hline 15 & 10 & 106.7 & 12.9 & 12.1 & 12 & 92.2 & 5.0 & 5.4 & 10 & 16.9 & 5.0 & 29.4 \\
\hline 16 & 6 & 91.9 & 10.8 & 11.8 & 10 & 75.8 & 4.8 & 6.4 & 7 & 8.5 & 2.6 & 30.5 \\
\hline 17 & 3 & 71.8 & 6.3 & 8.8 & 4 & 57.5 & 2.5 & 4.4 & 2 & 3.4 & 0.2 & 6.4 \\
\hline 18 & 4 & 57.4 & 9.0 & 15.7 & 7 & 43.7 & 2.6 & 8.4 & 2 & 2.1 & 0.7 & 33.7 \\
\hline 19 & 3 & 36.8 & 5.3 & 314.4 & 4 & 30.6 & 2.6 & 8.4 & 1 & 1.0 & 0.0 & 0.0 \\
\hline 20 & 2 & 26.4 & 4.4 & 16.7 & $s$ & 19.4 & 2.7 & 13.9 & & & & \\
\hline
\end{tabular}

No:number of data, $\sigma$ istandard deviation, 
In F1g. 5 I 's at $10 \mathrm{\mu V} / \mathrm{m}$ and at 12 Tesla reported froc labs are conpared. In cases no I values yere reporied at 12 I. they vere estimated by extrapolations not exceeding $2 \mathrm{~T}$. In the figure, $\Delta I_{f} / \sigma$ denotes the difference in $I$ between $l a b$ and average normalized to the standard feviation, $\sigma$. labs are arranged in incremental order of mean laboratory value of $\Delta I_{c} / \sigma$. The arrous attached to some of the data syabols Indicate that these data actually lie elther above or below the vertical lintes DI the plot. The maximum to minimum spread in $I_{\text {at }}$ each lab is almost within 2 times of $\sigma$, rather small compared to the total maximum to minimum spread (6 times of $\sigma$ ). These results elearly 1ndlcate systematic dffferences among the labs in the measured I of each sample. These differences may be due to the

Table V. Averages and standard deviarlons of n values for samples $A, B$ and $C$ of central reaction.

\begin{tabular}{|c|c|c|c|c|c|c|c|c|c|c|c|c|}
\hline \multirow[b]{2}{*}{$\begin{array}{l}\text { Fleld } \\
\text { (Iesla) }\end{array}$} & \multicolumn{4}{|c|}{ sample A } & \multicolumn{4}{|c|}{ sample B } & \multicolumn{4}{|c|}{ sample C } \\
\hline & No. & Ave. & $\sigma$ & $\begin{array}{c}\text { /Ave } \\
(z)\end{array}$ & No. & Ave. & $\sigma$ & $\begin{array}{r}\sigma / \text { Ave } \\
(\pi)\end{array}$ & No. & Ave & $\sigma$ & $\begin{array}{r}\text { /Ave } \\
(\bar{z})\end{array}$ \\
\hline 6 & & & & & 4 & 37.2 & 2.4 & 6.5 & 3 & 32.2 & 2.8 & 8.5 \\
\hline 7 & & & & & 6 & 39.3 & 5.0 & 12.8 & 7 & 32.8 & 7.6 & 23.3 \\
\hline 8 & 4 & 78.3 & 33.9 & 43.4 & 8 & 36.8 & 5.6 & 15.2 & 6 & 32.5 & 4.9 & 14.9 \\
\hline 9 & 5 & 87.8 & 38.5 & 43.8 & 10 & 34.2 & 4.5 & 13.1 & 9 & 28.6 & 3.5 & 12.1 \\
\hline 10 & 7 & 58.0 & 21.4 & 36.9 & 13 & 32.6 & 5.5 & 16.8 & 11 & 26.7 & 5.2 & 19.3 \\
\hline 11 & 6 & 50.0 & 23.3 & 46.7 & 13 & 32.3 & 8.2 & 25.3 & 13 & 26.8 & 8.8 & 32.8 \\
\hline 12 & 9 & 52.7 & 18.7 & 35.5 & 17 & 30.3 & 6.6 & 21.8 & 16 & 21.9 & 7.7 & 35.0 \\
\hline 13 & 8 & 43.9 & 16.5 & 37.7 & 15 & 28.3 & 7.5 & 26.7 & 13 & 18.7 & 7.4 & 39.7 \\
\hline 14 & 7 & 35.1 & 14.8 & 42.3 & 16 & 27.8 & $\cdot 6.2$ & 22.2 & 13 & 14.5 & 5.1 & 34.9 \\
\hline 15 & 6 & 37.3 & 13.1 & $35: 1$ & 12 & 28.1 & 5.1 & 18.0 & 10 & 12.4 & 3.5 & 28.5 \\
\hline 16 & 3 & 33.0 & 3.6 & 10.8 & 10 & 25.9 & 4.0 & 15.4 & 7 & 10.5 & 2.7 & 25.5 \\
\hline 17 & & & & & 4 & 24.2 & 5.3 & 21.9 & 2 & 8.8 & 1.6 & 17.7 \\
\hline 18 & 1 & 22 & 0.0 & 0.0 & 7 & 20.6 & 4.3 & 20.9 & 3 & 6.3 & 0.7 & 10.8 \\
\hline 19 & & & & & 4 & 19.0 & 3.5 & 18.2 & 1 & 5.2 & 0.0 & 0.0 \\
\hline 20 & & & & & 5 & 12.8 & 2.3 & 17.8 & & & & \\
\hline
\end{tabular}

No:numer of data, $\sigma$ istandard deviation $\sigma$ /Ave:coefficient of varlation

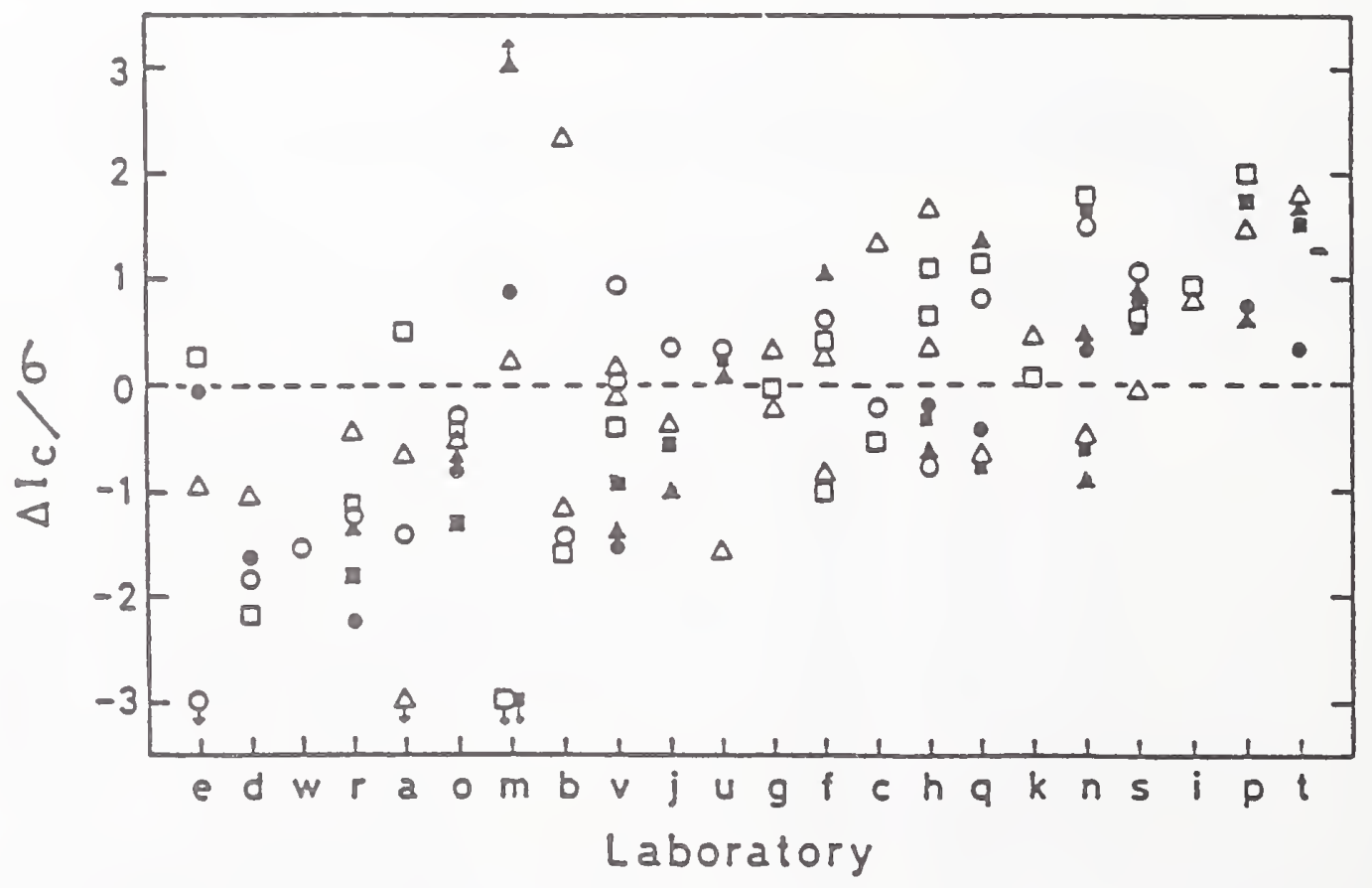

F18. 5 Comparison of I 's among part1elpant labs at 12 T. Symbols (O, 0 ), $(\Delta, \Delta)$ and $(E, D)$ refer to the samples $A, B$ and $C$ (central reaction, self reaction), respectively. 
d!fferent sapple holders, sample handling, the measuremen: me:hod, and va:1a:foos la Instrumeat callbra:10a. rore desalled papars adjzessing these possible sources of systerailc differences are planned for future publicalton.

The ratios of $I(\mid 1)$ ro I $(\mid 1)$ are shown in 518.6 for sample $B$. I $(i p)$ and $I_{c}(i b)$ denote the $I_{c}$ 's for which the self and the applied fields are in parallel and antiparallel directlons, respectively. If the specinen is elghtly flxed on the holder, there should be no difference between $I(1 i)$ and $I(t \mid)$, the rat10 of $I_{f}(11)$ to $I_{e}(11)$ betng unIty. The tightness of the flxing depends on the bonding material and 1 ts acount. Epoxy resirs such as 'stycasi' seem to give betiez fixing than grease, as 1s apparently shown 1n F1g. 6 . Some of the curves of the ra:10 I $(11) / I$ (16) drop belou unity at higher fields in Fig. 6. 'If a specimen is unifornly deformed by concentric hoop siress, the ratlo will approach unity as the fleld 1ncreases. Therefore, nonuniform deformation of specimen may resule in the rat1o less than unity.

In orfer to see if the 'self reaction' carrled out at each lab was the exact copy of the 'central reaction', data of I's for two reactions were compared. Ihis comparison betreen self and central reastions did not include all of the avallable data, but rather, 1t only included data from labs that measured both of the1z spectmens on stmilar holders. Averaged I 's of self reacted sample B are nearly the same as those of centrally reacted one. However, averaged I 's of self reacted sample A are always smaller than those of centrally reacted one, while those of self reacted sample $C$ are smaller at lowez fields and larger at higher fields than those of centrally reacted one. These results 1ndicate that I 's of samples $A$ and $C$ are more sensitive to the heat treatment condition than those of sample B. The reaction time for sample $C$ is the shortest of the three sarples and, consequently, the sensitivity of sample $C$ to both reaction tine and cemperature may be greater than for the other samples. Furthemore, sample $C$ 's apparent sensitivity to the reaction conditions may be due to an inadequate conEinement of the internal in at the specimen ends during the heat treatment. The coefficlent of variaifon of I of self reacted samples, which may reflect the difference in reaction conditions among labs, correspond to 4-8 2 of I almose 1ndependent of magnetic fleld and sample. The posslbility of sample damage incurred during shipping is also a variable in the comparison between the self and central reactions.

\section{Strain Effect}

The rensile strain effect on I's were examined at NBS, Osaka UnIv. and Rutherford lab using the round robin test samples. Although the measurement detalls were different, there was good agreement among three sets of data. As examples, resules for 14 Tesla at NBS and those for 15 Iesla at Osaka Univ. are compared in Fig. 7 where I''s have been nosmallzed to the maxisum values. The $\mathrm{I}^{\mathrm{c}}$ of $\mathrm{Nb}_{3} \mathrm{Sn}$ conducror increases with increasing tensife strain up to $0.2-0.3 \%$ where the precompressive siraln 1mposed on the $\mathrm{Nb}_{3} \mathrm{Sn}$ compound is mosily released. As can be seen in this f1gure, the rensile stain sensitivity of $I$ 1s largest for sample $C$ and relatively small for sample $B$. This may be related partly to the $\mathrm{H}$ 2 of these samples; sample $C$ has $a$ celatively low $\mathrm{f}^{2}$ of $\sim 19$ Iesla, while $1 \mathrm{is} \sim 24$ Iesla for samples $A$ and $B$. A more complete comparison of the sirain effect data will be the subject of a future paper.

Effects of the thickness of the KRP holder on the Ic were examlned at NBS $^{2}$ using spectrens of samples $B$ and

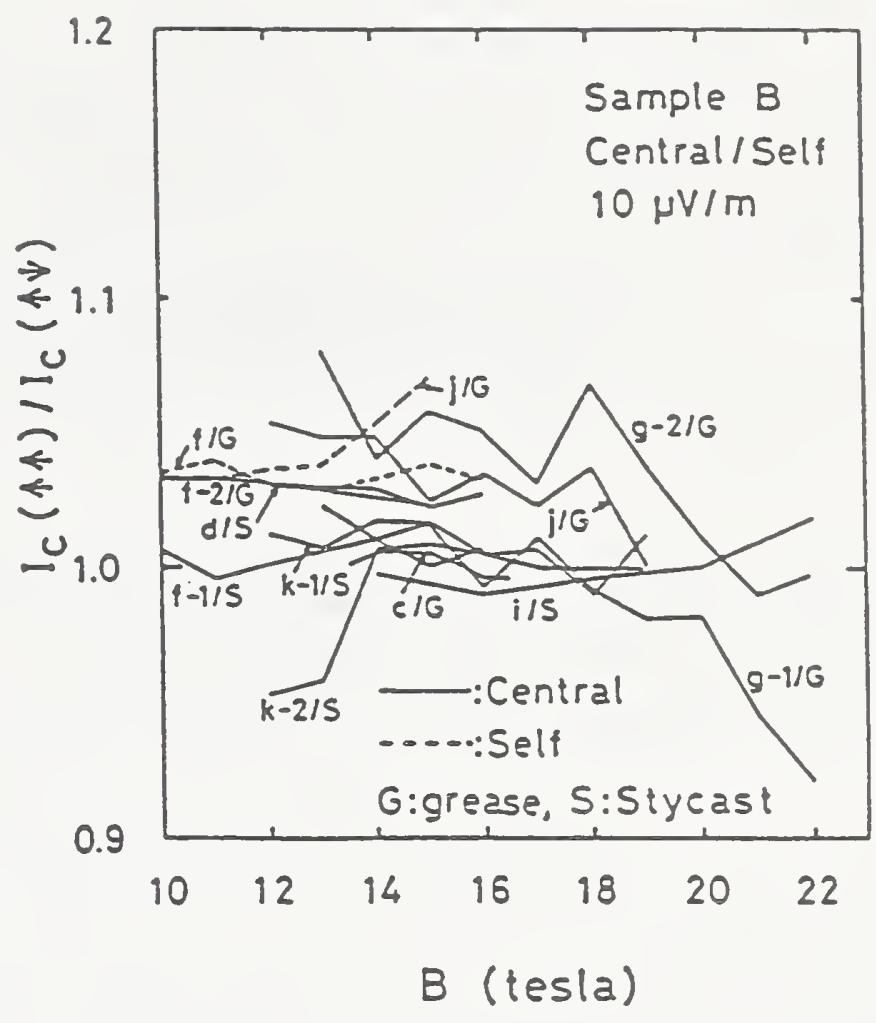

Fis.6 Ratio, $I_{e}(\mid f) / I_{e}(t h)$ vs. fleld curves for sample B.

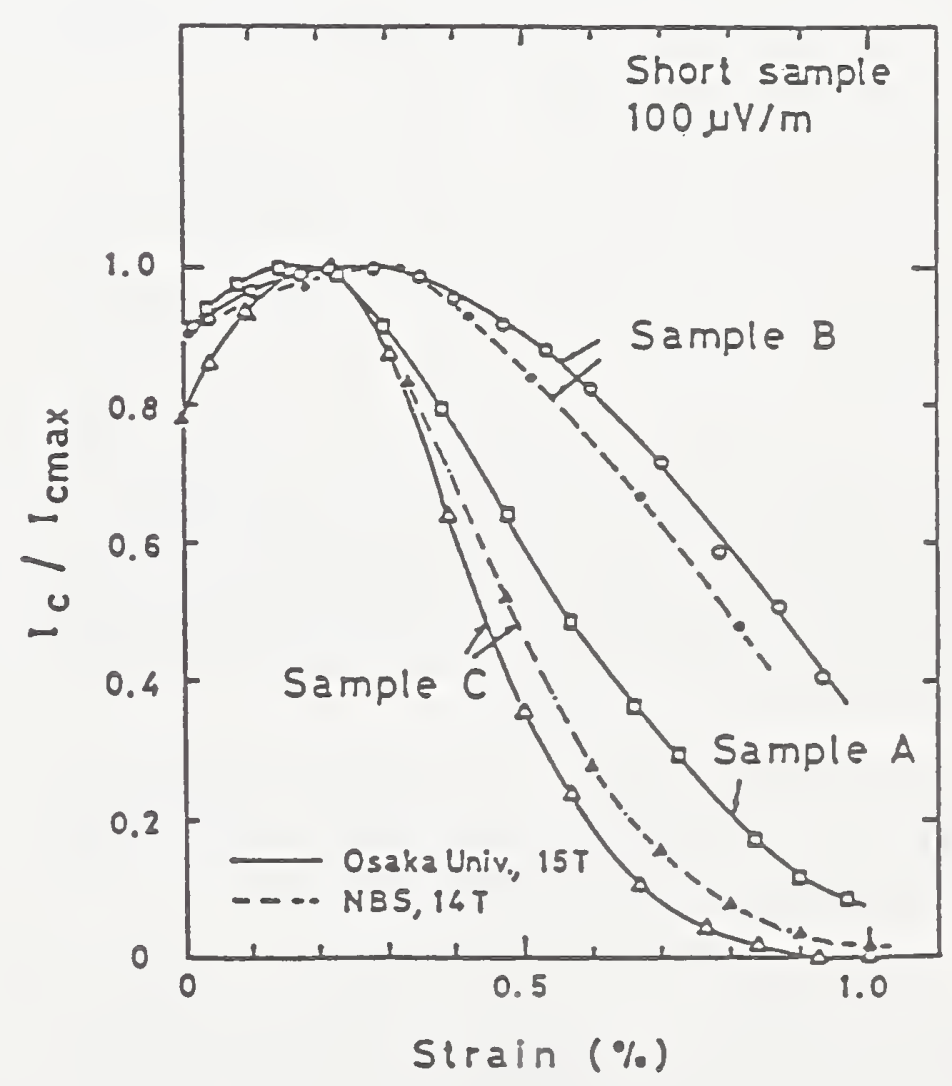

118.7 If vs. serain curves for samples $A, B$ and $C$ obiclned a NBS and Osaka UnIV. If Is normalized to Its maximum value. 
c. Specigens were flist mounted on tbick walled FRP holcers. df:er the I peasurenent the holders vere bored so as to have thin walls. It was found that I 's for these bored holders with thin walls were slightly enhanced. The themal contractions of thick and thin wall holders are different due to the textured structure of ERP, and estimated to be about $0.4 \%$ for thick wall and about $0.25 \%$ for thin wall on cooling from room temperature to $4.2 \mathrm{~K}$. It was concluded that the compressive siraln in the specinen resulting from the thermal differental coneraction between the FRP holder and the speclien was responstble for the discrepancy in I in those spectmens mounted on thick and thin wall hôlders.

The sample holder materlal and geometry, in conjunet10n with the specluen bonding method, $c$ an have an ef fect on the strain state of the specimen and this will have a signiflcant effect on the results of the $I_{c}$ measurezents. This, this could be a major source of the obsezved systematic varlation in the measured I 's.

\section{Conclusions}

Resul:s of the present round robln test may be summarlzed as follows.

a) Honogenelty test on the I showed the present samples were enough homogeneous along the whole length of wize to be used in the round robln test.

b) Coefficlents of varlarton of I 's were $7 \%, 67$ and 21: a: 12 Iesla for samples $A,{ }^{C} B$ and $C$, respect1vely. They were largest for sample $C$ whlch was most sensitive to straln and heat treatment conditlons. Coefficlent of varlation increased at higher fields for all of samples.

c) n values shored larger coefflelent of varlarlon than I. n values were racher siall in case the specinen was soldered on a werallic holder.

d) Strain effects on the I of three whes were examined. It was pointed out that the straln in the specimen was a major origin for the scatter in Ic.

e) Materlals of the specimen holder and tightness of fixlng have significant effects on the $I_{c}$ through the strain effect.

Several parameters Influencing the I value of $\mathrm{Nb}_{3} \mathrm{Sn}$ wires have been extracred and analyzed Ehrough the varas round robin rest. However, the present round robin test was perforded with few restrictions on the measurement method, and more strict determination of measurevent cond1tions will be required to winlmize the scatier in I and to furnish a really effective standard method of the $I_{c}$ measuresent.

A more complete report on the present round robin test w1ll be submitced to the steering comittee of VAYAS from the Iechnical Working Party. A rumber of papers addressing speciflc aspects of this round robin test are planned for fueure publication.

Trade names are used in this document to spectfy the measurement detalls. In no 1nstance does this 1dentiflcation 1mply endorsement by the authors or thelr Instrutions, nor does it 1mply that the particular produces are necessarily the best avallable.

The authors wish to thanix all the scientists and representaltves who have been 1nvolved in the present round robin test for thelr cooperation.

\section{References}

(1) H. Jones, "F1rst Meeting of the Vauss Iechnical Hork1ng Party on Superconductors and Cryogen1e Seructural Materlals", Cryogenles, vol. 26, pp. 488-489, August (1986).

[2] I.F. Goodr1eh and S.L. Bray, "Cr1t1cal-Current Measurements of $\mathrm{Nb}, \mathrm{Sn}$ Superconductors: NBS Coniribution to the VAMAS Interlabora:o:y Compazison", submitred for publication in Cryogenics. 
APPENDIX I

PROPOSED STUDY ON THE EFFECT OF SAMPLE BONDING TECHNIQUES ON THE MEASURED CRITICAL CURRENT OF $\mathrm{Nb}_{3} \mathrm{Sn}$ SUPERCONDUCTORS

\author{
L. F. Goodrich and S. L. Bray \\ published in: \\ Proceedings of the $6^{\text {th }}$ Japan-US Workshop \\ on \\ High-Field Superconducting Materials \\ and \\ Standards Procedures for High-Field \\ Superconducting Materials Testing. \\ Boulder, Colorado \\ February 22-24, 1989 \\ Editors: K. Tachikawa, K. Yamafuji and H. Wada \\ and \\ J.W. Ekin and M. Suenaga \\ pp. 99-108, Japan (1989)
}


Proposed Study on the Effect of Sample Bonding Techniques on the Measured Critical Current of $\mathrm{Nb}_{3} \mathrm{Sn}$ Superconductors

\author{
L. F. Goodrich and S. L. Bray
}

National Institute of Standards and Technology

Boulder, CO 80303

\title{
PREFACE
}

This proposed study was prompted, in part, by presentations that were given at the 6 th Japan-U.S. Workshop on High Field Superconductors (February 22-24, 1989 Boulder, Colorado) and subsequent discussions. The results of the recent VAMAS $\mathrm{Nb}_{3} \mathrm{Sn}$ critical-current round robin indicate that increased consistency in interlaboratory measurements might be achieved by a more detailed specification of the critical-current measurement technique. However, a competition exists between the benefit of a rigidly specified measurement technique, which insures measurement reproducibility, and a less restrictive technique that would be practical for a greater number of laboratories. Ideally, the measurement technique should have the least number of restrictions that is consistent with measurement reproducibility. This proposal addresses only one aspect of the critical-current measurement technique, the sample-to-measurement-holder bonding method. The measurement study proposed herein is typical of research that is routinely performed at NIST; however, the existing commitment of our available resources prevents us from undertaking this work now. Also, from a technical perspective, the critical-current measurement technique proposed here is not one that is normally used at NIST. Consequently, another laboratory, which normally uses a measurement technique that is similar to this one, may be better suited to this research. Dr. Hitoshi Wada of Japan's National Research Institute for Metals has expressed an interest in conducting a study of this type and this document was prepared at his request. 


\section{INTRODUCTION}

The results of the VAMAS critical-current $\left(I_{C}\right)$ round robin demonstrated the existing level of measurement discrepancy among a large number of laboratories for several different $\mathrm{Nb}_{3} \mathrm{Sn}$ samples. The discrepancy was significant. A major source of this discrepancy was determined to be varying degrees of axial sample strain caused by variations in the thermal contraction of different sample holders.

The sample bonding technique can play an important role in this effect because the strength of the bonding determines the amount of the sample holder's thermal contraction that is transmitted to the sample. A relatively weak bond can inhibit this transmission of thermal contraction but it may also allow sample motion under the influence of the Lorentz force, which, in turn, can cause variations in the measured $I_{c}$. A high strength bonding material used in conjunction with a sample holder whose thermal contraction is comparable to that of the $\mathrm{Nb}_{3} \mathrm{Sn}$ sample alleviates both of these mechanical problems but it introduces a practical problem. The commonly used sample holder material that has satisfactory thermal contraction is a fiberglassepoxy composite (G-10). The high strength bonding material that is typically used for G-10 sample holders is a filled epoxy adhesive. The problem with this method is that the epoxy forms a fairly permanent bond which makes repeated use of the sample holder difficult.

What is clearly needed is a bonding material that provides adequate sample support but that is easily removable from the sample holder. The results of the VAMAS measurements indicate that grease may be adequate for this purpose as long as all of the $I_{c}$ measurements are made with the Lorentz force directed inward, toward the sample holder. Prior to accepting grease as the preferred sample bonding material, a more definitive study of its suitability should be conducted. A general systematic method for conducting this study is proposed here.

A second $\mathrm{Nb}_{3} \mathrm{Sn} \mathrm{I}_{\mathrm{C}}$ round robin where several different measurement techniques are tested by each participating laboratory in order to determine the preferred measurement method for $\mathrm{Nb}_{3} \mathrm{Sn}$ is envisioned. This study would test a number of measurement variables including the sample holder material. Consequently, a prior determination of the preferred bonding material would help simplify this subsequent round robin. The number of laboratories participating in this second round robin might be considerably reduced, from the first round robin, because of the difficulty involved in complying with the necessarily restrictive specifications for the various measurement techniques. However, in the event that the results of the second round robin resulted in a general consensus regarding which measurement variables must be specified in order to assure consistent measurements among different laboratories, a third round robin might be useful for determining the effectiveness of the measurement method for a larger group of laboratories. 
Once again, the objective of this study is to determine the effectiveness of grease as a sample bonding material in making reproducible $I_{c}$ measurements.

For this proposed study on sample bonding, stainless steel was selected as the sample holder material, because it represents a worst case condition with respect to differential thermal contraction between the sample and its holder. In other words, if grease proves to be adequate as a bonding material on stainless steel, it should be adequate for other sample holder materials as well. Although the thermal contraction of G-10 is better matched to that of the $\mathrm{Nb}_{3} \mathrm{Sn}$ samples, there is no guarantee that G-10 will ultimately be selected as the preferred sample holder material.

Stainless steel sample holders present some measurement problems that other materials do not: The stainless steel sample holder provides a shunt path in which a portion of the metered test current may flow and, thus, it may artificially increase the measured $I_{c}$. The amount of current flowing in this shunt path depends on several variables including the joint resistance between the sample and the current contacts, the resistance between the current contacts and the sample holder, the resistance of the sample holder (which varies with the sample holder's diameter, wall thickness, and length), and the voltage across the sample. Based on the resistivity of stainless steel, an estimate of the current-contact joint resistance, the sample length, and the electric field criterion, a simple calculation can be made to estimate the maximum value of the shunt current. This calculation indicates that for most measurement conditions the shunt current is negligible in comparison with the sample current; however, in cases the applied magnetic field is high and the sample current is very low the, shunt current can be significant. Also, the stainless steel's relatively high thermal capacitance and thermal conductivity may artificially enhance the sample's stability, particularly in the case of a soldered sample. Nonetheless, stainless steel is a very commonly used sample holder material and since it seems to present the most severe test of the sample-to-holder bond, it was selected for this proposed study.

In general, the proposed method for determining the effectiveness of grease as a bonding material is to measure the $I_{c}$ of a number of $\mathrm{Nb}_{3} \mathrm{Sn}$ samples, where some of the samples are mounted to their holders using grease and some are soldered to their holders, and then compare the variation in the measured $I_{c}$ for the two sample mounting techniques. For all of the samples, the reaction and measurement variables should be held constant in order to isolate the variable of interest, sample bonding.

There are two phases in the study. The first is a necessary but insufficient test of grease's suitability as a bonding material. In this phase, all of the mounting variables should be held constant in order to insure that discrepancies in the $I_{c}$ measurements that are actually due to variations in the sample mounting technique are not attributed to inadequate sample bonding. This consideration also requires good homogeneity among the test samples. In some cases it may be advantageous to measure the same sample several times while changing the mounting technique to avoid measurement variations caused by sample inhomogeneity. The mounting technique that is 
employed for this phase of the study should be designed to optimize the grease's bonding capability. This phase of the study will thus demonstrate whether grease is an adequate bonding material under ideal conditions. If, under these ideal conditions, the grease proves inadequate in producing consistent measurement results, then it would undoubtedly be inadequate in actual practice where many different laboratories would be mounting and measuring the samples. Of course, this assumes that the mounting technique selected for this study does in fact optimize the grease's bonding capability. In a sense, the first phase of the study will test the validity of this assumption.

Assuming that the first phase of the study demonstrates that, at least under tightly controlled conditions, grease is an adequate bonding material, the second phase of the study should then be conducted. The second phase of the study addresses the fact that, in actual practice, mounting variables will exist between different measurement laboratories; to some degree there will result in variations in the $I_{c}$ measurements. The purpose of the second phase is to determine which mounting variables have a pronounced effect on the Ic measurement. The mounting variables that are purposely introduced during the second phase should be those that are believed to occur most frequently in practice. In other words, the second phase of the study should simulate the situation that occurs in an interlaboratory comparative measurement. Once these variables have been isolated, specifications for sample mounting with grease can be proposed that address these dominant variables.

In both phases of the study the soldered samples will act as a standard, of sorts, with which the grease mounted samples will be compared.

Because the solder bond is a very positive one, if the measurement variability for the grease mounted samples is comparable to that of the soldered samples, it will indicate that the strength of the grease bond is adequate. Like the grease mounted samples of the first phase, the soldered samples should be mounted with as few variables as possible to allow a legitimate comparison between the two mounting methods. For the same reason, the soldered samples of phase two should be mounted with the deliberate introduction of mounting variables, thus simulating the variability that is typical from one laboratory to another for soldered samples. The results of the phase two study will demonstrate whether the inclusion of realistic mounting variables causes greater data scatter for the grease mounted samples than for the soldered samples. The justification for conducting this study in two phases, rather than simply conducting phase two of the study, is that the second phase is considerably more difficult than the first and it need not be conducted if the first phase is unsuccessful. Equally important is the fact that phase one directly addresses the effectiveness of grease as a bonding material without the introduction of other variables.

\section{PROCEDURE}

\section{General Philosophy}

Because this study will be conducted at another laboratory, it is impractical to specify all of the details of the sample reaction, mounting, and measurement procedures. For example, a detailed specification might 
require that the sample holders have an outside diameter that is slightly different than that normally used at the testing laboratory, and compliance with this somewhat arbitrary specification would unnecessarily increase the required time and cost for this study. Consequently, the philosophy of this proposal is to state general guidelines for the study while allowing the measurement laboratory to substantially adhere to its normal practices.

\section{Sample Reaction}

The samples should be reacted on well oxidized stainless steel tubes in order to avoid diffusion bonding between the sample and the tube. When diffusion bonding occurs, the probability of damaging the sample when transferring it to the measurement holder is greatly increased. Even in the a case where diffusion bonding does not occur, extreme care must be exercised in transferring the sample to avoid damage due to bending strain. The reaction holders should have a helical groove for defining the sample coil's pitch. The sample should be well seated in this groove prior to reaction to insure reproducibility of the coil diameter from sample to sample.

\section{Sample Holders}

The stainless steel measurement holders should have the same diameter, pitch length, and groove geometry as the reaction holders to insure that the reacted sample can be fitted to the measurement holder with a minimum of sample deformation. For phase one of the study, the samples should be well seated in the measurement holder's groove to insure consistent coil diameters and, thus, consistent bending strain. This is also important for minimizing the thickness of the bonding material between the sample and holder in the case of grease mounted samples. Moreover, a thick layer of grease is more likely to yield under the influence of the Lorentz force than a thin layer, where the sample is substantially in contact with the stainless steel holder.

A good choice for the holder's groove geometry is a $60^{\circ}$ "V" with a fairly sharp point. The $60^{\circ}$ groove provides good sample support in both the radial and transverse directions. The sharp point of the groove insures that the conductor contacts the groove at the points of tangency rather than at the bottom of the groove. In the case of the measurement holder, this should minimize the thickness of the bonding-material layer between the sample and the holder. In the case of the reaction holder, it may reduce diffusion bonding. The groove should be deep enough that the sample contacts the holder within the groove rather than at the interface formed between the groove and the holder's surface. This is important for transverse stability of the sample. One detrimental consequence that results from making the groove too deep is that the sample will require greater deformation during the transfer process in order to clear the holder.

\section{Sample Mounting}

For the grease mounted samples, the measurement holder should be prepared by filling the sample groove with grease, avoiding any excess grease outside the groove. This procedure insures that equal amounts of grease are applied to all of the samples and that there is not an excess of grease, 
that might inhibit cooling. Since all of the measurements will be made with the Lorentz force directed inward, there is limited benefit in having excess grease on top of the sample.

Before attempting to transfer the sample from the reaction holder, it should be checked for diffusion bonding to the holder. If there is a significant amount of bonding, that might result in sample damage, the sample should be discarded. There are two methods that can be used for removing the sample from the reaction holder. The first method requires a very smooth interface between the sample and holder but, when it can be employed, it allows sample removal with minimal deformation. In this method the sample is unthreaded from the holder by lightly gripping the sample coil's surface while turning the holder. Alternatively, the sample coil's diameter can be expanded by carefully twisting the coil until the holder can be extracted.

Although this second technique results in some sample deformation, it is the same deformation that must be introduced when the sample is mounted on the measurement holder. In the case of the grease mounted samples, the sample cannot be threaded on to the measurement holder because the grease must be applied to the sample groove prior to mounting the sample. Although the soldered samples could be threaded onto the measurement holder prior to soldering them in position, this would introduce an undesirable variable between the soldered samples and the grease mounted samples. Consequently, both the soldered and the grease mounted samples should be transferred using the same technique.

Before soldering the grease mounted samples to both of their current contacts, the slack in the sample coil should be removed by firmly seating it in the sample groove. This is easily achieved by first soldering one end of the sample coil to its current contact and then, starting at that contact, pressing the sample into its groove in a continuous motion that results in all of the coil's slack being transferred to the opposite current contact. The sample can then be fixed in this taut position by soldering it to the current contact. The grease that is pressed out of the groove along the sample's sides during this operation increases its transverse stability and should not be removed.

In the case of the solder mounted samples, slack should be removed prior to soldering and then mechanical retainers should be used at both ends of the sample to hold it in the taught position during the soldering operation. To insure reproducibility, the ends of the sample should not be allowed to move during soldering. The sample holders should be pre-tinned prior to mounting the sample to simplify the soldering operation and to, thus, reduce the probability of sample damage.

An attempt to consistently regulate the shunt current that flows in the stainless steel holder should be made. If the current contacts are electrically isolated from the sample holder (with the exception of the sample current path), the shunt current should be fairly consistent from sample to sample. However, if the contacts are not isolated, then the shunt current will depend on, among other factors, the resistance of the solder joints at the current contacts and the resistance of the interface between 
the contacts and the holder. Consequently, the length of the contact joint should be the same for all of the samples and the resistance of the contactto-holder interface should be constant.

\section{Current Reversal}

Grease will undoubtedly present a limitation in the $I_{c}$ measurements that other more permanent bonding materials do not. Its relatively low tensile strength will probably disallow its effective use for $I_{c}$ measurements where the Lorentz force on the sample coil is directed outward. This, in turn, disallows sample current reversal for testing the effect of the sample coils self-generated magnetic field on the measured $I_{c}$.

\section{Statistics, Field History, and Repeat Determinations}

The number of each sample type, grease mounted or soldered, that need be measured for this study cannot be specified beforehand. It is important that the number of measurements is large enough to be statistically significant, but the number of measurements required to meet this criterion depends on the observed measurement variability for each of the sample types. Because the Lorentz force may play an important role in the $I_{c}$ measurements of the grease mounted samples, the magnetic field history of the test sample may effect the measurements. For example, if the first $I_{c}$ measurement is made at the field where the Lorentz force on the sample is a maximum, the subsequent measurements might be different than for another field sequence. Consequently, the measurement sequence should be carefully considered and recorded. Also, if the Lorentz force causes irreversible sample motion, subsequent measurements at the same magnetic field may have different results. Repeat determinations of the $I_{c}$ should be used to detect mechanical deterioration of the sample bond.

\section{Grease Mounting Variables}

The first variation on the phase-one measurements that should be studied is the effect of eliminating the bonding material altogether the effect of the bonding material can be isolated. By measuring the $\mathrm{I}_{c}$ of several samples that are tightly mounted on grooved holders without any bonding material and comparing the resultant measurement variability with that of bonded samples.

In the second phase of the study, common mounting variables should be introduced to the measurements. For the grease mounted samples, a common variable might be the relative tightness of the sample coil on the measurement holder. This variable is important for two reasons. First, the level of bending strain in the sample will depend on the coil diameter and, thus, it will depend on how closely the sample is fit to the holder. Also, the thickness of the grease between the sample and the holder will vary with the sample tightness. This might lead to variations in the rigidity of the sample under the influence of the Lorentz force.

The difficulty in studying the effect of this variable is in measuring the sample tightness. One method for quantifying the sample tightness is to place the sample on the holder, without using any grease, mark the position of 
the sample ends on the holder, remove the sample from the holder, apply grease to the holder, remount the sample in a slack position, and measure the distance from the ends of the sample to the marks on the holder. This technique should result in a reproducible level of slack in the sample coil. It is important not to tighten the sample beyond the position in which it will ultimately be tested because some of the grease may be irreversibly extruded from the groove and the bond strength might suffer. By measuring the $I_{c}$ as a function of sample tightness the sensitivity of these measurements to the grease layer thickness and the sample bending strain can be studied.

Another mounting variable that exists at different laboratories is the geometry of the sample holder groove. This geometry may effect the mechanical stability of the grease mounted samples when under the influence of the Lorentz force. It is impractical at this point to measure the effect of a wide range of different groove geometries on the measured $I_{c}$; however, there is a limiting case that should be measured. A holder having no groove at all should represent the lower limit of transverse sample stability. By studying this extreme case the importance of providing transverse stability, by means of a sample groove, can be determined. In preparing a smooth sample holder for this study it is important to make its outside diameter such that the resulting diameter of the mounted sample coil is equal to that of the phaseone samples that are mounted on grooved holders. This will assure that the bending strain is not an inadvertent additional variable in this study.

In the phase-one measurements, it is specified that all the mounting grease be applied to the sample groove before transferring the sample to the measurement holder, thus controlling the quantity and position of the grease. In practice, the top of the sample is often coated with grease after it is transferred to the measurement holder. This procedure may effect the measured $I_{c}$ in several ways. The additional grease may increase the mechanical coupling between the sample and holder. Consequently, more of the differential thermal contraction between the sample and holder may be transmitted to the sample, thus increasing the level of compressive strain. The increased sample-to-holder coupling may also make the sample more stable under the influence of the Lorentz force. Finally, if a heavy overlayment of grease is applied to the sample it may inhibit cooling and reduce the measured $I_{c}$. To test these effects, samples should be measured with several levels of grease overlayment.

Different laboratories most likely cool their samples at significantly different rates; in the limiting cases, some may slowly cool the sample in helium vapor while others may insert the sample directly in a liquid helium bath (or perhaps liquid nitrogen). This variable may affect the measured $I_{C}$ of grease mounted samples. The grease bonding material has virtually no mechanical strength until it is cooled below its freezing temperature. Consequently, the strength of the coupling between the sample and its holder is changing during the cooling process while differential thermal contraction between the sample and holder is occurring. This fact may result in different mechanical states for samples cooled at different rates. If this variable does have an effect on the measured $I_{c}$, it is probably greatest when a thick overlayment of grease is applied to the sample. Aside from providing a greater potential for a strong coupling between the sample and holder, a thick 
layer of grease also creates a thermal insulation layer between the holder and the helium bath that might increase the difference in the cooling rates for the various components of the sample-holder assembly. To test the effect of the sample cooling rate, at least two samples should be measured; both should have a thick grease overlayment and one should be slowly cooled while the other should be inserted directly in a liquid helium bath.

Grease is a generic term that applies to a very broad range of heavy lubricants that certainly have significantly different physical properties. Two such materials that have been used for sample bonding materials are petroleum jelly and vacuum grease. Undoubtedly, other materials have been, or might be, used for this purpose. The variation in their physical properties might be a significant interlaboratory measurement variable. To test the effect of this variable on the $I_{c}$ measurement, a representative group of greases that are used for sample mounting should be assembled and comparative $I_{c}$ measurements should be made using these various greases.

\section{Solder Mounting Variables}

The significant mounting variables associated with soldered samples are presumably fewer than those for grease mounted samples. This is one of the benefits of using soldered samples as the comparative standard for this study. However, to legitimately compare soldered and grease mounted samples in the second phase of this study, it is necessary to simulate the typical variability found in soldered samples that are prepared at different laboratories. Assuming that the soldered samples are carefully handled to avoid damage, the dominant mounting variable is probably the strain state of the conductor, which may vary depending on the soldering technique that is employed.

The technique that has been described for testing the effect of sampleto-holder tightness for grease mounted samples is also applicable to soldered samples. Since the thickness of the solder layer between the sample and holder is probably of no consequence, this test would simply measure the effect of varying the sample's bending strain. Another soldering technique that may be used and that might result in a different strain state for the sample is to simply start at one end of the sample and solder it along its length without first anchoring its ends to the current contacts. Several samples should be prepared and measured using different soldering techniques in order to test the variation of solder mounted samples.

\section{Concluding Remarks}

This document provides general guidelines for studying the suitability of grease as a bonding material in $\mathrm{Nb}_{3} \mathrm{Sn} \mathrm{I}_{c}$ measurements. The details of the study will undoubtedly require adjustments based on intermediate results. For example, if the initial phase-one measurements are not adequately consistent, the sample mounting technique will require adjustment and further testing before the second phase of the study can begin. In the event that these measurements indicate that grease is an adequate bonding material for the particular conductor and sample holder that is tested, this does not mean that it will be adequate for all conductors or sample holders. Larger conductors 
will be subjected to greater Lorentz forces and the grease may be incapable of constraining these forces. Also, the grease's adhesion to other sample holder materials may be different than it is for stainless steel. Finally, the second phase of this study is important in learning about the effect of various mounting variables but it cannot be fully conclusive because it will not address all of the possible interlaboratory variables. However, presuming that the phase one measurements are satisfactory, the mounting technique could be tightly specified to insure measurement consistency. 



\section{APPENDIX J}

CURRENT RIPPLE EFFECT ON SUPERCONDUCTIVE d.c. CRITICAL CURRENT MEASUREMENTS

L. F. Goodrich and S. L. Bray

Cryogenics 28, pp. $737-743$ (1988) 


\title{
Current ripple effect on superconductive d.c. critical current measurements*
}

\author{
L. F. Goodrich and S. L. Bray \\ Electromagnetic Technology Division, National Bureau of Standards, Boulder, C0 80303 , \\ USA
}

Received 10 May 1988

\begin{abstract}
The effect of current ripple or noise on d.c. critical current measurements was systematically studied. Measurements were made on multifilamentary Nb-Ti superconductor. A low-noise, battery-powered current supply was required in this study in order to make the pure d.c. critical current measurements. Also, an electronic circuit that stimulates a superconductor's general current-voltage characteristic was developed and used as an analysis tool. In order to make critical current measurements in which current ripple was present, the battery supply was modified to allow the introduction of controlled amounts of a.c. ripple. In general, ripple in a current supply becomes more significant in current supplies rated above $500 \mathrm{~A}$ because effective filtering is difficult. The effect of current ripple is a reduction in the measured d.c. critical current; however, ripple of sufficient amplitude can result in arbitrary measurement results. The results of this work are general and quantitatively applicable to the evaluation of critical current data and measurement systems. A theoretical model was developed to further support and explain the ripple effect. An unexpected benefit of this work was a more precise method for general critical current data acquisition. Problems common to all large conductor critical current measurements are discussed.
\end{abstract}

Keywords: critical currents; superconductors; $\mathrm{Nb}-\mathrm{Ti}$

The presence of current ripple (any periodic departure from d.c. output level) reduces the measured d.c. critical current, $I_{\mathrm{c}}$ (see Reference 1). In addition to quantifying this effect, an attempt was made to determine its source. Several possibilities are discussed. A recent literature search indicates that this is the first documented study of this type. The $I_{c}$ measurements reported here were made using a straight sample geometry and a radialaccess magnet.

\section{Experimental apparatus and procedure}

\section{Battery powered current supply}

A batiery current supply was modified to allow the creation of current ripple with variable frequency and amplitude 2 . The maximum d.c. output of this supply is $1000 \mathrm{~A}$. The maximum tested frequency, a.c. amplitude, and d.c. bias for this supply are $10 \mathrm{kHz}, 170 \mathrm{~A}$, and $400 \mathrm{~A}$, respectively (these values were not achieved simultaneously). The performance of the supply as a source of a.c. current at several frequencies was evaluated by measuring the peak amplitude and RMS values of the output current. To judge the shapes of the waveform, the ratio of the pcak amplitude to the RMS value was compared to thc theoretical value for the given waveform (1.73 for a

* Official contribution of the National Bureau of Standards, not subject 10 copyright triangular wave and 1.41 for a sinusoid). For both waveforms and all frequencies $(60 \mathrm{~Hz}$ triangle; 60,360 , and $1000 \mathrm{~Hz}$ sinusoids) the variation between the theoretical and measured ratios increased with increasing a.c. amplitude. For $I_{c}$ measurements at $8 \mathrm{~T}$, this variation was nearly independent of frequency and had a maximum value of less than $12 \%$ at the maximum tested a.c. amplitude of $70 \mathrm{~A}$. At amplitudes below $40 \mathrm{~A}$, the maximum variation was less than $5 \%$.

\section{Sample and instrumentation}

The sample measured in this experiment was a commercial multifilamentary $\mathrm{Nb}-\mathrm{Ti}$ superconductor with a diameter of $0.65 \mathrm{~mm}$. The filament twist pitch was 0.8 tu'ist $\mathrm{cm}^{-1}$. There were $6006,5 \mu \mathrm{m}$ diameter filaments with a Nb diffusion barrier around each of the filaments. The sample w'as contacted with several sets of twisted-pair voltage taps. This allowed simultaneous acquisition of data with several voltmeters to deiermine any variation in the observed effect, any difference in voltmeters, and any effect due to voltage-tap geometry. One voltage-tap pair was connected to the sample in such a way that the mutual inductance bctween the voltagc-lap pair and sample was enhanced. It spanned the same segment of the sample as another lowcr mutual-inductance pair. Two other voltage-tap pairs had their wircs connected ncar the samplc, and only onc of the wires was connected to the sample (referred to as null tap pairs). This provided 
pairs that should not have any non-inductive voltagc. One of these pairs had low and the other had high mutual inductance. This allowed for a determination of how the voltmeters measure a purcly inductive voltage.

The sample voltage has scveral components. For this work, the intrinsic d.c. transport $V-I$ characteristic of the superconductor was assumed to have the voltage proportional to the current raised to the power $n$, where $n$ for the tested sample was about 30 (see Reference 1). There might be a slight current transfer voltage but otherwise the non-inductive component of the voltage should be zero, within measurement limits, until the current approaches the $I_{\mathrm{c}}$. Here, the highly current dependent flux-flow voltage occurs. The unavoidable loop made by the pair of voltage taps and the segment of the sample between them, will give an inductive voltage proportional to the time derivative of the sample current. There will be a highly asymmetric voltage signal due to periodic entry of the peak current into the flux-flow region of the superconductor at high enough bias currents.

\section{Superconductor simulator}

A simple electronic circuit was designed to simulate the intrinsic $V-I$ characteristic of a superconductor. This circuit was used to characterize the response of a nanovoltmeter when the input voltage is a highly asymmetric periodic voltage which results from passing a d.c.-biased a.c. current through a superconductor. A more general application of this circuit had been to aid in the development and testing of critical current data acquisition systems. This circuit was developed based on an idea by Ekin and Brauch of NBS. The original idea used a diode in series with a current sensing resistor. The combination was placed in parallel with a high current shunt resistor. This passive circuit gives a non-linear $V-I$ characteristic. Unfortunately, the curve is not very sharp $(n \approx 10)$ and it is not adjustable. An active simulator circuit is shown in Figure 1. The input of the simulator circuit comes from a large shunt connected in series with the current supply. This input voltage is compared with

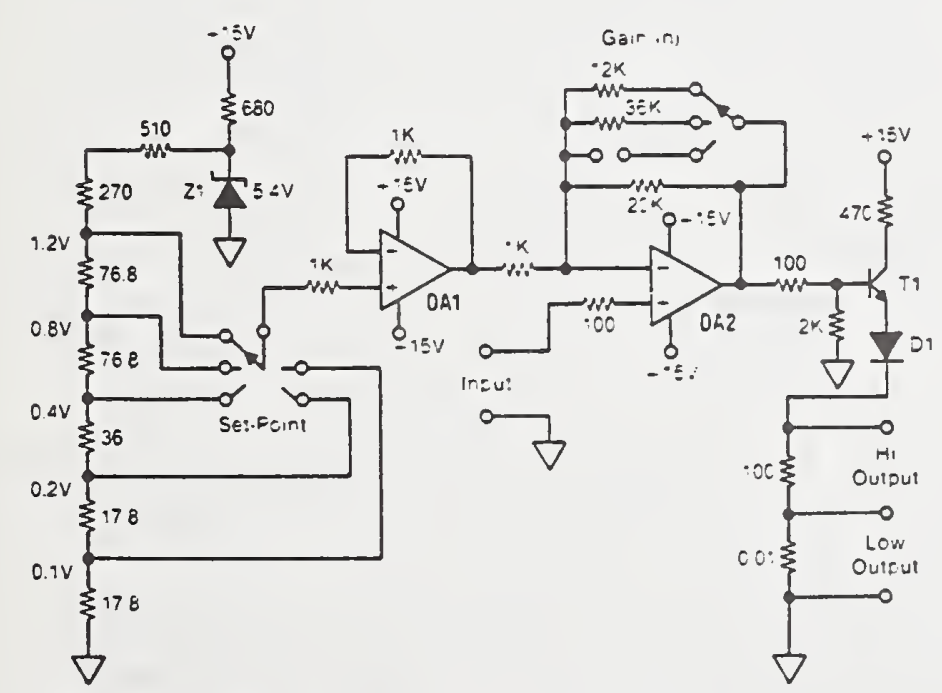

Figure 1 Schematic diagram of an electronic circuit that simulares the intrinsic voltage current characteristic of a superconductor a bias voltage and then amplified by OA2. The adjustable bias voltage produced by OA 1 simulates the $I_{c}$. No output occurs until the input voltage has risen above the bias voltage. The gain of the amplifier may be varied and simulates the $n$ of a superconductor. The voltage output of this amplifier stage drives the base of transistor T $\mathrm{i}$. A non-linear current results at the emitter of the transistor. This non-linear current results from the combination of the diode (D1) and output transistor (T1). The current passes through two shunt resistors that provide the circuit's output signals. Typically, the nanovoltmeter to be tested is connected to the $0.01 \Omega$ resistor and a recording instrument is connected to the $100 \Omega$ resistor. Another channel of the recording instrument is connected to the analog output of the nanovoltmeter. These two channels are then compared and the nanovoltmeter may be thus characterized under conditions similar to an $I_{c}$ measurement. The $0.01 \Omega$ series resistor is externally connected to the remainder of the simulator circuit to allow thermal stabilization of the nanovolimeter to resistor connection.

\section{Data acquisition}

Two types of voltmeter were used to measure the sample voltage. One was an analog nanoroltmeter and the other was a digital nanovoltmeter. Both were direct current meters with an amplified analog output, which was fed to a digital processing oscilloscope. The voltage signal of a current sensing resistor was split, amplified, and fed into two channels of the digital processing oscilloscope. One of these current signals was filtered to give the d.c. component and the other was unfiltered (passed wide band). A total of eight channels (two current and six voltage) were used to acquire data simultaneously.

The sample voltage and current were measured at relatively fixed current ripple and many d.c. bias currents (point method). These points along the voltage-current $(V-I)$ curve were analysed to determine the $I_{c}$. This procedure is different from the more typical procedure where the current is ramped continuously with time while the voltage and current are recorded. Ramping the current was not an option in this experiment because the a.c. and d.c. components of the current had to be accurately separated and the steady state voltage measured. The point method has some general advantages over the ramp method. The point method allow's for a longer time averaged voltage to be measured (if voltage noise is a problem) without greatly increasing the time required for taking a complete $V-I$ curve. This is achieved by selecting a distribution of current bias points that results in a more precise measurement. This requires taking a few widely spaced points in the low current portion of the curve to establish the base line and then taking a progressively closer-spaced point distribution as the current increases toward $I_{c}$. The point method greatly reduces the effect of inductive voltage and sample motion. The only disadvantages are that it takes longer because the voltage has to settle for each data point and the general shape of the curve has to be known a priori. The $I_{c}$ was defined as the d.c. current at which the measured electric field strength rose to $0.2 \mu \mathrm{V} \mathrm{cm}^{-1}$. The precicion of the $I_{c}$ measurement was about $\pm 1 "$ '" 


\section{Results}

\section{Voltmeter tests}

In order to develop a quantitative understanding of the nanovoltmeter's input-output relationship for a.c. voltage inputs and thus determine the effect on the measured $I_{c}$, two a.c. tests were conducted on both the digital and analog nanovoltmeters. The first test (symmetric test) consisted of applying sinusoidal voltage signals of various amplitudes and frequencies to the nanovoltmeter input and measuring the resulting output signal. A.c. signals of appropriate amplitudes were obtained by dividing the output voltage of an a.c. signal generator. The objective of the second test (asymmetric test) was to determine the response of the nanovoltmeters to the highly asymmetric a.c. voltage that is characteristic of a superconductor carrying a sinusoidal current. The superconductor simulator circuit was employed to generate the required asymmetric nanovoltmeter input signals.

The results of the symmetric tests were considerably different for the digital and analog meters. In both cases, the d.c. output of the meters increased with increasing a.c. input amplitude. However, the output signal from the analog meter was approximately an order of magnitude greater than that of the digital meter for a given a.c. input. Defining the a.c. rejection (ACR) of the nanovoltmeter as 1 minus the ratio of the d.c. output voltage to the peak a.c. input voltage provides a useful measure of a meter's relative tolerance of a.c. input voltages. This is not the common mode rejection ratio $(C M R R)^{3}$. For the analog meter, a $60 \mathrm{~Hz}, 500 \mu \mathrm{V}$ peak a.c. signal resulted in a d.c. output of $5.2 \mu \mathrm{V}$, or an ACR of $99.0 \%$. The same input signal applied to the digital meter resulted in a d.c. output of $0.66 \mu \mathrm{V}$, or an ACR of $99.9 \%$. This input signal was on the high end of those encountered in this ripple experiment, indicating that a detectable artificial voltage may be observed for the analog meter. For a voltage-tap pair with a $1 \mathrm{~cm}$ separation, a typical mutual inductance was about $2 \mathrm{nH}(53 \mu \mathrm{V}$ peak for $60 \mathrm{~Hz}$ and $70 \mathrm{~A}$ amplitude; $880 \mu \mathrm{V}$ peak for $1000 \mathrm{~Hz}$ and $70 \mathrm{~A}$ amplitude). The mutual inductance of the high inductance taps was about $6 \mathrm{nH}$.

The dependence of the d.c. output on the input signal's frequency $(60,360$, and $1000 \mathrm{~Hz})$ was also investigated. Again, there was a significant difference between the response of the analog and digital meters. Although the d.c output of both meters did not show monotonic dependence on the input signal's frequency, the analog meter showed a considerably stronger frequency dependence. For the analog meter, a $250 \mu \mathrm{V}$ peak a.c. signal at 60,360 , and $1000 \mathrm{~Hz}$ resulted in a d.c output of 0.8 , 3.5 , and $0.5 \mu \mathrm{V}$, respectively. The same input signals applied to the digital meter resulted in a d.c. output of $0.14,0.16$, and $0.10 \mu \mathrm{V}$, respectively.

In the case of the asymmetric test, the behaviour of the two meters was essentially the same. A broad range of d.c. biased sinusoidal signals was applied to the input of the simulator circuit resulting in a large variety of typical asymmetric nanovoltmeter input signals. As before, three a.c. frequencies $(60,360$, and $1000 \mathrm{~Hz})$ were tesied. In order to determine the nanovoltmeters' responses to these input signals, the signals were observed and analyses on a digital processing oscilloscope. The peak-to-peak, timeaveraged, and RMS values of the input signals were thus determined and compared with the output of the nanovoltmeters. The results were that, regardless of the meter being tested or the character of the input signal (frequency, d.c. bias level, and a.c. amplitude), the nanovolt meter's output signal was always within $5 \%$ of the time-averaged (d.c.) value of its input signal. In other words, the nanovoltmeter behaves like a very low frequency, low-pass filter, which results in an output from the nanovoltmeter that is a time average of its input signal. This behaviour is consistent with the symmetric test where the meters were show'n to measure the time average of their input signals.

\section{Theoretical results}

The motivation for modelling this effect is to help understand what is being measured and how to generalize these results to different samples, ripple, and voltmeters. It will become clear that the shape of the $V-I$ curve and the amplitude of the ripple will change the magnitude of this effect. To extend the d.c. $I_{c}$ criterion to a general current waveform in a way consistent with the design limits of a superconductor application, the measured voltage criterion should reflect the ohmic pow'er loss within the superconductor. Differentiation between voltage and electric field strength criteria will be dropped in this section.

It is instructive to consider the power dissipated in a normal conductor when the conductor is carrying d.c.biased a.c. current. A normal conductor is similar to a superconductor with an $n$ of 1 and, thus, would have a finite critical current, based on a voltage criterion. The magnitude of the effect of a.c. ripple on the measurement of d.c. $I_{c}$ can be calculated for this case. For the purpose of this argument, start with the definition of $I_{c}$ as the RMS value of the total current (a.c. and d.c.) at which the RMS of the total voltage equals some criterion. This would be correct for a normal, ohmic sample because it is analogous to the derivation of a.c. power. For sinusoidal waveforms, the time average of the instantaneous power is the product of the RMS of the current and the RMS of the resistive voltage. Hou'ever, measurements of this voltage would require a complex voltmeter that could separate the resistive from the inductive voltages and be capable of measuring the RMS of the total (a.c. and d.c.) resistive voltage. Based on this definition of $I_{c}$ and value of $n$ equal to 1 , the measured d.c. $I_{c}$ would be equal to the square root of: the $I_{\mathrm{c}}$ without ripple squared minus the RMS of the ripple squared. For example, the power of $99.9 \mathrm{~A}$ d.c. plus $5 \mathrm{~A}$ RMS a.c. is equal to the power of $100 \mathrm{~A}$ d.c. $\left[99.9=\left(\left(100^{2}-(5)^{2}\right)^{\frac{1}{2}}\right]\right.$. The two components of current add in quadrature, thus for a low percentage ripple the effect on the d.c value is very small, for $n=1$.

A d.c. voltmeter will measure the time av'erage of the voltage, which is close to the correct quantity to measure for a superconductor with finite $n$. Consider the case where the d.c. bias current is well below the flux-flow region and the a.c. amplitude is just large enough to put the total current into the flux-flow region. The instantaneous voltage signal will be zero except at the peak of the current when it will look like a periodic delta function. Considering the a.c. power analogy, the current is fairly constant for the period of non-zero voltage and the time average power becomes the product of the time alcrage of the tu.o. Thus the d.c. voltmeter is the preper instru- 
ment to use based on the definition of $I_{\mathrm{c}}$ that relates to the average power. It can be shown that for $n=2$ and a sinusoidal waveform, the time average of the voltage gives the same d.c. value of $I_{c}$ as the time-averaged power for the case where $n=1$. In general, the difference between the time-averaged voltage and the time average power is a difference of 1 in the exponent. For an $n$ of infinity, the d.c. voltmeter will give a reduction in the measured $I_{\mathrm{c}}$ that is equal to the a.c. amplitude. This sets the limits for the effect and these are consistent with the experimental data. Current ripple should be considered as a percentage of $I_{\mathrm{c}}$ and this in combination with $n$ can be used to estimate the effect.

Calculating the time average of the instantaneous, resistive voltage would model the measurement of d.c. critical current with ripple. This can be done by starting with the approximate voltage-current relationship.

$V=V_{0}\left(I_{i} I_{0}\right)^{n}$

where: $I_{0}$ is a reference critical current at a voltage criterion of $V_{0}: V$ is the instantaneous voltage: $I$ is the instantaneous current: and $n$ reflects the shape of the curve with typical values from 20 to 60 (a higher number is a sharper transition). The time average of this voltage, $V_{\mathrm{ta}}$, is

$V_{\mathrm{La}}=\left[V_{\mathrm{o}} /\left(I_{\mathrm{o}}\right)^{\mathrm{n}}\right] \int_{0}^{1} I^{n} \mathrm{~d} t$

where the period was set to $1 \mathrm{~s}\left(\omega=2 \pi \mathrm{s}^{-1}\right)$ in general. For a sinusoidal and d.c. current, the instantaneous current can be expressed as

$I^{n}=[i+a \sin (2 \pi t)]^{n}=i^{n}[1+(a / i) \sin (2 \pi t)]^{n}$

where $i$ is the d.c. component of the current and $a$ is the amplitude of the sinusoidal component. Thus, the timeaveraged voltage becomes

$V_{\mathrm{ta}}=V_{0}\left[\mathrm{i} / I_{0}\right]^{n} \int_{0}^{1}[1+(a / i) \sin (2 \pi t)]^{n} \mathrm{~d} t$

or

$$
V_{\mathrm{a}}=V_{0}\left[i i_{0}\right]^{n} G
$$

where $G$ is defined as the value of the integral and $V_{\mathrm{ta}}=V_{\mathrm{o}}$ when $I_{0}^{n}=i^{n} G$. Also, if a d.c. instrument is used to measure the sample current, $i$ will always be the measured quantity regardless of the a.c. and the d.c. current levels. Based on these definitions, $I_{0}$ is the d.c. critical current without ripple and the value of $i$, where the sample voltage equals the $I_{c}$ criterion, is the measured d.c. critical current with ripple present, $i_{c}$. In order to keep the equations based on measured quantities instead of theoretical quantities, such as $I_{0}$, the percent difference, $D$, is defined as the difference between $I_{0}$ and $i_{c}$ normalized to $i_{c}$

$D=100\left(I_{\mathrm{o}}-i_{\mathrm{c}}\right) / i_{\mathrm{c}}=100\left(G^{[1 / \mathrm{n}]}-1\right)$

This definition is consistent with $a / i_{\mathrm{c}}$ ratio in the integral (the ripple is relative to the measured quantity $i_{c}$ ).

The integral $G$ was determined by numerical integration for a number of values of $n$ and $a / i_{c}$ ratios. These results are shown for two different ranges of current ripple in Figures 2 and 3. The $x$ axis is percent ripple amplitude (percent of $a / i_{\mathrm{s}}$ ) and the $y$ axis is the percent reduction in measured critical current. $D$. The line of $I_{\mathrm{c}}$ reduction equal to the a.c. amplitude was predicted as the limit as

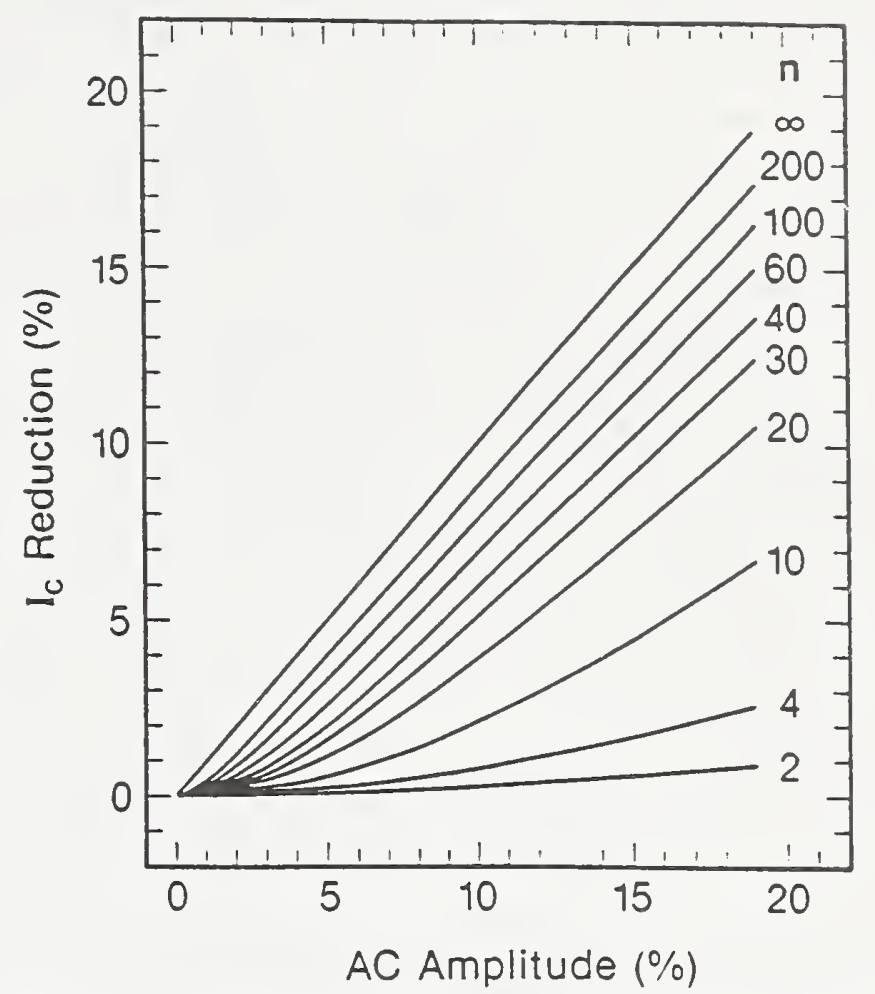

Figure 2 Model curves of the percent $I_{c}$ reduction versus the percent a.c. amplitude for several values of $n$

$n$ approaches infinity, and these plots are consistent with this limit. Consider the physical meaning of a point on this limit line where the ripple amplitude is $100 \%$; at this point, the $I_{\mathrm{c}}$ reduction is $100 \%$. The amplitude of the ripple is equal to the measured d.c. critical current with ripple present $\left(a=i_{c}\right)$ and $I_{o}=2 i_{c}$.

\section{Comparison on model and experimental data}

The $I_{\mathrm{c}}$ for increasing d.c. bias current was measured as a function of a.c. amplitude and frequency using the two

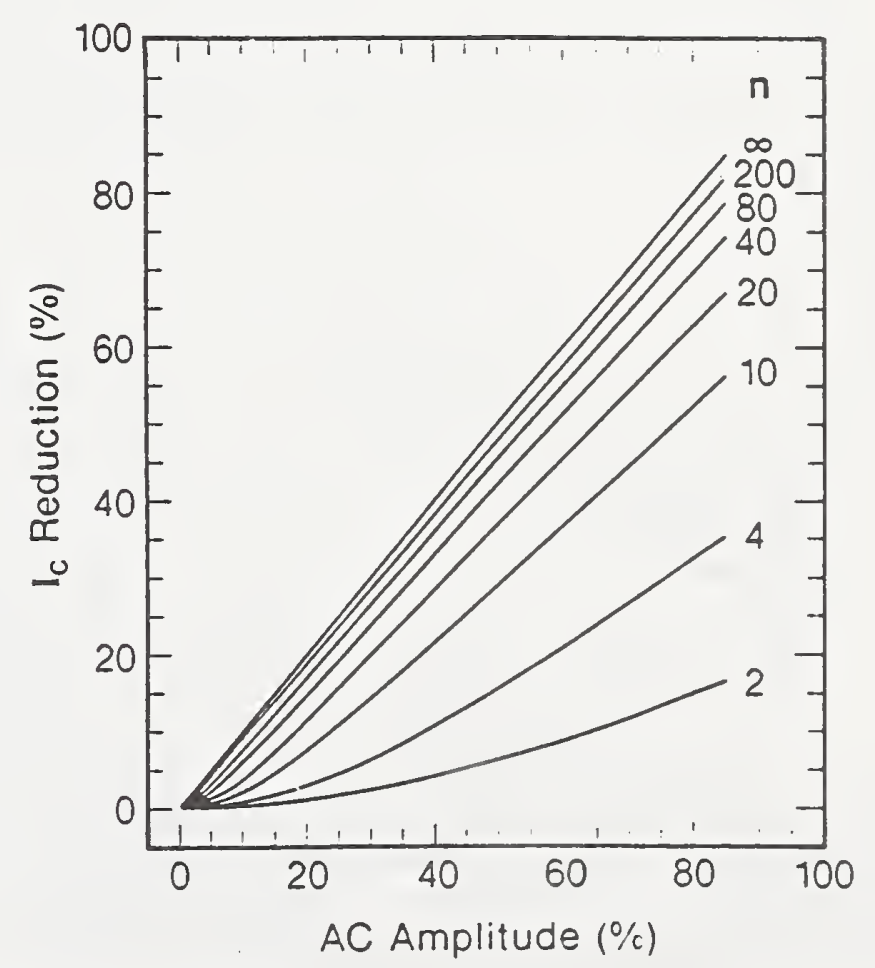

Figure 3 Model curves of the percent /c reducticn versus the percent a.c. amplitude for several values of $n$ 
different types of volimeters and various pairs of voltage taps. The reduction in the measured d.c. $I_{c}$ versus a.c. amplitude is shown in Figures 4, 5,6 and 7. These data were taken with a magnetic field of $8 \mathrm{~T}$ where the $I_{c}$ without ripple was $140 \mathrm{~A}$. The symbols indicate actual data points and the solid lines are the model curves. The a.c. amplitude is the peak value of the a.c. component of the current waveform (not peak-to-peak or peak of total current). A reference line $(n=\infty)$ was drawn for an $I_{c}$ reduction equal to the a.c. amplitude. For all frequencies and waveforms, these data are just below this reference line indicating that the effect is to reduce the measured $I_{c}$ by slightly less than the a.c. amplitude. The $-60 \mathrm{~Hz}$ frequency was labelled negative to indicate that it was a triangular waveform at $60 \mathrm{~Hz}$ rather than a sinusoidal waveform. At all of the other frequencies, the waveform was sinusoidal with very little distortion. The zero frequency data had only the intrinsic ripple of the supply, which was about $0.3 \mathrm{~A}$ in this operation. The triangular waveform was tested to determine whether the detailed shape of the ripple and the relationship of RMS to peak changed the measured values. For cases where the comparison of two different current waveforms is based on their having equal amplitudes rather than equal RMS values, these data suggest that the detailed shape does not significantly effect the measured $I_{c}$. The a.c. amplitude is the relevant quantity for scaling the effect on the measured d.c. $I_{c}$.

The data shown in Figures 4 and 5 were taken using the analog nanovoltmeter while the data shown in Figures 6 and 7 were taken using the digital meter. The $60 \mathrm{~Hz}$ data of Figures 6 and 7 were measured on the identical pair of voltage taps as the $60 \mathrm{~Hz}$ data of Figures 4 and

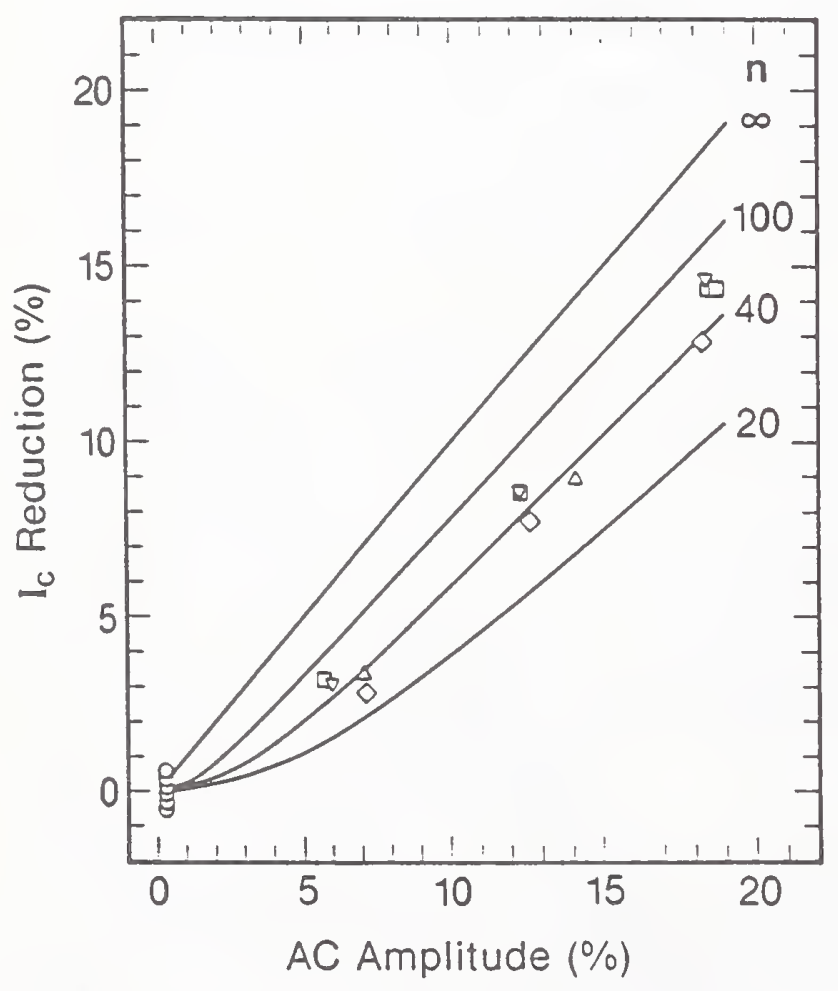

Figure 4 Percentage $I_{c}$ reduction versus the percentage a.c. amplitude. Model curves are shown for several values of $n$. Experimental data taken with an analog voltmeter are shown at several frequencies. The $-60 \mathrm{~Hz}$ frequency was labelled negative to indicate that it was a triangular waveform at $60 \mathrm{~Hz}$ rather than a sinusoidal waveform. Frequency $(\mathrm{Hz}): 0.0 ;[.60 ; \triangle,-60 ; \nabla$ 360: $\uparrow .1000$

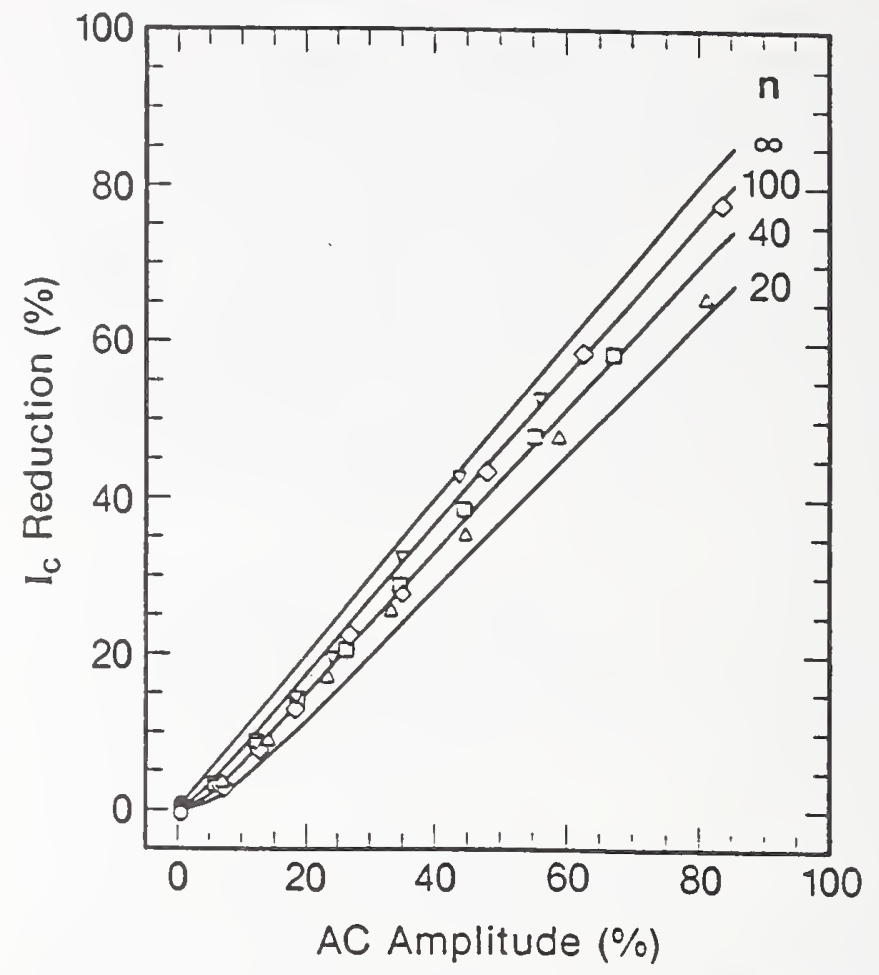

Figure 5 Percentage $/ c$ reduction versus the percentage a.c. amplitude. Model curves are shown for several values of $n$. Experimental d.jta taken with an analog voltmeter are shown at several frequencies. For key to symbols, see Figure 4 caption

5. The digital meter data, at frequencies other than $60 \mathrm{~Hz}$, were measured simultaneously with the corresponding analog meter data but with another pair of voltage taps that spanned the same segment of the sample. This other pair of voltage taps had a higher mutual inductance but the results for both pairs of taps were within the experimental limits. The results measured by the two voltmeters were very close. This indicates that the measured d.c.

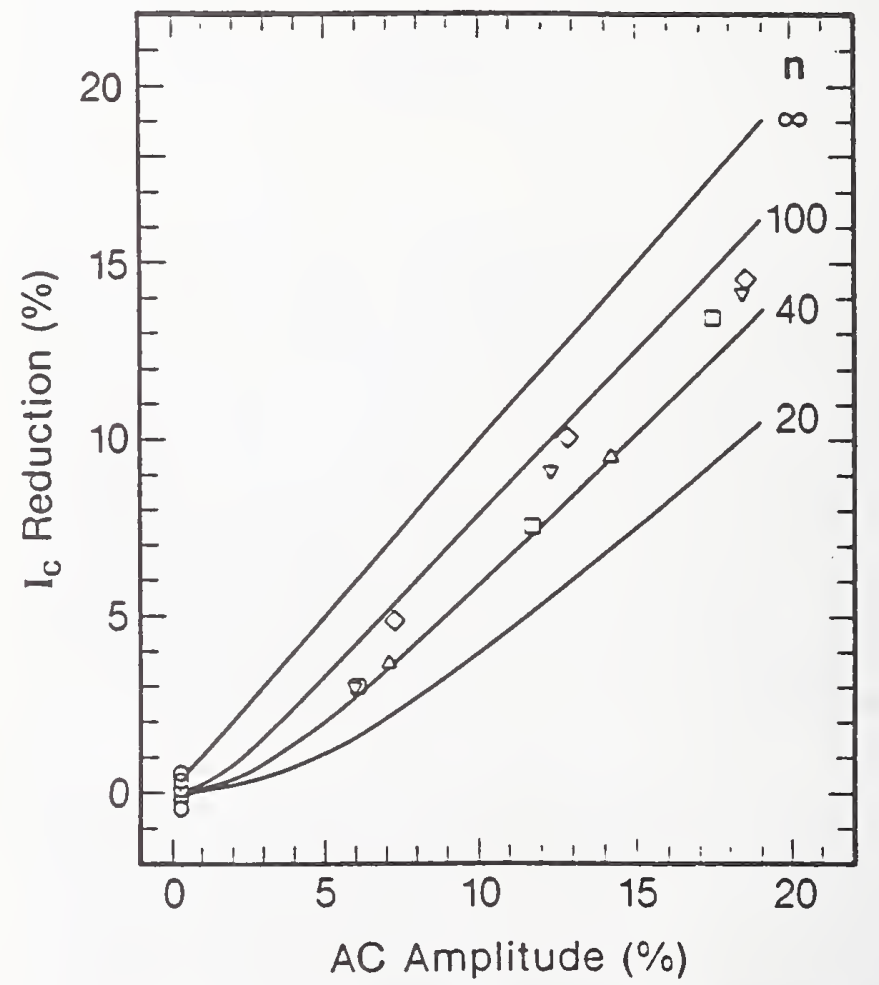

Figure 6 Percentage $I_{c}$ reduction versus the percentage a.c. amplitude. Model curves are shown for several values of $n$. Experimenta! data taken with a digital voltmeter are shown at several frequencies. For key to symbols, see Figure 4 caption 


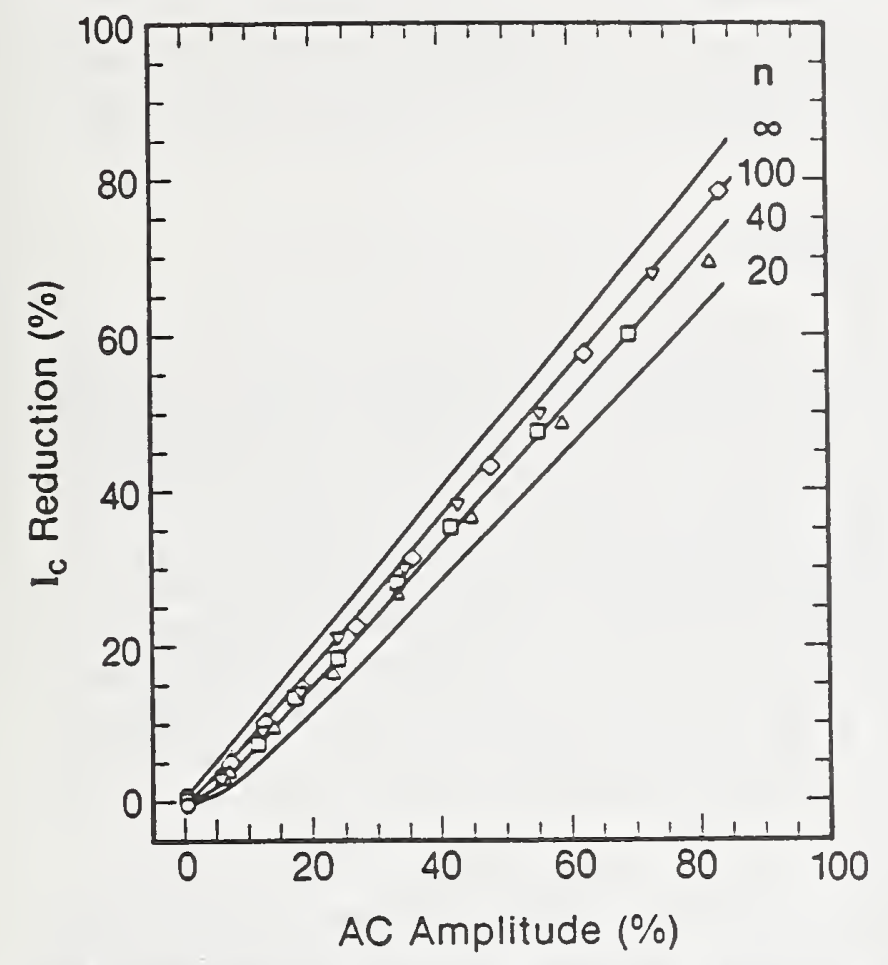

Figure 7 Percentage $/ c$ reduction versus the percentage a.c. amplitude. Model cunes are shown for several values of $n$. Experimental data taken with a digital voltmeter are shown at several frequencies. For key to symbols, see Figure 4 caption

value of $I_{c}$ does not depend on the voltmeter used. There were, however, other differences between these two voltmeters that will be discussed later. The effect of current ripple was measured for a number of sample segments and each segment gave similar results.

The experimental data fall between the $n=20$ and the $n=100$ model curves, within experimental error. The measured value of $n$ was about 30 . A model curve with $n=30$ would be a lower limit to the effect. These experimental values indicate that the effective $n$ may be higher because of the range of electric fields that occur during the peak current ( $n$ is a weak function of $E$ ) or because sample heating increases the value of $n$. The model and data are in close agreement, within $\pm 2 \%$ for ripple below $20 \%$ and within $\pm 10 \%$ for ripple up to $80 \%$ using $n=30$.

The model curves can be used to estimate the effect of current ripple on the measured d.c. critical current. For approximating the effect of commercial power-supply ripple on critical current measurements, the curves of Figures 4 and 6 may be the most useful because of their lower range. Both ripple and the reduction in critical current can be generalized to a percentage effect. Heating through a.c. losses may increase the size of this effect at higher amplitudes depending upon the sample geometry. Voltmeter effects will also become more significant at higher amplitudes.

\section{Discussion}

\section{Artificial current transfer voltage}

The analysed data from both the digital and the analog voltmeters gave the same values of $I_{c}$. However, some $I_{c}$ data taken with the analog nanovoltmeter indicated the presence of a current transfer voltage'. This was not true of the data with the digital meter. It is believed that this behaviour is simply an artifact of the measurement system and not the result of a true current transfer voltage. Two characteristics of the measurement system may explain this effect. One concerns the sample current supply and the other concerns the nanovoltmeter. First, at high a.c. amplitudes the sample current supply's a.c. amplitude increases with increasing d.c. bias level which results in an increasing inductive voltage at the sample voltage taps. Second, as was determined in the symmetric voltage test, the nanovoltmeters have a small d.c. voltage output when subjected to an a.c. input, and this d.c. voltage increases with increasing a.c. amplitude. Consequently, during a cycle of data acquisition, the nanovoltmeter has an increasing d.c. voltage output which is interpreted by the data analysis program as current transfer voltage. The digital meter does not indicate this artificial current transfer voltage because of its considerably greater rejection of symmetric input signals. Finally, good agreement between the data taken by the two meters is achieved by simply subtracting the artificial voltage from the analog meter data. When this current transfer correction is large, significant errors in the determination of the $I_{c}$ can result. This is probably the reason that the high amplitude analog voltmeter data at $360 \mathrm{~Hz}$ (worst case ACR) was out of line with the other frequency data.

\section{A.c. voltage threshold}

Although both analog and digital nanovoltmeters have demonstrated predictable input-output behaviour with respect to low amplitude a.c. input signals, there is an apparent a.c. amplitude threshold above which their behaviour becomes erratic. This erratic behaviour varied from a random output to a very low frequency (less than $1 \mathrm{~Hz}$ ) and high amplitude output. These outputs could be positive or negative. Both meters display this erratic behaviour but the a.c. amplitude threshold for the digital meter is approximately $10 \mathrm{mV}$, whereas the threshold for the analog meter is approximately $1 \mathrm{mV}$. These results are similar to the symmetric test where the digital meter was show'n to have a considerably greater tolerance for a.c. input signals.

\section{Null taps and passive filtering}

Data taken on the null tap pairs (described above) were used to determine the voltmeter's response to the symmetric voltage signals during the experiment. These data were consistent with the symmetric test, the artificial current transfer voltage, and the erratic behaviour, all of which were presented above. This is further evidence for the assertion that these effects are caused by the a.c. inductive voltage. A passive RC filter was inserted at the input of the nanovoltmeter to reduce the a.c. amplitude of the voltage signal. This did reduce the artificial current transfer voltage, and it increased the threshold of the erratic behaviour. However, the passive filter introduced more voltage noise. A passive filter may be an option for some experiments. Another possible method of reducing the inductive voltage is to balance the inductance by opening an opposite area within the twisted pair. This 
area could be adjusted with a high frequency signal during sample preparation.

\section{Transient losses}

The self-field loss is the primary type of transient loss present in the case of sample current ripple for this sample geometry ${ }^{4}$. The only effect of transient loss on the $I_{c}$ measurement is sample heating, which may raise the temperature of the sample depending upon cooling conditions. The power dissipation is a strong function of frequency, amplitude, and current density. Computed self-field loss (for the $30 \mathrm{~cm}$ sample) at $8 \mathrm{~T}$ and the highest a.c. current amplitude was $3 \mathrm{~mW}$ at $60 \mathrm{~Hz}(50 \mathrm{~mW}$ at $1000 \mathrm{~Hz}$ ). The effect of current ripple was also measured at $5 \mathrm{~T}$ with a.c. amplitudes up to about $170 \mathrm{~A}$. The difference in the power between 5 and $8 \mathrm{~T}$ was a factor of 6 for comparable amplitudes and frequencies. There was a slight frequency and amplitude dependence for the $5 \mathrm{~T}$ data (higher frequency or amplitude reduced $I_{c}$ by more), but the results were still at or below the reference line, reduction less than or equal to the a.c. amplitude. The lack of a strong frequency, amplitude, and current density dependence of the measured reduction of $I_{c}$ indicates that sample heating due to transient losses was not significant for this experiment.

Other sample geometries might have larger transient losses due to current ripple. A change in the sample geometry was made to examine this possibility. The sample was centred in a holder, and four conductors (symmetrically located on a $2 \mathrm{~cm}$ diameter circle) were all used for the return current path. This arrangement reduced the effect of the magnetic field from the return current path. $I_{c}$ data was taken at $5 \mathrm{~T}$ with four returns and with one return for various frequencies and a.c. amplitudes. The results with a single return had a slight frequency and amplitude dependence. At $60 \mathrm{~Hz}$ (sinusoidal and triangular), these data were within $1 \%(2 \mathrm{~A})$ of the four-return results. At $360 \mathrm{~Hz}$ and $100 \mathrm{~A}$ a.c. amplitude, the $I_{c}$ was reduced by $3 \%(8 \mathrm{~A})$ as compared to the four-return results. This further reduction is attributed to the additional sample heating due to the transient loss created by the magnetic field of the return current path. For other geometries, such as a coiled sample, this effect could be much larger.

\section{High frequency data}

Additional $I_{c}$ data were taken at higher frequencies ( 3 and $10 \mathrm{kHz}$ ), but experimental difficulties made the results at these frequencies less reliable. Transient losses, inductive voltage, waveform distortion, and reduction in available amplitude all became more significant at higher frequencies. The motivation for investigating higher frequencies was to estimate the effect of frequencies near the range of spikes from silicon-controlled rectifiers (SCR) found in most large power supplies. These data indicated the same trend of reduction in $I_{\mathrm{c}}$ but with more experimental uncertainty. Perhaps a better model of an SCR spike would be a spike rather than a continuous dutycycle waveform. An extrapolation of the work presented here would indicate that the effect of the spikes would only be to add a finite amount to the time-average of the resistive voltage.

\section{Conclusions}

The effect of ripple scales with the percentage of $I_{c}$, and the effect depends upon the shape of the $V-I$ curve (n). For an $n$ of 30 or more and large ripple, current ripple reduces the measured d.c. critical current by slightly less than the a.c. amplitude. The effect of ripple is nearly independent of frequency for the range $60-1000 \mathrm{~Hz}$. A d.c. voltmeter measures a relevant quantity, the time average of the voltage. A simple electronic circuit was used to test a d.c. voltmeter's response to an asymmetric waveform that simulates a superconductor's $V-I$ charac. teristic. Reducing the mutual inductance of the voltage taps is important when testing with power supply ripple present.

A large conductor test method has to address the reality of power supply current ripple. Power line frequencies and harmonics (up to three phase, fully rectified) are the dominant frequencies. Current ripple of $1-3 \%$ at the maximum current is typical for power supplies in the range $600-6000 \mathrm{~A}$. This ripple is generally a function of output current, but the percentage ripple can be higher for intermediate currents. This can be a significant source of measurement error. The direct effect on the sample voltmeter can also introduce measurement error. Sample heating due to transient losses may also be significant for some sample geometries.

\section{Acknowledgements}

The authors extend their thanks to W. E. Look and H. E. Spilman for their assistance with sample prepara. tion and computer programing, and to R. B. Goldfarb for discussions on transient losses. Research supported by the US Department of Energy through the Office of Fusion Energy and the Division of High Energy Physics.

An effort was made to avoid the identification of commercial products by the manufacturer's name or label, but in some cases these products might be indirectly identified by their properties. In no instance does this identification imply endorsement by the National Bureau of Standards, nor does it imply that the particular products are necessarily the best available for that purpose.

\section{References}

1 Goodrich, L.F. and Fickert, F.R. Critical current mcasurements: a compendium of experimental resulis Cryog(nics (1982) 22225

2 Bray, S.L and Guodrich, L.F. Battery powered current supply for supcrconductor measurements National Bureau of Siandards N'BSIR $\& 8-3088$ (1988)

3 Goodrich L.F. ef al. Development of standards for superconductors, interim report Jan. to Dec. 1985 National Bureau of Siandards NBSIR 87-3066 (1987)

4 Ekin, J.W. Malcrials at Low Temperature American Society for Mctals, USA (1983) Ch 13 


\section{APPENDIX K}

CALORIMETRIC MEASUREMENTS OF AC LOSS IN $\mathrm{Nb}_{3} \mathrm{Sn}$ SUPERCONDUCTORS

M. Takayasu, C. Y. Gung, M. M. Steeves, B. Oliver, D. Reisner, and M. O. Hoenig*

published in:

Eleventh International Conference on Magnetic Technology (MT-11) Tsukuba, Japan, 1989

* None of these authors are NIST employees. 
Eleventh International Conference on Magnet Technology (MT-11)

Tsukuba, Japan, 1989

\title{
CALORIMETRIC MEASUREMENT OF AC LOSS IN Nb3 Sn SUPERCONDUCTORS *
}

\author{
M. Takayasu, C. Y. Gung, M. M. Steeves, B. Oliver, D. Reisner and M. O. Hoenig \\ Massachusetts Institute of Technology \\ Plasma Fusion Center \\ Cambridge, Massachusetts 02139, USA
}

\begin{abstract}
The A.C. loss of twisted internal-tin, modified-jelly-roll $\mathrm{Nb}_{3} \mathrm{Sn}$ wire intended for the US-DPC coil was measured in triangular wave fields at ramp rates up to $1.3 \mathrm{~T} / \mathrm{s}$ by an isothermal calorimetric method. To minimize the time delay of measurement, a constant-flow control method was developed. Measurement with ripple and triangular fields allowed hysteresis loss and coupling loss to be separated. Hysteresis loss obtained by the present calorimetric apparatus agreed quite well with magnetization measurements obtained at NIST. Conclusions drawn from the preliminary tests indicate the major component of A.C. loss to be hysteresis below $2 \mathrm{~T} / \mathrm{s}$ for the observed low values of the residual resistivity ratio, RRR $(10-30)$. The bundle coupling loss will become comparable or even dominant at the expected maximum operational ramp rate of $9.5 \mathrm{~T} / \mathrm{s}$, especiaily if the RRR values were to improve significantly. Estimates of A.C. loss for the US-DPC coil operating at JAERI are made.
\end{abstract}

\section{INTRODUCTION}

The United States Demonstration Poloidal Coil (US-DPC) is being built in order to test advanced, high-current-density, pulsed $\mathrm{Nb}_{3} \mathrm{Sn}$ cable-in-conduit superconductors for ohmic heating coils for tokamak fusion devices [1]. AC loss is one of the critical factors for the US-DPC Coil, which uses commercial $\mathrm{Nb}_{3} \mathrm{Sn}$ wire. The superconducting wire was manufactured by Teledyne Wah Chang Albany (TWCA). It is titanium-alloyed internal-tin, jelly-roll $\mathrm{Nb}_{3} \mathrm{Sn}$ wire of the diameter $0.78 \mathrm{~mm}$ with an internal $\mathrm{Cu} / \mathrm{Nb}$ ratio of 1.821 with a vanadium barrier, designed to maximize current density while minimizing A.C. loss [2]. The nominal non-copper current density is $700 \mathrm{~A} / \mathrm{mm}^{2}$ at $10 \mathrm{~T}$ [3]. A.C. loss measurements of the 225 strand, $30 \mathrm{kA}$ US-DPC conductor will be carried out at ramp rates of up to $9.5 \mathrm{~T} / \mathrm{s}$ in early 1990 at the Demonstration Poloidal Coil Facility (DPCF) at JAERI, Japan.

In order to perform laboratory measurements of A.C. loss, an isothermal calorimeter was built in which loss is measured as a

* Supported by US DOE Contract DE-AC02-78ET51013. function of helium boil-off. The calorimeter permitted the loss measurement of $12 \mathrm{~m}$ long single strand samples $(5 \mathrm{cc}$ ) in a continuous triangular wave field of a $12 \mathrm{~T}$ Bitter magnet at the Francis Bitter National Magnet Laboratory, MIT. To minimize measurement time delay, a constant flow control method was developed. Tests of single strand samples of TWCA US-DPC wire were performed with triangular wave fields at ramp rates of up to $1.3 \mathrm{~T} / \mathrm{s}$. Ripple field loss test were also performed. Together, the triangular field and ripple field tests allowed hysteresis and coupling loss to be mathematically separated. The coupling loss is mainly bundle coupling loss, that is, an eddy current loss between adjacent filament bundles. Based on these measurements, an estimate of the A.C. loss of US-DPC coil under test conditions is given.

\section{EXPERIMENTAL}

\section{APPARATUS}

A calorimetric apparatus [4] was developed to measure the A.C. loss of single strands, as well as cabled superconductors, with and without transport current. Fig. 1 shows a schematic of the 


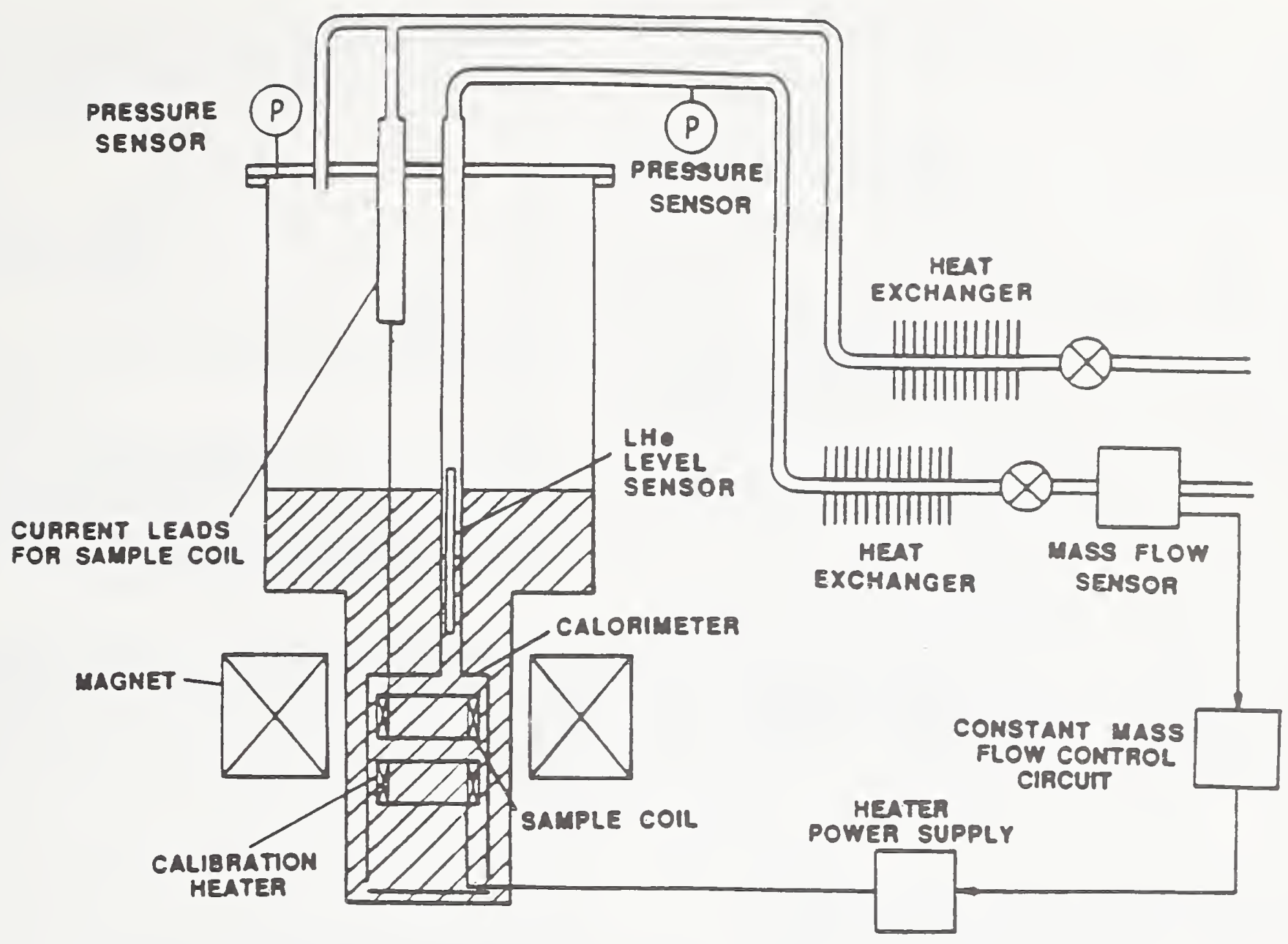

Fig. I Schematic diagram of calorimetric A.C. loss measurement apparatus.

apparatus. A $12 \mathrm{~m}$ long single strand (MIT designation 87-17) was first wound non-inductively in a single layer on a $100 \mathrm{~mm}$ diameter, $75 \mathrm{~mm}$ long machinable ceramic, and was subsequently heat treated. The test sample was enclosed by an open bottom bell jar in the $127 \mathrm{~mm}$ inner diameter tail section of a metal cryostat. The helium boil-off was measured at room temperature by a mass flow meter. The apparatus was calibrated by a D. C. resistive heater.

A constant flow control system was used in order to minimize the time delay required to establish an equilibrium state of the flowing helium gas. Constant mass flow was maintained by an automatic feedback system which controlled a background heater. The sensitivity was about 5 $\mathrm{mW}$, and the resolution was less than $1 \mathrm{~mW}$. Typical A.C. loss measurements ranged from 20 $40 \mathrm{~mW}$.

\section{TEST RESULTS}

$4.2 \mathrm{~K}$,

Measurements of $\mathrm{AC}$ loss were made at

A) by means of high frequency ripple fields at steady DC fields and,

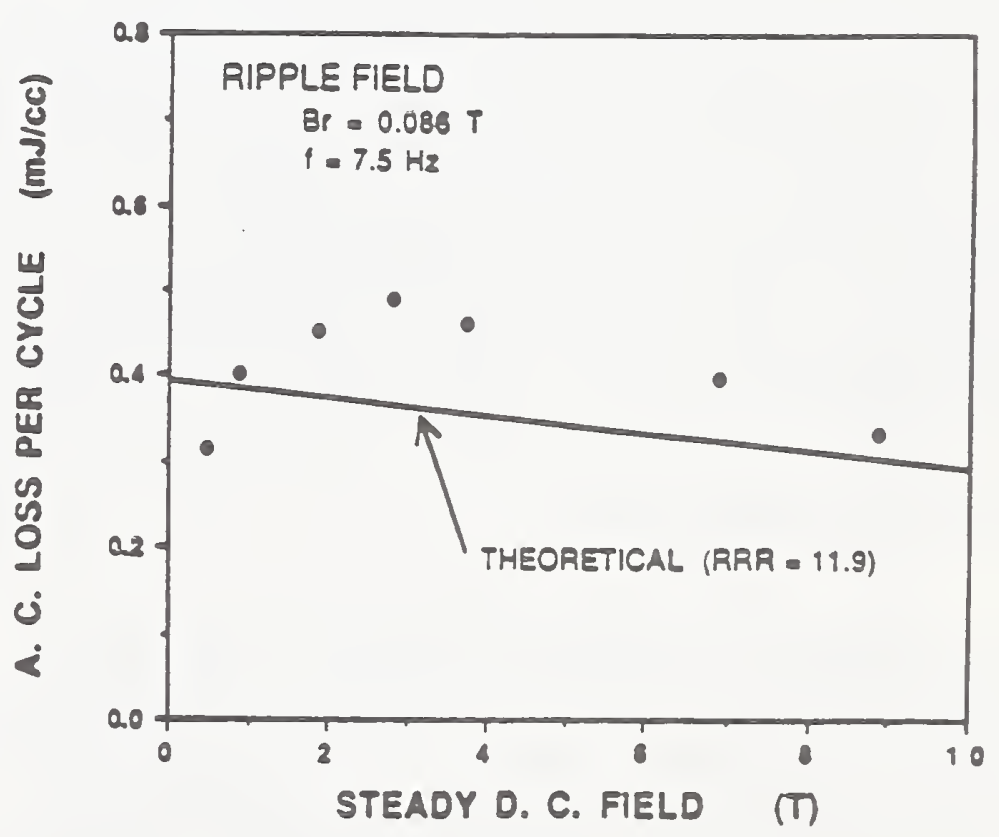

Fig. 2 Experimental data of total A.C. loss at $4.2 \mathrm{~K}$ due to a ripple field as a function of steady D.C. field with the superimposed ripple field of peak-topeak amplitude $B_{r}=0.086 \mathrm{~T}$ and frequency $f=7.5$ $\mathrm{Hz}$. The solid line shows theoretical loss using measured RRR of 11.9 . 


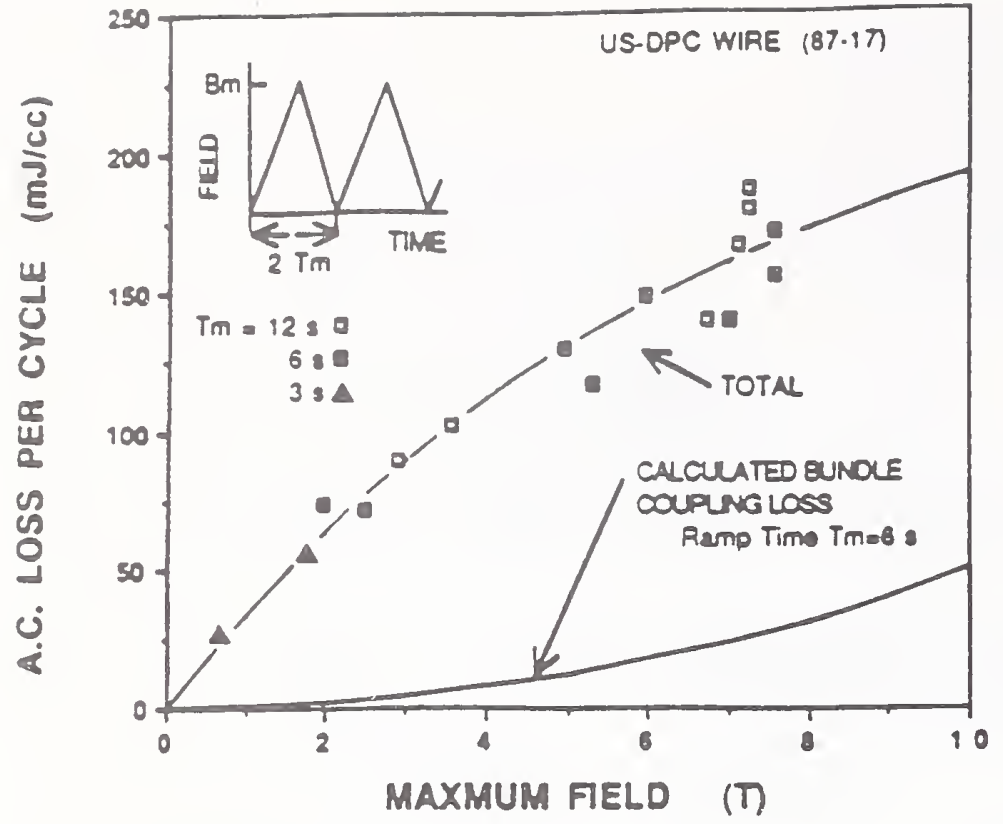

Fig. 3 Measured total A.C. loss at $4.2 \mathrm{~K}$ due to a ramp field as a function of the maximum field $B_{m}$. The lower solid line shows theoretical bundle coupling loss with the ramp time $T_{m}=6 \mathrm{~s}$.

B) by means of triangular wave ramped fields of up to 9 teslas with maximum ramp rates of $1.3 \mathrm{~T} / \mathrm{s}$.

\section{A. RIPPLE FIELD TESTS}

Fig. 2 shows A.C. loss due to a ripple field as a function of D.C. field. The frequency and the amplitude (peak-to-peak) of the ripple field were $0.086 \mathrm{~T}$ and $7.5 \mathrm{~Hz}(1.3 \mathrm{~T} / \mathrm{s})$, respectively. The solid line is calculated from the existing theoretical equation [5], taking RRR to be 11.9 , a measured value of the tested wire. The negative slope of the solid line is due to the magnetic effect on the electrical resistivity of the copper matrix. The agreement between the experimental data and the calculated values is fairly good, except that the experimental points show a peak around $3 \mathrm{~T}$ of the D.C. field.

\section{B. RAMP FIELD TESTS}

Fig. 3 shows measured total loss due to a ramped field as a function of maximum field with various ramp times. The lower solid line calculated using existing theory shows the bundle coupling loss [5]. The difference between the total loss and the calculated bundle coupling loss appears to be hysteresis loss, plotted in Fig.4.

The four solid circles in Fig. 4 show the hysteresis loss measured by magnetization [4]. Original data were full-cycle hysteresis losses. They were reduced to half-cycle loss using

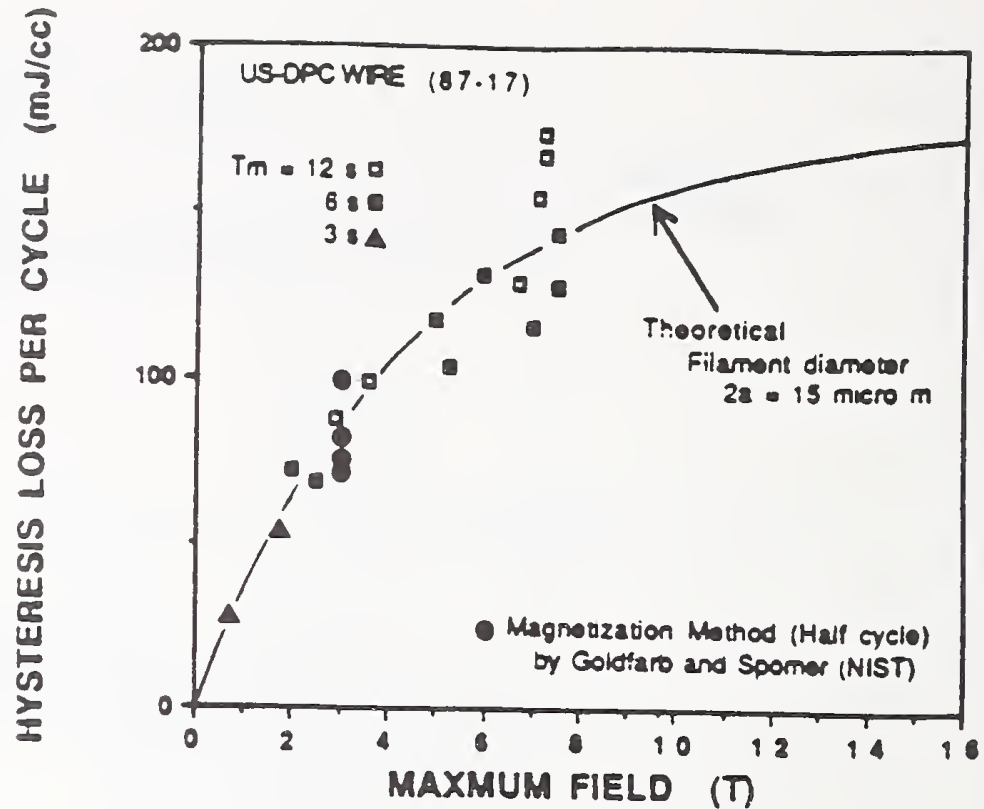

Fig. 4 Hysteresis loss obtained by the subtraction of the calculated bundle coupling loss from the measured total loss shown in Fig. 3. The solid line is calculated from Eqs. (1) and (2) with $a=7.5$ microns.

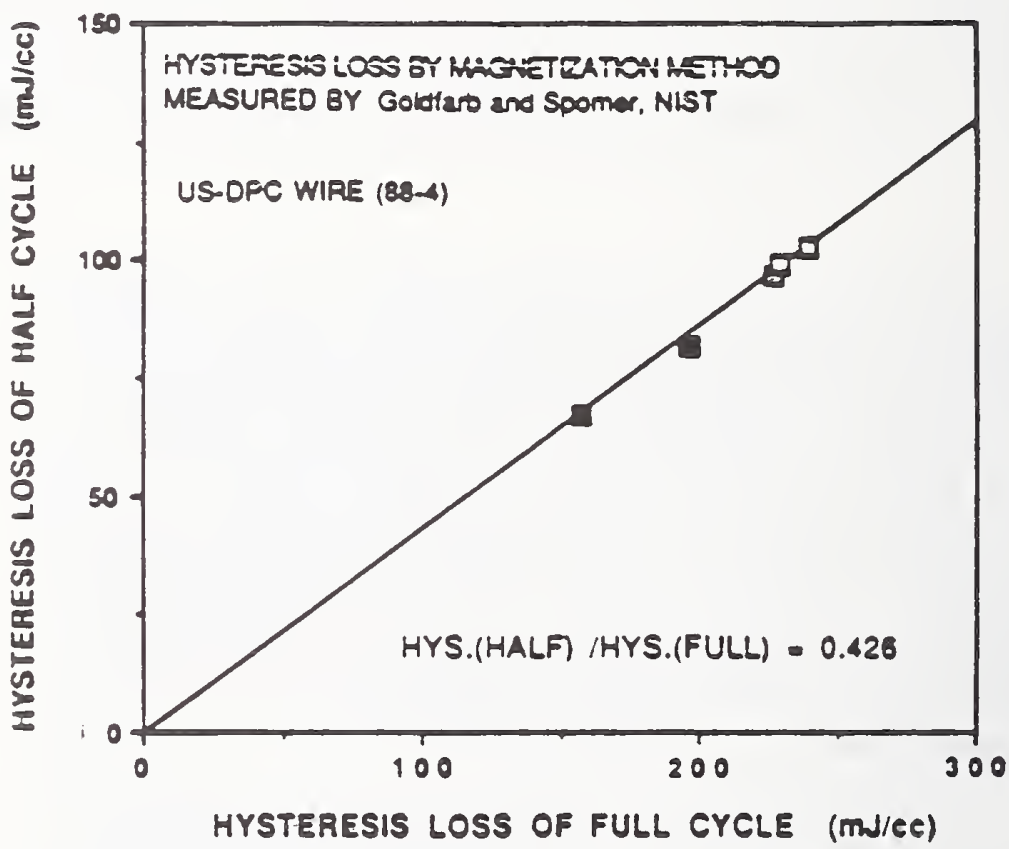

Fig. 5 Relationship between the half cycle hysteresis and full cycle hysteresis losses, which were measured by the magnetization method at NIST. The maximum field values are 3T ( $\square)$ and 7T (ם). The half cycle hysteresis loss is seen to be $42.6 \%$ of the full cycle one.

magnetization data obtained experimentally for similar TWCA US-DPC wire. The comparison is shown in Fig.5. The half cycle hysteresis loss was $42.6 \%$ of full cycle loss. 
If a superconductor has no transport current, the hysteresis loss per unit volume per cycle may be given by Eq. (1) [5].

$$
Q_{h}=\frac{8}{3 \pi} \int_{0}^{B_{m}} J_{c} a d B
$$

where $J_{C}$ is the critical current as a function of the field $B, B_{m}$ is the maximum field and $a$ is the filament radius.

An approximation of the critical current $J_{C}$ with the field $B$ is given by the following equation obtained experimentally by tests using similar USDPC wires at magnetic fields between $8 \mathrm{~T}$ and $15 \mathrm{~T}$.

$$
J_{C}=6.117 \times 10^{3} \exp (-0.219 B)\left[\mathrm{A} / \mathrm{mm}^{2}\right]
$$

The solid line in Fig, 4 is based on Eq. (1) with Eq. (2), taking the filament diameter to be 15 microns. While the filament size is larger than expected, an effective filament size would be larger than the design value ( 3 microns) or optically observed filament size (about 6 microns). Agreement between the experimental data and the calculation is qualitatively significant.

\section{MEASUREMENT ACCURACY}

The accuracy of A. C. loss measurement using our calorimetric technique depends on the following:

(1) Extraneous boil-off caused by current leads, cryostat, and other structure materials.

(2) Heat transfer through the bell jar.

It is caused by temperature difference of the helium boiling point due to the pressure difference between helium inside and outside of the bell jar.
(3) Acoustic resonance in the vent tube. It is high frequency oscillation noise of helium vapor flow.

(4) Leakage of helium gas.

(5) Mass flow change due to the time-varying magnetic field.

One disadvantage of the calorimetric method is that the time required to reach equilibrium (usually takes more than $40 \mathrm{~min}$ ) [4]. By using the constant flow control system, the time required can be reduced to less than $10 \mathrm{~min}$. In this way, the operating pressure and helium bath temperatures can be kept constant during measurement.

When pressure outside the bell jar in the cryostat changes, undesirable heat transfer occurs through the vent tube wall. In order to prevent this, the vent tube was originally vacuum shielded. The high frequency acoustic noise (problem (3), above) was however enhanced. The noise became negligible when a single layer vent tube was used without a vacuum shield. By adopting the constant flow operation, the pressure fluctuations in the cryostat during measurement could be minimized.

Leakage of boil-off vapor from calorimeter in liquid helium was increased with both D.C. and A.C. magnetic fields (problem (4), above). This problem was solved using Stycast 2850FT to seal the $\mathrm{G} 10$ canister.

Problem (5), not yet fully understood, causes the most serious background noise for an accurate loss measurement. When a triangular waveform is applied, the mass flow change per unit time due to this noise depends on the amplitude of magnetic field, but not ramp time. This means that the noise effect on the measurement loss per cycle proportionally increases with ramp time. The data obtained with more than $30 \mathrm{sec}$ of the ramp time had a large error due to this noise. The data shown in Figs. 3 and 4 were obtained with a ramp time $T_{m}$ of $12 \mathrm{sec}$ or less. The resultant error was less than $10 \%$.

Table 1. Measured and design values of the half cycle hysteresis loss per cycle in unit of $\mathrm{mJ} / \mathrm{cc}$.

\begin{tabular}{ccc}
\hline $\begin{array}{c}\text { Maximum Field } \\
\mathrm{B}_{\mathrm{m}}(\mathrm{T})\end{array}$ & $\begin{array}{c}\text { Present Calorimetric } \\
\text { Method }\end{array}$ & $\begin{array}{c}\text { Magnetization } \\
\text { Method (NIST) }\end{array}$ \\
\hline 3 & 86 & $83^{*}$ \\
7 & 140 & \\
\hline \multirow{2}{*}{$* 42.6 \%$ of the full cycle hysteresis loss measured. }
\end{tabular}




\section{DISCUSSION AND CONCLUSIONS}

The A.C. loss test results obtained by the calorimetric method have an error of less than $10 \%$. The hysteresis loss per cycle of US-DPC wires with a triangular wave, Eq. (3), was obtained using Eqs.(1) and (2) with a computer fitting to the experimental data shown in Fig. 4.

$$
\mathrm{Q}_{\mathrm{b}}=178.2\left\{1 \cdot \exp \left(-0.219 \mathrm{~B}_{\mathrm{m}}\right)\right\}[\mathrm{mJ} / \mathrm{cc}]
$$

Table 1 shows both the half cycle hysteresis losses obtained from Eq. (3) and using the magnetization method. As discussed above, the values obtained by the magnetization method are reduced to the half cycle hysteresis loss by taking $42.6 \%$ of the full cycle values. The half cycle hysteresis loss per cycle of US-DPC wire was 86 $\mathrm{mJ} / \mathrm{cc}$ for a 0 - 3T - 0 cycle and $140 \mathrm{~mJ} / \mathrm{cc}$ for a 0 $7 \mathrm{~T}-0$ cycle. These values agreed quite well with values obtained by the magnetization method at NIST.

The other major A.C. loss component is the bundle coupling loss. As shown in Fig. 3, however, bundle coupling loss was minor in our experimental range of frequency. The coupling loss increases with the ramp rate and copper matrix resistivity. The effect of magnetic field on copper resistivity or RRR must be taken into account. Even if RRR is as large as 1000 without magnetic field, the value is decreased to less than 40 at a magnetic field of 10T. If the copper resistivity is a linear function of magnetic field $B$, given as $\rho(B, 4.2 K)=\rho_{0}(B=0,4.2 K)+\alpha B$ [Ohm m], the coupling loss per cycle due to the triangular wave can be given using existing theory [5] by

$$
Q_{c}=\left(\frac{l_{p}}{2 \pi}\right)^{2} \frac{2 B_{m}}{\alpha T_{m}} \ln \left(\frac{\rho_{0}+\alpha B_{m}}{P_{0}}\right)
$$

In Eq. (4), $l_{p}$ is the twist pitch and $\rho$ is the effective transverse resistivity. Since $\mathrm{l}_{\mathrm{p}}$ of the wire is 9.8 $\mathrm{mm}$, and if $p_{0}=2.0 \times 10^{-8} / \mathrm{RRR}$ [Ohm m] and $\alpha=4.8$ $x 0^{-11}[\mathrm{Ohm} \mathrm{m} / \mathrm{T}]$, the coupling loss per cycle is given as follows,

$$
\mathrm{Q}_{\mathrm{c}}=50.68 \frac{\mathrm{B}_{\mathrm{m}}}{\mathrm{T}_{\mathrm{m}}} \ln \left(1+2.4 \times 10^{-3} \mathrm{RRR} \mathrm{B}_{\mathrm{m}}\right)[\mathrm{mJ} / \mathrm{cc}]
$$

Fig. 6 shows the bundle coupling loss calculated using Eq. (5), as a function of the ramp rate of a triangular wave field. The maximum field value $B_{m}$ is 10T. The hysteresis loss per cycle is also shown in the figure. It is independent of ramp

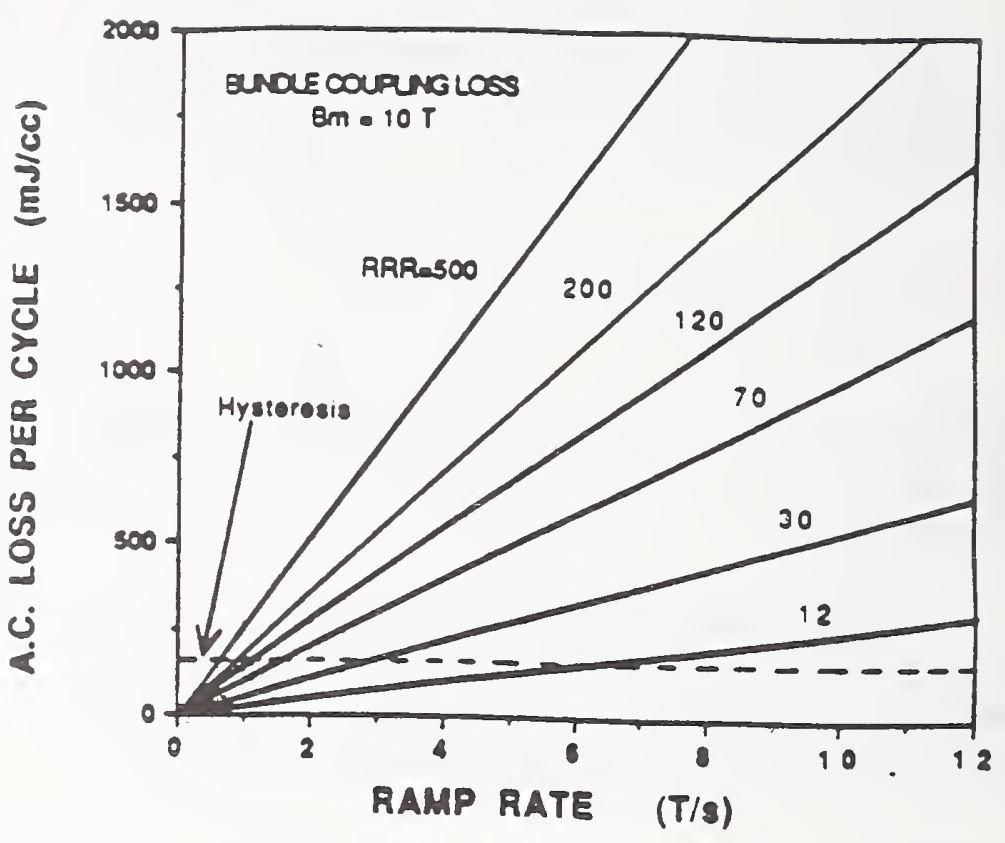

Fig. 6 The expected bundle coupling loss of US$D P C$ wire for various $R R R$ values as a function of the ramp rate of a triangular wave field at the maximum field of $B_{m}=10 T$. The hysteresis loss is also shown by a dotted line.

rate. As seen in Fig. 6, the bundle coupling loss will be a few times larger than the hysteresis loss at the expected maximum US-DPC operating ramp rate of $9.5 \mathrm{~T} / \mathrm{s}$.

Conclusions drawn so far from the tests with triangular and ripple waveforms indicate the major component of A.C. loss for a twisted TWCA US-DPC wire to be hysteresis below $2 \mathrm{~T} / \mathrm{s}$ for the observed low values of the residual resistivity ratio, RRR (10 - 30). However, the bundle coupling loss (eddy current loss between adjacent filament bundles) will become comparable or even dominant at the expected maximum operating ramp rate of 9.5 $T / s$, especially if the $R R R$ value were to improve significantly. It is noted that the RRR value of A.C. superconductor must be optimized to provide maximum protection at minimum A.C. loss.

The US-DPC is composed of three double pancakes. The length of each pancake is $75 \mathrm{~m}$. The coil will be operated in series with DPC-U1 and U2 of JAERI using a continuous triangular wave current, ramped between 0 and $30 \mathrm{kA}$ (maximum) in a few seconds. The maximum field, which the innermost turn will be exposed to, is 10T. By using the calculated field distribution of the USDPC conductor, both the hysteresis loss and the bundle coupling loss were calculated from Eqs. (3) and (5), respectively. Fig. 7 shows the results obtained with $R R R=30$ under the most severe conditions (maximum current of $30 \mathrm{kA}$, and ramp 


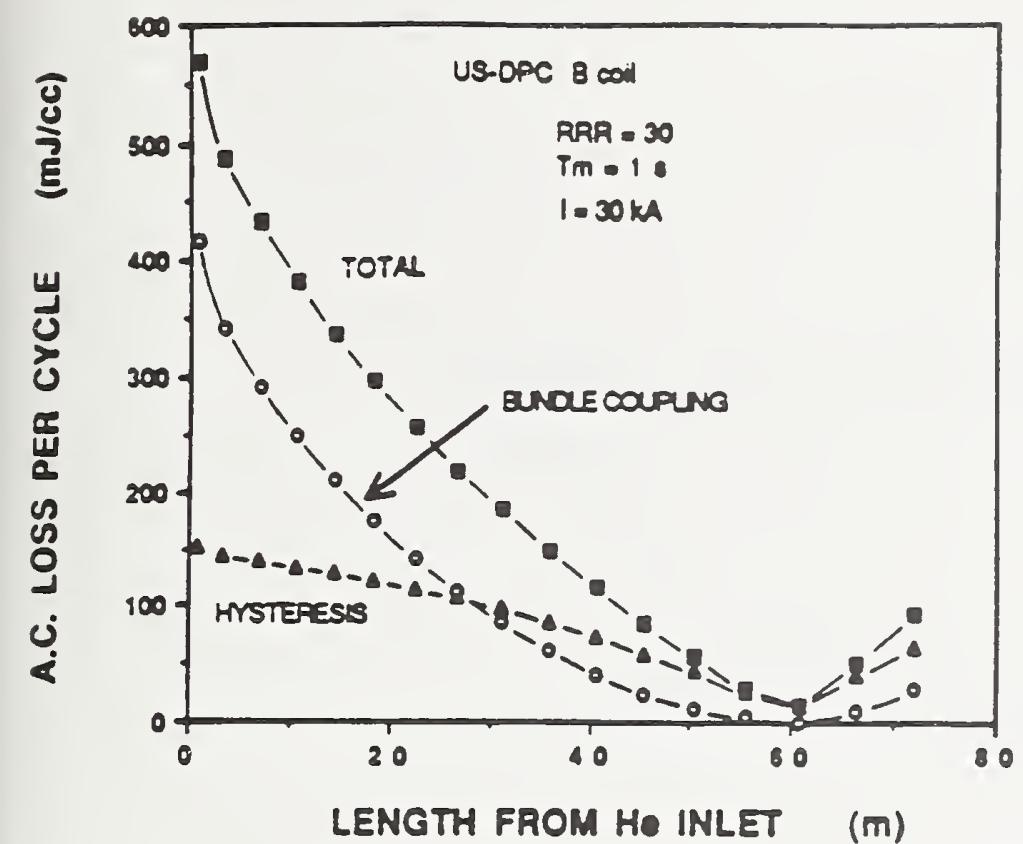

Fig. 7 Calculated A.C. loss per cycle per unit volume along the conductor of the center pancake of US-DPC.

time $T_{m}$ of 1s). Here the A.C. loss per cycle is plotted as a function of position along the conductor of the center pancake from the point of helium entry. As seen in this figure the bundle coupling loss is dominant at the inner turns.

The A.C. loss power, that is loss per unit time, can be given by dividing Eqs. (3) and (5) by the cycle time $2 \mathrm{~T}_{\mathrm{m}}$. Calculated total A.C. loss power per second of the whole US-DPC coil with the maximum current of $30 \mathrm{kA}$ and RRR of 30 is shown in Fig.8 as a function of ramp time. The coupling loss becomes comparable or dominant with $\mathrm{T}_{\mathrm{m}}$ of $1 \mathrm{~s}$ (the expected maximum operating ramp rate). The maximum total A.C. loss expected under these operating conditions is about $5 \mathrm{~kW}$.

\section{ACKNOWLEDGMENT}

The authors wish to thank T. Ichihara on leave from Mitsubishi Electric Corp. and S. H. Autler of University of Massachusetts for their initiation of the calorimeter and helpful discussions, and are also grateful to Y. Iwasa for helpful

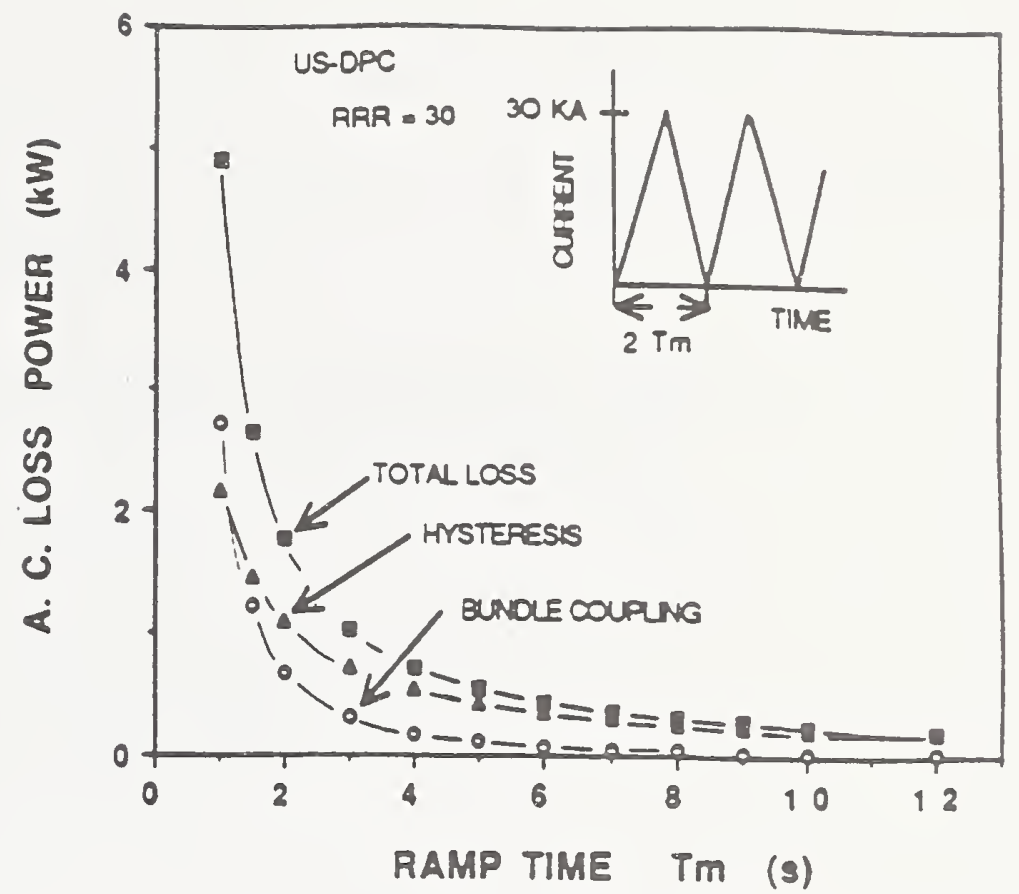

Fig. 8 Calculated A.C. loss power per second of the whole three double pancakes of US-DPC.

suggestions, and J. S. Schultz for magnetic field analysis of US-DPC coil. The authors are indebted to R. B. Goldfarb and R. C. Spomer of NIST for hysteresis measurements by magnetization method.

\section{REFERENCES}

1. Steeves, M.M. et al, The US-DPC, a poloidal coil test insert for the Japanese demonstration poloidal coil test facility. IEEE Trans. Mag., 1988, 24, 1307-1310.

2. Hoenig, M.O., Steeves, M.M. and Gibson, C.R., The selection of 30kA ohmic heating coil conductor. IEEE Trans. Mag., 1988, 24, 1452 1454.

3. Smathers, D.B. et al, Production of tin core modified jelly roll cable for the M.I.T. multipurpose coil. IEEE Trans. Mag., 1988, 24, 1131-1133.

4. Goldfarb, R.B., Ekin, J.W., Hysteresis losses in fine filament internal-tin superconductors. Cryogenics, 1986, 26, 478-481.

5. For example, Wilson, M.N., Superconducting, Clarendon press,Oxford,1983, pp.162-182. 



\title{
APPENDIX L
}

GUIDELINE FOR $\mathrm{Nb}_{3} \mathrm{Sn}$ CRITICAL CURRENT MEASUREMENTS USING FIBERGLASS-EPOXY COMPOSITE SAMPLE MANDRELS (SUMMARY)

\author{
L. F. Goodrich \\ Presented at VAMAS TWP meeting \\ International Cryogenic Materials Conference \\ Los Angeles, California \\ 23 July 1989
}




\title{
Guideline for $\mathrm{Nb}_{3} \mathrm{Sn}$ Critical Current Measurements Using Fiberglass-Epoxy Composite Sample Mandrels (Summary) \\ L. F. Goodrich
}

\author{
Superconductor and Magnetic Measurements Group \\ National Institute of Standards and Technology \\ Boulder, Colorado \\ Presented at VAMAS TWP meeting \\ International Cryogenic Materials Conference \\ Los Angeles, California, 23 July 1989
}

The materials used for this technique are stainless steel tubes for reaction mandrels, G-10CR cylinders cut from plate stock (plate tubes, see Fig.1) for measurement mandrels, and silicone-based vacuum grease as the bonding material.

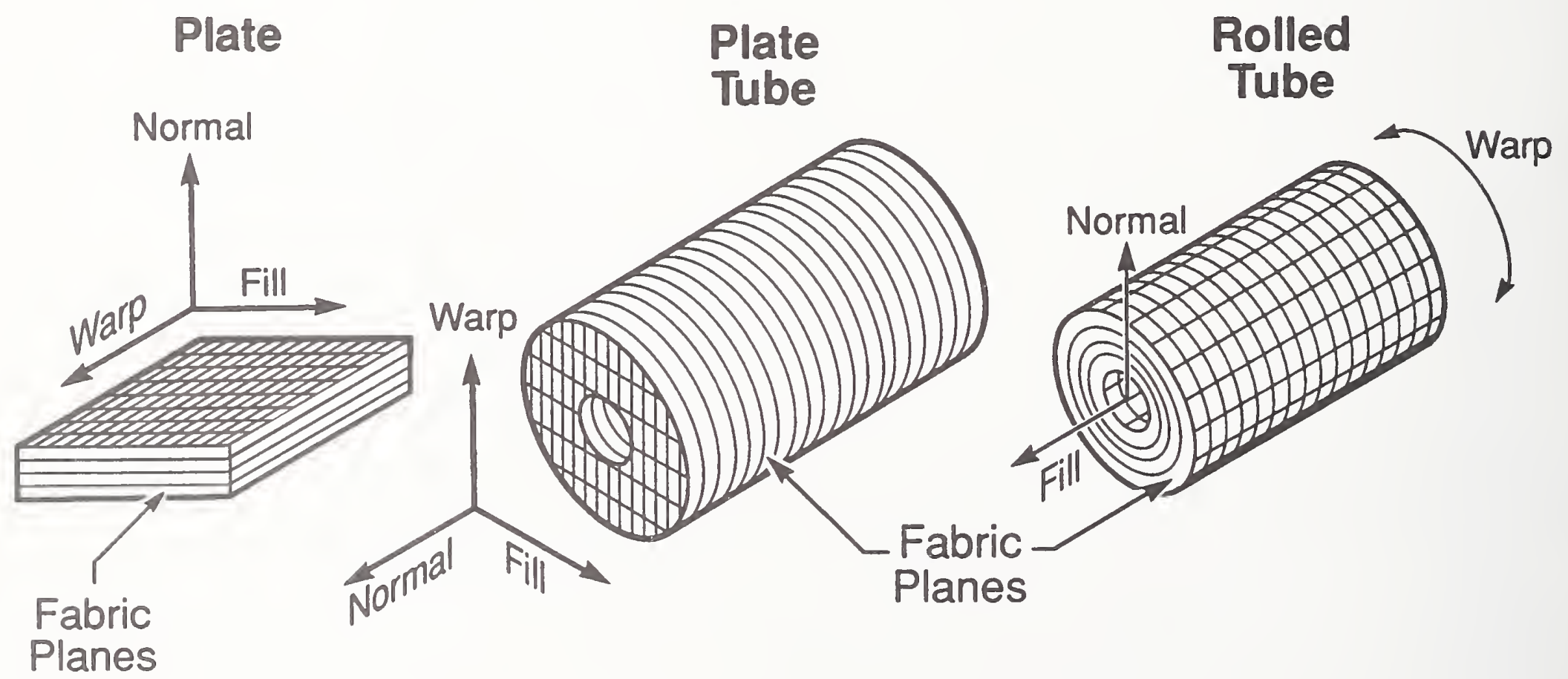

Fig. 1. Illustration of fiberglass-epoxy geometries. 


\section{Preface}

This guideline and others were requested as an attempt to achieve consistent interlaboratory measurements.

A competition exists between the benefit of a rigidly specified measurement technique, which ensures measurement reproducibility, and a less restrictive technique that would be practical for a greater number of laboratories.

This measurement guideline is intended to be one of a few different measurement techniques in a second $\mathrm{Nb}_{3} \mathrm{Sn} \mathrm{I}_{\mathrm{C}}$ round robin that is envisioned to determine the preferred measurement method for $\mathrm{Nb}_{3} \mathrm{Sn}$.

At this time, there is no consensus regarding the materials used for the reaction mandrel, measurement mandrel, or sample bonding.

There are advantages and disadvantages for all known materials.

This guideline was written for one combination of materials.

It may not be the best, but it represents one feasible option. 


\section{Sample Reaction Mandrel}

Helical grooved (not too deep) and oxidized tube.

Well seated, but not tight sample.

Bend wire through holes on each end to retain coil.

Internal-tin processed wire ends outside furnace.

\section{Sample Measurement Mandrel}

G-10 "plate tube" (see Fig. 1).

Same pitch length and groove geometry as reaction.

Dia. approximately $0.5 \%$ larger than reaction mandrel.

Thin layer of grease in groove.

Sample well seated in groove.

\section{Sample Mounting}

Check for diffusion bonding.

If bonded, the sample should be discarded.

Two methods of removing sample from reaction mandrel, unthreading or slightly expanding coil.

If threading onto measurement mandrel, then apply grease to groove with sample up between grooves. 


\section{Sample Mounting (cont.)}

Solder one current contact, then remove the coil's slack, hold the sample taut and solder the other current contact.

Neither contact should have more than 0.9 turns otherwise a slowly decaying current can be induced.

Attach voltage taps to wire after it is seated.

\section{Sample Cooling Rate}

Grease has no mechanical strength until it freezes

May result in different mechanical states for samples cooled at different rates.

Cool sample over a time period of at least 5 minutes.

\section{Current Reversal}

Grease has a relatively low tensile strength.

Lorentz force on the sample coil directed inward only.

\section{Field History and Repeat Determinations}

First measurement at a magnetic field closest to where the Lorentz force is maximum.

Repeat determination of $\mathrm{I}_{C}$ at this first magnetic field. 


\section{Discussion}

Diffusion bonding is a significant problem.

Baking in a sprayed graphite lubricant may help.

Thermal expansion of reaction mandrel may be a problem because the wire may shorten upon anneal.

Does this lead to alumina or a stainless steel with an alumina coat?

A low wall-to-radius ratio $\mathrm{G}-10$ or $\mathrm{G}-11$ rolled tube may be another option for a measurement mandrel that is well matched in thermal contraction to a $\mathrm{Nb}_{3} \mathrm{Sn}$ conductor.

Grease may not be necessary and it may have an adverse effect.

The angle of the groove may have an effect; $90^{\circ}$ may be better than $60^{\circ}$.

\section{Cautions}

This was not written as a step-by-step procedure.

This is a short version of this guideline; see the text version for more complete details. 


\section{Standardization of Coil Dimensions}

\section{Serious consequences to the selection of a coil diameter}

If the coil diameter is selected to allow measurements in the smallest magnet bore, then the probability of sample damage due to handling increases.

If a large coil diameter is selected, a number of laboratories may not be able to participate.

\section{Significant benefits to standardizing coil diameter}

This may be the most effective way to achieve consistent $I_{C}$ measurements.

Batch fabrication of reaction and measurement mandrels with common materials could be cost effective and allow more interchangeable combinations of reaction and measurement mandrels.

This could facilitate the exchange of samples among laboratories. 

APPENDIX M

REPORT ON SAMPLE MOUNTING

L. F. Goodrich

Presented at VAMAS TWP meeting International Cryogenic Materials Conference

Los Angeles, California

23 July 1989 


\title{
Report on Sample Mounting
}

\author{
L.F. Goodrich
}

Superconductor and Magnetic Measurements Group

National Institute of Standards and Technology

Boulder, Colorado

Presented at VAMAS TWP meeting

International Cryogenic Materials Conference

Los Angeles, California, 23 July 1989 


\section{Reaction Mandrels}

\section{Requirements}

* Need grooves and retainers.

* Want to avoid diffusion bonding.

* Thermal expansion may be important.

\section{Options}

* Oxidized stainless steel.

- Easy to machine.

- Diffusion bonding can be a problem.

- High thermal expansion.

* Stainless steel with ceramic coat.

- Cost and adherence of ceramic?

- Thermal expansion is still high.

* Alumina

- Cost of machining is high.

- Durability?

- No diffusion bonding.

- Low thermal expansion. 


\section{Measurement Mandrels}

\section{Requirements}

* Reversible sample bonding.

* Thermal expansion is important.

\section{Options}

* G-10 rolled tubes with low wall/radius and grease bonding.

- Easy to machine.

- Thermal contraction can be matched or lower. (tighten coil)

- Thermal contraction may vary.

- Thin layer of grease or bare.

* G-10 plate tubes with grease bonding

- Hard to machine.

- Thermal contraction lower than $\mathrm{Nb}_{3} \mathrm{Sn}$ wire. (tighten coil)

- Thermal contraction not variable.

- Thin layer of grease or bare. 


\section{Measurement Mandrels (cont.)}

* Stainless steel with grease bonding.

- Easy to machine.

- Thermal expansion slightly more than $\mathrm{Nb}_{3} \mathrm{Sn}$ wire?

- Grease may support.

- Augmented sample stability may be a problem.

- Shunting current may not be a problem.

- Could use the same mandrel for measurement and reaction. (differential expansion?)

* Stainless steel with solder bonding.

- Easy to machine.

- Thermal contraction more than $\mathrm{Nb}_{3} \mathrm{Sn}$ wire?

- Augmented sample stability is a problem.

- Shunt Current may be a problem.

- Can not use same mandrel for measurement and reaction.

* Alumina with grease bonding.

- Hard to machine

- Thermal contraction less than $\mathrm{Nb}_{3} \mathrm{Sn}$ wire.

- Could use same mandrel for measurement and reaction. (differential expansion?) 

APPENDIX N

GUIDELINE FOR $\mathrm{Nb}_{3} \mathrm{Sn}$ CRITICAL CURRENT MEASUREMENTS USING FIBERGLASS-EPOXY COMPOSITE SAMPLE MANDRELS

L. F. Goodrich

Prepared for: Office of the VAMAS Technical Working Area 6 Superconducting and Cryogenic Structural Materials 
Guideline for $\mathrm{Nb}_{3} \mathrm{Sn}$ Critical Current Measurements Using Fiberglass-Epoxy Composite Sample Mandrels

L. F. Goodrich

Superconductor and Magnetic Measurements Group

National Institute of Standards and Technology

Boulder, CO 80303

\section{PREFACE}

This guideline was prompted, in part, by presentations given at the 6 th Japan-U.S. Workshop on High Field Superconductors (February 22-24, 1989 Boulder, Colorado) and subsequent discussions. The results of the recent Versailles Project on Advanced Materials and Standards (VAMAS) $\mathrm{Nb}_{3} \mathrm{Sn}$ criticalcurrent round robin indicate that increased consistency in interlaboratory measurements might be achieved by a more detailed specification of the critical-current $\left(I_{C}\right)$ measurement technique. However, a competition exists between the benefit of a rigidly specified measurement technique, which ensures measurement reproducibility, and a less restrictive technique that would be practical for a greater number of laboratories. Ideally, the measurement technique should have the least number of restrictions that is consistent with measurement reproducibility.

This measurement guideline is intended to be one of a few different measurement techniques in a second $\mathrm{Nb}_{3} \mathrm{Sn} \mathrm{I}_{c}$ round robin that is envisioned to determine the preferred measurement method for $\mathrm{Nb}_{3} \mathrm{Sn}$. Each participating laboratory, in this second round robin, will make measurements using each technique. The number of laboratories participating in this second round robin might be considerably reduced, as compared with the first round robin, because of the difficulty involved in complying with the necessarily restrictive specifications for the various measurement techniques. However, in the event that the second round robin results in a general consensus regarding which measurement variables must be specified in order to assure consistent measurements among different laboratories, a third round robin might be useful for determining the effectiveness of the measurement method for a larger group of laboratories.

At this time, there is no consensus regarding the materials used for the reaction mandrel, measurement mandrel, or sample bonding. There are advantages and disadvantages for all known materials. This guideline was written for one combination of materials. It may not be the best, but it represents one feasible option. The materials used in this technique are stainless steel tubes for reaction mandrels, G-10CR cylinders cut from plate stock for measurement mandrels, and silicone based vacuum grease as the bonding material.

This measurement guideline assumes that the reader has the basic knowledge and experience with $\mathrm{I}_{\mathrm{C}}$ measurements of $\mathrm{NbTi}$ and $\mathrm{Nb}_{3} \mathrm{Sn}$ conductors. If not, the basic information on these measurements can be found elsewhere [1, 2 , and 3]. 


\section{INTRODUCTION}

The results of the VAMAS $I_{c}$ round robin demonstrated the existing measurement discrepancy among a large number of laboratories for several different $\mathrm{Nb}_{3} \mathrm{Sn}$ samples [4]. The discrepancy was significant. A major source of this discrepancy was determined to be varying degrees of axial sample strain caused by variations in the thermal contraction of different sample mandrels [5].

The sample bonding technique can play an important role in this effect because the strength of the bonding determines the amount of the sample mandrel's thermal contraction that is transmitted to the sample. A relatively weak bond can inhibit this transmission of thermal contraction but it may also allow sample motion under the influence of the Lorentz force, which, in turn, can cause variations in the measured $I_{C}$. A high strength bonding material used in conjunction with a sample mandrel whose thermal contraction is comparable to that of the $\mathrm{Nb}_{3} \mathrm{Sn}$ sample alleviates both of these mechanical problems but it introduces a practical problem. The commonly used sample mandrel material that has satisfactory thermal contraction is a fiberglassepoxy composite (G-10). The high strength bonding material that is typically used for G-10 sample mandrels is a filled epoxy adhesive. The problem with this method is that the epoxy forms a fairly permanent bond which makes repeated use of the sample mandrel difficult. Clearly what is needed is a bonding material that provides adequate sample support but that is easily removable from the sample mandrel. The results of the VAMAS measurements indicate that grease may be adequate for this purpose as long as all of the $I_{C}$ measurements are made with the Lorentz force directed inward, toward the central axis of the sample mandrel.

Grease is a generic term that applies to a very broad range of heavy lubricants that have significantly different physical properties. Two such materials that have been used for sample bonding materials are petroleum jelly and silicone-based vacuum grease. In this document, grease means siliconebased vacuum grease.

Recent research at NIST indicates that a G-10 cylinder appropriately machined from plate stock has a thermal contraction that does not vary significantly with its geometry and is close to that of a $\mathrm{Nb}_{3} \mathrm{Sn}$ wire sample. Earlier research indicated that the thermal contraction of rolled fiberglassepoxy composite tubes varies significantly with the wall thickness of the tube [5]. Because these composites are anisotropic, the orientation of the material's internal structure, relative to the tube, is important. Figure 1 illustrates several fiberglass-epoxy composite geometries. A plate tube can be made by machining a cylindrical tube from thick fiberglass-epoxy plate stock with the axis of the tube perpendicular to the surface of the plate. There is a commercially available rod that is machined from plate stock, but the axis of this rod is in the plane of the plate. A rod of this type will have a very anisotropic radial thermal contraction which is unacceptable for this application.

A plate tube can be machined to make a sample measurement mandrel. The inner and outer diameters of the plate tube can be machined to receive current 
contacts and to be mounted on the test fixture without significantly affecting the thermal contraction of the G-10. There is an advantage to having extra wall thickness however; it reduces the chance of delamination. The thickness of the plate stock will determine the maximum overall length of the plate tube. It is better to start with a single plate than to build up the thickness by gluing multiple plates together. The plate tube can be machined from a plate using a hole saw or by cutting out a parallelepiped then turning it on a lathe. A hole saw is a tool that can be used to drill large diameter holes. It can also be used to extract a cylindrical section from a plate, which can be turned on a lathe to the final diameter. Water is commonly used as a machining lubricant for G-10; it also reduces the dust and keeps the temperature of the G-10 low.

\section{MATERIALS}

The materials used in this technique are stainless steel tubes for reaction mandrels, G-10 cylinders cut from plate stock for measurement mandrels (plate tubes), and silicone-based vacuum grease as the bonding material. The stainless steel tubes and silicone-based vacuum grease are readily available and have consistent properties for this application. The G-10 plate, especially plate of the necessary thickness, is not as available and the consistency of its properties is not known. Thus, a single lot of G10 should be acquired. Dr. Hitoshi Wada of Japan's National Research Institute for Metals has expressed a willingness to purchase a common lot of this material, thus, minimizing variations in properties.

\section{A G-10, NEMA (National Electrical Manufacturers' Association)} designation, measurement mandrel material was selected because it can be oriented to have a thermal contraction that does not vary significantly for various geometries and it is close to that of a $\mathrm{Nb}_{3} \mathrm{Sn}$ wire sample. Cryogenicradiation ( $C R$ ) grade material should be purchased, if possible, since the manufacturing specifications for $C R$ grade materials are more stringent and their performance at cryogenic temperatures is more predictable. The use of $\mathrm{CR}$ grade material would also allow for better reproducibility in the future.

Thick G-10CR plate is available from a number of sources in the U.S. and a sheet or two may be sufficient to supply the participants with material. A full sheet is about 91 by $122 \mathrm{~cm}$ ( 3 by 4 feet) and a $5 \mathrm{~cm}$ thickness would cost about $\$ 2,000$ plus freight $(110 \mathrm{~kg})$. A full sheet may be enough material for 400 sample mandrels. The key parameter is the thickness of the plate since this will determine the maximum length of the measurement coil. The measurement mandrel needs to be machined from the plate stock with the cylinder axis normal to the plate surface in order to better match the thermal contraction of the $\mathrm{Nb}_{3} \mathrm{Sn}$ coiled sample. The thickness of the plate should be at least $5 \mathrm{~cm}$. The plate stock could be cut into strips that are slightly wider than the measurement mandrel diameter for distribution to participants of this round robin. This would facilitate machining the cylinders, which is desirable since G-10CR is somewhat difficult to machine. Each participant should be supplied with enough material to construct several (5 to 10) mandrels to allow for different wire diameters and mistakes during machining. 
PROCEDURE

General Philosophy

Because this measurement technique will be conducted in many laboratories, it is impractical to specify all of the details of the sample reaction, mounting, and measurement procedures. For example, a detailed specification might require that the sample mandrels have an outside diameter that is slightly different than that normally used at the testing laboratory, and compliance with this somewhat arbitrary specification would unnecessarily increase the required time and cost for this study. Consequently, the philosophy of this guideline is to be as general as is practical and, thus, allow the measurement laboratories to adhere to their normal practices as much as possible.

Please read through all of these procedures before implementing this guideline. It was not written as a step-by-step procedure. Some elements are described out-of-sequence because of the inter-relationship between steps and various options that are presented.

Sample Reaction Mandrel

The samples should be reacted on well oxidized stainless steel tubes in order to avoid diffusion bonding between the sample and the tube. When diffusion bonding occurs, the probability of damaging the sample when transferring it to the measurement mandrel is greatly increased. Even when diffusion bonding does not occur, extreme care must be exercised in transferring the sample to avoid damage due to bending strain. The reaction mandrel should have a helical groove for defining the sample coil's pitch. The sample should be well seated in this groove, but not tight, prior to reaction to insure reproducibility of the coil diameter and pitch. The wire can be retained on the reaction mandrel by bending the wire through a hole at each end of the mandrel. Internal-tin processed conductors need to have all wire ends well outside the reaction furnace, unless the operator is specially trained to weld the wire ends.

Sample Measurement Mandrel

The G-10 measurement mandrel should have the same pitch length, groove geometry, and a diameter that is approximately $0.5 \%$ larger than the stainless steel reaction mandrel to insure that the reacted sample can be fit to the measurement mandrel with a minimum of sample deformation. The samples should be well seated in the measurement mandrel's groove to ensure consistent coil diameter and, thus, minimize bending strain. This is also important for minimizing the thickness of the bonding material (grease) between the sample and mandrel. Moreover, a thick layer of grease is more likely to yield under the influence of the Lorentz force than a thin layer, where the sample is substantially in contact with the G-10 mandrel.

A good choice for the mandrel's groove geometry is a $60^{\circ}$ "V" with a fairly sharp point. The $60^{\circ}$ groove provides good sample support in both the radial and transverse directions. The sharp point of the groove insures that 
the conductor contacts the groove at the points of tangency rather than at the bottom of the groove. In the case of the measurement mandrel, this should minimize the thickness of the bonding-material layer between the sample and the mandrel. In the case of the reaction mandrel, it may reduce diffusion bonding. The groove should be deep enough that the sample contacts the mandrel within the groove rather than at the interface formed between the groove and the mandrel's surface. This is important for transverse stability of the sample. One detrimental consequence that results from making the groove too deep is that the sample will require greater deformation during the transfer process in order to clear the mandrel.

Sample Mounting

Before attempting to transfer the sample from the reaction mandrel, check it for diffusion bonding to the mandrel. If there is significant bonding that might result in sample damage, the sample should be discarded. Two methods can be used for removing the sample from the reaction mandrel: The first requires a very smooth interface between the sample and mandrel but, when it can be employed, allows sample removal with minimal deformation. In this method the sample is unthreaded from the mandrel by lightly gripping the sample coil's surface while turning the mandrel. Alternatively, the sample coil's diameter can be expanded by carefully untwisting the coil slightly until the mandrel can be extracted. Although this second technique could result in some sample deformation, it may be the better method depending on groove depth, the number of turns, and the operator.

It is necessary to have a complete, but thin, coat of grease under the sample on the grooved G-10 mandrel. This is difficult or impossible to maintain if the sample is threaded onto the measurement mandrel. However, the sample could be threaded completely onto the measurement mandrel, then expanded slightly and moved just out of the groove to a position between the grooves on the mandrel. With the sample in this position, the groove could be filled with grease and then the sample could be slipped back into the groove. In the other case, where the coil is expanded to clear the mandrel, the grease should be applied to the sample groove prior to sample transfer.

Voltage tap wires can be attached to the sample after it is seated in the groove. There is a chance that the grease will interfere with the soldering operation or that the elevated temperature will affect the properties of the grease, but these are not expected to be significant. If the voltage tap wires are attached to the sample before the sample is set into the greased groove, there are two possible options depending upon how the sample is transferred from reaction to measurement mandrel. If the sample is threaded onto the measurement mandrel, the voltage tap wires could be attached to the sample after it has been transferred to the measurement mandrel but before it has been set in its groove. In the case where the sample is transferred directly to a greased measurement mandrel, the voltage tap wires could be soldered to the sample while it is on the reaction mandrel. In all cases, the single voltage tap wires can be counter wound onto the coil and pairs of wires twisted together after the sample is attached to the current contacts. 
The amount of grease that is necessary will vary depending upon the details of the groove geometry and sample diameter. In most cases it is expected that a thin coat on the surfaces of the groove would be a sufficient amount of grease. A little extra grease could be applied to reduce the chance of an incomplete coat. Too much extra grease in the groove will make it more difficult to keep the grease layer thin, since the excess grease will act like a hydraulic fluid and require excess pressure to extrude past the sample. A thick layer of grease might lead to a less rigid support for the sample under the influence of the Lorentz force. Any excess grease outside the groove should be removed after the sample is in place so that sample cooling is not inhibited. In cases where sample motion due to mechanical vibration is expected, grease could be added to fill the sides of the groove after the sample is seated. Since all of the measurements will be made with the Lorentz force directed inward, there is limited benefit in having excess grease on top of the sample.

Before soldering the sample to both of its current contacts, remove the slack in the sample coil by firmly seating it in the sample groove. This is easily achieved by first soldering one end of the sample coil to its current contact and then, starting at that contact, pressing the sample into its groove in a continuous motion that results in all of the coil's slack being transferred to the opposite current contact. The sample can then be fixed in this taut position by soldering it to the current contact. Neither current contact should have more than 0.9 of a turn soldered to the current contact. If more than one turn is soldered to the current contact there is a chance that this loop will carry a slowly decaying current (induced by a change in the background field), which will affect the magnetic field of the sample.

\section{Sample Cooling Rate}

Different laboratories probably cool their samples at significantly different rates; in the limiting cases, some may slowly cool the sample in helium vapor while others may insert the sample directly in a liquid-helium bath (or perhaps liquid nitrogen). This variable may effect the measured $I_{C}$. The grease bonding material has virtually no mechanical strength until it is cooled below its freezing temperature. Consequently, the strength of the coupling between the sample and its mandrel is changing during the cooling process while differential thermal contraction between the sample and mandrel is occurring. This fact may result in different mechanical states for samples cooled at different rates. This guideline suggest that the sample should be cooled from room temperature to liquid helium (or liquid nitrogen) temperature over a time period of at least 5 minutes.

\section{Current Reversal}

Grease will undoubtedly present a limitation in the $I_{c}$ measurements that other more permanent bonding materials do not. Its relatively low tensile strength will probably disallow its effective use for $I_{c}$ measurements where the Lorentz force on the sample coil is directed outward. This, in turn, disallows sample-current reversal for testing the effect of the sample coil's self-generated magnetic field on the measured $I_{C}$. 
Field History and Repeat Determinations

Because the Lorentz force may play an important role in the $I_{c}$ measurements of grease-mounted samples, the magnetic field history of the test sample may affect the measurements. For example, if the first $I_{c}$ measurement is made at the field where the Lorentz force on the sample is a maximum, the subsequent measurements might be different than for another field sequence. Consequently, the measurement sequence should be carefully considered and recorded. It is recommended that the first measurement be at the field closest to where the Lorentz force is maximum (the force peaks at about $5 \mathrm{~T}$ for $\mathrm{Nb}_{3} \mathrm{Sn}$ ). Also, if the Lorentz force causes irreversible sample motion, subsequent measurements at the same magnetic field may have different results. Repeat determinations of the $I_{c}$ should be used to detect mechanical deterioration of the sample bond. Repeat determinations are suggested for this guideline, especially for the first magnetic field at the peak Lorentz force.

\section{DISCUSSION}

Diffusion bonding between the sample and the reaction mandrel has been a significant problem. In addition to oxidization of the surface, baking (prior to winding a $\mathrm{Nb}_{3} \mathrm{Sn}$ sample) a sprayed graphite lubricant into stainless steel reaction mandrels has been suggested and we found it beneficial. With use, the surface of the stainless steel becomes more passive. Other reaction mandrel materials such as graphite and alumina have been suggested. A related issue that has been mentioned, is that the thermal expansion of the reaction mandrel material will affect the ultimate $I_{c}$ of the wire that is reacted on it. The postulate is that the wire irreversibly shortens during the initial anneal of the reaction heat treatment and then the wire is stretched more by mandrels that expand more, resulting in a lower $I_{c}$. This would suggest that reaction mandrel materials such as graphite or alumina would be better than stainless steel.

There are some unresolved issues regarding sample motion and support on the measurement mandrel. The grease bonding material may not be necessary and, in fact, it may cause some adverse effects. Some possible effects were mentioned above, such as; grease may interfere with the soldering operation, it may inhibit seating the sample into the groove, and it might inhibit sample cooling. If the thermal contraction of the mandrel is less than the sample from room to liquid-helium temperature, then the sample will tighten onto the mandrel upon cooling and a bonding material may not be necessary except in cases were mechanical vibration is expected. It is also possible that grease will act like a lubricant and enhance conductor motion. Another related consideration is the angle of the groove. It is possible that a $90^{\circ}$ groove angle would be better than $60^{\circ}$ because the sample will seat more firmly. A $60^{\circ}$ angle was selected for this guideline because it is more commonly used to machine grooves.

There have been discussions about the feasibility of a standard coil diameter and pitch. There are serious consequences to the selection of a coil diameter. If the coil diameter is selected to allow measurements in the smallest magnet bore that is used for $I_{c}$ measurements, then probability of 
sample damage due to handling increases. Alternatively, if a larger coil diameter is selected, a number of laboratories may not be able to participate. There are also significant benefits to standardizing coil diameter and pitch. This may be the most effective way to achieve consistent $I_{c}$ measurements. Batch fabrication of reaction and measurement mandrels with common materials could be cost effective and allow more interchangeable combinations of reaction and measurement mandrels. This could also facilitate the exchange of samples among laboratories.

With standardization of coil diameter and pitch, there are a number of possibilities that might become cost effective. Alumina reaction mandrels is a good example. Another option is reaction mandrels made by machining a groove into a stainless steel tube, then spraying a coat of alumina (or some other ceramic) onto the tube. This would be a lower cost option than machining an alumina-tube reaction mandrel, and it would not present the problem of diffusion bonding that an oxidized stainless steel mandrel does. Another option that standardization creates is the use of rolled G-10 tubes for measurement mandrels. A low wall-to-radius ratio could be selected to give a thermal contraction that is well matched to the thermal contraction of $\mathrm{Nb}_{3} \mathrm{Sn}$. Rolled tubes would be much easier to fabricate than plate tubes. A lot of material could be purchased and, thus, the variability of the thermal contraction should be negligible. A rolled-tube measurement mandrel might be appropriate for this test, since we are only trying to find a consensus method that gives consistent results.

At this time, stainless steel seems to be the more common reaction mandrel material, so this guideline is based on it. It is expected that the measurement mandrel material and bonding method will be the more dominant factors and will be tested regardless of reaction mandrel material.

\section{REFERENCES}

1. L. F. Goodrich and F. R. Fickett, "Critical current measurements: a compendium of experimental results," Cryogenics 22, 225-241 (1982).

2. Standard Definitions of Terms Relating to Superconductors, Annual Book of ASTM Standards, ASTM B713-82, Part 2.03, pp. 591-594, American Society for Testing and Materials, Philadelphia, PA (1983).

3. Standard Test Method for D-C Critical Current of Composite Superconductors, Annual Book of ASTM Standards, ASTM B714-82, Part 2.03, pp. 595-598, American Society for Testing and Materials, Philadelphia, PA (1983).

4. K. Tachikawa, K. Itoh, H. Wada, D. Gould, H. Jones, C. R. Walters, L. F. Goodrich, J. W. Ekin, and S. L. Bray, "VAMAS intercomparison of critical current measurement in $\mathrm{Nb}_{3} \mathrm{Sn}$ wires," IEEE Trans. on Magn. 25(2) 2368-2374 (March 1989).

5. L. F. Goodrich, S. L. Bray, and T. C. Stauffer, "Nb3 3 Sn critical-current measurements using tubular fiberglass-epoxy mandrels," IEEE Trans. on Magn. 25 (2) 2375-2378 (March 1989). 


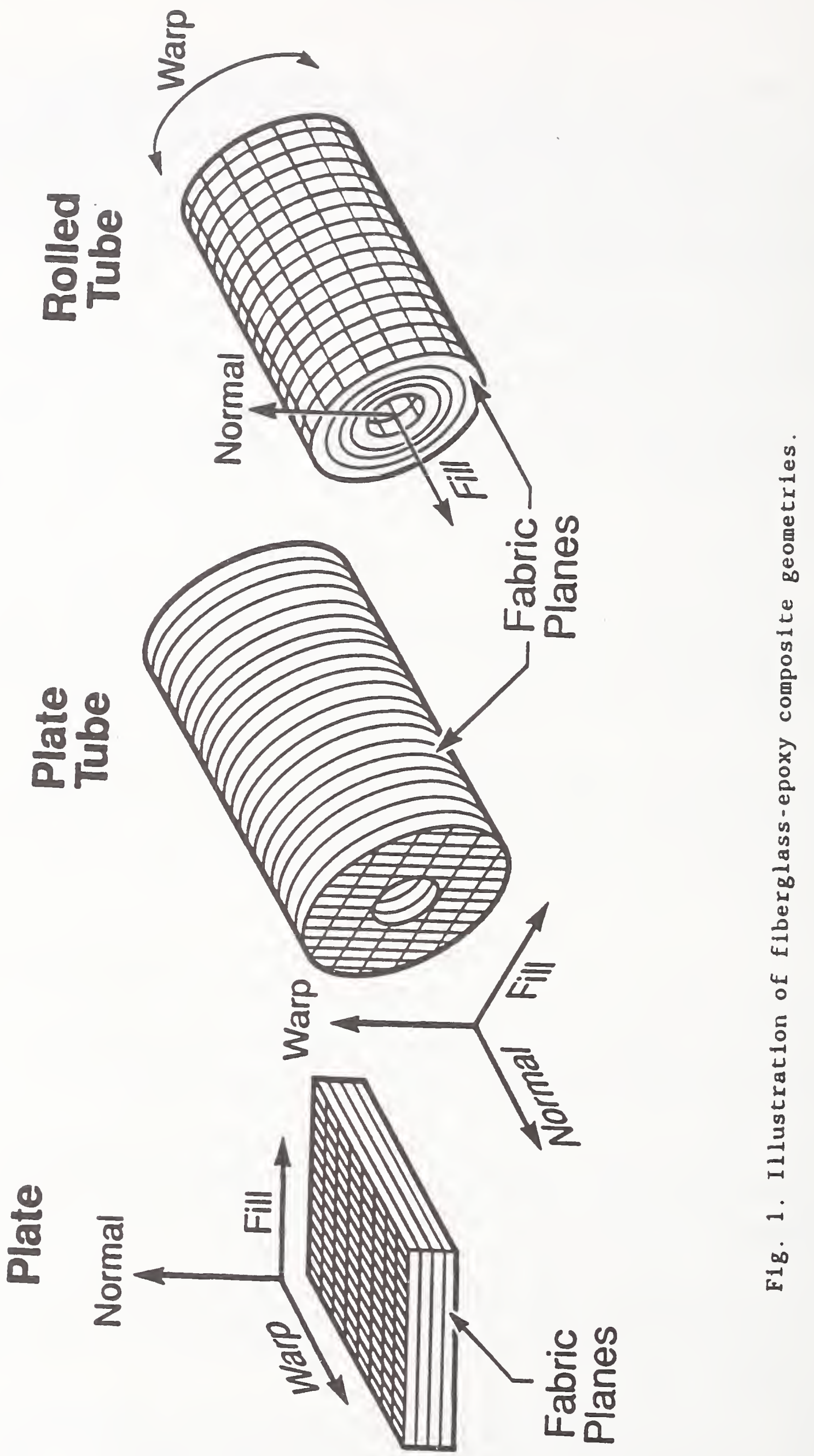




\section{BIBLIOGRAPHIC DATA SHEET}

1. PUBLICATION OR REPORT NUMBER

2. PERFORMING ORGANIZATION REPORT NUMBER

3. PUBLICATION DATE

January 1990

\section{TITLE AND SUBTITLE}

DEVELOPMENT OF STANDARDS FOR SUPERCONDUCTORS

Final Report

January 1988-December 1989

5. AUTHOR(S)

L.F. Goodrich, R.B. Goldfarb, and S.L. Bray

6. PERFORMING ORGANIZATION (IF JOINT OR OTHER THAN NIST, SEE INSTRUCTIONS)

U.S. DEPARTMENT OF COMMERCE

NATIONAL INSTITUTE OF STANDARDS AND TECHNOLOGY

NATIONALINSTITUTE OF STANDARDS AND TECHNOLOGY 80303-3328

7. CONTRACT/GRANT NUMBER

8. TYPE OF REPORT AND PERIOD COVERED

9. SPONSORING ORGANIZATION NAME AND COMPLETE ADDRESS (STREET, CITY, STATE, ZIP)

Office of Fusion Energy and Division of High Energy Physics

Department of Energy

Washington, DC 20545

10. SUPPLEMENTARY NOTES

DOCUMENT DESCRIBES A COMPUTER PROGRAM; SF-185, FIPS SOFTWARE SUMMARY, IS ATTACHED.

11. ABSTRACT (A 200-WORD OR LESS FACTUAL SUMMARY OF MOST SIGNIFICANT INFORMATION. IF DOCUMENT INCLUDES A SIGNIFICANT BIBLIOGRAPHY OR LITERATURE SURVEY, MENTION IT HERE.)

A cooperative program with the Department of Energy, the National Institute of Standards and Technology, other national laboratories, and private industry is in progress to develop standard measurement practices for use in large-scale applications of superconductivity. This report describes research for the period January 1988 through December 1989. It contains the results of critical current studies on a large conductor Reference Material (RM), the effect of power-supply current ripple, the measurements of large conductors, and an interlaboratory comparison (round robin) of $\mathrm{Nb}_{3} \mathrm{Sn}$ wires. Short-range variations in magnetic hysteresis loss in multifilamentary $\mathrm{Nb}_{3} \mathrm{Sn}^{3}$ were studied. The results of participation in several interlaboratory comparison are described.

12. KEY WORDS (6 TO 12 ENTRIES; ALPHABETICAL ORDER; CAPITALIZE ONLY PROPER NAMES; AND SEPARATE KEY WORDS BY SEMICOLONS) ac losses; circuit diagrams; critical current; current ripple; hysteresis; magnetization; niobium-tin; niobium-titanium; power supply; round robin; standards; superconductors; susceptibility

\section{AVAILABILITY}

 UNLIMITED FOR OFFICIAL DISTRIBUTION. DO NOT RELEASE TO NATIONAL TECHNICAL INFORMATION SERVICE (NTIS). ORDER FROM SUPERINTENDENT OF DOCUMENTS, U.S. GOVERNMENT PRINTING OFFICE, WASHINGTON, DC 20402.

ORDER FROM NATIONAL TECHNICAL INFORMATION SERVICE (NTIS), SPRINGFIELD, VA 22161.

14. NUMBER OF PRINTED PAGES 148 


